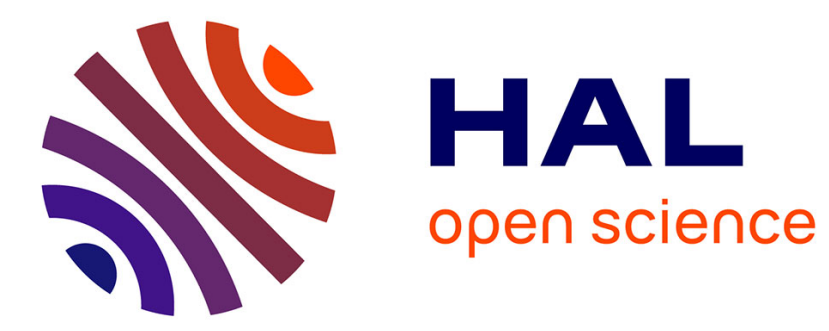

\title{
PEGASES: Plasma Propulsion with Electronegative Gases
}

Gary Leray

\section{To cite this version:}

Gary Leray. PEGASES: Plasma Propulsion with Electronegative Gases. Physics [physics]. Ecole Polytechnique X, 2009. English. NNT : 2009EPXX0066 . pastel-00005935

\section{HAL Id: pastel-00005935}

\section{https://pastel.archives-ouvertes.fr/pastel-00005935}

Submitted on 20 May 2010

HAL is a multi-disciplinary open access archive for the deposit and dissemination of scientific research documents, whether they are published or not. The documents may come from teaching and research institutions in France or abroad, or from public or private research centers.
L'archive ouverte pluridisciplinaire HAL, est destinée au dépôt et à la diffusion de documents scientifiques de niveau recherche, publiés ou non, émanant des établissements d'enseignement et de recherche français ou étrangers, des laboratoires publics ou privés. 


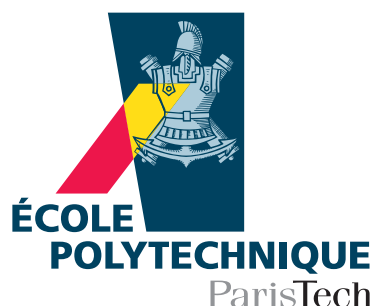

Thèse présentée pour obtenir le grade de

DOCTEUR DE L'ÉCOLE POLYTECHNIQUE

Spécialité:

Physique des Plasmas

Présentée par:

Gary Leray

\section{PEGASES: Plasma Propulsion with Electronegative Gases}

Thèse soutenue le 15 octobre 2009

Jury composé de:

Gerjan Hagelaar, Rapporteur

Khaled Hassouni, Rapporteur

Pascal Chabert, Directeur

Gilles Cartry

Stéphane Mazouffre

Bill Graham

Pere Roca i Cabarrocas, Président
IR CNRS - LAPLACE, U Toulouse III

Professeur - LIMHP, U Paris XIII

DR CNRS - LPP, Ecole Polytechnique

MC - PIIM, U Provence

CR - ICARE, U d'Orléans

Professeur - CPP, Queen's University Belfast

DR CNRS - LPICM, Ecole Polytechnique 



\section{Acknowledgements}

First and foremost, I would like to thank Pascal Chabert for the opportunity he gave me to work on a $\mathrm{PhD}$ thesis with a brand new concept of plasma thruster. This allowed me to learn different aspects of plasma physics: from the design of an entire plasma system and probes, through theoretical and computational modeling, to experiments with multiple plasma reactors and probes.

I would also like to thank professors Allan Lichtenberg and Michael Lieberman for welcoming me on several occasions to Cory Hall in UC Berkeley, California. The collaboration on this project has been invaluable and the experience enlightening. Moreover, I would like to thank Allan and Elizabeth Lichtenberg for often inviting me to their home and for excursions in the Bay Area.

I would like to thank the members of my jury: Gerjan Hagelaar and Khaled Hassouni, my examiners, who allowed me to defend; Gilles Cartry, Stephane Mazouffe, Bill Graham and Pere Roca i Cabarrocas, who reviewed my manuscript and defense.

I would like to thank Jean-Luc Raimbault for the multiple discussions we had on plasma modeling. I learned a lot from his experience and insight. Jean-Paul Booth for his experimental knowledge, and Jean Larour for his knowledge in radio-frequency interferences.

I would like to warmly thank Jean Guillon for his help designing plasma setups and probes. His experience in mechanical design for vacuum systems proved invaluable, as well as plasma reactor design. Also, all the members of the electronic and work shops in the lab: Bruno D, Jean-Paul S, Christian K and Mickael B. A big thank to the lab staff: Cathy P, Cherifa Z, Isabelle L, Philippe A.

Of course, I would like to thank all the other PhD Students/Post-Docs in the lab. Special mention to Emilie and Laurent: I highly enjoyed being part of the PRAGM trio, from working, through breaks and discussions, to fun out of the office. Also Paul, who was my lab partner during the Master degree, for discussions and fun. As to the others, a list in alphabetical order is only fair: Albert, Ane, Binjie, Claudia, Cormac, Cyprien, Daniil, Dragana, Elisée, Garrett, Jaime, Joseph, Katia, Lara, Lina, Lorenzo, Nico B, Nico P, Olivier, Pierre, Richard, Seb C, Seb D, Sedina, Xavier.

I would like to thank Gilles, my brother, and Sophie and Jean-Luc, for their friendship and time. Also Sarah and Romain for their friendship. Special mention to Frantisek for all the fun we have had all these years. 
A huge thanks to my mother, for all her help and support, especially during chaotic life changes during my college years.

Finally, Liz. My stay in Berkeley feels like it turned form gray to full color thanks to you, even though I was having fun to begin with. Thank you for all your support and help, and thank you for proof-reading my manuscript (physics looks so evil to an English major...).

Thank you for everything. 


\section{Contents}

Acknowledgements $\quad$ iii

Symbols and Abbreviations $\quad$ ix

1 Introduction $\quad 1$

1.1 Space Propulsion . . . . . . . . . . . . . . . . . . . . . 1

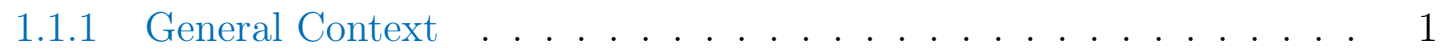

1.1 .2 Rocket Versus Electrical . . . . . . . . . . . . . . . . . 3

1.1.3 Electrical Propulsion . . . . . . . . . . . . . . . . . . 6

1.1 .4 Newer Concepts . . . . . . . . . . . . . . . . . . 9

1.2 PEGASES: Plasma Propulsion with Electronegative GASES . . . . . . . . 10

1.2 .1 The Idea . . . . . . . . . . . . . . . . . . . . . 10

1.2.2 Advantages of PEGASES . . . . . . . . . . . . . . . . . . . 13

1.2 .3 Timeline and Financing . . . . . . . . . . . . . . 13

1.3 Outline . . . . . . . . . . . . . . . . . . . . 14

2 Models and Diagnostics for Plasmas $\quad 17$

2.1 Models for Plasmas . . . . . . . . . . . . . . . . . . . . . 17

2.1 Notations and Units . . . . . . . . . . . . . . . . . . . . 17

2.1.2 The Various Models . . . . . . . . . . . . . . . . . . . 18

2.1.3 Maxwellian Species . . . . . . . . . . . . . . . . . . 22

2.1.4 Boltzmann Relation . . . . . . . . . . . . . . . . . . 22

2.2 Diagnostics . . . . . . . . . . . . . . . . . . . 22

2.2.1 Langmuir Probes . . . . . . . . . . . . . . . . . . . . 23

2.2.2 Voltage and Current Probe. . . . . . . . . . . . . . . 29

2.2.3 Retarding Field Energy Analyzer . . . . . . . . . . . . . . . . . . 29

2.2 .4 Instruments . . . . . . . . . . . . . . . . . . . . . . . . . . . . . . . . . . . .

2.2.5 Computer Programs . . . . . . . . . . . . . . . 33

2.3 Electronegativity Measurement Techniques . . . . . . . . . . . . . . . 34

2.3.1 Review of Techniques . . . . . . . . . . . . . . 34

2.3.2 Electrostatic Probe . . . . . . . . . . . . . . . 36

$\begin{array}{lll}\text { I } & \text { Experiments } & 39\end{array}$

3 Experimental Setups $\quad 41$

3.1 Helicon reactor . . . . . . . . . . . . . . . . . . . . . 41

3.2 PEGASES, Prototype I . . . . . . . . . . . . . . . . . . . . . 43

3.2 .1 Geometry .......................... 43 
3.2 .2 Source Region . . . . . . . . . . . . . . . . . . . 46

3.2 .3 Additional Magnets . . . . . . . . . . . . . . . . . . . . . . 47

3.3 Thruster Chamber and Matchboxes . . . . . . . . . . . . . . . . . . . 47

3.3.1 Thruster Chamber . . . . . . . . . . . . . . . 47

3.3 .2 Matchboxes ......................... 51

4 Ionization Stage $\quad 53$

4.1 Power Coupling, Symmetry and Stability . . . . . . . . . . . . . . . . 54

4.1 .1 Helicon Mode . . . . . . . . . . . . . . . . . . 54

4.1 .2 Design Evolution . . . . . . . . . . . . . . . . . . 55

4.1 .3 Plasma Asymmetry . . . . . . . . . . . . . . . . . . . . . . . . . . . . . . . . 60

4.1 .4 Instabilities . . . . . . . . . . . . . . . 63

4.2 Positive Ion Current Density . . . . . . . . . . . . . . . . . . . . . . . . . . . . . . 65

4.2 .1 Original Matchbox Circuit . . . . . . . . . . . . . . 66

4.2 .2 Modified Matchbox Circuit . . . . . . . . . . . . . . 67

4.3 Mass Efficiency . . . . . . . . . . . . . . . . . . . . 70

4.3 .1 Definition .............................. 70

4.3.2 Non-Uniformity of the Current Density . . . . . . . . . . . . 71

4.3 .3 Results. . . . . . . . . . . . . . . . . . . . . . . . . . . . . . 72

4.4 Electron Temperature . . . . . . . . . . . . . . . . . . . . . . . . . . . . . . .

4.4 .1 Interest . . . . . . . . . . . . . . . . . . . . . . . . . . . . . . . . 73

4.4 .2 Discussion of the Results . . . . . . . . . . . . . . . . . 74

4.5 Conclusion . . . . . . . . . . . . . . . . . . . . . . . . . . . . . . . . . . .

4.5.1 Power Coupling . . . . . . . . . . . . . . . . . 77

4.5.2 Positive Ion Current Density . . . . . . . . . . . . . . . . . 78

4.5 .3 Mass Efficiency . . . . . . . . . . . . . . . . . . . 78

4.5.4 Electron Temperature . . . . . . . . . . . . 78

5 Magnetic Electron Filtering Stage - Experiments $\quad 81$

5.1 Experimental Background on Ion-Ion Plasmas . . . . . . . . . . . . . . . 82

5.1 .1 Ion-Ion Plasma Creation . . . . . . . . . . . . . . . 82

5.1 .2 Electronegative Plasma Afterglow . . . . . . . . . . . . . . 82

5.1 .3 Magnetic Electron Filtering . . . . . . . . . . . . . . 83

5.2 Helicon Reactor . . . . . . . . . . . . . . . . . . . 83

5.2 .1 Electron Density and Positive Ion Flux . . . . . . . . . . . . 83

$5.2 .2 \mathrm{I}-\mathrm{V}$ Characteristics . . . . . . . . . . . . . . . 86

5.2.3 Plasma and Floating Potentials, Electron Temperature . . . . . . . 89

5.2 .4 RFEA Measurements . . . . . . . . . . . . . . . . . . . . . . 92

5.2 .5 Electronegativity Measurements . . . . . . . . . . . . . . . . 95

5.3 PEGASES Prototype I . . . . . . . . . . . . . . . . . . . 96

5.3 .1 I-V Characteristics . . . . . . . . . . . . . . . . . . . . . . . . . . . . . 97

5.3 .2 Electronegativity Measurements . . . . . . . . . . . . . . . . . . 97

5.4 Design Improvements . . . . . . . . . . . . . . . . . . . . 100

5.4.1 Enhanced Magnetic Filtering . . . . . . . . . . . . . . 100

5.4 .2 Optimized Neutral Injection . . . . . . . . . . . . . . . . . . . . . . . . . . . . . . . . . . . . . . . . .

5.5 Conclusions . . . . . . . . . . . . . . . . . 103 
II Models

6 Magnetic Electron Filtering Stage - Theory 107

6.1 Franklin Fluid Model in One Dimension . . . . . . . . . . . . . . . . . 107

6.2 Fluid Model Including End Losses . . . . . . . . . . . . . . . . . . . . . . 110

6.2.1 Limitations of a 1D System . . . . . . . . . . . . . . . . 110

6.2.2 Electron End Loss . . . . . . . . . . . . . . . . . . . 111

6.2.3 Reformulated Model with End Loss . . . . . . . . . . . . . . . . 113

6.2.4 Results Using the Reformulated Model . . . . . . . . . . . . . . . . . 116

6.3 Analytic Approximation . . . . . . . . . . . . . . . . . . 124

6.3.1 Analytic Model . . . . . . . . . . . . . . . . . . . 124

6.3.2 Comparison with Numerics, Parameter Scaling . . . . . . . . . . . 130

6.4 Model Limitations . . . . . . . . . . . . . . . . . . . . . 133

6.4.1 Symmetrical Ion-Ion Plasma . . . . . . . . . . . . . . . . 133

6.4.2 Gas Electronegativity . . . . . . . . . . . . . . . 134

6.4.3 Solutions with $n_{e}(R) \neq 0 \ldots \ldots . \ldots \ldots 135$

6.4 Constant $T_{e} \ldots \ldots \ldots \ldots \ldots \ldots \ldots$

6.4.5 Non-Uniform Neutral Density . . . . . . . . . . . . . 136

7 Ion Extraction Stage $\quad 137$

7.1 Description of the Problem . . . . . . . . . . . . . . . 138

7.1 .1 High Voltage Sheath . . . . . . . . . . . . . . . . . . 138

7.1 .2 Low Voltage Sheath . . . . . . . . . . . . . . . . . . . . . 139

7.2 Basic Fluid Model Study . . . . . . . . . . . . . . . . . . . . . 139

7.2.1 Motivation. . . . . . . . . . . . . . . . 139

7.2 .2 Assumptions . . . . . . . . . . . . . . . . . . . 140

7.2 .3 Poisson and Model Equations . . . . . . . . . . . . . . . . . . 141

7.2 .4 Ion-Ion Plasma . . . . . . . . . . . . . . . . . . . 147

7.3 PIC Simulations . . . . . . . . . . . . . . . . . . . . . 147

7.3.1 Description of the PIC Simulations . . . . . . . . . . . . . 147

7.3 .2 Results . . . . . . . . . . . . . . . . . . . . . . . . . . . . . . . . . . . . . . . . . . .

7.3 .3 PIC Limitations . . . . . . . . . . . . . . . . . . . . . . . . . . . . . . . . . . . . . . . . . . . .

7.4 Kinetic Model . . . . . . . . . . . . . . . . . . . . 151

7.4.1 Assumptions. . . . . . . . . . . . . . . . . . 151

7.4.2 Initial Distribution Function Requirements . . . . . . . . . . . . . 153

7.4 .3 Equation of the Model . . . . . . . . . . . . . . . . . . . 154

7.4 Bohm Criterion . . . . . . . . . . . . . . . . 156

7.4.5 High Potential . . . . . . . . . . . . . . . . . 158

7.4.6 Floating Potential . . . . . . . . . . . . . . . . 158

7.5 Conclusions . . . . . . . . . . . . . . . . . . . . 162

8 Conclusions and Future Work $\quad 165$

8.1 Ionization Stage . . . . . . . . . . . . . . . . . . . . . . . . . . . . . . . . . . . . . . . . .

8.2 Magnetic Electron Filtering Stage . . . . . . . . . . . . . . . . . 166

8.3 Extraction Stage . . . . . . . . . . . . . . . . 167

8.4 Future Work . . . . . . . . . . . . . . . . . . . . . 167

8.4.1 What Could Not Be Done . . . . . . . . . . . . . . . 167

8.4 PEGASES Prototype II . . . . . . . . . . . . . . . 168 
A Relation Between Edge and Average Electronegativity 


\section{Symbols and Abbreviations}

Symbol Description

$\alpha \quad$ electronegativity $\left(n_{-} / n_{e 0}\right)$

$\alpha_{0} \quad$ electronegativity at the center $\left(n_{-0} / n_{e 0}\right)$

$\alpha_{s} \quad$ electronegativity at $x=R\left(n_{-}(R) / n_{e 0}\right)$

$\bar{\alpha} \quad$ average electronegativity $\left(\bar{n}_{-} / n_{e 0}\right)$

a.m.u. atomic mass unit $\left(1.6605 \times 10^{-27} \mathrm{~kg}\right)$

$B_{0} \quad$ static magnetic field

$\beta \quad$ normalized ion velocity at $x=R\left(v_{ \pm}(R) / u_{B}\right)$

$c_{s} \quad$ sound speed

$D \quad$ diffusion coefficient

e elementary charge $\left(1.6022 \times 10^{-19} \mathrm{C}\right)$

$\varepsilon_{0} \quad$ vacuum permittivity $\left(8.8542 \times 10^{-12} \mathrm{Fm}^{-1}\right)$

$f \quad$ frequency

$f_{+} \quad$ positive ion distribution function

$f_{-} \quad$ negative ion distribution function

$h \quad$ positive ion edge-to-center density ratio

$K \quad$ reaction rate

$m_{+} \quad$ positive ion mass

$m_{-} \quad$ negative ion mass

$m_{e} \quad$ electron mass

$M_{\text {eff }} \quad$ mass efficiency $\left(\Gamma_{+} /\left.\Gamma_{n}\right|_{\text {exit }}\right)$

$\mu \quad$ mobility coefficient

$\nu \quad$ collision frequency

$n_{+} \quad$ positive ion density

$n_{-} \quad$ negative ion density

$n_{e} \quad$ electron density

$n_{g} \quad$ neutral density

$\varphi \quad$ potential

$p \quad$ pressure

$P \quad$ power

$r \quad$ radius

sccm standard cubic centimeters per minute

$T_{+} \quad$ positive ion temperature

$T_{-} \quad$ negative ion temperature

$T_{e} \quad$ electron temperature

$u_{B} \quad$ Bohm velocity

$v_{+} \quad$ positive ion velocity

$v_{-} \quad$ negative ion velocity

$v_{e} \quad$ electron velocity

$\omega \quad$ angular frequency 


\section{Chapter 1}

\section{Introduction}

\section{Contents}

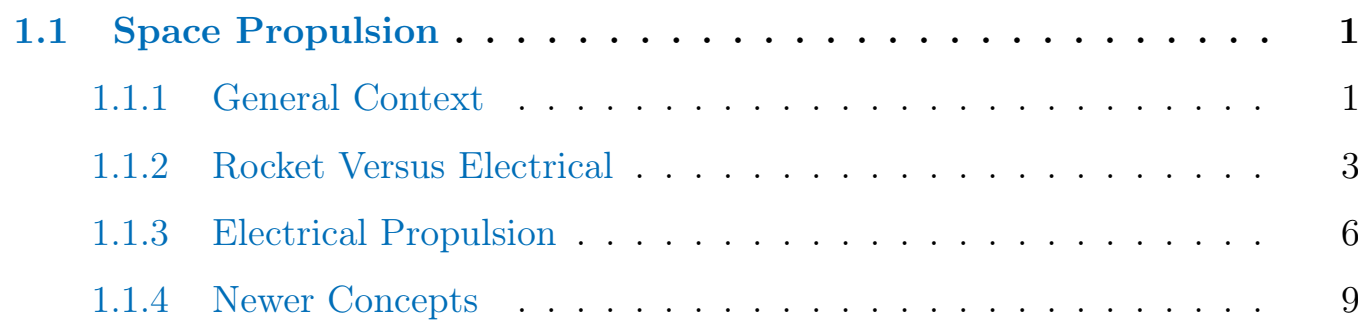

1.2 PEGASES: Plasma Propulsion with Electronegative GASES 10

1.2 .1 The Idea . . . . . . . . . . . . . . . . . . . 10

1.2 .2 Advantages of PEGASES . . . . . . . . . . . . . 13

1.2.3 Timeline and Financing . . . . . . . . . . . . . . 13

1.3 Outline ........................ 14

\subsection{Space Propulsion}

\subsubsection{General Context}

Space, or outer space, is defined as the relatively empty regions in the universe between celestial bodies. In the case of planets surrounded by an atmosphere, the latter is of course considered part of the planet and not space. It is also a region that mankind has wanted to explore for as long as Man has looked up from the ground. Contrary to the popular belief that it is a perfect vacuum, it contains atoms and particles with a density around ten per cubic centimeter [1]. For comparison, the density of air at sea level for a temperature of $20^{\circ} \mathrm{C}$ is $2.5 \times 10^{19}$ per cubic centimeter.

There are many reasons to send objects in space. Because of the atmosphere turbulence and city lights, observations of space are difficult and have limited accuracy. These issues can be solved by sending a telescope to space, as was the case with the Hubble Space Telescope [2]. Once in space, such a telescope can study the Earth (photos, meteorology, etc.), other celestial bodies (planets in our solar system, other solar systems, galaxies, etc.) or space itself (density of particles, radiations, etc.). An object orbiting a planet is called a satellite whether it is natural, e.g. Moon orbiting the Earth, or man-made, 
e.g. Hubble Space Telescope. A satellite can also be used for telecommunications: phone, television, GPS system, etc.

To achieve these objectives, a method to put the object in space and then move it, referred to as space propulsion, is needed. Any movement is described by Newton's laws [3]:

- Every body persists in its state of being at rest or of moving uniformly straight forward, except insofar as it is compelled to change its state by force impressed,

- The change of momentum of a body is proportional to the impulse impressed on the body, and happens along the straight line on which that impulse is impressed,

- For a force there is always an equal and opposite reaction: or the forces of two bodies on each other are always equal and are directed in opposite directions.

In a simpler form, these laws state that for an object to move, it has to push on another object. For instance, a pedestrian is able to walk because he is pushing on the ground with the combination of gravity keeping him on it and friction that allows him to create a force parallel to the ground. Any mean of propulsion works in the same way: cars with tires on the ground, boats with propellers on water or wind on sails, etc. As space is nearly empty (no ground to push on), these classical methods of propulsion cannot work.

To solve this problem, one has to look at the third Newton law. On Earth, as an object is thrown, the thrower appears not to move. This is because the force impressed on him is transmitted to the ground through friction. In space, the sender is not held and will move in the opposite direction of the thrown object. This effect can be seen when two skaters (weak friction on the ground) push on each other and end up going away from one another. Intuitively, the faster the object is thrown, the faster the sender moves in the opposite direction. This is the principle on which space propulsion operates: a mass is accelerated and ejected from the vehicle. As a consequence, a part of the mass of the vehicle, called propellant, is used for propulsion.

Assuming that the variation of the total mass of the vehicle is negligible compared to the initial total mass $M$, the second Newton law can be written as

$$
\text { Force }=M \frac{d v}{d t},
$$

with $v$ the velocity of the vehicle and $t$ time. Using the same law, the force on the vehicle $T$ called thrust can be expressed as

$$
T=-\frac{d}{d t}\left(m_{p} v_{\mathrm{ex}}\right)
$$

with $m_{p}$ the mass of the propellant and $v_{\text {ex }}$ the velocity of the ejected propellant compared to the vehicle. To simplify the problem, the exhaust velocity is assumed to be constant, resulting in

$$
T=-v_{\mathrm{ex}} \frac{d m_{p}}{d t}
$$

The mass of the spacecraft can be separated into two categories. On the one hand, the propellant which will be ejected. On the other hand, the body of the spacecraft and its cargo, also called the payload. The mass of the latter being constant,

$$
\frac{d M}{d t}=\frac{d m_{p}}{d t}
$$


Combining (1.1), (1.3) and (1.4) yields

$$
M \frac{d v}{d t}=-v_{\mathrm{ex}} \frac{d M}{d t}
$$

which can be rewritten as

$$
d v=-v_{\mathrm{ex}} \frac{d M}{M} .
$$

For a straight line motion, this equation can be integrated from the initial state $\left(v_{i}, M_{i}\right)$ to the final state $\left(v_{f}, M_{f}\right)$. Defining $\Delta v=v_{f}-v_{i}$ as the increase in velocity, the integration gives

$$
\Delta v=v_{\mathrm{ex}} \ln \frac{M_{i}}{M_{f}}
$$

The specific impulse $\mathrm{I}_{\mathrm{sp}}$ is defined as the ratio of the propellant exhaust velocity $v_{\mathrm{ex}}$ to the gravitational acceleration $g$ [4]. Equation (1.7) can thus be rewritten as

$$
\frac{\Delta v}{g}=\mathrm{I}_{\mathrm{sp}} \ln \frac{M_{i}}{M_{f}} .
$$

It can be seen from this equation that there are two ways to maximize the increase in velocity: either by increasing the exhaust velocity, or the mass expelled (ratio $M_{i} / M_{f}$ ).

\subsubsection{Rocket Versus Electrical}

Two main propulsion methods can be used. The first is called rocket propulsion and relies on the fast expansion of a heated gas, channeled into the proper geometry. The second, electrical propulsion, consists of creating a plasma composed of ions and electrons where the ions are accelerated outward by electromagnetic forces (potential difference between two plates acting on ionic charges in the simplest case).

\section{Rocket Engine}

The heated gas for rocket propulsion can be created with a multitude of designs [5], with three as the main ones:

- chemically powered: two propellants or more, whether solid or liquid, react together to create the heated gas. One propellant is a fuel and the other one an oxidizer,

- electrically powered: a monopropellant is heated electrically either directly or through an arc,

- nuclear powered: the nuclear reaction is used to heat the propellant.

To optimize the thrust created by the heated gas, a nozzle is placed at the exit of the combustion chamber. The nozzle is the element converting most of the thermal energy into kinetic energy. The exiting gas velocity is a few kilometers per second.

As it can be seen on figure 1.1a, the expansion of the heated gas results in pressure forces (gray arrows) on all surfaces of the combustion chamber and the nozzle. Any pressure force perpendicular to the gas exhaust does not provide thrust (no outline) as it is compensated by an opposite one. Parallel to it, a positive thrust is provided (blue 


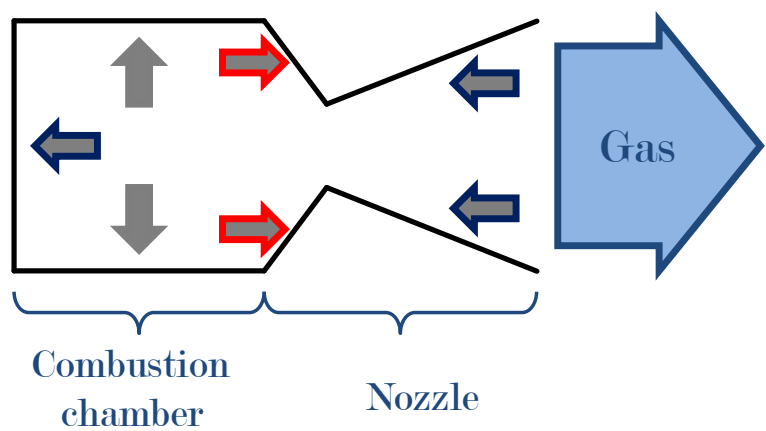

(a)

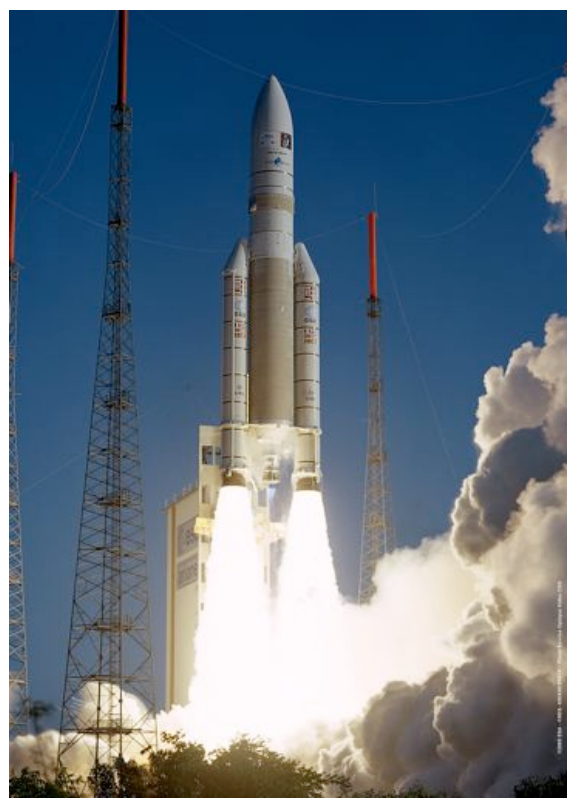

(b)

Figure 1.1: Rocket principle (a) with the expansion of the heated gas (gray arrows): part of the pressure does not provide thrust (no outline), part does (blue outline) and part provides a negative thrust (red outline). Ariane 5 (b) combines several rocket engines to put satellites in orbit [credits Ariane Espace].

outline) when the force is opposite to the gas exhaust, and negative (red outline) when in the same direction.

Ariane 5, figure 1.1b, combines several rocket engines to take its payload from ground to orbit. Moreover, it is separated in different stages that burn one after the other. For its main core stage [6], liquid hydrogen (fuel) and liquid oxygen (oxidizer) are used as propellants, and fed in the combustion chamber with two turbo-pumps. A thrust of $1390 \mathrm{kN}$ is thus provided in vacuum with a specific impulse of $432 \mathrm{~s}$. As much as $170 \mathrm{t}$ of propellant is ejected in $540 \mathrm{~s}$.

\section{Electrical Propulsion}

A plasma is a collection of charged particles, with or without a neutral background, moving freely in a volume. In equilibrium, as electrical forces interact with the particles, a plasma is neutral on average. It is created by applying enough power to a gas to partially or fully ionize it (an atom is separated into an ion and an electron in the general case). Since only ions are used to create thrust, the process is optimized when the plasma is fully ionized: any neutral entering the chamber is turned into an ion, accelerated and provides thrust. The electrical power can either be direct-current or alternating-current. As thrusters are meant to be operated in space, the background pressure is zero. The pressure in the chamber resulting from the gas injection is thus low: plasmas in electrical thruster are low-pressure plasmas. In the case of an alternating-current electrical power, these plasmas can be created via three main modes of power coupling [7], shown in figure 1.2: capacitive (a), inductive (b) or wave heated (c).

The classical geometry of a direct-current discharge consists of a positive anode at one end and a negative cathode at the other. When the constant electric field between the 


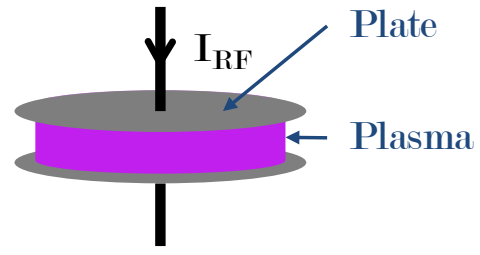

(a)

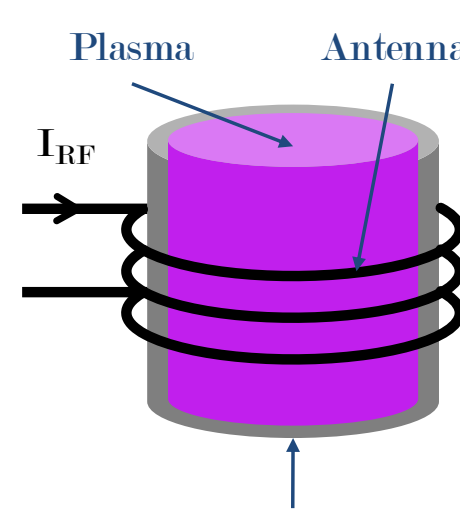

Dielectric tube

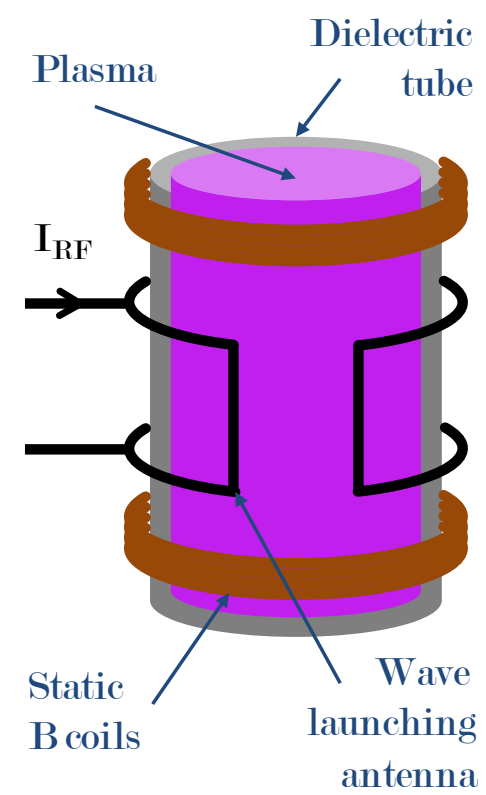

(b)

(c)

Figure 1.2: Modes for plasma creation with an alternative-current electrical power: capacitive (a), inductive (b) and wave heated (c) in the case of a helicon wave which needs a static magnetic field to be launched.

two electrodes is high enough, the neutral gas is ionized and a plasma is created. The advantage of these discharges, compared to alternating-current ones, is that all plasma parameters are constant, and thus more easily investigated. However, the structure of the discharge is quite complicated.

The simplest structure of a capacitively coupled discharge consists of two plates surrounding a volume where a gas is introduced. The applied power creates an electric field between the two plates. When this electric field is strong enough to ionize the neutral gas, a plasma is created. As a plasma is quasi-neutral, most of the potential difference occurs in a small region, called a sheath, near the polarized plates. The plasma density obtained is of the order of $10^{16} \mathrm{~m}^{-3}$. For the discharge to be considered capacitive, the characteristic length of the plates should be small compared to the vacuum wavelength of the electric field when the applied power is alternating [8]. Otherwise, a standing wave effect occurs [9] with the consequence of the induced magnetic field playing a role in the discharge equilibrium [10], as in an inductively coupled discharge.

In an inductively coupled discharge, the magnetic field induced by a current creates and sustains the plasma. The applied power is coupled to the plasma with a coil which can be either around the plasma volume or placed next to one of its containment walls. This results in the creation of an evanescent wave in the plasma. The walls of the plasma volume should be made out of dielectric material to allow the electromagnetic field to penetrate. The plasma density about $10^{17}-10^{18} \mathrm{~m}^{-3}$ is higher than capacitively coupled discharges. It should be noted that in the case where the antenna is close to the wall, a capacitive coupling does occur, resulting in a simultaneous capacitive and inductive power coupling.

The last mode of power coupling is the wave heated discharge. The waves can either be created outside of the plasma, as in microwave discharges [7], or created directly in the 
plasma itself by another mode, such as helicon discharges [11, 12]. In the case of a helicon discharge, a static magnetic field is necessary for the wave to exist in the plasma. Several helicon modes exist $[13,14]$ and require specifically designed antennae to be efficiently excited. The typical plasma density around $10^{18}-10^{19} \mathrm{~m}^{-3}$ is higher than both previously discussed modes.

The electrical nature of the ion acceleration allows high exiting velocities, therefore a high $\mathrm{I}_{\mathrm{sp}}$, to be reached. However, as the neutral gas is used at low pressure, the expelled mass is small. The resulting thrust is usually low with a maximum around $1 \mathrm{~N}$. As an example, the Hall thruster BPT-4000 [15] at a maximum power of $4.5 \mathrm{~kW}$ delivers a thrust of $254 \mathrm{mN}$ with an $\mathrm{I}_{\mathrm{sp}}$ of $2150 \mathrm{~s}$.

\section{Comparison}

Rocket and electrical propulsion have different characteristic values: high thrust and low $\mathrm{I}_{\mathrm{sp}}$ for the first, and low thrust and high $\mathrm{I}_{\mathrm{sp}}$ for the second. Therefore, on average, they are used for different types of missions.

As a rocket engine expels a lot of propellant, it cannot be used for long term missions where the weight and volume of the stored propellant would be too large. However, it is the only method of bringing an object from ground to orbit with thrusts ranging as high as a few meganewtons.

The situation for electrical thrusters is the opposite as the thrust is very small compared to a rocket engine. Nevertheless for a small weight and volume of stored propellant, this low thrust can be sustained for an extended time. Electrical thrusters are well suited for long missions.

In the case of sustaining a satellite in orbit around Earth where friction forces due to the atmosphere need to be compensated, either propulsion method is reasonable since the required thrust is low and the propellant usage scarce enough for a rocket engine not to be cumbersome.

However, there is a significant drawback to electrical propulsion: the electrical power needed to operate electrical thrusters is not directly available from the power conversion systems on satellites and probes [16]. These systems convert the power collected by solar panels into usable power at given voltages and currents. Depending on the type of electrical thruster, the demand in voltage or current is too high for classical power conversion systems. An additional power conversion subsystem, therefore, is needed which adds weight to the spacecraft. As the mass of the power conversion subsystem depends on the amount of power required, an efficiency parameter is the mass to available power ratio called specific mass in $\mathrm{kgkW}^{-1}$, multiplying the classical efficiency of a power supply (ratio of delivered power to consumed power). In the case of Hall thrusters, specific masses can be as high as $10 \mathrm{kgkW}^{-1}$ for power efficiencies as high as 93\%. Again, as voltage and current requirements depend on the type of thruster, the power efficiency also depends on it: for ion thrusters, the maximum power efficiency is around $88 \%$.

\subsubsection{Electrical Propulsion}

As described previously, the electrical propulsion relies on the creation of a plasma where the positive ions are accelerated and expelled to create thrust. Two main categories of electrical thrusters are currently being used for space missions: the ion thruster and the Hall thruster. 


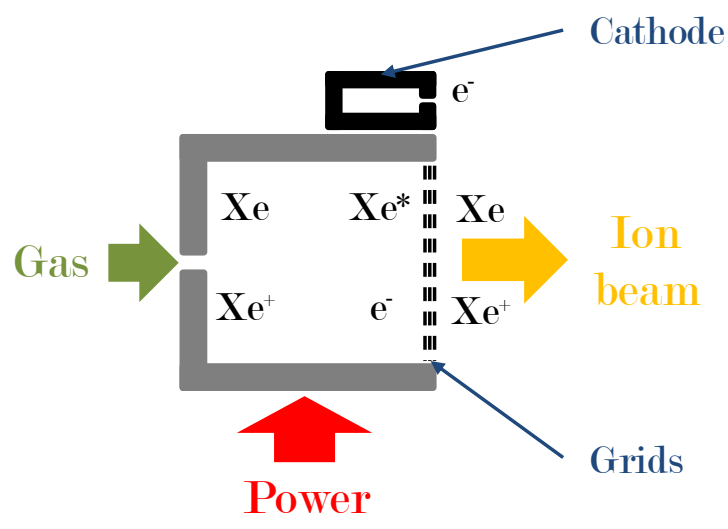

(a)

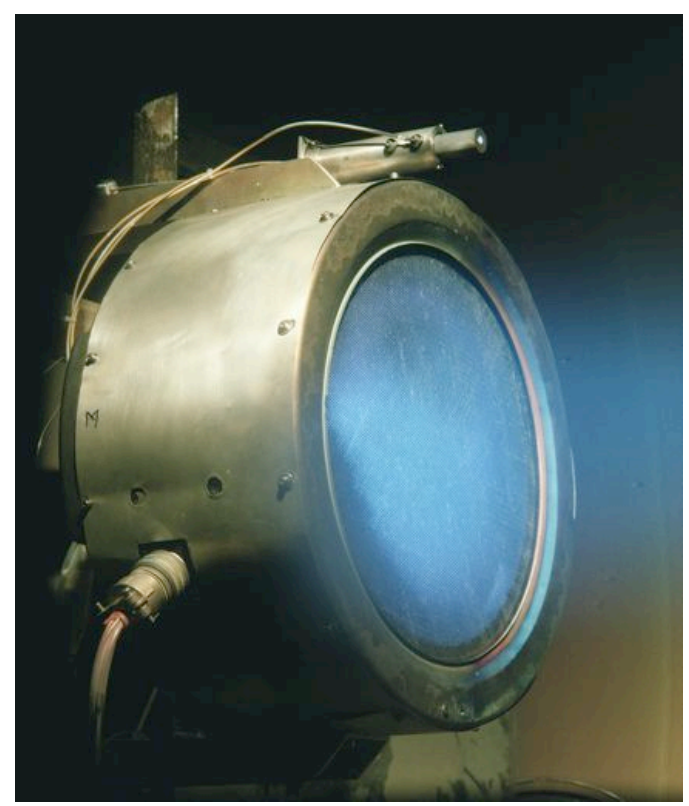

(b)

Figure 1.3: Ion thruster principle (a) with three stages: plasma creation, ion acceleration (grids) and neutralization (cathode). Picture of an ion thruster (b) with the exiting ion beam [credits NASA JPL].

\section{Ion Thruster}

Three stages characterize an ion thruster: plasma creation, ion acceleration and neutralization. These can be separated in three different regions of the thruster as can be seen schematically in figure 1.3a.

The different modes possible to couple energy to a plasma were presented in section 1.1.2. The electrical power can either be direct-current or alternating-current. The aim of the first stage is to obtain a fully ionized plasma for a minimum power, with the ideal situation of every atom or molecule converted into an ion. However, an atom can also be excited without separating into an ion and an electron. The two types of reactions possible can be written in the case of xenon (a widely used propellant for electrical thrusters) as

$$
\begin{aligned}
& \text { Ionization : } \mathrm{Xe}+\mathrm{e}^{-} \rightarrow \mathrm{Xe}^{+}+2 \mathrm{e}^{-} \\
& \text {Excitation }: \mathrm{Xe}+\mathrm{e}^{-} \rightarrow \mathrm{Xe}^{*}+\mathrm{e}^{-}
\end{aligned}
$$

Both processes are functions of the electron energy. For Maxwellian electrons and a temperature below $8 \mathrm{~V}\left(9.3 \times 10^{4} \mathrm{~K}\right)$, the excitation rate exceeds the ionization rate. This means that the ionization process is fairly inefficient for such electron temperatures. The design for the power coupling should minimize the part used for excitation by producing electron temperatures as high as possible, or non-Maxwellian distributions with enhanced tails of high energy electrons. One containment method is to employ a magnetic field cusp, using permanent magnets around the plasma volume.

Once the ions are created, they need to be accelerated, thus forming an ion beam which can be seen in figure 1.3b. Although not the only possibility, the main method of ion acceleration consists of biased grids. This mechanism relies on the fact that a charged 
particle is accelerated in a steady electric field: potential energy is transformed into kinetic energy. A minimum of two grids is necessary: the screen grid and the acceleration grid. The role of the first grid is to ensure that no electrons enter the acceleration region where they would be accelerated back into the plasma region and damage the thruster. The potential difference between the two grids accelerates the ions outward. As grids are constantly bombarded, the choice of material is important for a long thruster lifetime: a low sputter erosion rate for the given propellant is needed. The main materials used are molybdenum, carbon-carbon composites and pyrolytic graphite. Two erosion mechanisms occur on the accelerating grid: the barrel erosion and the erosion due to secondary ions. As ions are accelerated, a part of them is collected by the accelerating grid resulting in barrel erosion: the edges of the grid holes are sputtered. At the same time, ions that left the thruster can undergo charge exchange collisions yielding a fast neutral and a slow ion called secondary ion. This slow ion, called a backscattering ion, is then accelerated toward the thruster because of the bias of the accelerating grid. A third grid positioned after the accelerating grid collects these secondary ions and preserves the accelerating grid, increasing its lifetime.

The fact that positive ions are leaving the thruster leads to a negative charging of the thruster body, which would end up in attracting the expelled ions, canceling the thrust. The exiting ion beam, therefore, needs to be neutralized, which is realized by an electron emitting hollow cathode placed at the thruster exit. There are thus two charged beams leaving the thruster, one positive and one negative. The cathode is a vital part of the thruster, and several materials and geometries are used depending on the conditions.

\section{Hall Thruster}

Contrary to ion thrusters where the three stages correspond to three different regions, a Hall thruster combines all of these into one chamber. It is a gridless thruster, which, therefore, eliminates the grid lifetime issue of ion thrusters. The characteristic specific impulse is usually lower than that of ion thrusters, but the absence of current limitations due to grids makes its thrust capabilities similar to ion thrusters.

The geometry of a Hall thruster is cylindrical with its axis in the direction of the thrust, see figure 1.4a. The plasma region is annular, as can be seen in figure 1.4b, and is set between an anode where the gas is introduced and a cathode at the exit of the thruster. Contrary to ion thrusters, the cathode does not only act as a neutralizer for the exiting ion beam, but is also part of the discharge mechanism. A part of the electrons emitted by the cathode feeds the channel where the plasma is created and sustained; another part flows with the exiting ion beam for charge neutrality to be fulfilled. The magnets, placed at the center and outside of the thruster body, create a radial static magnetic field. Given the mass ratio between electrons and ions, an intermediate value of the magnetic field confines the electrons (Larmor radius smaller than the system) but not the ions (Larmor radius bigger than the system). As the diffusion of electrons is impeded by the magnetic field, the potential difference between the anode and the cathode is distributed and electrons are not accelerated toward the anode. Instead, electrons have a helical motion in the channel due to the $\mathbf{E} \times \mathbf{B}$ drift. Ions created in the plasma are thus accelerated outward and provide thrust.

Similarly to ion thrusters, two main processes occur: ionization (1.9) and excitation (1.10). The aim is once again to reduce the power dissipated in excitation to optimize the thruster. The electron temperature is higher in the channel and estimated 


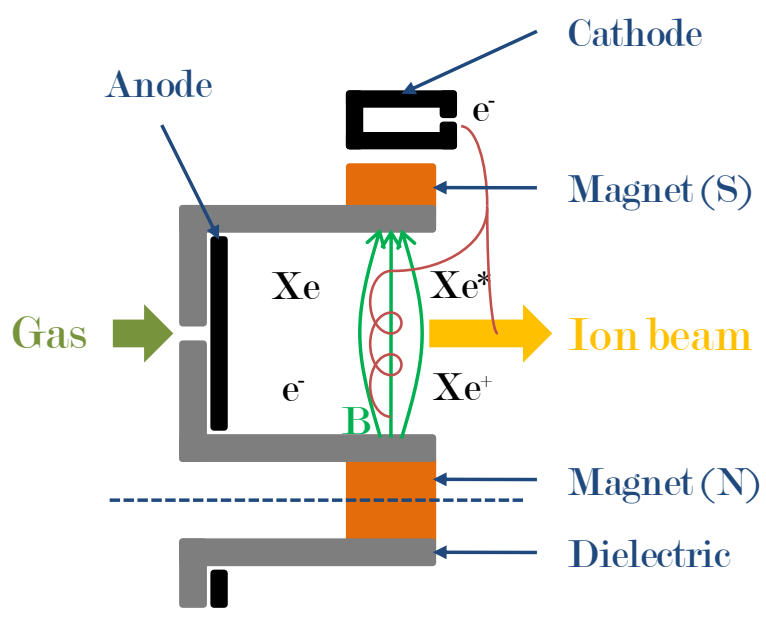

(a)

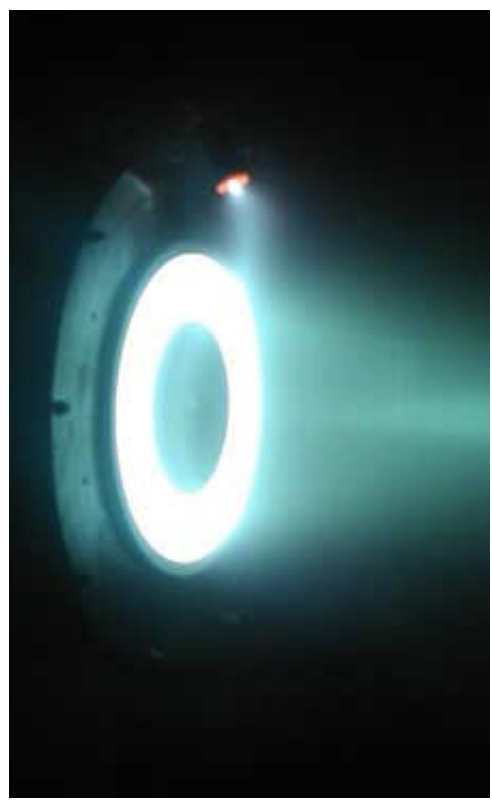

(b)

Figure 1.4: Schematics (a) of a Hall effect thruster: the plasma is created between the anode and the cathode with electrons confined along the magnetic field lines. Photo (b) of a Hall effect thruster.

around $25 \mathrm{~V}\left(2.9 \times 10^{5} \mathrm{~K}\right)$, which results in a better ionization to excitation ratio.

A Hall thruster lifetime is limited by the erosion of the channel wall (dielectric) and the cathode lifetime. The material for the channel wall should have a low sputter erosion rate with the propellant, xenon in most cases. A widely used material is $\mathrm{BNSiO}_{2}$.

\subsubsection{Newer Concepts}

Concepts other than the ion thruster and the Hall effect thruster are being developed, but are yet to be used on actual probes or satellites. Some aim at higher power to obtain higher thrusts (VASIMIR), and others on a physical effect present in plasmas to create a gridless and anodeless thruster (HDLT).

\section{Variable Specific Impulse Magnetoplasma Rocket}

The VASIMIR thruster was invented by F R Chang-Diaz and has been in development since 1979 [17]. This thruster is composed of three stages: the plasma is first created with a radio-frequency antenna, then heated with a radio-frequency booster, and finally a magnetic nozzle transforms the energetic ions into fast moving ions.

In order to reach the highest plasma densities possible, a helicon mode of power coupling was chosen with as much as $30 \mathrm{~kW}$ delivered to the antenna. The radio-frequency booster consists of an ion cyclotron resonance: the ions are magnetized with a strong magnetic field, and an electromagnetic wave with a period equal to the period of rotation of the ions around the magnetic field lines is applied. The resulting local electric field is always in the same direction as the ion motion, accelerating the ions which end up with a high azimuthal velocity. A magnetic nozzle consists of a strong magnetic field divergence. As the strength of the magnetic field decreases, the azimuthal velocity of the ions 
is transformed into axial velocity (magnetic moment and energy conservation) [7].

The VASIMIR thruster is being tested with different propellants [18] and a recent agreement with NASA allows future tests on the International Space Station.

\section{Helicon Double Layer Thruster}

The HDLT was invented by C Charles and R W Boswell with a patent filed in 2002 [19]. As a gridless and cathodeless thruster, it removes two main lifetime limitations of thrusters.

This thruster is also based on a helicon power coupling for the creation of the plasma. The radio-frequency used for the first prototype was $13.56 \mathrm{MHz}$. The set of coils creates a magnetic field not only for the helicon wave to exist in the plasma, but also provides a magnetic field divergence at the exit of the thruster. This divergence is a possible condition for the appearance of a double layer. The latter is defined [20] as two equal but oppositely charged, essentially parallel but not necessarily plane, space charge layers. The regions around it have to satisfy quasi-neutrality. The potential profile over a double layer, therefore, is an elongated step function (Poisson equation). The double layer in the HDLT appears with a negative potential difference between where the plasma is created and the thruster exit: ions going through the double layer as they diffuse are accelerated, which makes the HDLT a gridless thruster. Moreover, as the downstream plasma is quasi-neutral, there is no need for neutralization: the HDLT is a cathodeless thruster.

The main limitation of this thruster concept comes from the relatively small potential difference over the double layer. As this potential difference is responsible for the acceleration of the ions, the specific impulse of this thruster concept is limited.

The concept of the HDLT was studied at Laboratoire de Physique des Plasmas (LPP) during Nicolas Plihon's PhD thesis [21] in the context of an ESA report [22].

\subsection{PEGASES: Plasma Propulsion with Electronega- tive GASES}

\subsubsection{The Idea}

The PEGASES thruster is a very recent electrical thruster concept developed in the Laboratoire de Physique des Plasmas (LPP), with a patent [23] filed in late 2005. Its principle is a consequence of studies done on a helicon reactor with electronegative gases. An electropositive gas is a gas that, in a plasma state, yields positive ions and electrons. Examples of such gases are argon (Ar), krypton (Kr) and xenon (Xe). The ionization reaction that creates the plasma can be written as

$$
\mathrm{Ar}+\mathrm{e}^{-} \rightarrow \mathrm{Ar}^{+}+2 \mathrm{e}^{-}
$$

for an argon plasma. The plasma equilibrium results of the interaction of two species, the positive ions and the electrons. An electronegative gas yields positive ions, negative ions and electrons. Oxygen $\left(\mathrm{O}_{2}\right)$ and sulfur hexafluoride $\left(\mathrm{SF}_{6}\right)$ are examples, among others. Two processes take part in the creation of the plasma: ionization and attachment. The main ionization reaction is similar to electropositive gases:

$$
\mathrm{O}_{2}+\mathrm{e}^{-} \rightarrow \mathrm{O}_{2}^{+}+2 \mathrm{e}^{-},
$$


in the case of oxygen. The attachment reaction needs a molecular gas as electrons are too energetic to attach directly to a neutral. Part of the electron energy is absorbed by the molecule, which either breaks down into atoms and molecules or transforms the electron energy into rotational and vibrational oscillations. The breaking down reaction is called dissociative attachment:

$$
\mathrm{O}_{2}+\mathrm{e}^{-} \rightarrow \mathrm{O}^{-}+\mathrm{O}
$$

for oxygen. Otherwise, it is direct attachment:

$$
\mathrm{SF}_{6}+\mathrm{e}^{-} \rightarrow \mathrm{SF}_{6}^{-}
$$

for sulfur hexafluoride. In the simple case of oxygen, there are three charged species that interact for the plasma equilibrium, one positively charged and two negatively charged. For bigger molecules, several species of positive ions and negative ions can be created, giving a more complex equilibrium. As a result, electronegative plasmas are prone to instabilities [24, 25, 26].

Since the power is coupled via a helicon mode, a static magnetic field is necessary and modifies the behavior of charged species as a circular motion is superimposed on their trajectories. The radius of such circular motions is called the Larmor radius and can be expressed as

$$
r_{L}=\frac{m v_{\perp}}{|q| B}
$$

with $m$ the particle mass, $v_{\perp}$ its velocity perpendicular to the magnetic field, $q$ its charge and $B$ the strength of the magnetic field. Comparing the Larmor radius of a species and the characteristic length of the plasma volume gives a rough indication whether it is magnetized or not. As positive and negative ions have comparable masses, there are two characteristic Larmor radii in an electronegative plasma, that of the electrons and that of the ions. The mass ratio between electrons and ions $\left(5.8 \times 10^{4}\right.$ for $\left.\mathrm{O}_{2}^{+}\right)$is such that the Larmor radius for electrons is much smaller than the one for ions. Therefore, it is possible to choose a magnetic field strength for a given characteristic dimension where electrons are magnetized but ions, both positive and negative, are not. Considering the cylindrical geometry of a helicon reactor, the electrons may be confined to the center where they are created, while ions may diffuse more rapidly across the magnetic field. An electron-free plasma, or ion-ion plasma, may be created in the periphery of the electronegative core. This is possible since quasi-neutrality can be fulfilled with two oppositely charged species. A steady-state ion-ion plasma can be created in this way.

The idea behind PEGASES consists of using both positive and negative ions for thrust, contrary to classical thrusters using positive ions only as can be seen in figure 1.5ab. The general structure of PEGASES is similar to a classical electropositive thruster: a propellant is ionized by applying electrical power and the ions are accelerated with an electrical method to create the thrust. However, there are many subtleties. First, an electronegative propellant is chosen and yields three species: positive ions, negative ions and electrons. Second, between the plasma creation and the ion acceleration is an additional stage where the electrons are filtered by a static magnetic field. Third, both ion species are accelerated to provide thrust. After different acceleration methods were considered, biased grids were chosen and will be discussed in chapter 7. Finally, there is no need for neutralization with a cathode as the accelerated beam is neutral to begin with. In order to maximize the plasma density, a helicon mode is chosen as the power coupling method. 


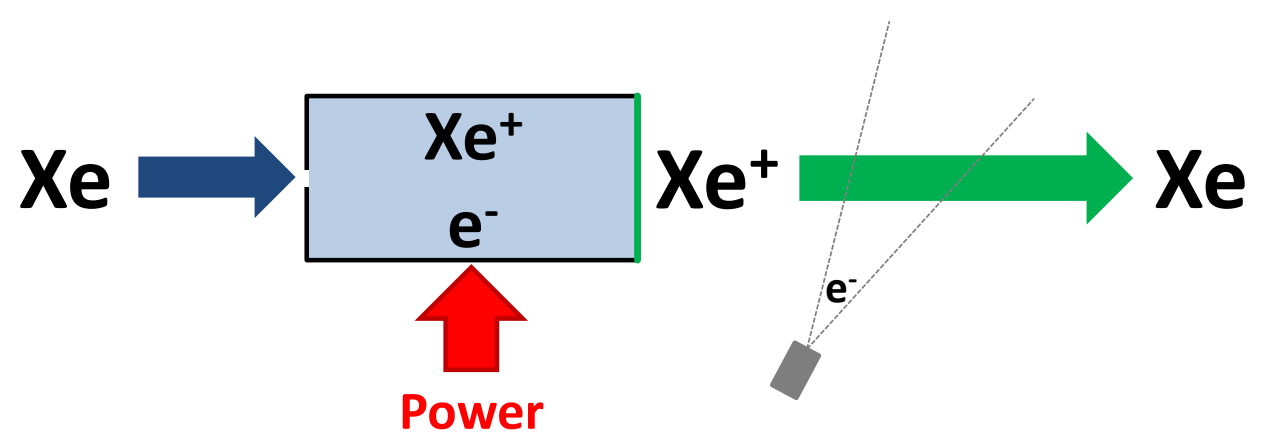

(a)

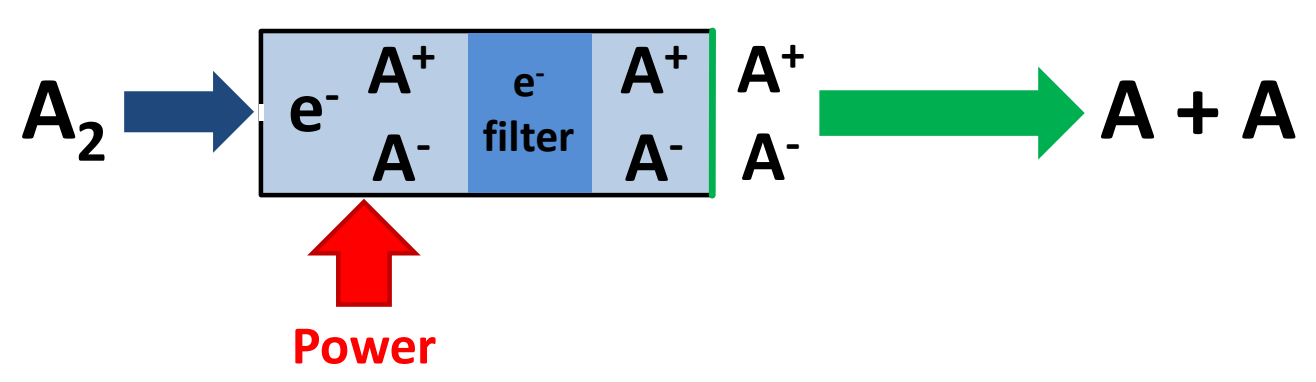

(b)

Figure 1.5: Principle of an electropositive plasma thruster (a) with xenon as propellant and principle of the PEGASES thruster (b) with $A_{2}$ as electronegative propellant. 


\subsubsection{Advantages of PEGASES}

Not only is PEGASES a revolutionary thruster concept because it uses both positive and negative ions for thrust, but it also possesses key advantages compared to classical electropositive thruster.

As described above (1.2.1), the ion beam created for the thrust is neutral, rendering a neutralizing cathode unnecessary. Therefore, the PEGASES thruster lifetime is not limited by the cathode lifetime. Compared to Hall thrusters, the elimination of the cathode ensures that no electrons are accelerated back toward the plasma chamber, which is a source of wall erosion, and a thruster breakdown possibility.

In an electropositive thruster, the process of neutralizing the ion beam with electrons should end with the recombination between these two species, but in the case of PEGASES, the recombination happens between positive and negative ions. The velocity component of ions contributing to the thrust is along the thruster axis, with any other component either useless or deteriorating the total thrust. For optimization, the beam should be neutralized as soon as possible as a singly charged beam will diverge due to electrical forces, the same charge particles repelling one another. As the recombination between positive ions and negative ions is significantly faster than that of positive ions and electrons, a beam divergence is less likely to happen for the PEGASES thruster. For comparison in the case of oxygen, the reaction rate for the recombination between $\mathrm{O}_{2}^{+}$and an electron is $2.2 \times 10^{-14} / \sqrt{T_{e}} \mathrm{~m}^{3} \mathrm{~s}^{-1}$, and for the recombination between $\mathrm{O}_{2}^{+}$ and $\mathrm{O}^{-}, 5.2 \times 10^{-14}\left(0.026 / T_{i}\right)^{0.44} \mathrm{~m}^{3} \mathrm{~s}^{-1}$. The ratio between the two constant factors of these reaction rates is 2.5 . The temperature dependence further increases the ratio of ion-ion recombination to electron-ion recombination: for the typical values of $T_{e}=3 \mathrm{~V}$ $\left(3.5 \times 10^{4} \mathrm{~K}\right)$ and $T_{i}=26 \mathrm{mV}(300 \mathrm{~K})$, the ratio becomes 4.1 . For an electron temperature of $10 \mathrm{~V}\left(1.2 \times 10^{5} \mathrm{~K}\right)$ and the same ion temperature, the ratio is 7.5 . It should be noted that the ion-ion recombination rate is even faster for other gases like sulfur hexafluoride $\left(\mathrm{SF}_{6}\right)$ and dichlorine $\left(\mathrm{Cl}_{2}\right)$.

When grids are used to accelerate ions, the grid lifetime is limited by erosion due to backscattering ions, described in section 1.1.3. This process is also limited by how fast ions are neutralized. Since the recombination for the PEGASES thruster should be significantly faster, the grid lifetime should be increased in the case of a PEGASES thruster using grids for ion acceleration.

\subsubsection{Timeline and Financing}

The PEGASES thruster project began with the filing of a patent by Pascal Chabert on December 7, 2005 [23]. This patent describes the basic idea of this new thruster concept (see 1.2.1). At that time, no experiments or modeling had been done.

I began working on this project in March, 2006, for a four month long internship which was part of my Plasma Physics Master Degree. I investigated ion-ion plasmas created in the helicon reactor and worked on the early beginning of sheath modeling for ion-ion plasmas.

I obtained a three year PhD grant in May, 2006, to begin in September, 2006. This grant was financed by Ecole Polytechnique and required me to spend a minimum of two months abroad for a collaboration. Concurrently, an ANR Jeunes Chercheurs grant was awarded to Pascal Chabert, to be used for this project. Two Post-Docs were recruited for PEGASES related work. Ane Aanesland obtained a Marie Curie grant (Europe) for a du- 
ration of two years on the experimental aspects of the PEGASES thruster. Albert Meige, through the ANR Jeunes Chercheurs, worked for eighteen months on PIC simulations.

The first year of the project (September 2006 - August 2007) was spent designing the first PEGASES prototype and ion-ion plasma sheath modeling with fluid models, PIC simulations and kinetic models. A group of students from Ecole Polytechnique took part in both aspects of the PEGASES project for their Projet Scientifique Commun (PSC), with two hours per week dedicated to it. I supervised two students working on the experiments and a third one on ion-ion plasma sheath modeling. A visit to Pivoine $2 \mathrm{G}$, located in the ICARE laboratory, was organized. It consists of a large test chamber for thrusters where different diagnostics, among which a thrust scale, can be used to characterize a thruster model. One of the tested thrusters was the Hall thruster PPS1350-G which was chosen as the propulsion system on the Smart-1 mission coordinated by the European Space Agency. By the end of the year, the first PEGASES prototype was assembled, along with its vacuum chamber and electrical diagnostics.

During the second year (September 2007 - August 2008), the PEGASES prototype was characterized, as well as evolutions of the first design. The kinetic study of ion-ion plasma sheaths was continued. Another group of students from Ecole Polytechnique picked up where the previous one had left off: experiments on the PEGASES prototype and ion-ion plasma modeling. I once again supervised two students working on the experimental part of the project. As was required by my $\mathrm{PhD}$ grant, I spent ten weeks (between March and May) at UC Berkeley, California, to work with Allan J Lichtenberg and Michael A Lieberman from the Electrical Engineering and Computer Science department at UC Berkeley, on the magnetic electron filtering in electronegative plasmas to obtain an ion-ion plasma. Fluid models were developed in the case of an oxygen plasma. During this year, EADS-Astrium, a european company in space propulsion, took interest in the PEGASES thruster, bought the patent back from Ecole Polytechnique and started a collaboration with the laboratory.

The third year (September 2008 - August 2009) saw the continuation of experiments on the PEGASES prototype and further evolutions of its design. The collaboration with UC Berkeley on the magnetic electron filtering went on. On November 28, a new patent on the PEGASES thruster [27] was filed by Pascal Chabert, Ane Aanesland, Albert Meige and me. In the context of the collaboration, it was paid for by EADS-Astrium. At the start of the year, Ane Aanesland became a permanent researcher in the laboratory on the PEGASES thruster, specifically on the experimental part. Also, Lara Popellier started working in March on the experimental aspects of PEGASES during an internship, part of her Plasma Physics Master Degree. She will be picking up the work on the PEGASES project from where I left off during her PhD thesis to start in September, 2009.

\subsection{Outline}

First, a short background on plasma physics is presented in chapter 2, with the various models and diagnostics which were used for this thesis. The models range from PIC simulations, through kinetic models, fluid models, to global models. The notations are also defined. Two types of diagnostics were used, Langmuir-type probes and a retarding field energy analyzer. Electronegativity measurement techniques are also presented, with an emphasis on the electrostatic probe technique, used in this thesis.

The PEGASES thruster can be divided into three stages: the ionization stage, the 
magnetic electron filtering stage, and the ion acceleration stage. These stages form the structure of this thesis, which moreover, is divided into experimental results in part I and modeling results in part II.

\section{Ionization Stage}

- In chapter 3, the different experimental setups are described: the helicon reactor, the PEGASES thruster prototype I, the vacuum chamber for the thruster, and the matchbox for the thruster.

- In chapter 4, the ionization stage in the first PEGASES prototype is investigated, with emphasis on the energy coupling, the positive ion flux, the mass efficiency, and the electron temperature profile.

\section{Magnetic Electron Filtering Stage}

- In chapter 5 , the experimental aspect of the magnetic electron filtering is investigated with Langmuir-type probes, a retarding field energy analyzer, and electronegativity measurements. First, in the helicon reactor in which ion-ion plasmas were originally obtained, then, in the PEGASES thruster prototype I, in which ion-ion plasmas could only be obtained after design improvements.

- In chapter 6 , the fluid modeling of the magnetic electron filtering, the result of the collaboration with UC Berkeley, is presented. It consists of numerical and analytical solutions to the fluid equations of the model. The role of electron and positive ion axial losses (along the magnetic field lines) is stressed. The scaling of the main outputs (negative ion flux and thruster radius) is described as a function of the main parameters.

\section{Extraction and Acceleration Stage}

- In chapter 7, the structure of sheaths in ion-ion plasmas is investigated. The problem is first treated with basic fluid models, corresponding to two extreme cases of ionion plasmas. The existence of a Bohm criterion for ion-ion plasmas is then shown with PIC simulations and a kinetic model. With this Bohm criterion fulfilled in the kinetic model, two cases are studied: high voltage sheaths corresponding to a biased grid, and low voltage sheaths corresponding to the potential structure with dielectric walls (floating potential). It will be shown that the classical scaling law of the floating potential in the electron-positive ion case is woefully wrong for ion-ion plasmas.

In chapter 8, the different conclusions from the work presented in this thesis are summarized, as well as a brief overview of the future of the PEGASES thruster project. 


\section{Chapter 2}

\section{Models and Diagnostics for Plasmas}

\section{Contents}

2.1 Models for Plasmas .................. 17

2.1 Notations and Units . . . . . . . . . . . . . . . . . . 17

2.1.2 The Various Models . . . . . . . . . . . . . . . . . . . 18

2.1.3 Maxwellian Species . . . . . . . . . . . . . . . . . 22

2.1.4 Boltzmann Relation . . . . . . . . . . . . . . . 22

2.2 Diagnostics . . . . . . . . . . . . . . 22

2.2 .1 Langmuir Probes . . . . . . . . . . . . . . . . . . . . . 23

2.2.2 Voltage and Current Probe . . . . . . . . . . . . . . 29

2.2.3 Retarding Field Energy Analyzer . . . . . . . . . . . . . . . . 29

2.2 .4 Instruments . . . . . . . . . . . . . . . . . 31

2.2 .5 Computer Programs . . . . . . . . . . . . . . . . . . . . . . . . 33

2.3 Electronegativity Measurement Techniques . . . . . . . . . 34

2.3.1 Review of Techniques . . . . . . . . . . . . . . . 34

2.3 .2 Electrostatic Probe . . . . . . . . . . . . . . 36

Whether for experiments or modeling, various plasma models are needed to understand the plasma equilibrium. These models, as well as notations and units, are first described. The diagnostics used to characterize the plasma equilibrium in experiments are then presented. These are Langmuir-type probes, voltage and current probes, retarding field energy analyzer, and the various instruments and computer programs. Finally, electronegativity measurement techniques are presented, with emphasis on the one used in this thesis, the electrostatic probe.

\subsection{Models for Plasmas}

\subsubsection{Notations and Units}

\section{General Notations}

As plasmas are modeled in this thesis, three species are involved. For any variable related to one of these species, a subscript is used: (e) electrons, $(+)$ positive ions, and (-) negative 
ions.

The charge of a particle is noted $q$ with the elementary (electron) charge $e$. The density $n$ is given in $\mathrm{m}^{-3}$, the velocity $v$ in $\mathrm{ms}^{-1}$, the flux $\Gamma$ in $\mathrm{m}^{-2} \mathrm{~s}^{-1}$, the mass $m$ in $\mathrm{kg}$.. The potential is written as $\varphi$ in $\mathrm{V}$, the electric field as $E$ in $\mathrm{Vm}^{-1}$ and the magnetic field as $B$ in $\mathrm{G}$. For the temperature, the choice is made to use volts, with the following relation between volts and kelvins

$$
T(K)=\frac{e}{k_{B}} T(V),
$$

with $k_{B}$ the Boltzmann constant. It can be calculated that $1 \mathrm{~V}$ is equivalent to $1.16 \times 10^{4} \mathrm{~K}$.

The vacuum permittivity is noted as $\varepsilon_{0}$.

The ionization fraction, electron density divided by the neutral density, is written as $n_{e} / n_{g}$. The ratio of negative ion density to electron density is noted as $\alpha=n_{-} / n_{e}$. The ratio of electron to negative ion temperature is $\gamma=T_{e} / T_{-}$. The edge-to-center density ratio is noted $h$, and represents the drop in the density from the center of the plasma to the sheath edge.

\section{Fluid Notations}

The reaction rates are noted $K: K_{\text {iz }}$ for ionization, $K_{\text {att }}$ for attachment, $K_{\text {det }}$ for detachment, and $K_{\text {rec }}$ for ion-ion recombination. The neutral density is written as $n_{g}$. Similarly, the frequencies are noted $\nu$, with $\nu_{\mathrm{iz}}=K_{\mathrm{iz}} n_{g}$ the ionization frequency for instance. In the case of collisions with neutrals, the momentum transfer rate is noted $K_{m}$ and the frequency $\nu$. In the case of electrons, $\nu_{e}=K_{\mathrm{m}, \mathrm{e}} n_{g}$.

The plasma frequency of a species is noted $\omega_{p}$, with the following expression

$$
\omega_{p}=\sqrt{\frac{q^{2} n}{\varepsilon_{0} m}} .
$$

As to the cyclotron frequency, it is noted $\omega_{c}$

$$
\omega_{c}=\frac{q B}{m} .
$$

\subsubsection{The Various Models}

A plasma is a collection of charged particles (electrons and ions) and neutral particles (background neutrals and excited neutrals). Following the position and velocity of each and every particle as a function of time for all possible interactions (electric force, collisions, etc.) is an accurate description and its principle is similar to the N-body problem where the position of all particles is followed as a function of time with Newton's laws. However, such a problem cannot be solved, whether analytically or numerically, due to the sheer amount of information needed: a six-dimensional vector (three for position and three for velocity) as a function of time (continuous if analytical, with a small enough time step if numerical) for all particles. For the example of a neutral gas with a pressure of $10 \mathrm{~m}$ Torr in a volume of $1 \mathrm{~cm}^{3}$ (very low pressure in a very small volume), there are $3.3 \times 10^{14}$ particles to account for, leading to $6.6 \times 10^{14}$ particles for full ionization (each neutral yields an ion and an electron). Moreover, a complete description of all interactions between the particles is not feasible.

However, it is possible to use macro-particles to describe the plasma equilibrium, with each macro-particle representing a large number of real particles. These simulations are 
called Particle-In-Cell (PIC) simulations [28]. The cells are defined by the mesh points and are used for the calculation of the fields on the mesh points. The macro-particles do not interact with one another with the Coulomb force, but through the fields. The collisions between macro-particles can be taken into account through Monte-Carlo simulations. PIC simulations can be used in one, two or three dimensions, but the computations, even in one dimension, are quite time-consuming. For example, the PIC simulations presented in chapter 7 were more than a week long for $2 \times 10^{6}$ macro-particles and an average of 2000 cells.

\section{Kinetic description}

The first order of approximation is called the kinetic description. Instead of following individual particles, a distribution function for each species is considered with the position, velocity and time as function parameters: $f(\mathbf{r}, \mathbf{v}, t)$. For any given $\left(\mathbf{r}_{\mathbf{0}}, \mathbf{v}_{\mathbf{0}}, t_{0}\right)$, this function returns the number of particles at the position $\mathbf{r}=\mathbf{r}_{\mathbf{0}}$ with the velocity $\mathbf{v}=\mathbf{v}_{\mathbf{0}}$ for $t=t_{0}$. Using the continuity equation for the distribution function over $d t$, the Boltzmann equation can be obtained

$$
\frac{\partial f}{\partial t}+\mathbf{v} \cdot \nabla_{\mathbf{r}} f+\frac{\mathbf{F}}{m} \cdot \nabla_{\mathbf{v}} f=\left.\frac{\partial f}{\partial t}\right|_{c},
$$

with $\mathbf{F}$ the sum of the forces applied on the particle. The right hand side term is called the collision term and accounts for elastic and inelastic collisional processes, among which are particle creation and loss. When it is assumed to be zero, the Boltzmann equation becomes the Vlasov equation

$$
\frac{\partial f}{\partial t}+\mathbf{v} \cdot \nabla_{\mathbf{r}} f+\frac{\mathbf{F}}{m} \cdot \nabla_{\mathbf{v}} f=0 .
$$

For further simplification of equations (2.4) and (2.5), the number of dimensions can be restricted to one or two. The Vlasov equation in one dimension is written as

$$
\frac{\partial f}{\partial t}+v \frac{\partial f}{\partial x}+\frac{F}{m} \frac{\partial f}{\partial v}=0
$$

This equation is used in chapter 7 for the modeling of sheaths in ion-ion plasmas. For a characteristic sheath size smaller than the ion mean free path (distance between two collisions for ions), the collisionless assumption is justified.

\section{Fluid description}

The next order of approximation is the fluid description. Here, all quantities are the result of an integration over the velocity components of the distribution functions. A moment of order $i$ of the distribution function $f$ is defined as the integration of the product of this function and its argument (here velocity) to the $i^{\text {th }}$ power,

$$
M_{i}(f)(\mathbf{r}, t)=\iiint_{\mathbb{R}^{3}} \mathbf{v}^{i} f(\mathbf{r}, \mathbf{v}, t) d \mathbf{v} .
$$

By definition of the distribution function, the first moment $i=0$ is the density at $(\mathbf{r}, t)$, and the moment $i=1$ is the average velocity at $(\mathbf{r}, t)$. To obtain relations between the variables, the Boltzmann equation (2.4) is integrated using the definition of moments (2.7). 
The equations are called continuity equation for $i=0$ (direct integration), momentum conservation for $i=1$ (integration of $m v f$ ) and energy conservation for $i=2$ (integration of $\left.\frac{1}{2} m v^{2} f\right)$. The knowledge of all the moments of a function, i.e. $i \in \mathbb{N}$, is equivalent to the knowledge of the function. Since solving all moments of the Boltzmann equation is impossible, only a few are considered (two in most cases, sometimes three). The issue with this limited approach resides in the fact that one variable needed to solve the moment of order $n$ is solution of the moment of order $n+1$. For example the temperature, solution of the energy conservation, is needed to solve the momentum conservation. Assumptions are thus needed to close the system and obtain as many variables as equations. When solving two moments of the fluid equations, the particle temperatures are usually determined by considering an additional equation, such as the power balance (described in equations (2.14) through (2.16)). In the numerical integration of the fluid model presented in chapter 6 , a constant electron temperature is determined by the boundary conditions.

Fluid models presented in chapters 6 and 7 of this thesis are one-dimensional $(\mathbf{r}=x)$ and steady-state $\left(\frac{\partial}{d t} \cdot=0\right)$.

The general expression of the continuity equation is

$$
\nabla(n v)=G-L
$$

with $G$ the creation term and $L$ the loss term, both of which come from the collision term in the Boltzmann equation (2.4). Ionization is an example of creation term and recombination an example of loss term. The continuity equation is thus the evolution of the flux as particles are created or lost.

An approximation of the momentum conservation equation is

$$
\underbrace{e T \nabla n}_{\text {pressure }} \pm \underbrace{e n \nabla \varphi}_{\mathrm{E} \text { force }}+\underbrace{m n v \nabla v}_{\text {inertia }}+\underbrace{m \nu n v+m v(G-L)}_{\text {collisions }}=0
$$

with \pm being + for positively charged particles or - for negatively charged. The pressure term uses the assumption of constant temperature as the full expression is $e \nabla(T n)$. The electric force term comes from the fluid electric force $\mp e n E$ and the definition between potential and electric force $E=-\frac{\partial \varphi}{\partial x}$. The inertia term is the remnant part of the total derivative

$$
\frac{d}{d t} \cdot=\left(\frac{\partial}{\partial t}+\frac{d x}{d t} \frac{\partial}{\partial x}\right) \cdot=\left(\frac{\partial}{\partial t}+v \frac{\partial}{\partial x}\right) \cdot
$$

There are two kinds of collisions taken into account. The first corresponds to collisions of the particle with background neutrals, and the second to momentum losses when particles are created or lost. This second term is usually combined with the inertia term by using equation (2.8) and yields the general expression of the momentum conservation equation

$$
\underbrace{e T \nabla n}_{\text {pressure }} \pm \underbrace{e n \nabla \varphi}_{\mathrm{E} \text { force }}+\underbrace{m \nabla\left(n v^{2}\right)}_{\text {inertia }}+\underbrace{m \nu n v}_{\text {collisions }}=0
$$

It should be noted that $m \nabla\left(n v^{2}\right)$ is referred to as the inertia term although it is the combination of the inertia term from the total derivative and the collision term from creation and losses. 


\section{Global description}

As the variables were integrated over the velocity from the kinetic to the fluid description, they are now integrated over the position to obtain the global description. Such models are often called 0D models as the dependence on the position has been removed and variables characterize the plasma in its entirety. Their goal is to relate the plasma and what is exterior to it: how the power is coupled, what happens at the walls, etc.

The plasmas considered are always enclosed in a volume, defined by surrounding walls. As a plasma is created, the electrons and ions diffuse, to be lost either in the volume (recombination for positive ions for instance) or at the walls (recombination). Due to the mass ratio, the electron diffusion is faster than that of the ions: more electrons are collected at the walls, which becomes negatively charged compared to the plasma potential. In turn, positive ions are accelerated towards the wall while electrons are confined to the center of the discharge (electropositive case). Since a plasma should be quasi-neutral, the potential difference is localized near the walls, in a region called the sheath. The complete structure is thus plasma/sheath/wall.

With a radio-frequency excitation of frequency $\omega$, the ions cannot respond to the electromagnetic field as $\omega$ is higher than the ion plasma frequency. The plasma frequency $\omega_{p}$ for a particle is defined as the frequency with which it responds to a perturbation, and its expression is

$$
\omega_{p}=\sqrt{\frac{q^{2} n}{\varepsilon_{0} m}} .
$$

The scaling between the frequencies is

$$
\omega_{p+}<\omega<\omega_{p e}
$$

Therefore, the power is only coupled to the electrons, with a simplified power balance written as

$$
\frac{d}{d t}\left(\frac{3}{2} n_{e} e T_{e} \mathcal{V}_{T}\right)=P_{\text {abs }}-P_{\text {loss }}
$$

with $P_{\text {abs }}$ the electrical power absorbed by the plasma, $P_{\text {loss }}$ the electron power lost in the plasma and at the walls, $\mathcal{V}_{T}$ the total volume of the plasma, and a $\frac{3}{2}$ factor as the plasma considered is three dimensional. With $\mathcal{E}_{T}$ the total energy loss per electron-ion pair created (dependent on the electron temperature), the power lost in the plasma can be written as

$$
P_{\text {loss }}=\Gamma_{e} \frac{\mathcal{A}_{\text {wall }}}{\mathcal{V}_{T}} \mathcal{E}_{T}\left(T_{e}\right),
$$

with $\Gamma_{e}$ the electron flux at the walls, and $\mathcal{A}_{\text {wall }}$ the area of the walls. In a steady-state regime, the absorbed and lost power compensate one another

$$
P_{\text {abs }}=P_{\text {loss }}
$$

As the electron flux is proportional to the electron density, equations (2.15) and (2.16) mean that for a relatively constant electron temperature, the electron density is proportional to the applied power. It should be noted that the power balance presented here was simplified for the sake of clarity. 


\subsubsection{Maxwellian Species}

When a species reaches a thermodynamic equilibrium, its velocity distribution function can be expressed as a function of its temperature

$$
f(v)=A \exp -\frac{m v^{2}}{2 e T}
$$

with $A$ a normalization factor, usually determined by the density with $n=\int_{\mathbb{R}} f(v) d v$. It can be seen that this expression is in fact a balance between kinetic energy $\left(\frac{1}{2} m v^{2}\right)$ and thermal energy $(e T)$.

Although the considered plasmas are never in thermodynamic equilibrium, it is possible for some species to be in such an equilibrium on their own. For instance electrons are often assumed to be Maxwellian in global models and simple fluid models.

The kinetic model in chapter 7 uses Maxwellian-based distribution functions as assumptions to solve the Vlasov equation (2.6).

\subsubsection{Boltzmann Relation}

The Boltzmann relation is derived from equation (2.11) for the electrons. They are assumed to be massless, the inertia term is dropped, and collisionless. This equation becomes

$$
e T_{e} \nabla n_{e}=e n_{e} \nabla \varphi
$$

where only two terms are kept, pressure and electric force. Simplifying it yields

$$
\frac{\nabla n_{e}}{n_{e}}=\frac{\nabla \varphi}{T_{e}}
$$

For $x=0$, which usually corresponds to the center of the plasma, the electron density is set to $n_{e 0}$ and the potential to zero. Equation (2.19) can be integrated between 0 and $x$

$$
\int_{0}^{x} d\left(\ln n_{e}\right)=\int_{0}^{x} \frac{d \varphi}{T_{e}}
$$

which gives the expression of the Boltzmann relation

$$
n_{e}(x)=n_{e 0} e^{\varphi(x) / T_{e}}
$$

With the approximation in (2.18), the electron density is only a function of the potential.

\subsection{Diagnostics}

Two kinds of electrical diagnostics were used to characterize the plasma: Langmuir-type probes and a retarding field analyzer. These require additional instruments and computer programs for repetitive and/or fast measurements. 


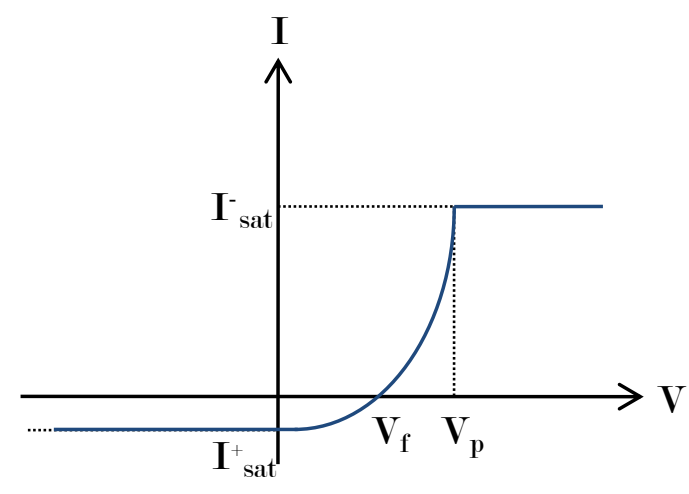

(a)

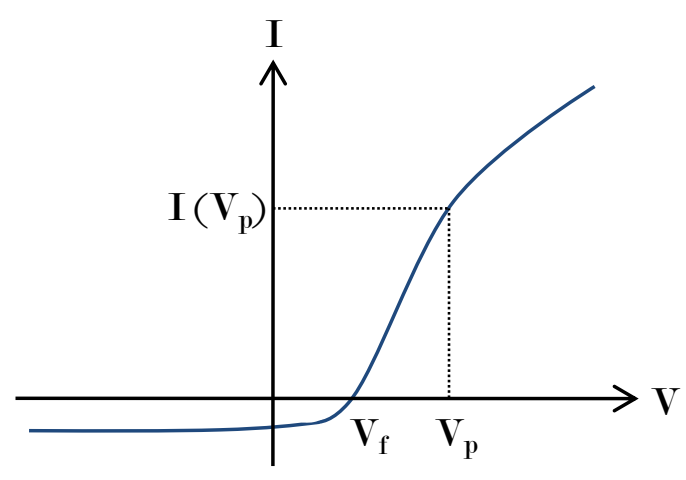

(b)

Figure 2.1: Sheath-free I-V characteristic (a) and actual I-V characteristic (b) for plasmas where the electrons dominate the equilibrium. The current from negative charges (electrons and negative ions) is arbitrarily chosen as positive.

\subsubsection{Langmuir Probes}

\section{Principle}

The idea of Langmuir-type probes consists of placing a conductor in the plasma and looking at the collected current for a given bias or range of bias. The curve of the collected current as a function of polarization voltage is called an I-V characteristic, with the arbitrary choice of plotting the electron current as positive, and the positive ion current as negative as a consequence.

The plasma potential $V_{p}$ is the potential at which the plasma sets itself in regards to the surrounding walls in order to confine electrons when these dominate the plasma equilibrium. As a result, all species, regardless of their velocities, are collected by a conductor biased to this potential. The floating potential $V_{f}$ is the potential for which a biased probe would collect a net current of zero. Although the net current is zero, positive (ions) and negative (electrons, negative ions) charges are collected.

In a case where temperatures are negligible for all species, an I-V characteristic would be a step function centered around $V_{p}$ with $I\left(V<V_{p}\right)=I_{\text {sat }}^{+}$and $I\left(V>V_{p}\right)=I_{\text {sat }}^{-}$. In an electron dominated plasma, however, the electron temperature is too high $(\sim 3 \mathrm{eV})$ to be neglected while the ion temperatures are low. This means that an important part of the electrons have a high velocity, with the ability to overcome potential barriers. With the assumption of a Boltzmann equilibrium (see equation (2.21) in section 2.1.4), the expression of the density of electrons for a given voltage applied to a conductor is

$$
n_{e}(V)=n_{e 0} e^{\left(V-V_{p}\right) / T_{e}} .
$$

Assuming a thermal flux for the electrons, the current is

$$
I_{e}=I_{e 0} e^{\left(V-V_{p}\right) / T_{e}} \quad \text { with } \quad I_{e 0}=e \mathcal{A} n_{e 0} \frac{\bar{v}}{4}=e \mathcal{A} n_{e 0} \sqrt{\frac{e T_{e}}{2 \pi m_{e}}},
$$

with $\mathcal{A}$ the area of collection. As $V$ is decreased from $V_{p}$, the electron current is decreasing exponentially. The resulting I-V characteristic can be seen in figure 2.1a. Increasingly, as soon as $V$ reaches $V_{p}$, all electrons are collected which is the reason why there is a 
saturation for $V>V_{p}$. As $V$ is decreased from $V_{p}$, more and more electrons are repelled, until a certain voltage where all electrons are reflected and the collected current is a pure positive ion current. During this phase, the floating potential is reached when the potential difference $\left(V_{f}-V_{p}\right)$ reduces the electron current to the positive ion current. For smaller values of bias, the current saturates to the positive ion current.

The previous description does not take sheaths into account for the saturation currents. As the bias $\left|V-V_{p}\right|$ is increased, either toward the negative or positive current, a sheath is formed between the plasma and the conductor. The size of this sheath increases with the potential difference $\left|V-V_{p}\right|$. As a consequence, the volume of the sheath region increases, which means that the collection surface (sheath surface) is increased with $\mid V-$ $V_{p} \mid$. Therefore, there is no saturation for $V>V_{p}$ or $V<V_{p}$ with all electrons reflected, but an increase of absolute collected current. Although the scaling is the same for electrons and ions, this increase is more significant in the case of electron collection because of the temperature difference between electrons and ions. The resulting I-V characteristic can be seen in figure $2.1 \mathrm{~b}$.

An I-V characteristic can be used to obtain different plasma parameters. The first parameter is the floating potential as it can be easily determined from an I-V characteristic: $I\left(V_{f}\right)=0$. The second parameter is the plasma potential. For $V<V_{p}$ the current follows an exponential (repelled electrons) and its curvature is thus positive. For $V>V_{p}$, it is slowly increasing as a result of sheath expansion with a negative curvature. Therefore, the point $I\left(V_{p}\right)$ is an inflection point and can be determined by calculating the second derivative with $\frac{d^{2} I\left(V_{p}\right)}{d V^{2}}=0$. The third parameter is the ion current $I_{i}$. Although there is no flat saturation current because of sheath expansion, the ion current can be determined using the Orbital Motion Limited theory [29] for a cylindrical collector which gives $I_{i}(V) \propto$ $\sqrt{V-V_{p}}+$ const with the proportional factor depending on the probe dimensions. The fourth parameter is the electron temperature $T_{e}$ that can be obtained with the assumption of Boltzmann electrons. Taking the logarithm of the electron current in (2.23) gives

$$
\ln I_{e}=\ln I_{e 0}+\frac{V-V_{p}}{T_{e}} .
$$

The electron temperature can thus be determined from the slope of $\ln I_{e}$ as a function of $V$. It should be noted that if the ion current is non-negligible compared to the electron current, the electron temperature is obtained from the slope of $\ln \left(I-I_{i}\right)$ versus $V$. The last parameter is the electron density, determined from the current at $V_{p}$ and equation (2.23) where the expression of the average velocity $\bar{v}$ is a function of the electron temperature. Since there is no sheath at $V=V_{p}$, the area of collection $A$ is the probe area.

The situation for an ion-ion plasma differs from the electron dominated one as both species, negative and positive, have comparable masses and temperatures, usually much lower than the electron temperature in a classical electron-ion plasma. Because of symmetry, the floating potential is zero and the saturation currents are the same: the I-V characteristic is symmetrical around the value $V=V_{f}=0$. I-V characteristics, therefore, can be used to determine whether a plasma is electron dominated (asymmetric) or an ion-ion plasma (symmetric), as it will be discussed in chapter 5 .

\section{Radio-frequency Discharges}

For a radio-frequency excited plasma, the plasma potential oscillates at the same frequency as the excitation. Harmonics, mainly the second one, can also be found. This means that 


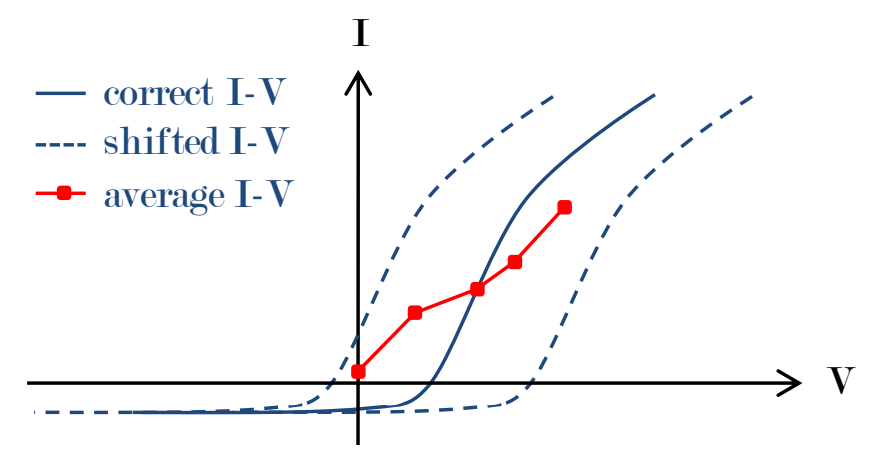

Figure 2.2: $I-V$ characteristics with a radio-frequency excitation with the correct one (blue), two shifted characteristics at maximum radio-frequency amplitudes (dashed blue curve), and the average one obtained with a probe for a few points (red).

the I-V characteristic of such a plasma oscillates around the correct I-V characteristic, as can be seen in figure 2.2 [30]. The correct average of the I-V characteristic is shown in blue: parameters obtained with it are the plasma parameters. As the plasma oscillates at the excitation frequency, the I-V characteristic is shifted from the correct average between the minimum excitation voltage (left dashed blue curve) and the maximum excitation voltage (right dashed blue curve). A probe measuring the I-V characteristic with a time constant bigger than the period of the excitation would obtain an average of all the shifted curves (red curve). Parameters obtained from this curve are meaningless. Therefore, a method to obtain the correct average is needed.

To do so, inductances are placed in series of the probe collector which resonate with the parallel capacitance at a frequency equal to the excitation frequency $(13.56 \mathrm{MHz})$ and its second harmonic $(27.12 \mathrm{MHz})$. These inductances, moreover, are placed as close as possible to the collector to optimize the filtering. As a result, only the direct-current component of the current is collected, giving the correct averaged I-V characteristic.

\section{Magnetic Field}

An I-V characteristic is obtained by collecting currents at different bias values. With a strong enough magnetic field, the Larmor radius of the plasma species can become comparable, or even smaller, than the length of the collector. As electrons are much lighter than ions, they are the first to be magnetized. When magnetized, not all electrons entering the sheath around the collector are collected, resulting in an underestimation of the electron current. The electron current $I_{e}$ being the main part of an I-V characteristic, the latter is decreased yielding to incorrect plasma parameter calculations.

Although models were developed to take the magnetic field into account [31, 32], the small dimension of the probe used for measurements allows us to assume that it is smaller than the Larmor radius (a few millimeters for $T_{e}=3 \mathrm{~V}$ and $B_{0}=250 \mathrm{G}$ ). Moreover, the direction of the filament compared to the magnetic field lines plays a role in how the magnetic field affects the electron current collection. With the filament parallel to the magnetic field lines, electrons tend to circle around the filament instead of being collected. The underestimation of the electron current is strong in this situation. With the filament perpendicular to the magnetic field lines, the circling motion of the electrons does not affect their collection. The effect of the magnetic field is small in this situation. All 


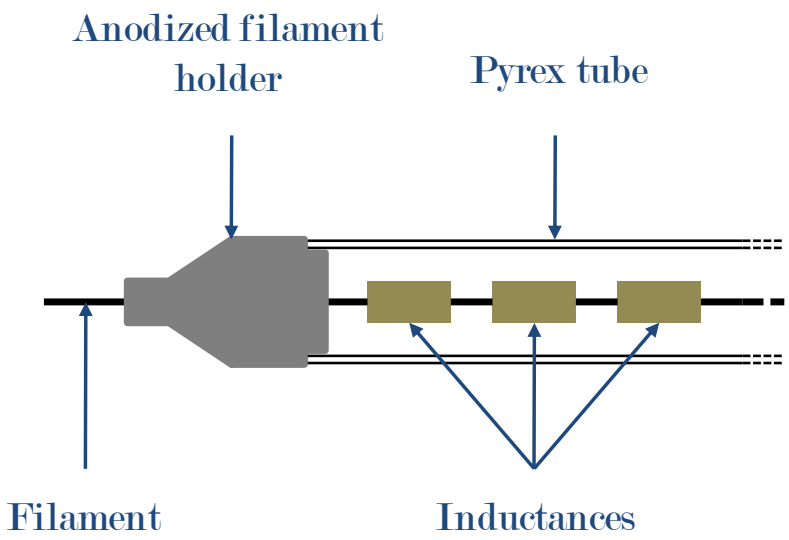

(a)

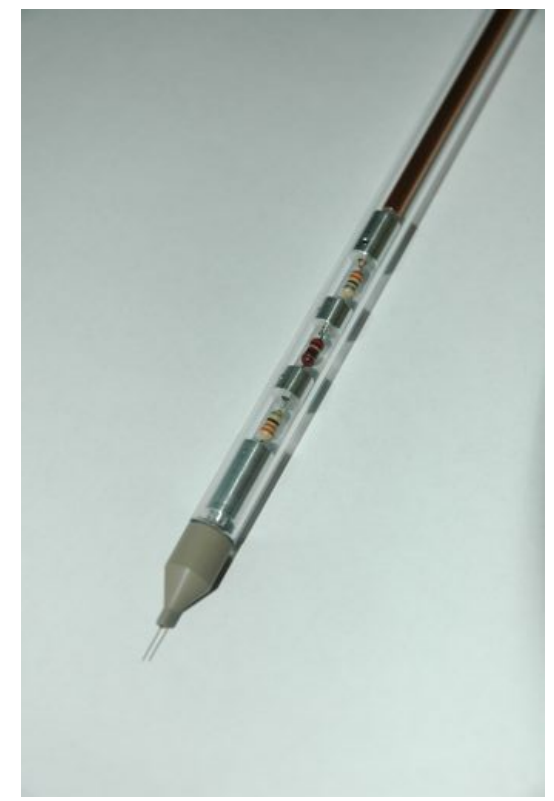

(b)

Figure 2.3: Schematics (a) of the filament probe with three inductances in series and photo (b) of the filament probe.

measurements done with filament probes in either the helicon reactor or the PEGASES thruster prototype were done perpendicularly to the magnetic field lines. This and the value of the Larmor radius justify the assumption of an unchanged electron current even in the presence of the magnetic field.

\section{Filament Probe}

The probe used for I-V characteristic measurements is a filament probe (see figures 2.3ab). The filament itself is either a platinum or a tungsten one. The melting point of tungsten $\left(3422^{\circ} \mathrm{C}\right)$ is higher than that of platinum $\left(1768^{\circ} \mathrm{C}\right)$. As a probe is sometimes biased above the plasma potential, the accelerated electrons that are collected heat the filament up, making tungsten safer to use than platinum. However, a plasma of sulfur hexafluoride reacts with tungsten resulting in the destruction (etching) of the filament, which does not occur with platinum. The filament holder is anodized to minimize the interaction with the plasma and the current collection. With a worn out filament holder, undesirable electric arcs can occur between the filament and its holder while the filament is biased. The coaxial cable links the last inductance to the connection on the panel. The inductances and the coaxial cable are protected from the plasma by a pyrex tube.

A choice of two inductances with a resonant frequency of $13.56 \mathrm{MHz}$ and a third one with $27.12 \mathrm{MHz}$ was made to ensure proper filtering of the radio-frequencies [21]. Each inductance was tested with a network analyzer to determine its correct resonant frequency as the commercial inductances used have a relative error of $5 \%$, leading to a range from $12 \mathrm{MHz}$ to $15 \mathrm{MHz}$ for $13.56 \mathrm{MHz}$ for instance.

For all measurements, whether in the helicon reactor or on the thruster, the probe was mounted on a translator allowing radial measurements over $30 \mathrm{~cm}$. The probe can be moved without breaking the vacuum of the chamber. The reference for distances is the middle of the filament. 


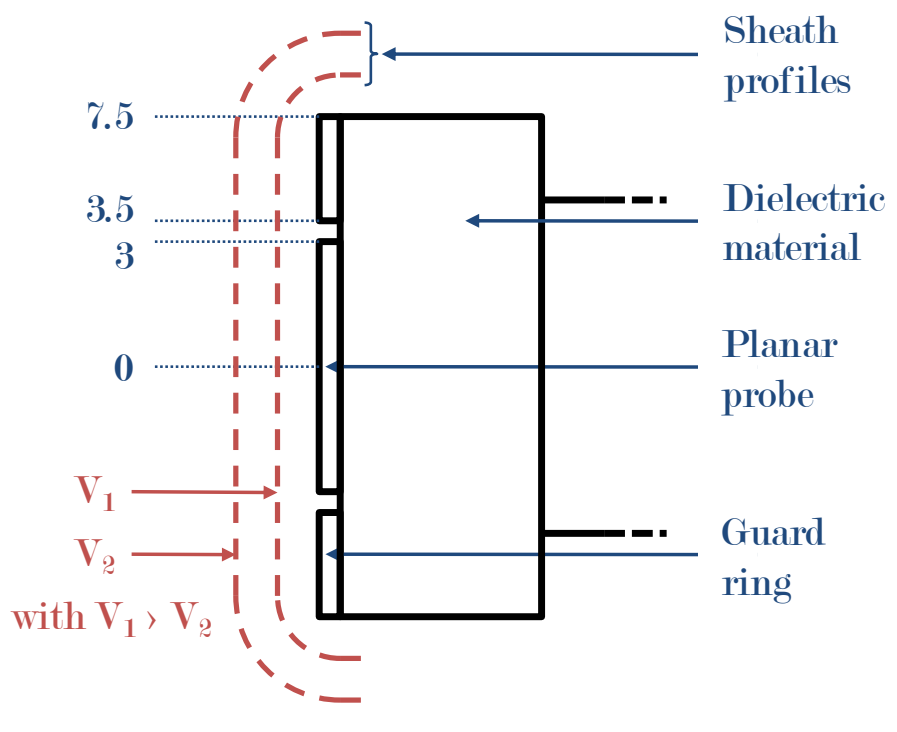

(a)

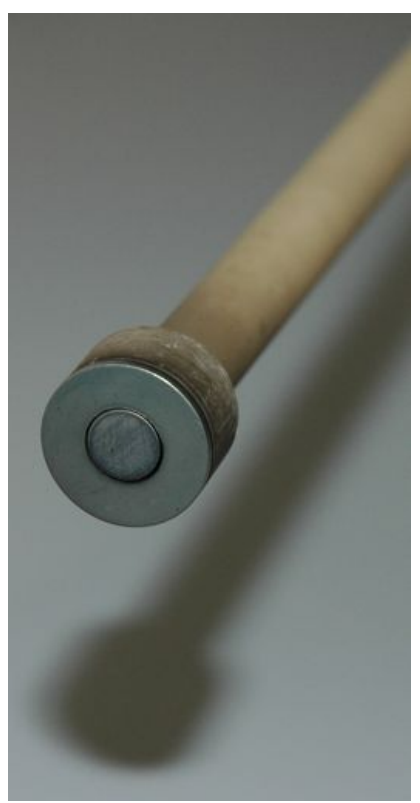

(b)

Figure 2.4: Schematics (a) of the planar probe with distances in $\mathrm{mm}$ and sheath profiles for $V_{1}$ and $V_{2}$ with $V_{1}>V_{2}$ (negative polarizations); photo (b) of the planar probe.

\section{Planar Probe}

The ion current $I_{i}$ can be calculated from the I-V characteristic using the Orbital Motion Limited theory with a filament probe where the sheath expansion is taken into account. As this theory assumes that the particles are collisionless, it quickly becomes invalid as the sheath size increases. However, the geometry of the probe can be optimized for ion current measurements limiting the sheath expansion: with a planar probe, the collection area of the sheath varies little as the potential difference $\left|V-V_{p}\right|$ is increased. This can be further optimized with a guarded planar probe: the probe is surrounded by a conductor polarized to the same potential. The sheath in front of the probe is thus parallel to the probe: biasing the probe negatively from $V_{1}$ to $V_{2}$ with $V_{1}>V_{2}$ increases the volume of the sheath region, but does not change the collection area, as can be seen in figure $2.4 \mathrm{a}$ with two sheath profiles for two biases. The guarded planar probe used in the experiments is shown in figure 2.4b. The planar probe, diameter $6 \mathrm{~mm}$, is separated from the guard ring, diameter $15 \mathrm{~mm}$, by a $0.5 \mathrm{~mm}$ gap. The collection area of the planar probe is $\mathcal{A}_{p}=28.3 \mathrm{~mm}^{2}$ which gives the following relationship between the collected current $I_{c}$ in $\mathrm{A}$ and the current density $J_{c}$ in $\mathrm{Acm}^{-2}$

$$
J_{c}=\frac{I_{c}}{28.3 \times 10^{-2}}
$$

As the electron current is usually bigger than the ion current, a large collection area results in high currents. To avoid this, planar probes are only used in the ion part of the I-V characteristic. 


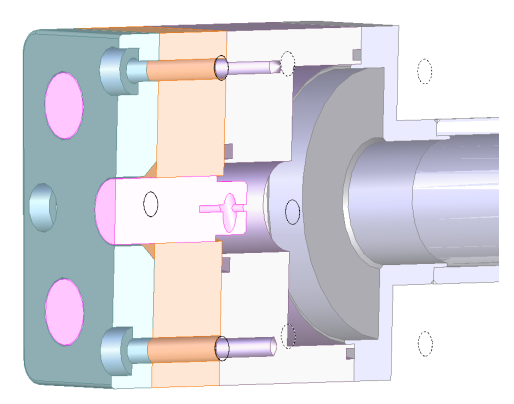

(a)

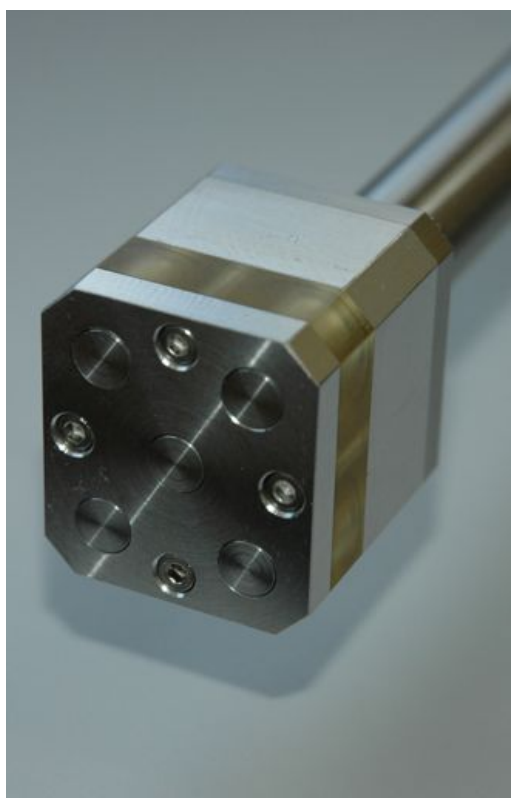

(b)

Figure 2.5: Schematics (a) of the multi-planar probe and photo (b) of the multiplanar probe. A small interstice separates each probe from the metal casing, while a dielectric material (polysulfone) separates the conducting surfaces of the probe and the main body supporting the probe.

\section{Multi-Planar Probe}

Given the cylindrical geometry of the first PEGASES prototype, the ion current exiting through the extractor tubes is not uniform. The natural expansion of a plasma in a cylinder is radial while the direction of the extractor tube is the same as the cylinder at the center only and makes an angle otherwise (the further away from the center, the bigger the angle). In order to investigate the ion current over the area of the extractor tubes, a multi-planar probe was designed.

This probe, shown in figures $2.5 \mathrm{a}-\mathrm{b}$, consists of a combination of five planar probes incased in a conducting surface that can act in the same way as a guard ring. Each probe is connected via a coaxial cable to a specific pin of a IEEE-488 connector (maximum of 36 pins), with the addition of a coaxial cable linking a pin of this connector and the body, acting as a guard ring, where the probes are encased. The switching behavior control and the analog-to-digital converters are placed in a box which is the intermediary between the probes and the computer. The direct-current generator used to bias the probes is connected to this box. Although a simultaneous reading of all the probes would be ideal, it is not possible. Instead, each probe is read one after the other, with a total read time for five probes of the order of $100 \mathrm{~ms}$. In case of instabilities, this characteristic read time could alter the measurements. A program in LabView, written by Philippe Auvray, controls the box and collects the measurements. These are shown in a graphical form where the color intensity (from white to dark blue) gives the value of current compared to the other probes. 


\subsubsection{Voltage and Current Probe}

\section{Coaxial Probe}

During his $\mathrm{PhD}$, Sebastien Dine designed a probe capable of measuring both voltage and current at high power on a coaxial cable connection for a wide range of frequencies [33]. As both are measured simultaneously, the phase difference can be deduced, with a limitation for high frequencies.

The voltage is measured by capacitive coupling between the center of the coaxial cable and a conic conductor placed near it and shielded from perturbations as it is encased in the outer conductor of the coaxial cable. The conic shape was chosen for a better frequency response.

The method to measure the current is similar to an inductive probe, often called Bdot probe. A variable current in a conductor creates a variable magnetic field, whose flux is collected over an area, resulting in a potential difference (Maxwell equations). The perturbation of the measurement can be modeled as an additional inductance around $1 \mathrm{nH}$ in the circuit, and is negligible compared to other inductances, e.g. tens of $\mathrm{nHm}^{-1}$ for the coaxial cable.

To limit the coupling between the two measurements, the devices are set apart. This distance is the reason for the limitation of phase measurements between the voltage and the current: for high frequencies, the distance is no longer negligible compared to the wavelength of the voltage and current. An intrinsic phase difference between the voltage and current is introduced and depends on the frequency.

\section{High Voltage Probe}

In order to measure the voltage across the antenna, a point-to-point high voltage probe is needed. The high voltage probe used was a Lecroy PPE20kV, with a maximum measured voltage of $20 \mathrm{kV}$. The upper end of the bandwidth is $100 \mathrm{MHz}$, meaning that measurements of the first two harmonics $(13.56 \mathrm{MHz}$ and $27.12 \mathrm{MHz}$ ) are possible. The probe is connected to an oscilloscope to read the measurements.

\subsubsection{Retarding Field Energy Analyzer}

A retarding field energy analyzer, commonly called RFEA, uses an adjustable potential barrier to determine the kinetic energy of the collected particles. A minimum of two grids is thus necessary to create the potential barrier [34], but designs with four grids were developed [35]. For a potential barrier $V_{b}$, the minimum velocity $v_{b}$ required to overcome this barrier is given by the conservation of energy

$$
\frac{1}{2} m v_{b}^{2}=e V_{b}
$$

which means that the collected current with the distribution function $f(v)$ for a given polarization $V_{b}$ is

$$
I\left(V_{b}\right)=\int_{\sqrt{2 e V_{b} / m}}^{+\infty} f(v) d v .
$$

For each value of bias on the probe, the collected current corresponds to all particles with a kinetic energy higher that the potential difference, and decreases as the bias is increased (figure 2.6a). The shape of the distribution function can be obtained by taking 


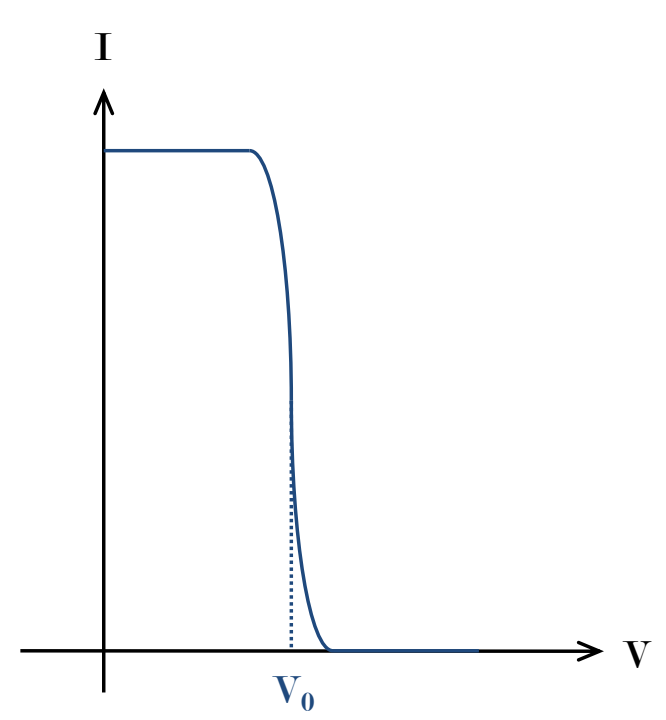

(a)

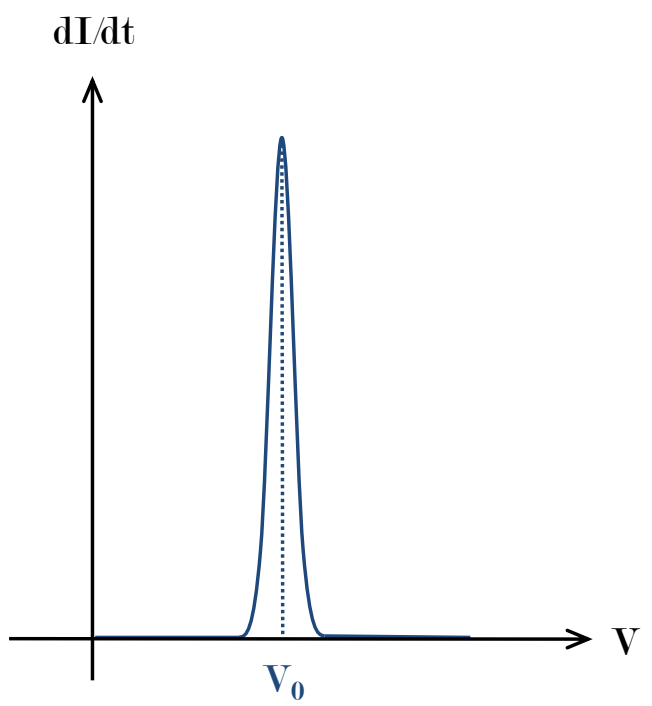

(b)

Figure 2.6: Collected current (a) and derivative of the collected current (b) for an RFEA probe. $V_{0}$ corresponds to the center of the symmetrical distribution function.

the derivative of the collected current (figure 2.6b). In figure 2.6, the distribution function of the particles presents only one peak centered around $V=V_{0}$, which means that the particles are mono-energetic. In the case of two populations with two different mean energies, the collected current would show two steps.

In the two-grid design, the first grid $\left(\mathrm{G}_{0}\right)$ is grounded and provides an equipotential. The second grid, called the reject grid $(\mathrm{R})$, is biased to a value ensuring that the oppositely charged species is completely reflected. The collector is biased with the retarding potential, which is swept from zero (ground) to a value where the collected current reaches zero (energy higher than the maximum energy of the particles). As particles are collected, secondary electrons are emitted. The current is then overestimated in the case of positive particles, and underestimated for negative particles. To remedy this problem, two additional grids are placed between the reject grid and the collector. The third grid from the plasma is the discriminating grid (D), and is where the retarding potential is applied. The fourth grid is called the secondary grid $(\mathrm{S})$ and fulfills two roles: the collected species is accelerated again, and a potential difference higher than the maximum energy of secondary electrons is set with the collector, ensuring that emitted electrons are collected. The contribution of the emitted electrons to the current is thus zero. The structure of the analyzer is presented in figure 2.7 a for positive ion energy analysis, and its implementation is shown in figure 2.7b. The distance between the first grid and the collector is $1 \mathrm{~mm}$. The reject grid $(\mathrm{R})$ is biased at $-50 \mathrm{~V}$, which reflects all electrons since the typical electron temperature is between 3 and $5 \mathrm{~V}$. The discriminating grid (D) is biased between 0 and $100 \mathrm{~V}$. The secondary grid $(\mathrm{S})$ is biased to $-20 \mathrm{~V}$ and the collector to $-10 \mathrm{~V}$ : the resulting $10 \mathrm{~V}$ difference is sufficient to collect all emitted electrons.

As particles are accelerated and decelerated between grids, a collision with another particle would randomly prevent the collection. The mean free path for collisions is the key parameter to determine whether this issue arises or not, and depends on the pressure. A maximum value for the pressure thus corresponds to an analyzer length. Introducing 


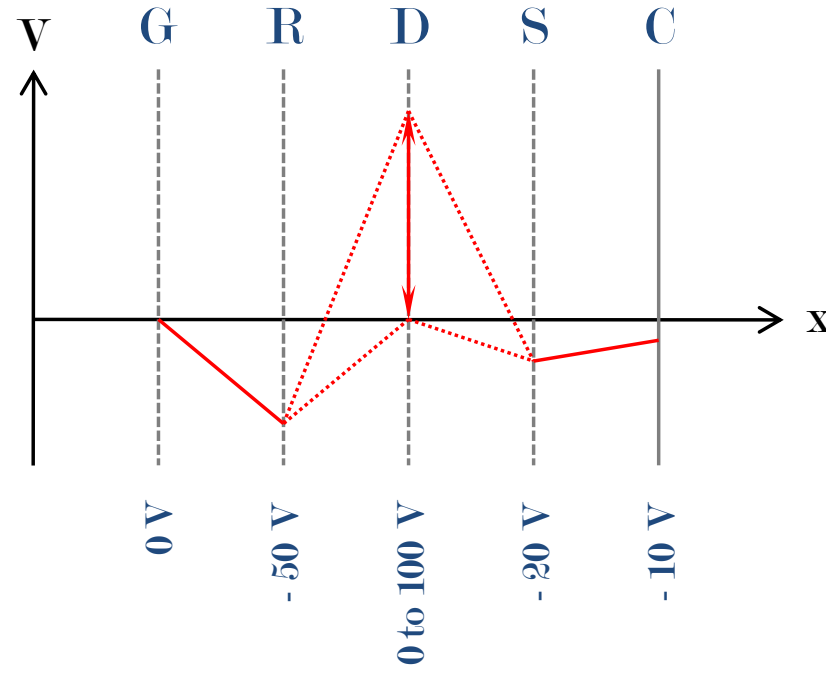

(a)

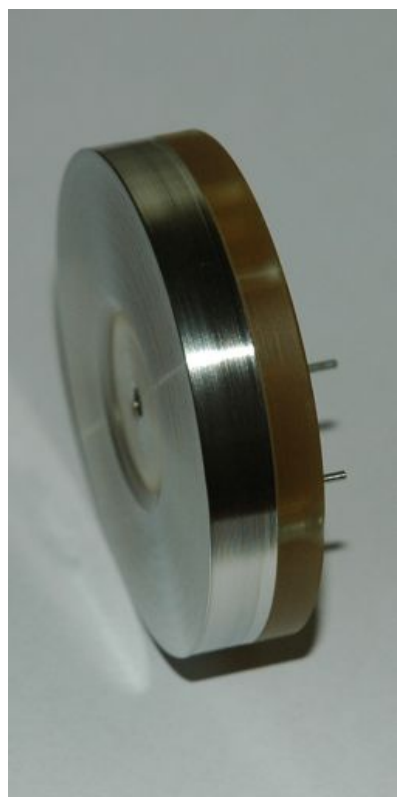

(b)

Figure 2.7: Schematics (a) of a retarding field energy analyzer, and grid and collector biases for positive ion energy measurements: Ground, Reject, Discriminating, Secondary and Collector. Photo (b) of the analyzer with the hole where the particles enter at the center and two of the pins for the polarization of the grids.

a differential pumping in the analyzer is another way of avoiding the problem, and was implemented for the analyzer used in experiments.

\subsubsection{Instruments}

\section{Current Measurement Box}

For Langmuir-type probes and for a retarding field energy analyzer, a current resulting from a bias is to be measured. Good care in designing the circuit should be taken as one can perturb the other. A resistance is introduced in the circuit composed of the probe and the direct-current generator, and placed between the generator and the ground (see figure 2.8a). The current collected with the probe $I_{c}$ is unperturbed and can be obtained from the voltage across the resistance $V_{R}$ using Ohm's law $\left(I_{c}=V_{R} / R\right)$. However, the bias of the probe $V_{\text {bias }}$ is no longer that of the generator $V_{\text {gen }}$, but its combination with the voltage across the resistance

$$
V_{\mathrm{bias}}=V_{\mathrm{gen}}+V_{R}
$$

which can be easily taken into account.

For the implementation of this box (see figure 2.8b), two resistance values can be used: either $100 \Omega$ or $1 \mathrm{k} \Omega$. A stronger signal (tenfold) for the measured voltage across the resistance is obtained with the higher value of the resistance. However, as these resistances are limited power-wise $(250 \mathrm{~mW})$, the resistance with the lower value is able to withstand higher currents

$$
P_{\max }=R I_{\max }^{2}
$$




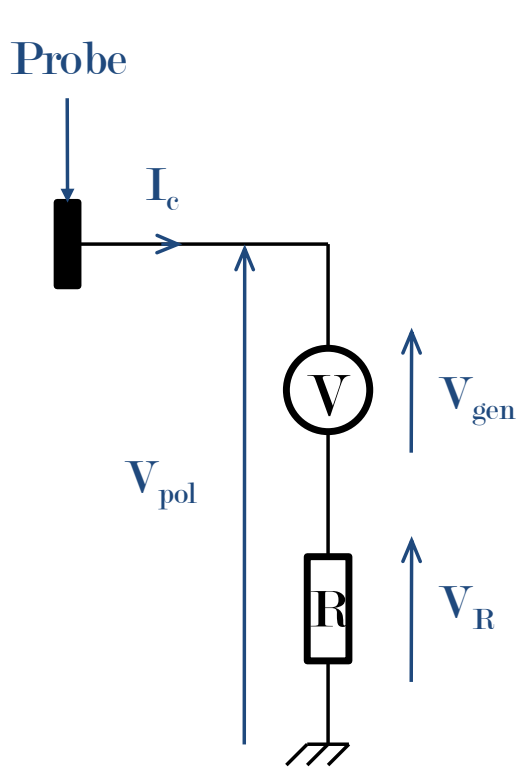

(a)

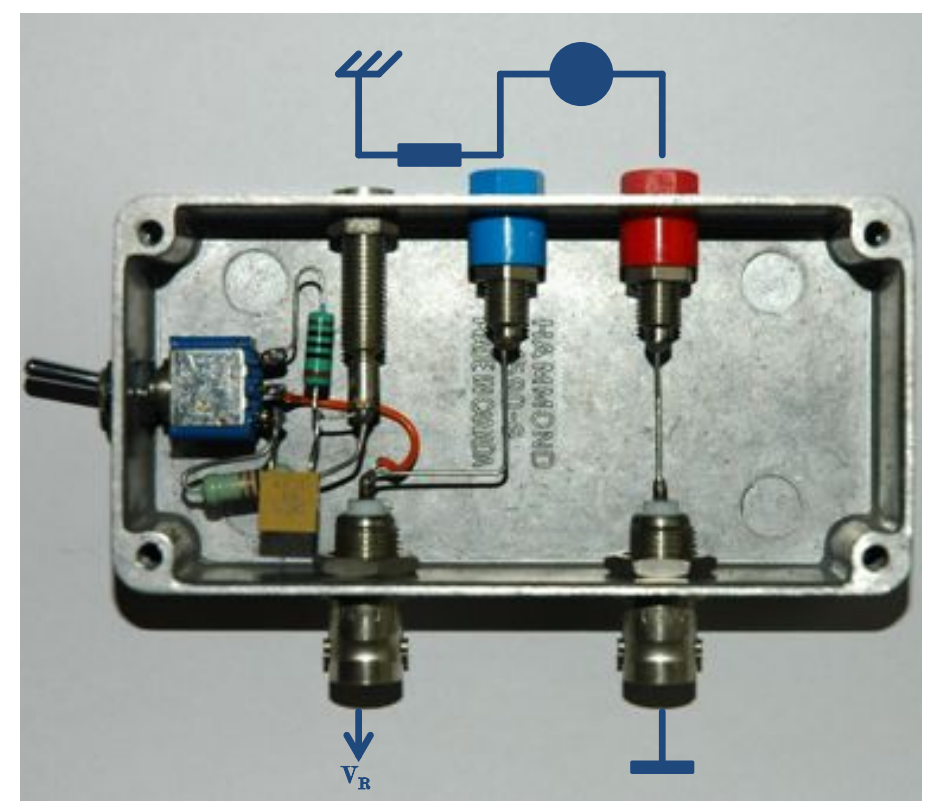

(b)

Figure 2.8: Circuit (a) for the current measurement and implementation (b) of the box.

In some cases, the current measurements need to be averaged. This can be done by placing a capacitor in parallel of the measurement resistance. The characteristic time for this circuit is given by

$$
\tau=R C,
$$

which gives the minimum frequency that can be considered filtered

$$
f=\frac{5}{\tau}=\frac{5}{R C}
$$

For the mass efficiency measurements presented in chapter 4, this frequency was chosen at $f=50 \mathrm{kHz}$ with a resistance $R=1 \mathrm{k} \Omega$. The value of the needed capacitor is thus $C=100 \mathrm{nF}$.

\section{Devices and Communication Protocol}

The bias of probes is done with a JFA BIP 100-1 direct-current generator, capable of $\pm 100 \mathrm{~V}$ and $\pm 1 \mathrm{~A}$. Although these values may seem high, electronic currents as high as $300 \mathrm{~mA}$ can be collected and a good positive ion current saturation profile might need voltages as low as $-60 \mathrm{~V}$.

A Keithley 2000 multimeter is used as voltmeter. Three averaging modes can be set for this high-precision voltmeter, depending on the timescale to be investigated.

The oscilloscope is a Yokogawa DL 1640. It is capable of a sampling rate as high as $200 \mathrm{MSs}^{-1}$. Using the Nyquist-Shannon sampling theory, the maximum frequency for observed signals is $100 \mathrm{MHz}$. Mathematical functions, such as fast Fourier transform (FFT), are available for signal analysis.

For computer programs, described in section 2.2.5, to be able to control and record measurements, a way of communicating between devices is needed. As the GPIB protocol 
(IEEE-488) is available on all devices, it is chosen as communication protocol. A GPIB card installed in the computer allows it to be linked to measurement devices.

\subsubsection{Computer Programs}

For speed and accuracy, electrical measurements involving I-V characteristics or energy analysis are done with computers. A commercial system, Scientific Systems, provides a complete analysis of I-V characteristics with a box containing measurement circuits and a direct-current generator, which is linked to a computer for the interface. Computer programs were developed in the lab for Langmuir-type probes and the retarding field energy analyzer.

\section{Scientific Systems}

It is an automated system to obtain and analyze I-V characteristics, to be later saved on a computer. A single box contains all devices necessary to polarize the probe and measure the resulting collected current. The connection between the probe and the box is a coaxial cable, and the one between the box and the computer is a specific cable for which a proprietary card is needed.

Any characteristic is recorded on 201 points for a specified voltage range in the interval $[-95,95] \mathrm{V}$, which means that the voltage step depends on the voltage range. Even when filtering inductances are used, the characteristics still need to be averaged as frequencies below the excitation frequency are present in the plasma. Two averaging methods can be used and combined:

- \# sample per point: for each polarization value, the collected current value can be taken several times,

- \# sweep per characteristic: the entire I-V characteristic is taken several times.

Both methods are usually combined and the final $\mathrm{I}-\mathrm{V}$ characteristic corresponds to a double averaging.

The analysis of I-V characteristics, which follows the description in section 2.2.1, was designed for filament probes as the calculation of the probe area requires the length and radius of the filament. Additionally, the mass of the main positive ion is needed. In plasmas created in molecular gases, such as sulfur hexafluoride $\left(\mathrm{SF}_{6}\right)$, the main positive ion is not obvious and needs to be determined, with a mass spectrometer for instance. As with any measurement, noise is present with the signal, which is problematic since a second derivative is needed to determine the plasma potential. The $n^{\text {th }}$ derivative of a signal with a frequency $\omega$ is amplified as follows

$$
\frac{d^{n}}{d t^{n}}(A \cos (\omega t+\phi))=A \omega^{n} \cos \left(\omega t+\phi+\frac{n \pi}{2}\right) .
$$

This means that the second derivative of the signal is often lost in the second derivative of the noise. To solve this problem, a Savitzky-Golay smoothing filter is used [36]. At every point of the I-V characteristic, the curve is locally fitted with a polynomial curve of order $n$ (order of the derivative) on at least $2 n+1$ points centered around the current point.

$$
P=\sum_{j=0}^{n} c_{j} x_{i}^{j} .
$$


with $x_{i}$ the points of the I-V characteristic. The value of the $n^{\text {th }}$ derivative on the center point $i=0$ can then be obtained with

$$
x_{0}^{(n)}=n ! c_{n}
$$

The polynomial fit is done with a least-squares fit that minimizes the total mean-square error.

\section{Lab-made Programs}

All programs were written in $\mathrm{C}++$ with a GPIB library obtained with the Yokogawa oscilloscope. The first instances of the Langmuir-type probe and RFEA programs were written by Nicolas Plihon during his $\mathrm{PhD}$ thesis [21], which were then modified. Additional programs were also written to control other devices.

The Langmuir-type program was written to overcome the limitation of the Scientific Systems box which requires the user to start the system for each characteristic. Its analysis part was first compared to the automated analysis and showed the same results for the same Savitzky-Golay parameters (order of the polynom, number of points). It should be noted that these parameters can be freely adjusted with the program, as tuning might be required for a better analysis, contrary to the automated system.

The principle of the retarding field energy analyzer is very similar to Langmuir-type probes: measurement of a collected current for a range of biases. The end result, however, is the first derivative itself.

\subsection{Electronegativity Measurement Techniques}

\subsubsection{Review of Techniques}

In an electronegative plasma, a third species, the negative ions, takes part in the plasma equilibrium. There are now two negatively charged species, the negative ions and the electrons, with a high mass ratio $\left(\sim 3 \times 10^{4}\right.$ for $\left.\mathrm{O}^{-}\right)$. Therefore, the electronegativity $\alpha=n_{-} / n_{e}$ is a key parameter in determining which species dominates in the electrical equilibrium. For instance in an ion-ion plasma (electron free), $\alpha \rightarrow \infty$. Several diagnostics are available to measure the negative ion density:

- laser-induced photodetachment,

- mass spectrometry,

- ion acoustic wave measurement,

- electrostatic probe (used in this thesis).

\section{Laser-Induced Photodetachment}

The laser-induced photodetachment technique [37, 38] consists of measuring the electron density with a Langmuir-type probe before and during a laser pulse. The role of the laser is to detach electrons from negative ions, which means that the laser frequency should be chosen carefully to ensure that all negative ions are detached, regardless of energy. The surplus of electron density during the pulse thus corresponds to the density of negative 
ions. The electron density is measured with a Langmuir-type probe placed in the laser beam. As both electron and negative ion densities are measured, the electronegativity can be obtained directly.

However, this diagnostic is difficult to use in a small device because of the laser which must be calibrated and aligned. Additionally, windows are required in the plasma reactor for the laser beam to reach the region to be investigated, as well as the Langmuir-type probe which should be placed carefully in the laser beam.

\section{Mass Spectrometry}

Mass spectrometers can separate charged particles by mass and charge. Several designs are possible:

- sector type: the charged particle goes through a region with a static magnetic field and its trajectory change, due to the Larmor radius which depends on the charge and mass of the particle, is measured,

- time of flight: the charged particle goes though a region with a constant electric field and its time of flight and velocity, depending on the charge and mass, are measured,

- quadrupole: a radio-frequency quadrupole field is set in a region which the charged particle traverses, with only one mass to charge ratio being able to pass through, meaning that only ions fulfilling this condition are collected.

With these diagnostics, negative ions can be identified precisely, which is especially useful for plasmas in molecular gases where several species of ions are present: the main negative ion can be determined.

However, these diagnostics are usually placed outside of plasma reactors and can seldom be placed in the volume of a discharge, hence there is no spatial resolution. As described in section 2.1.2, the potential near the walls in a plasma reactor is usually created to accelerate positive ions and retard electrons because of quasi-neutrality. This means that extracting negative ions is only possible under certain conditions: either by studying a plasma where the sheath is governed by negative ions and not electrons in the case of high electronegativity [39] or by pulsing the discharge [40]. Moreover, mass spectrometers are heavy to use.

\section{Ion Acoustic Wave}

The calculation of the phase velocity of ion acoustic waves shows that it is dependent on the positive ion to negative ion density ratio for both fast and slow modes [41]. In the case of the fast mode, plasma antennae can be used to determine the phase velocity [42].

However, this technique requires additional measurements with Langmuir-type probes to determine the electron temperature (a parameter of the phase velocity expression) and the positive ion to electron density ratio. Similarly to mass spectrometry, spatial resolution is difficult to achieve because of the measurement method. In this technique, a propagating wave is measured.

\section{Electrostatic Probe}

The technique relies on the analysis of an I-V characteristic measured by a Langmuir-type probe., and was for instance used in Nicolas Plihon's PhD thesis [21]. Langmuir-type 
diagnostics are easy to use and cheap to implement. The principle of the technique is presented in the next section.

Since Langmuir-type probes are used, measurements are difficult in highly reactive plasmas due to the deposition on or the destruction of the probe itself, and in highly magnetized plasmas due to the underestimation of the current of magnetized species.

\subsubsection{Electrostatic Probe}

\section{Principle}

This technique was developed by Pascal Chabert during his PhD thesis [43], and was used to characterize a sulfur hexafluoride helicon discharge [44].

In electronegative plasmas, the positive ion flux at the entry of a sheath is modified by the presence of negative ions at the sheath edge. The Bohm velocity, minimum velocity of positive ions entering the sheath, is then modified. A measurement of positive ion current, therefore, contains information on the electronegativity $\alpha$, and can be expressed as

$$
I_{\text {sat }}^{+}=e \mathcal{A}_{1} \Gamma_{s}(\alpha)
$$

with $\mathcal{A}_{1}$ the area of a (usually planar) probe and $\Gamma_{s}$ the positive ion flux at the sheath entry. As it was described in section 2.2.1, a planar probe is the best choice for an accurate measurement of the ion saturation current, especially in the case of a guarded planar probe. The positive ion density depends on the parameter of the discharge and should be normalized. This is done by considering the electron flux. The current collected at the plasma potential is a combination of all species currents as there is no retarding field, and can be written as

$$
I\left(V_{p}\right)=\frac{1}{4} e \mathcal{A}_{2}\left(-n_{e 0} v_{e, \mathrm{th}}-n_{-0} v_{-, \mathrm{th}}+n_{+0} v_{+, \mathrm{th}}\right) .
$$

The considered velocities are thermal velocities because there is no electric field as species are collected: $v_{\mathrm{th}}=\sqrt{8 e T /(\pi m)}$. The factor $\frac{1}{4}$ comes from the fact that a threedimensional distribution function is integrated to obtain the flux in one direction. Since there is no sheath, the area of collection is the area $\mathcal{A}_{2}$ of a (usually filament) probe. With an electron current collected, a filament probe is preferred. The contribution of the ion currents is negligible for $\alpha<100$ because of the difference in temperatures and masses. Using $c_{s}=\sqrt{e T_{e} / m_{+}}$the ion acoustic speed in the absence of negative ions, the ratio of currents $R_{\text {gas }}$ for a specified feedstock gas thus is

$$
R_{\mathrm{gas}}=\frac{I\left(V_{p}\right)}{I_{\mathrm{sat}}^{+}}=\left(\frac{\mathcal{A}_{2}}{\mathcal{A}_{1}}\right) \sqrt{\frac{m_{+}}{2 \pi m_{e}}} \frac{n_{e 0} c_{s}}{\Gamma_{s}(\alpha)} .
$$

A model of an electronegative discharge is now needed to express the ratio of electron flux $n_{e 0} c_{s}$ to the flux of positive ion flux $\Gamma_{s}(\alpha)$. This model was developed by Sheridan et al and its complete description is presented in [39]. A one-dimensional electronegative plasma is considered with electrons, positive ions and negative ions. Both electrons and negative ions are assumed to be in Boltzmann equilibrium but positive ions are considered cold (inertia effect and no pressure). With this model, the ratio of positive ion to electron flux can be solved analytically for two cases: small electronegativity $(\alpha \ll 1)$ and high 


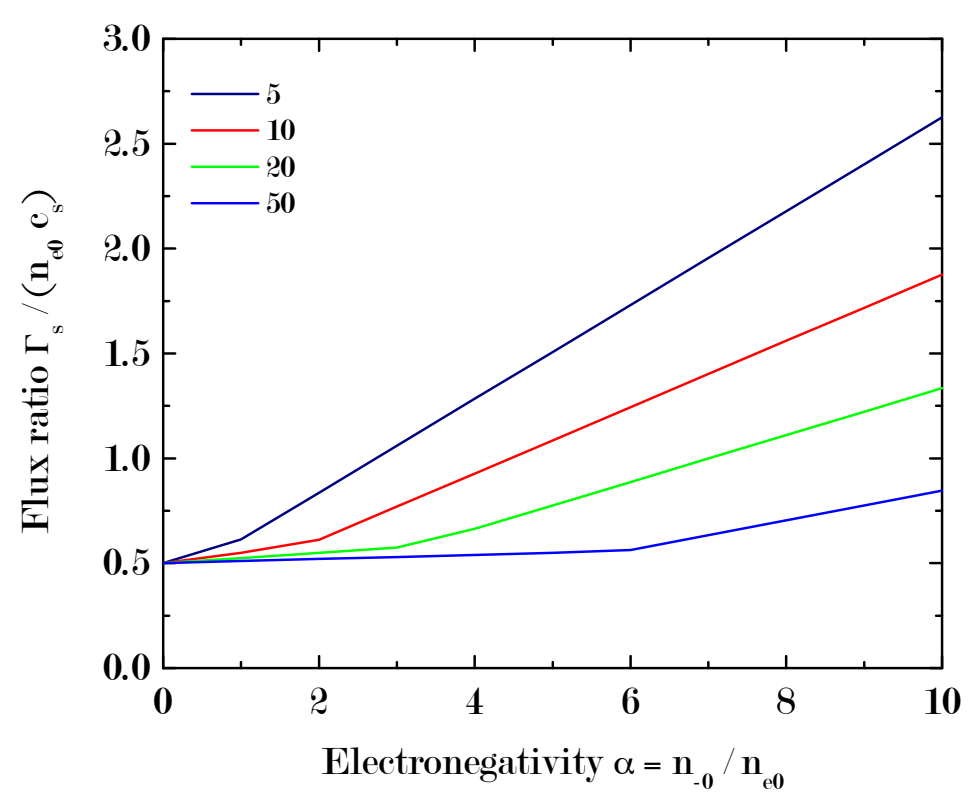

Figure 2.9: Ratio of positive ion flux at the sheath to electron flux versus the electronegativity $\alpha=n_{-0} / n_{e 0}$ for different $\gamma=T_{e} / T_{-}$from 5 to 50 .

electronegativity $(\alpha \gg 1)$. This ratio is a function of electronegativity $\alpha$ and ratio of electron to negative ion temperature $\gamma$

$$
\frac{\Gamma_{x}}{n_{e 0} c_{s}}= \begin{cases}\frac{1}{2}+\frac{1}{2 \gamma} \alpha & \alpha \ll 1, \\ \frac{1}{\sqrt{\gamma}}\left[\left(\frac{1}{2}\right)^{1 / \gamma}+\frac{1}{2} \alpha\right] & \alpha \gg 1 .\end{cases}
$$

The ratio of fluxes is shown in figure 2.9 as a funtion of electronegativity for ratios of temperatures $\gamma \in[5,10,20,50]$. The first part of each curve before the knee corresponds to the $\alpha \ll 1$ case, and the second part after the knee to $\alpha \gg 1$. Because the plasma potential at the sheath edge is double-valued in the first part of the curves, the model is only valid in the second part. It should be noted that in the case of an electropositive plasma $(\alpha=0)$, this ratio is $\frac{1}{2}$.

\section{Limitations}

The first limitation comes from the ratio of probe areas in equation (2.37). In theory, between the fact that there is no sheath for $V=V_{p}$ and the fact that a guarded planar probe cancels sheath effects on the current collection, this ratio is correct. However, the uncertainty around the exact plasma potential value (determination from a second derivative on a noisy signal) and the fact that a guarded planar probe is not perfect mean that there are sheath effects and that the area of current collection for both probes is not the probe area. A way of determining the ratio of collection areas $\left(\frac{\mathcal{A}_{2}}{\mathcal{A}_{1}}\right)^{*}$ is thus needed. Equation (2.38) shows that for an electropositive plasma $(\alpha=0)$, the ratio of fluxes $\left(n_{e 0} c_{s}\right) / \Gamma_{s}$ is constant at $\frac{1}{2}$. Assuming that the sheath size is the same for the measured gas and argon, the ratio of collection areas can be determined by measuring the ratio of 
currents in argon

$$
R_{\text {argon }}=\sqrt{\frac{2 m_{+}}{\pi m_{e}}}\left(\frac{\mathcal{A}_{2}}{\mathcal{A}_{1}}\right)^{*}=216\left(\frac{\mathcal{A}_{2}}{\mathcal{A}_{1}}\right)^{*} .
$$

The validity of the model $(\alpha \gg 1)$ comes from the structure of an electronegative plasma which depends on the electronegativity. For low electronegativity, the equilibrium is governed by the electrons, and negative ions are confined to the center of the discharge. The plasma near the walls is an electropositive plasma. For an intermediate electronegativity, negative ions dominate in the center of the discharge, but as the ion densities decay toward the walls, the electrons take over and oscillations can be seen near the edge until the Bohm velocity is reached for positive ions. For high electronegativity, negative ions dominate the entire discharge. The positive ion flux at the sheath entry depends strongly on the negative ion density. The model is only valid in the last case. Depending on the value of $\gamma=T_{e} / T_{-}$, there is a minimum value of $\alpha$ for the model to be valid. For example with $\gamma=50$, it can be seen in figure 2.9 that this minimum value is $\alpha=6$. Any electronegativity below this value cannot be investigated.

Finally, the relation between the positive ion to electron ratio $\Gamma_{s}(\alpha) /\left(n_{e 0} c_{s}\right)$ shows a strong dependence on $\gamma$. As the negative ion temperature is difficult to determine in most cases, it is a limiting factor for the electrostatic probe measurement of electronegativity. 


\section{Part I}

\section{Experiments}





\section{Chapter 3}

\section{Experimental Setups}

\section{Contents}

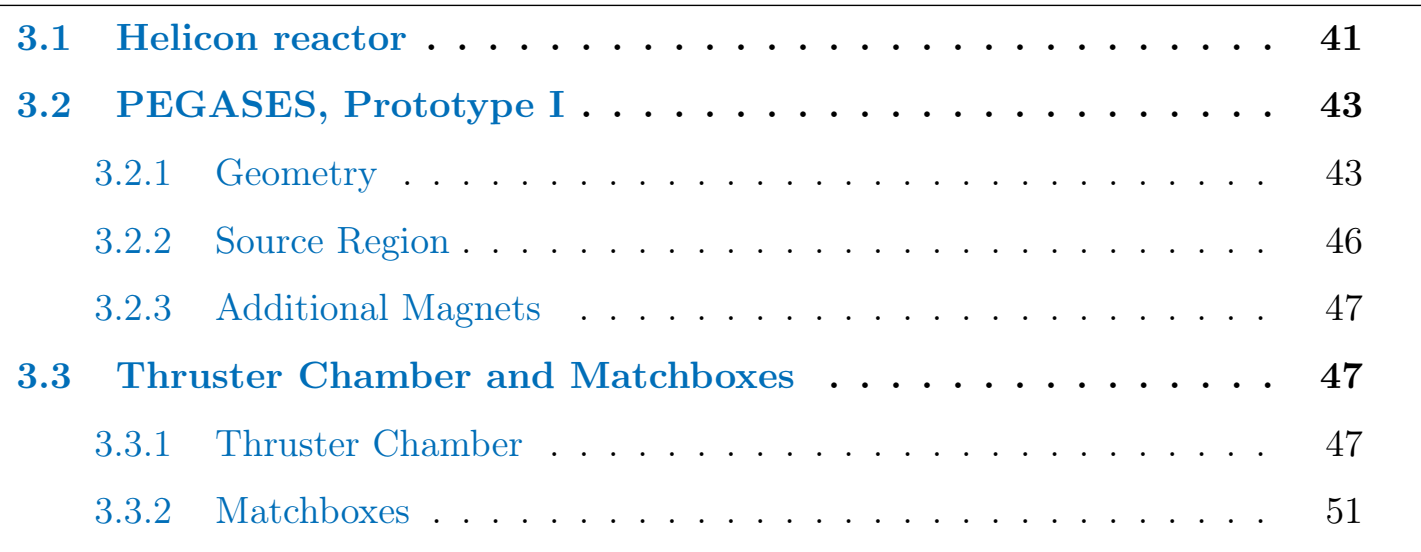

The idea behind PEGASES came from the study of the structure of an electronegative discharge in the helicon reactor in LPP [44, 21, 45]. It was indeed observed that an ion-ion plasma is created in the periphery of the diffusion chamber when electrons are confined to magnetic field lines but not ions. Further studies were done in the helicon reactor during this thesis to characterize an ion-ion plasma and its formation. These would help in the design of the first PEGASES prototype.

The PEGASES thruster is housed in a vacuum chamber, on which probes and analyzers can be connected. The power is coupled to the antenna via a matchbox.

All these setups are described in this chapter, before the presentation of the experimental results in chapters 4 and 5 .

\subsection{Helicon reactor}

This reactor, shown in figure 3.1, has a cylindrical geometry and is divided into two chambers: a source chamber on top of a diffusion chamber (see figure 3.1a). The turbo pump is connected sideways above the source chamber and the gas feed at half height of the diffusion chamber. Without power applied, the neutral gas is introduced in the diffusion chamber, goes up through the source chamber and is extracted above. The volume of the helicon reactor, shown in figure 3.1b, is $24 \mathrm{~L}$.

Two sets of two coils can be used to create a static magnetic field, one around each chamber. In the diffusion chamber, the magnetic field on the axis in gauss was calculated 


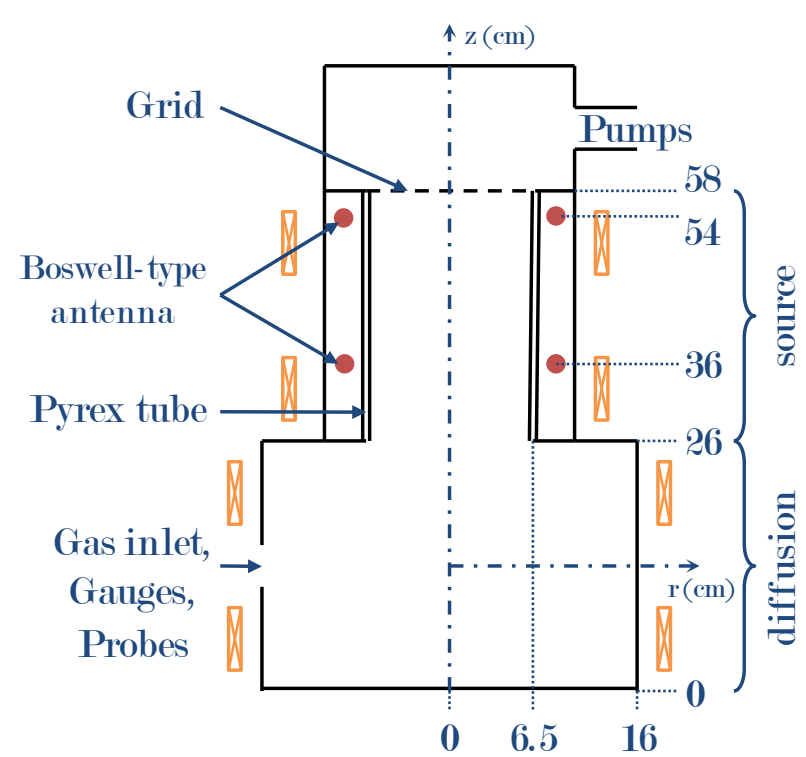

(a)

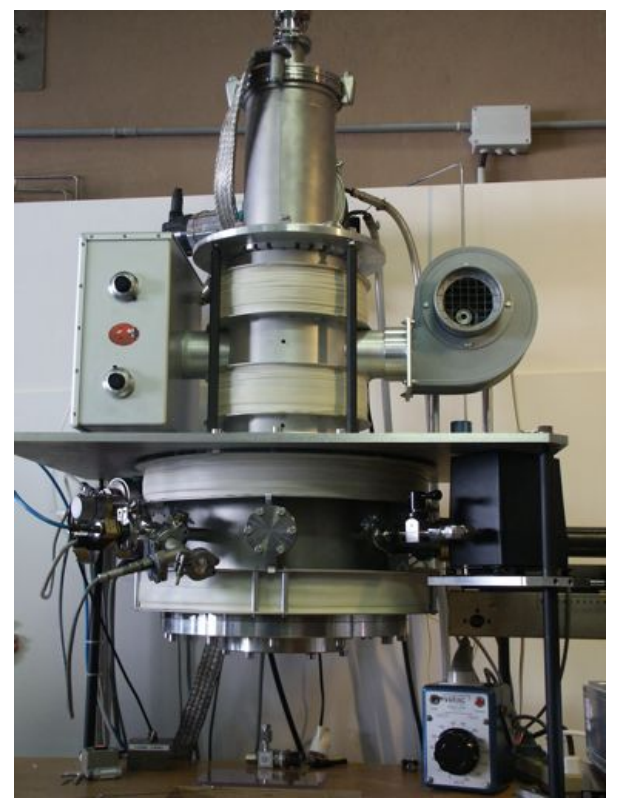

(b)

Figure 3.1: Schematics (a) and photo (b) of the helicon reactor.

as a function of coil current in ampere

$$
B(G)=14 \times I(A) .
$$

With a high enough current, the coils can heat up quickly to a temperature much higher than the room temperature. In order to limit the influence of such temperature on the plasma through the walls, the coils are set at a distance from the walls $(3 \mathrm{~cm})$.

The plasma is created with a Boswell-type antenna, wrapped around the pyrex tube of the source chamber, and excited at $13.56 \mathrm{MHz}$. Without a static magnetic field, the power coupling can be either capacitive or inductive. When a static magnetic field is applied, helicon waves can be launched in the source chamber. The antenna is a copper sheet of dimensions $10 \times 1.2 \mathrm{~mm}$ and follows the design described by Boswell [11], which is capable of exciting different modes of helicon waves (see section 4.1.1). It is cooled by a fan located to the side, which also cools the pyrex tube of the source chamber. The radio-frequency generator is an RFPP RF30H, with a maximum power output of $3 \mathrm{~kW}$ and pulsing capabilities. The output impedance of the generator is matched to that of the plasma with a matchbox using an L-circuit (see section 3.3.2).

Pressure measurements are made with an MKS baratron connected at half height of the diffusion chamber. This gauge, with a maximum measuring pressure of 1 Torr, consists of a capacitance measurement between a membrane and a reference surface. The vacuum is obtained with two pumps, a primary pump and a turbomolecular pump. The primary pump is a Leybold rotary vane vacuum pump (with Fomblin as a pumping fluid) capable of pumping $40 \mathrm{~m}^{3} \mathrm{~h}^{-1}$. The turbomolecular pump is an Alcatel ATP 400C with a pumping capability of $400 \mathrm{Ls}^{-1}$. This combination results in a background vacuum of $2 \times 10^{-6}$ mbar, with an intermediate pressure around $10^{-3}$ mbar between the two pumps. A floating grid is placed above the source chamber to neutralize the plasma before it reaches the turbomolecular pump. The gases used for experiments were argon $(\mathrm{Ar})$, oxygen $\left(\mathrm{O}_{2}\right)$ and sulfur hexafluoride $\left(\mathrm{SF}_{6}\right)$. As oxygen and sulfur hexafluoride are corrosive gases, a 


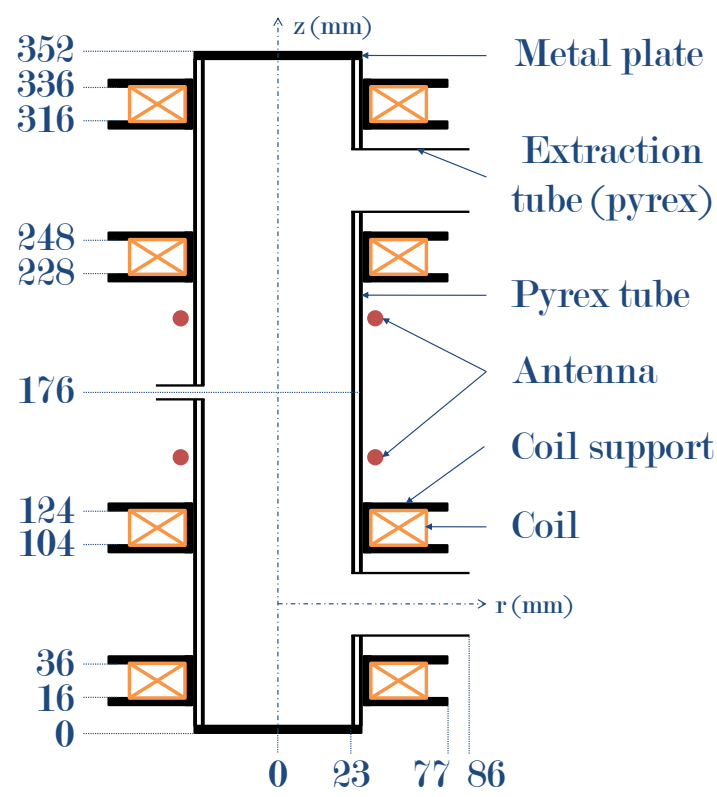

(a)

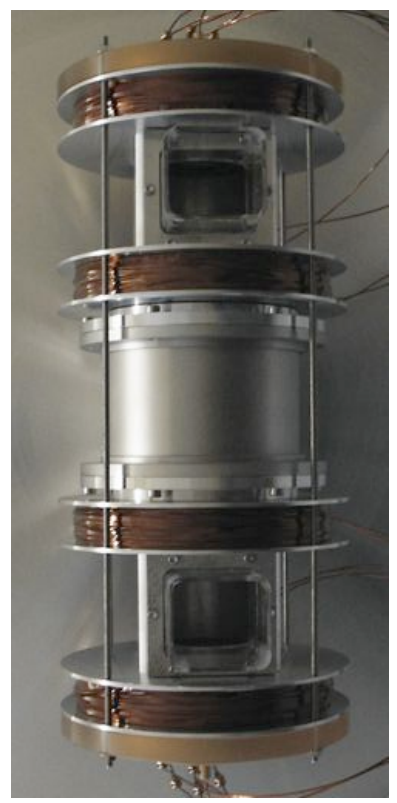

(b)

Figure 3.2: Prototype I of the PEGASES thruster: schematics (a) and photo (b).

turbomolecular pump is needed. Nitrogen $\left(\mathrm{N}_{2}\right)$ is introduced in the pump as sealing gas to protect it, especially in the case of oxygen.

Probes can be mounted at half height of the diffusion chamber through two vacuum connections facing each other. Two glass/pyrex windows, also at half height and facing each other, can be used for optical diagnostics.

\subsection{PEGASES, Prototype I}

\subsubsection{Geometry}

To design the PEGASES thruster prototype, the ion-ion plasma creation in the helicon reactor needs to be understood. The plasma is created in the source region and expands in the diffusion region, whose diameter is much bigger than the source region $(32 \mathrm{~cm}$ compared to $13 \mathrm{~cm}$ ). As the feedstock gas is electronegative, electrons, positive and negative ions are created. However, the electronegativity $\alpha$ is usually low and the electrons dominate the electrical equilibrium. Without a downstream static magnetic field, this plasma would diffuse in the bottom chamber with little change in its composition. The introduction of a static magnetic field, however, changes that. Its strength is chosen so that electrons are magnetized but ions are left unmagnetized. The electrons are created in a column roughly the same diameter as the source region, in either the source or the diffusion region. The fact that electrons are not only created in the vicinity of the antenna is due to the wave heated power coupling: the helicon wave propagates in the column. In the diffusion region, the periphery of the column is not easily accessible to electrons, while ions can diffuse without magnetic confinement as long as quasi-neutrality is fulfilled.

The design of the first PEGASES prototype, shown in figure 3.2 (schematics (a) and photo (b)), is largely based on the helicon reactor. In order to leave the matter of the ion acceleration open, two extraction zones are set. For instance, positive ions could be 
extracted in one and negative ions in the other. For the sake of symmetry, the choice of a double helicon is made: the source region is flanked by two diffusion regions, with one on either side. As with the helicon reactor, the geometry is cylindrical. However, the diameter is kept constant between the source and diffusion regions. Two openings, one in each diffusion region, are made and allow the plasma to diffuse radially into the extraction tubes. The cylinder is made of pyrex (insulating walls), as in the source chamber of the helicon reactor, with an outer diameter of $6 \mathrm{~cm}$ and an inner diameter of $4.6 \mathrm{~cm}$. The inner cross-section of the extraction tubes is a $4.4 \times 4 \mathrm{~cm}$ rectangle with a $2 \mathrm{~mm}$ wall thickness. The total volume of the thruster (cylinder + extraction tubes) is $0.84 \mathrm{~L}$.

As described previously, the static magnetic field is an essential part in the PEGASES design. Not only does it act as an electron filter which confines electrons and allows ions, both positive and negative, to diffuse into an ion-ion plasma region, but it is also a necessary condition for the helicon wave to exist in the plasma, resulting in a high density plasma. The ideal situation would be to create a static magnetic field where all field lines are parallel to one another: the column where electrons are created and confined would be easier to control. Such a situation is possible with a solenoid around the cylinder containing the plasma and on the condition that that solenoid is longer than the cylinder in order to avoid edge effects near the solenoid ends. However, the solution of the solenoid cannot be used as ions are to be extracted radially. A set of four coils is thus placed symmetrically around the cylinder (see figure 3.2): two extraction zones are readily available, and the connection to the antenna is simpler since it can be direct. A set of two coils would be easier to implement, but the divergence of the static magnetic field would be stronger. Permanent magnets were excluded, as the direction and strength of the magnetic field cannot be changed, contrary to coils, where the direction and amplitude of the current can be used as parameters to set the magnetic field. Moreover, the current can be set independently in each coil, allowing the possibility of a variety of magnetic configurations, as can be seen in figure 3.3. These magnetic field simulations, performed with the FEMM software [46], show the magnetic field lines and the magnetic flux density for three coil configurations of the PEGASES thruster. In figure 3.3a, the current is in the same direction for all the coils. Thus, the magnetic field lines in the thruster cylinder as roughly parallel to one another. In figure 3.3b, the current in the two coils to one side of the antenna is opposite to the current in the two coils on the other side of the antenna. This creates a zero of magnetic field at the center of the thruster cylinder. In figure 3.3c, each coil has a current opposite to its direct neighbors. Therefore, there are three zeros of magnetic field along the axis of the thruster: at the center and at each extraction tube. The magnetic electron filtering can only occur in figures $3.3 \mathrm{a}-\mathrm{b}$ where tha magnetic field lines are perpendicular to the direction of extraction, which means that the case of figure 3.3c cannot be used. The difference between figures 3.3a and 3.3b lies in the source region with parallel field lines in the former, and a zero of magnetic field in the latter.

In order to be close to the ideal situation of parallel magnetic field lines, the choice is made to set the current in the same direction for all coils. Figure 3.4 shows the profile of the magnetic field strength on the cylinder axis for three values of the coil current $(I \in\{1,2,3\} \mathrm{A})$ in this case. The coils are set in a series circuit with a total resistance, including two additional wires to connect the coils to a generator, $R=16.7 \Omega$ at room temperature. This value can go up to $25 \Omega$ rather quickly during an experiment at $I=3 \mathrm{~A}$ as the coil heats up (ohmic heating). As the impedance of the coils changes during an experiment, the setting of the direct-current generator should be chosen with care, especially the maximum voltage. The surface temperature of the coils is monitored 


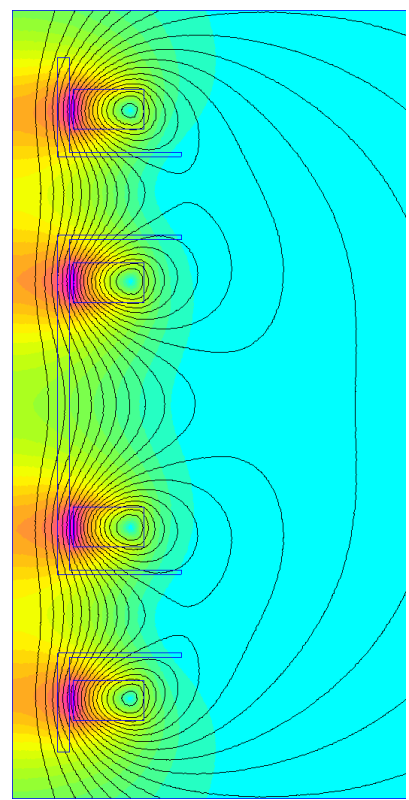

(a)

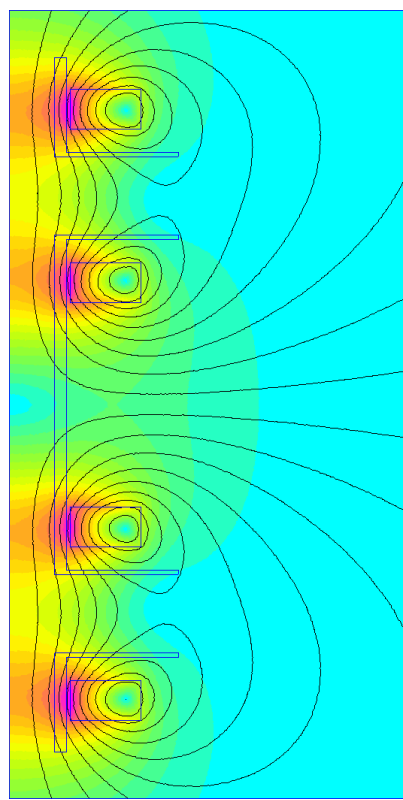

(b)

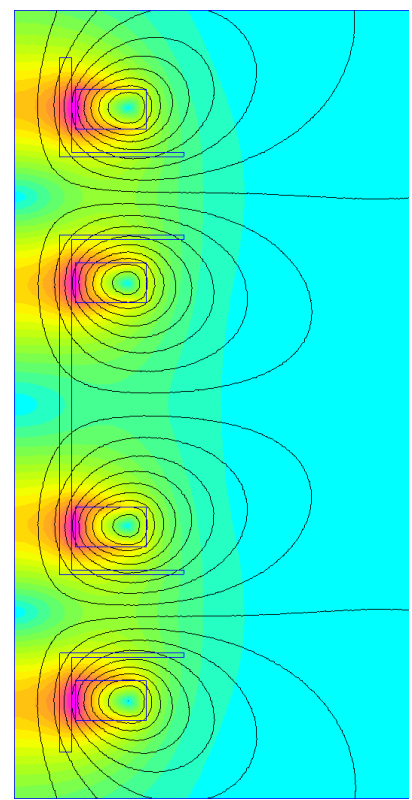

(c)

Figure 3.3: Magnetic field lines and flux density in the PEGASES thruster with the left edge as thruster axis for three coil configurations: (a) the current flows in the same direction for all coils, (b) the current in the two top coils is opposite to that of the two bottom coils, (c) each coil has a current opposite to its direct neighbors. The structure of the thruster (cylinder and the extraction tubes) and the coils is shown in blue lines.

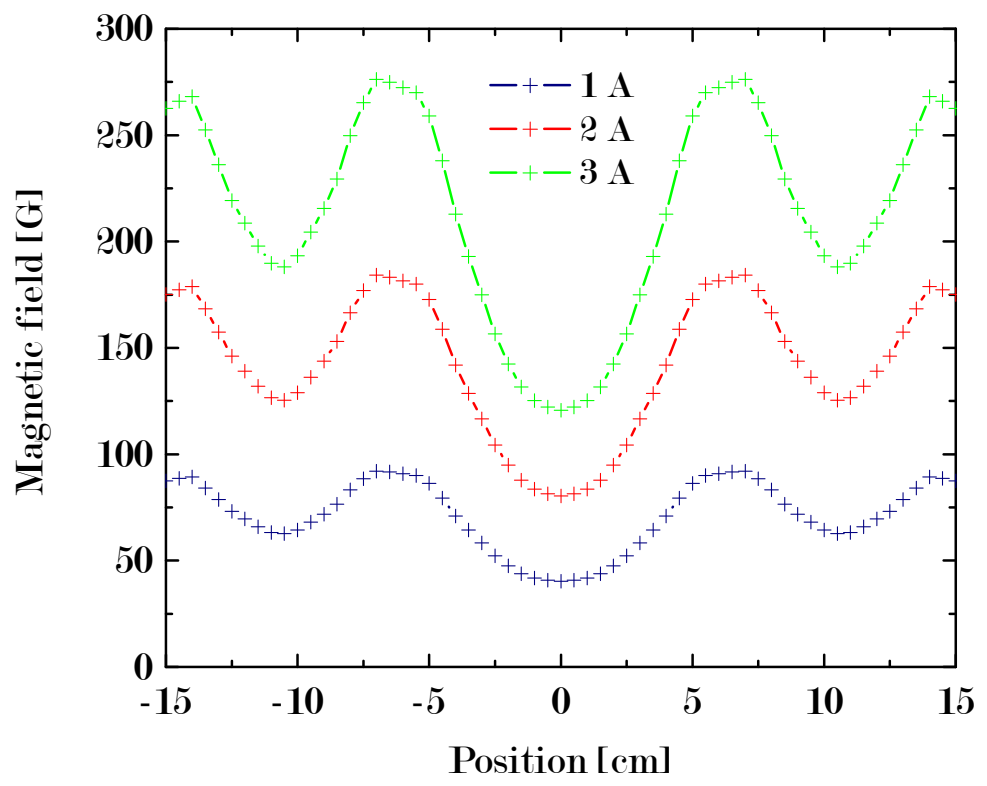

Figure 3.4: Magnetic field on the cylinder axis in Gauss as a function of position for three values of the coil current: $1 A$ (blue), $2 A$ (red) and $3 A$ (green). The maximum value for $I=3 \mathrm{~A}$ is $278 \mathrm{G}$. 
with a thermocouple to prevent overheating. Since the coils are in vacuum, an additional issue arises: any material placed in vacuum is subject to outgassing, which consists of molecules of the material detaching from it. On top of polluting the plasma, the integrity of the coils could be compromised in the case of a high outgassing rate. Kapton insulated copper wires are used because of the low outgassing rate of this polyimide film.

In the case of parallel magnetic field lines, electrons diffuse slowly across them, but travel fast along them. Except near the end walls, the plasma potential, therefore, is constant over the length of each magnetic field line. Metal plates are placed at the ends of the cylinder to independently control the plasma potential. This should be possible as the cylinder is made of insulating material, which means that the potential at the cylinder is not constrained. The magnetic field lines, however, are not parallel because of two factors. First, the inner diameter of the coil corresponds to the outer diameter of the cylinder resulting in edge effects between coils. Second, the strength of the magnetic field is not constant over the length of the cylinder (see figure 3.4), which adds to the divergence of the magnetic field lines.

For a given coil current, the magnetic field strength shows a ratio of 2 between extrema (minimum at the center of the thruster cylinder and maximum at center of the two central coils). Therefore, the magnetic field conditions for experiments are stated with the coil current in amperes. The actual value of the magnetic field can be deduced from figure 3.4.

\subsubsection{Source Region}

As mentioned previously, the source region in the first PEGASES prototype is located at the middle of the cylinder containing the plasma. The antenna, through which the power is coupled, is wrapped around the cylinder. It consists of a copper sheet with its dimensions chosen to support currents at high power. Copper was chosen because of its high conductivity. With a radio-frequency excitation, especially at high power, shielding of the circuit is needed. This is provided by a metal casing around the antenna, connected to a metal panel (wall of the thruster chamber) and a shielded matchbox. The connection between the matchbox circuit and the antenna is done with two copper rods. Since the casing of the thruster is made of metal, short circuits could happen between the antenna and the casing in vacuum. To avoid this issue, the volume between the cylinder and the casing is kept at atmospheric pressure through a connection on the thruster chamber. Early experiments showed that a lot of heat is produced when the power is applied to the antenna. A flow of pressurized air set at 4 bar was introduced next to the antenna as a cooling system. The air flow exits through the matchbox, which is connected on the other side of the thruster chamber panel where the thruster is set.

Two designs of antenna were used: a Boswell-type antenna and a simple three-turn loop antenna. The first design is a downscale of the helicon Boswell-type antenna, with sheet dimensions $8 \times 1 \mathrm{~mm}$ and a average radius of $7 \mathrm{~cm}$ as it is not in contact with the cylinder (polysulfone supports are used to maintain the antenna). It is $6 \mathrm{~cm}$ long, which leaves $1 \mathrm{~cm}$ on each side before the metal casing to avoid short-circuits. The second design consists of a copper sheet of dimensions $7 \times 0.8 \mathrm{~mm}$ wrapped three times around the pyrex tube. The radius is the same as the outer radius of the cylinder: $6 \mathrm{~cm}$. It is shorter than the Boswell-type antenna, being roughly $5 \mathrm{~cm}$ long.

The gas is introduced through a small pyrex tube fused at the center of the main cylinder and connected to a gas feed placed between the copper rods for the antenna. It should be noted that the connection between the two pyrex tubes is a structural weakness, 


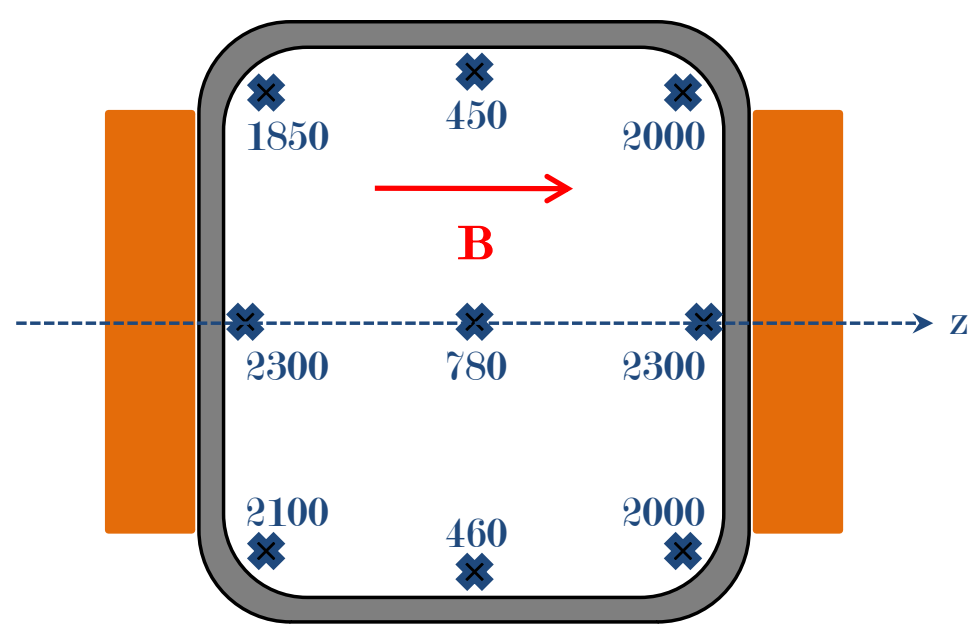

Figure 3.5: Values of the magnetic field in Gauss in the extraction tube with the two permanent magnets shown as rectangles. The $z$-axis represents the cylinder axis, with the center of the thruster on the left hand side, and the side of the thruster on the right hand side.

which requires careful handling when hooking or unhooking the gas connection.

\subsubsection{Additional Magnets}

Along the evolution of the first PEGASES prototype, the decision was made to enhance the magnetic electron filtering by adding permanent magnets in the extraction region. A set of two magnets was placed around one extraction tube, one on each side, as is shown in figure 3.5. The measurements of the magnetic field strength were done with a magnetometer for static magnetic field with a precision of $200 \mathrm{G}$, i.e. the right-center measurement is $2300 \mathrm{G} \pm 200 \mathrm{G}$. This low precision is due to the magnetometer principle: the flux of magnetic field is measured over an area too big to be negligible compared to the dimensions of the extraction tube, and the flux is maximum when the magnetic field is perpendicular to the measurement area, which is difficult to achieve as the magnetic field lines are not parallel, especially near the edges of the magnets.

The permanent magnets create a secondary magnetic electron filter. Electrons are first filtered by the magnetic field created by the coils around the thruster cylinder, and filtered again in the right hand side extraction tube by the magnetic field created by the magnets. The plasma diffusion in the two extraction tubes can be compared to understand the role of the magnetic field in electron filtering (see chapter 5).

\subsection{Thruster Chamber and Matchboxes}

\subsubsection{Thruster Chamber}

In order for the thruster prototype to be operated, a vacuum chamber is needed. This vacuum chamber must house the prototype and probes to be used for measurements, and be connected to a turbomolecular pump, itself connected to a primary pump. A metal grid is placed between the chamber and the turbomolecular pump to neutralize any residual plasma near the pump. The chamber, shown in figure 3.6, consists of a cylinder with 


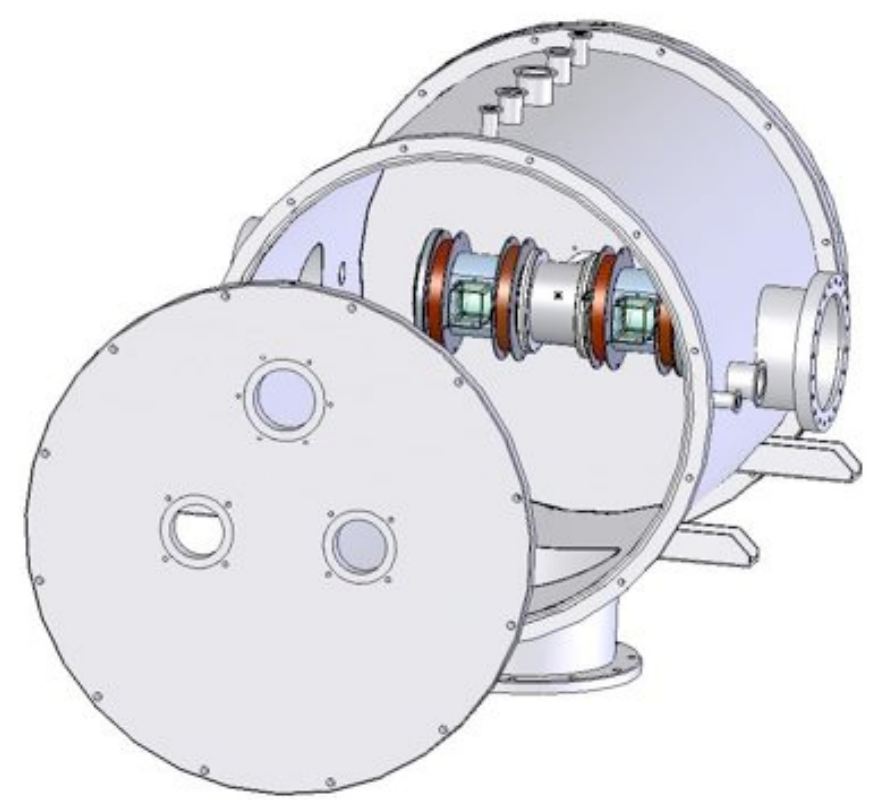

(a)

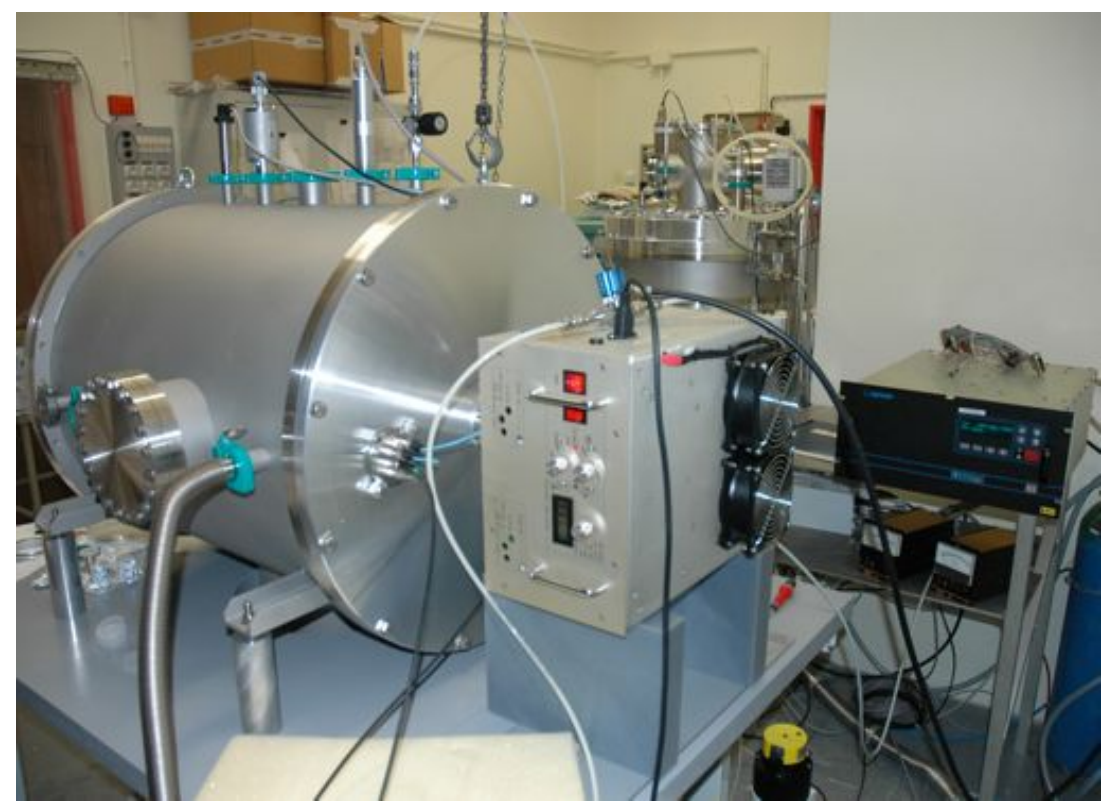

(b)

Figure 3.6: Chamber housing the thruster (a) and view of the chamber and its surroundings (b) with the matchbox (center), the power generator (right hand side) and pressure gauges (on top of the chamber). 
the thruster prototype attached on one end, probes inserted at the other end, and the turbomolecular pump connected underneath at half length of the cylinder. The pressure measurements are set on the opposite side of the pump. Two windows in pyrex are set on each side of the cylinder. The total volume of the chamber, including the tube for the turbomolecular pump and the two windows, is $216 \mathrm{~L}$. The chamber volume is very large compared to the thruster volume for two reasons. First, the end panel opposite to the thruster should be placed as far as possible in order to avoid wall effects, which consist of particles recombining at the wall and being reflected back in the thruster. As a thruster is meant to be operated in space, wall effects need to be negligible. The second reason is that this thruster chamber will be used for further evolutions of the thruster prototype and other prototypes of the PEGASES thruster. As the size and volume of future designs cannot be predicted, these designs are more likely to fit in a larger thruster chamber.

The thruster is fixed at the center of the end panel, with the gas connection and the two copper rods for the antenna passing through an opening. On the other side, the matchbox is connected with a cylinder. This cylinder has two outside connections: one for the feedstock gas and one for the cooling pressurized air. All pieces involved are made out of metal to provide shielding for the radio-frequency power transmitted from the matchbox to the antenna in the thruster. On top of that, a coaxial cable is used to connect the matchbox to the power generator, RFPP RF30H with a maximum output power of $3 \mathrm{~kW}$ and pulsing capabilities.

The vacuum is obtained with two pumps in series: a turbomolecular pump is connected underneath the chamber and a primary (dry) pump to the turbomolecular pump. The turbomolecular pump is a Boc Edwards with a pumping capability of $2500 \mathrm{Ls}^{-1}$. The primary pump is a Boc Edwards dry pump capable of $100 \mathrm{~m}^{3} \mathrm{~h}^{-1}$. These values of pumping speeds are higher than the helicon reactor because the chamber is roughly ten times bigger. Pressure measurements are made with a Pfeiffer Penning gauge for the base vacuum, with a value at $10^{-7}$ mbar, and with a Pfeiffer Pirani gauge between the two pumps, with a value of the order of $10^{-3}$ mbar. A Penning gauge consists of a cold cathode set to a high voltage with a measurement of the ion current, which depends on the pressure. A Pirani gauge is a single wire heated by a current: its temperature is related to the pressure of the gas surrounding it, as the thermal conductivity of a gas depends on the pressure. The operating pressure is measured with a MKS baratron gauge (see 3.1) with a maximum value of 0.1 Torr.

As the gas is introduced into the thruster cylinder, it first expands in the thruster volume $(0.84 \mathrm{~L})$ and then in the chamber volume $(216 \mathrm{~L})$. The resulting pressure in the thruster and in the chamber, therefore, are different, with a higher pressure in the thruster. The pressure measurements with the baratron gauge are thus underestimations of the actual pressure in the thruster. The value of the entering gas flow in standard cubic centimeter $(\mathrm{sccm})$ is chosen as parameter instead of the measured pressure. The pressure in the chamber as a function of the gas flow rate is shown in figure 3.7. It can be seen that the relation is fairly linear. The gas input is controlled by a shielded massflow with a maximum output of $100 \mathrm{sccm}$ for argon, and a scaled maximum of $144 \mathrm{sccm}$ for oxygen.

The end panel opposite to the one with the thruster is where all electrical probes are connected. Figure 3.8 shows two Langmuir-type probes (filament to the left and planar to the right) installed on this end panel. Both probes can be moved along their axis, allowing spatial scans. As it can be seen in figure 3.6, the holes are set in front of the two extraction tubes: probes can thus be placed along the radius of the thruster either inside or outside. The third hole is a pyrex window through which the thruster can be observed 


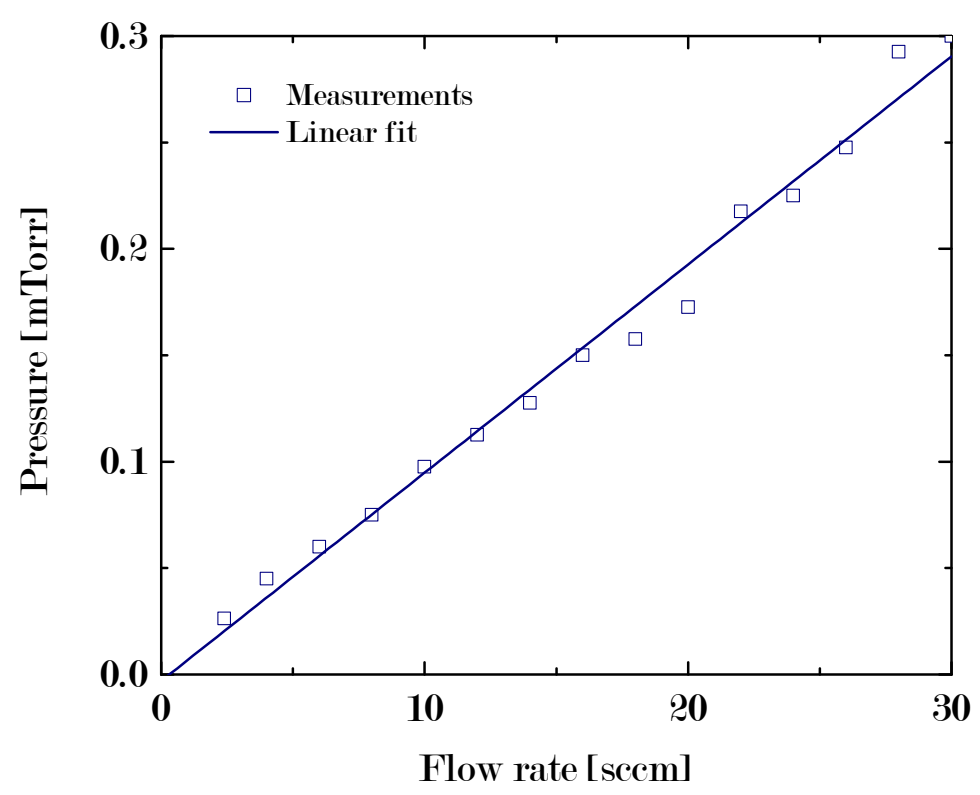

Figure 3.7: Pressure in the thruster chamber in mTorr versus the gas flow rate in sccm. The squares correspond to measurement points, and the line to a linear fit.

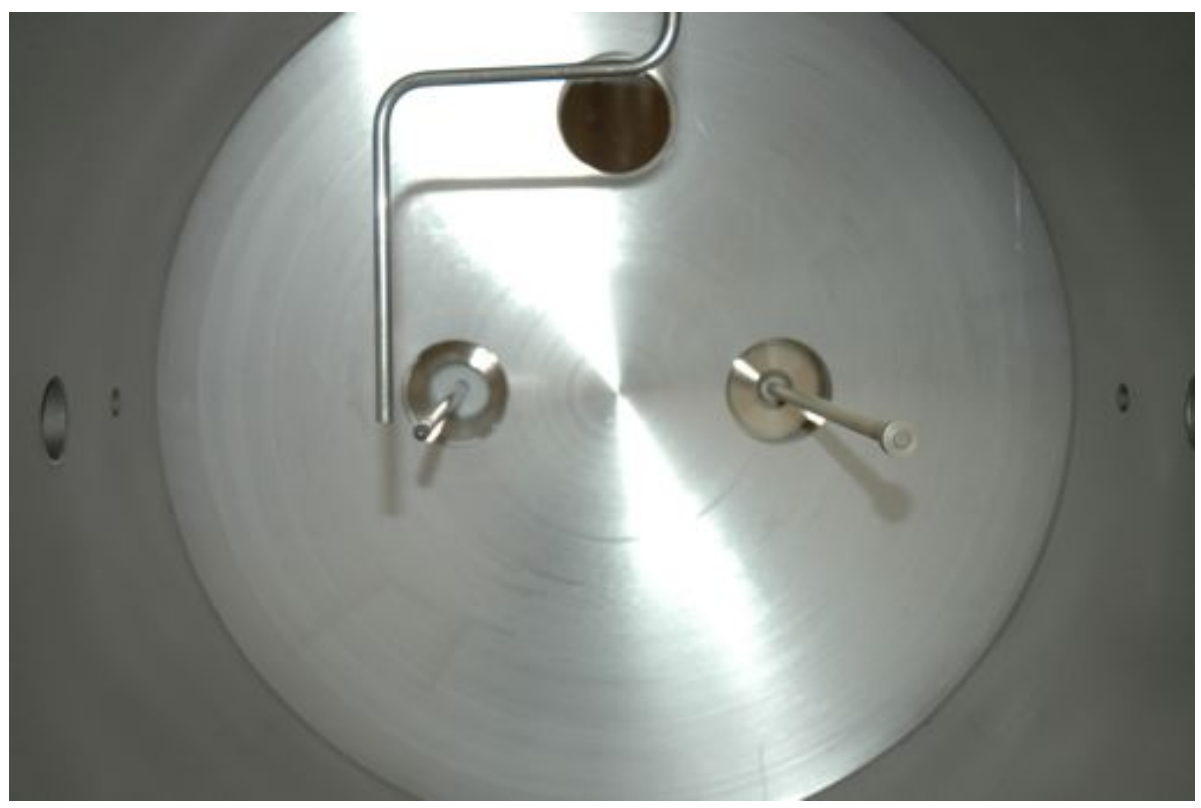

Figure 3.8: Filament probe (left) and planar probe (right) installed on the end panel of the thruster chamber. 


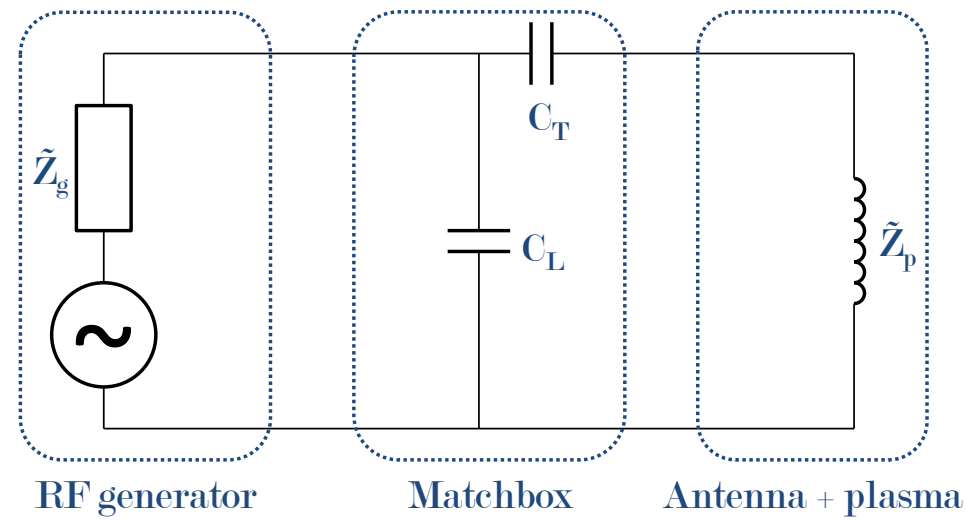

Figure 3.9: Circuit for the power coupling with the radio-frequency generator, the matchbox and the antenna and the plasma.

in operation. The metal pipe that can be seen in figure 3.8 is the implementation of the optimized neutral injection discussed in section 5.4.2 of chapter 5 .

The two large pyrex windows on either side of the chamber are set with a distance from the thruster end panel matching the length of the thruster. The extraction tubes and the region outside, therefore, can be observed to place probes accurately. In future work, these windows can be used for laser based diagnostics on the exiting plasma plane.

\subsubsection{Matchboxes}

The matchbox is an essential part of the power coupling to create a radio-frequency excited plasma. Contrary to direct-current generated plasmas where the power is coupled directly, the fact that the voltage and current are propagating must be taken into account for radio-frequency generated plasmas.

The impedance $(\tilde{Z})$ of a circuit element is a complex value defined as the ratio between the voltage $(\tilde{V})$ across this element and the current $(\tilde{I})$ flowing through this element

$$
\tilde{V}=\tilde{Z} \tilde{I} \quad \text { with } \quad \tilde{Z}=R+j X=Z e^{j \theta},
$$

with the resistance $R$ (impedance in case of a direct-current circuit) and the reactance $X$. The expression $Z e^{j \theta}$ gives directly the ratio $Z$ between $V_{\max }$ and $I_{\max }$, and the phase difference $\theta$ between the voltage and the current. If the impedance changes along the circuit, part of the power is reflected at the interface with a coefficient $\Gamma_{r}$ while the other part is transmitted with $\Gamma_{t}$

$$
\Gamma_{r}=\frac{\tilde{Z}_{2}-\tilde{Z}_{1}}{\tilde{Z}_{1}+\tilde{Z}_{2}} \quad \text { and } \quad \Gamma_{t}=1-\Gamma_{r},
$$

with $\tilde{Z}_{1}$ the impedance of the first part of the circuit and $\tilde{Z}_{2}$ of the second one.

In the case of plasmas, the power generator $\left(\tilde{Z}_{g}\right)$ and the combination of the antenna and the plasma $\left(\tilde{Z}_{p}\right)$ have very different impedances, resulting in little power coupled to the plasma. The matchbox $\tilde{Z}_{m}$ is inserted between the generator and the antenna, with a total impedance $Z_{m, p}$ for the matchbox, the antenna and the plasma. The value of $\tilde{Z_{m}}$ can be adjusted to minimize $\Gamma_{r}$. The matchbox used for the experiments is called a L-circuit matchbox with two high voltage capacitors connected as shown in figure 3.9. 
The capacitor connected in parallel is called the load capacitor $\left(C_{L}\right)$ and the one in series is called the tune capacitor $\left(C_{T}\right)$.

Two sets of variable capacitors were used for experiments:

- first set: $C_{L}=210-500 \mathrm{pF}$ and $C_{T}=620-2600 \mathrm{pF}$

- second set: $C_{L}=12-500 \mathrm{pF}$ and $C_{T}=7-1000 \mathrm{pF}$

It should be noted that in the actual matchboxes, each capacitance is in fact a parallel combination of several capacitors, and the value of the capacitance is the sum of the capacitors involved.

It will be shown in chapter 4 that the matchbox circuit design may also be changed in order to minimize the amount of applied power coupled capacitively to the plasma. 


\section{Chapter 4}

\section{Ionization Stage}

\section{Contents}

4.1 Power Coupling, Symmetry and Stability . . . . . . . . 54

4.1 .1 Helicon Mode . . . . . . . . . . . . . . . . . . . . 54

4.1 .2 Design Evolution . . . . . . . . . . . . . . . 55

4.1 .3 Plasma Asymmetry . . . . . . . . . . . . . . . . 60

4.1 .4 Instabilities . . . . . . . . . . . . . . . . 63

4.2 Positive Ion Current Density . . . . . . . . . . 65

4.2 .1 Original Matchbox Circuit . . . . . . . . . . . . 66

4.2 .2 Modified Matchbox Circuit . . . . . . . . . . . . 67

4.3 Mass Efficiency .................. 70

4.3 .1 Definition . . . . . . . . . . . . . . 70

4.3.2 Non-Uniformity of the Current Density . . . . . . . . . . . . 71

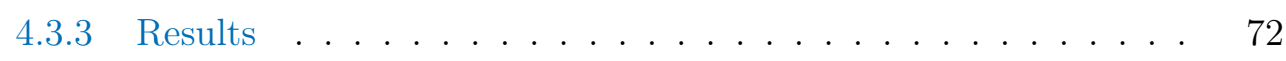

4.4 Electron Temperature ................. 72

4.4 .1 Interest . . . . . . . . . . . . . . . . . 73

4.4 Discussion of the Results . . . . . . . . . . . . . . 74

4.5 Conclusion ...................... 77

4.5 .1 Power Coupling . . . . . . . . . . . . . . . . . . . . . . . . . . . . . . . . . 77

4.5 .2 Positive Ion Current Density _. . . . . . . . . . . 78

4.5 .3 Mass Efficiency . . . . . . . . . . . . . . . . 78

4.5.4 Electron Temperature . . . . . . . . . . . . . 78

The first stage of the PEGASES thruster is the ionization stage where the electric power is applied to the feedstock gas to create and sustain the plasma. The efficiency of the power coupling is crucial in the case of thrusters as the available power in spacecrafts is generally limited. The PEGASES prototype I, as its name suggests, was the first implementation of the PEGASES concept. As experiments were run, they showed that the first design of this prototype could be improved. The PEGASES thruster prototype I thus underwent two evolutions: the antenna was changed, and the matchbox circuit was modified. 
Three aspects of the ionization stage were investigated. First, the positive ion current density was measured in the extraction zone, as the plasma diffuses from the thruster cylinder. Second, the mass efficiency, which is the ratio of ion flux to neutral flux, was evaluated. Finally, as most plasma processes are governed by the electron temperature, the latter was investigated as a function of radius, from the center of the thruster cylinder to the outer edge of the extraction tube.

Although the PEGASES concept is based on electronegative gases, the study was done with argon (electropositive gas) and oxygen (electronegative gas). In a noble gas plasma, mainly two types of charged particles are present (electrons and singly-charged positive ions) which makes the plasma creation and sustainment easier to obtain and understand compared to electronegative plasmas where at least three types of charged particles can be found (electrons, positive and negative ions) leading to different energy couplings and instabilities.

\subsection{Power Coupling, Symmetry and Stability}

In a radio-frequency discharge, three coupling modes are possible: the capacitive, the inductive and the helicon mode (see section 1.1.2). As the plasma densities obtained are highest with the helicon mode, this is what is aimed at for the PEGASES thruster. The properties of this mode are first described.

\subsubsection{Helicon Mode}

In a capacitive discharge, the electrons are heated by the oscillating sheaths. Since sheaths are small regions near the walls, a very small part of the plasma is actually heated. For inductive discharges, the electrons are excited by a penetrating electromagnetic wave. As it is absorbed by the plasma, the heating happens in a small region characterized by the skin depth (characteristic length of an exponential decrease). The helicon mode is different from these two modes in that the electron heating occurs in the whole plasma volume since the helicon wave can propagate anywhere in this volume.

In the presence of a static magnetic field, the plasma becomes anisotropic: charged particles are no longer free to diffuse in any given direction. The diffusion along the magnetic field differs from the diffusion across it. Similarly to the diffusion of charged particles, the propagation of waves is changed from the isotropic case and depends on the direction, along or across the magnetic field. The dispersion relation, therefore, is different in the two cases and requires the definition of a plasma dielectric tensor instead of a plasma dielectric constant to take into account the different directions. The dispersion relation in magnetized plasmas shows that waves with a frequency lower than the electron plasma frequency can propagate in the direction of the magnetic field [47]. These waves can be separated into two categories depending on their polarization. Left-hand polarized waves are called Alfvén waves and right-hand polarized whistlers, among which helicon waves form a sub-family. The role of waves in magnetized plasmas can be traced back to Hartree in 1931 [48] and Alfvén mentioned an application to sun spots in 1942 [49]. As to helicon waves, the name comes from the rotating polarization which results in a helical motion [50].

The polarization describes how the electric field rotates around the magnetic field when looking in the same direction as the magnetic field. Right-hand polarized waves thus 
rotate clockwise and left-hand polarized waves counter-clockwise. As a charged particle rotates around a magnetic field line, a wave rotating in the same direction seems almost constant (and is constant when there is resonance) while the wave rotating in the opposite direction oscillates at an even higher frequency. On average, only the wave rotating in the same direction can accelerate the particle as the average of a sinusoidal oscillation is zero. Since radio-frequency excited plasmas are considered where the ions cannot react to the electromagnetic field, but electrons can (see section 2.1.2), only one polarization is capable of heating the electrons. When looking in the same direction of the magnetic field, an electron has a clockwise motion. Right-hand polarized helicon waves, and not left-hand polarized Alfvén waves, can heat electrons.

Different modes of helicon waves, referenced by their mode number $m$, are possible and describe different electromagnetic patterns in the azimuthal plane as the wave propagates [51]. The mode $m=0$ is the simplest one and possesses point symmetry. This means that it can be excited with simple antenna geometries, such as a single loop. The modes $m= \pm 1$ possess a more complicated azimuthal geometry and require specific antenna designs to be excited. Examples of these designs are the double-saddle antenna (Boswelltype) [11], the Nagoya antenna (type III) [52], and the helical antenna [53]. However, it was shown that only the $m=+1$ mode provides an efficient power coupling. As antennae capable of exciting the $m= \pm 1$ modes can also excite the $m=0$ mode, the total heating is better with these antennas. It should be noted that for $m=1$, the antenna creates a spatially varying electromagnetic field with maxima near the ends of the antenna. This distance results in a specific wavelength for the helicon waves, which means that only harmonics of this fundamental wavelength can be sustained.

Each mode and harmonic of a helicon wave is characterized by a limit density below which it cannot be launched. This means that the plasma has to be created by other coupling modes, such as capacitive and inductive, before the helicon mode can be reached. Once the threshold density is reached, the plasma density is increased by the helicon waves. This is the reason why the plasma density as a function of electrical power is not continuous as different modes are reached and increase the plasma density.

\subsubsection{Design Evolution}

A Boswell-type antenna, shown in figure 4.1 after it was removed, was first used. The power coupling proved very weak. As the capacitors of the matchbox were changed to no avail, the antenna was determined to be the cause of the poor power coupling. It was thus changed for another design. Moreover, aiming at reducing the capacitive coupling, the matchbox circuit was modified.

\section{Boswell-Type Antenna}

A filament probe inserted at the center of the cylinder through the left extraction tube, which is only $7 \mathrm{~cm}$ away from the antenna region, could not measure significant currents. Although very small, the filament probe was enough to extinguish the plasma once inserted in the thruster cylinder. Measurements with a planar probe biased to $-60 \mathrm{~V}$ at the edge between the cylinder and the extraction tube, i.e. at $r=2.9 \mathrm{~cm}$, were done in argon at $10 \mathrm{sccm}$ and $P=200 \mathrm{~W}$ for different values of the magnetic field from 0 to $3 \mathrm{~A}$. The average measured current density was $J \sim 50 \mu \mathrm{Acm}^{-2}$. This very small value of the current density, in addition to the fact that an unbiased filament probe in the middle of 
the plasma extinguishes it, shows a very poor power coupling. Moreover, as the plasma was localized near the antenna, it points to a weak capacitive coupling.

At first, the gas feed was introduced through the matchbox and the cylinder connecting it to the thruster. As a metal wall of the matchbox was replaced with a metal grid, the matchbox was still shielded but could also be looked into. Since the gas feed was a plastic tube placed in-between the two copper rods connecting the antenna to the matchbox, a plasma could be created and would take part of the electrical power from the plasma that should be created in the thruster cylinder. The higher neutral density in the plastic tube would also enhance plasma creation or diffusion into the tube. This proved to be the case as a plasma in the plastic tube was observed as far as $20 \mathrm{~cm}$ from the gas entry in the thruster cylinder. In order to prevent such plasma formation, two steps were taken. The plastic tube was replaced with a metal tube going through the metal cylinder connecting the matchbox to the thruster chamber panel. A grid was placed at the connection to the small pyrex tube to neutralize any particles diffusing upstream.

In order to estimate how much power was coupled to the plasma, and how much was dissipated in the antenna, the following experiment was done at $P=200 \mathrm{~W}$ for $20 \mathrm{sccm}$ in argon. First, with argon flowing in the thruster, the reflected power is minimized by tuning the matchbox. Second, the gas flow is stopped. The difference in reflected power gives an indication of how much power is actually coupled to the plasma. The difference turned out to be a few watts and shows that the antenna could not couple the power to the plasma.

Two L-circuit matchboxes were used with the Boswell-type antenna. The first one consisted of an automated matchbox with capacitor values $C_{L}=210-500 \mathrm{pF}$ and $C_{T}=$ $620-2600 \mathrm{pF}$. The reflected power is measured in real-time and a circuit controlling the two capacitors tries to minimize this reflected power. This optimization in reflected power proved impossible to achieve as the circuit would try to lower the capacitor values to their limit. This behavior shows that the power coupling was definitely not a helicon mode which the matchbox was designed for. As the second matchbox, manually operated with lower capacitor values $C_{L}=12-500 \mathrm{pF}$ and $C_{T}=7-1000 \mathrm{pF}$, showed no improvement, the issue lay elsewhere.

Figure 4.1 shows the Boswell-type antenna after it was removed from the thruster. All support pieces are made of polyfulfone which melts at a temperature higher than $185{ }^{\circ} \mathrm{C}$. In the design of a double-saddle antenna, the copper sheet is wrapped for a quarter of a turn above itself. The polysulfone melted around this region, resulting in a short-circuit. The heating of the antenna is additional proof that the electrical power was not coupled to the plasma but was lost in the antenna itself.

Since this implementation of the Boswell-type antenna proved unable to reach proper plasma densities, results in the following sections and chapters only consider the second antenna (three-coil antenna).

\section{Three-Coil Antenna}

Therefore, the antenna was changed for a simpler design which is optimized for inductive heating, but is also capable of launching helicon waves in three modes $m \in\{-1,0,1\}$. This design is a three-coil antenna wrapped directly around the pyrex cylinder and centered around the small pyrex tube where the gas is introduced, as can be seen in figure 4.2.

The connectors making the junction between the antenna and the copper rods are set above the antenna to avoid short-circuits. Although the design of the antenna is based 


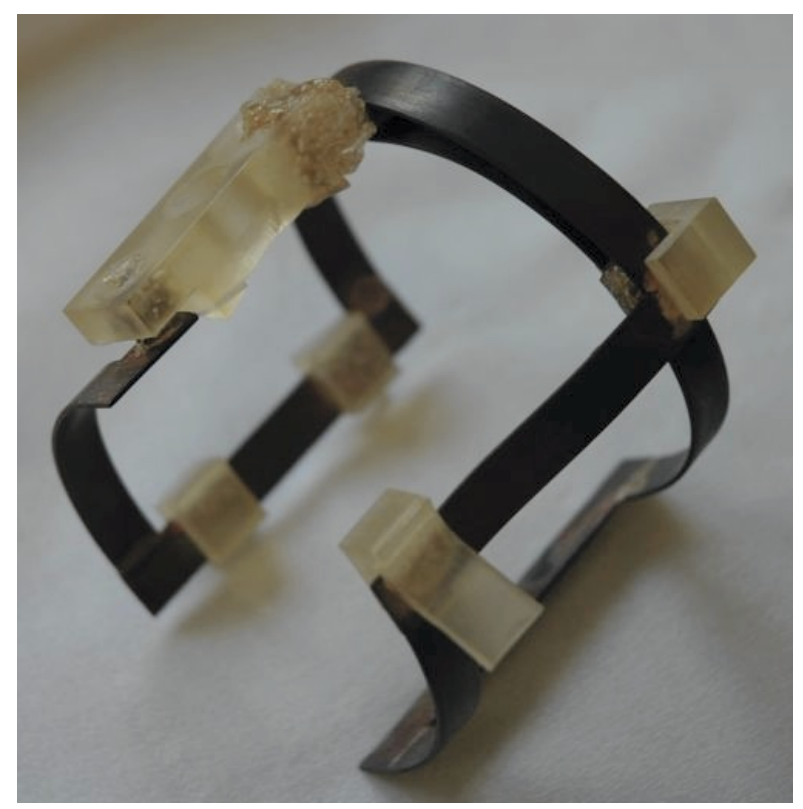

Figure 4.1: Boswell-type (double-saddle) antenna used in the first design of the PEGASES thruster prototype I, after it was removed from the thruster.

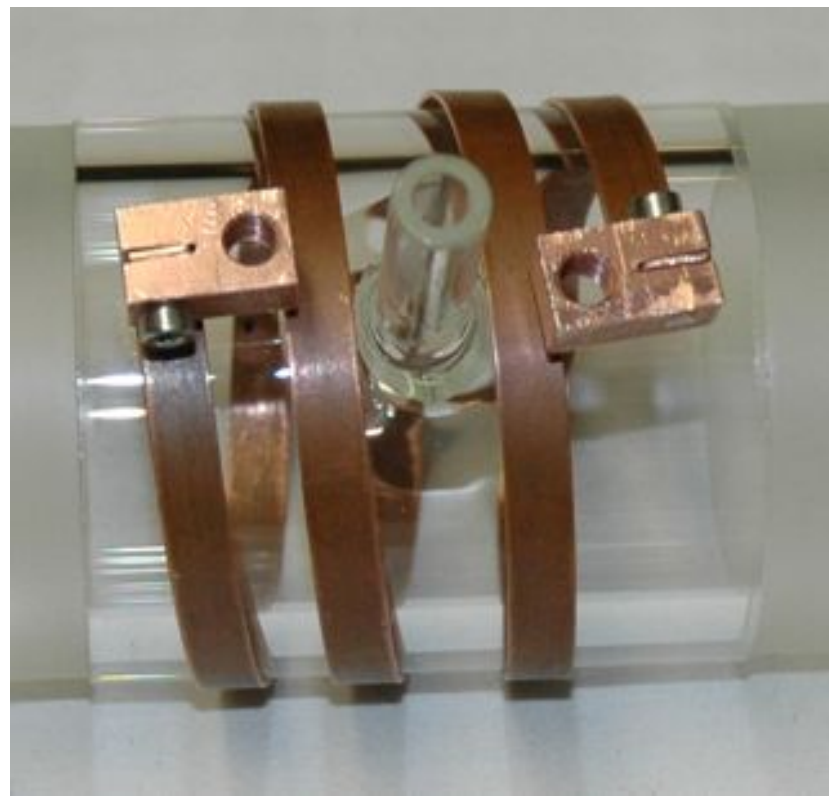

Figure 4.2: Three-coil antenna wrapped around the thruster cylinder and centered around the gas injection. 


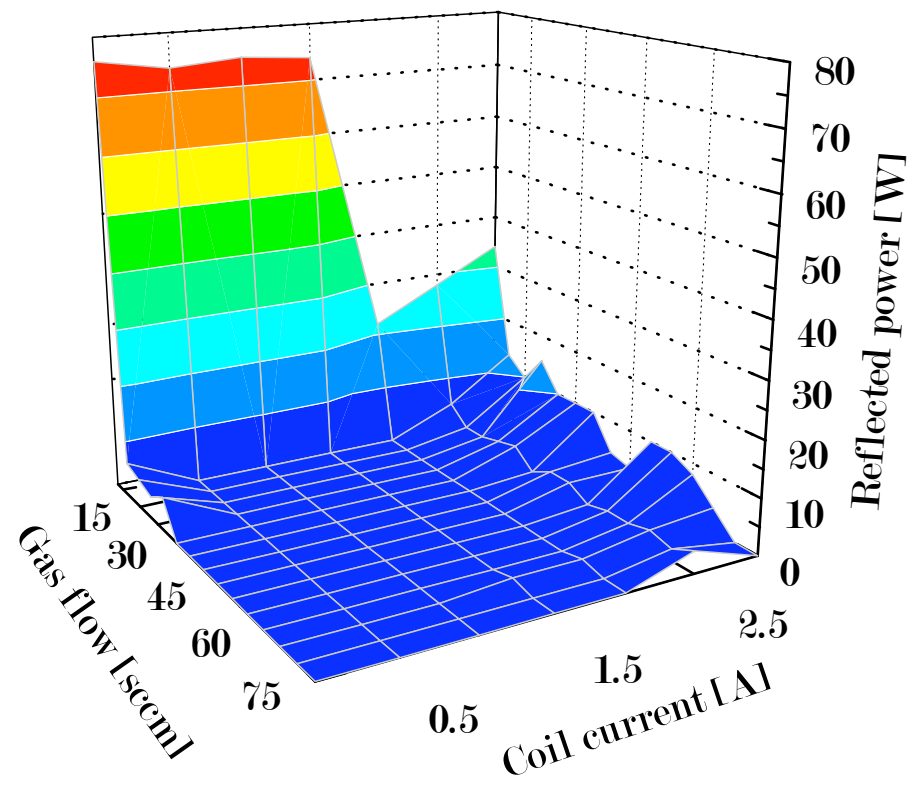

Figure 4.3: Reflected power in $W$ in argon for $P=200 W$. The magnetic field is varied with the coil current from 0 to $3 \mathrm{~A}$, and the gas flow from 5 to $75 \mathrm{sccm}$.

on a loop, which can only launch helicon waves in the $m=0$ mode, the angle between the loops and the plane perpendicular to the magnetic field makes the modes $m= \pm 1$ possible.

The larger the number of turns, the stronger the electromagnetic field that is created in the cylinder. At the same time, high powers require a large enough cross-section for the current to flow in the conductor which results in a large sheet, with turns that must be kept apart to avoid short-circuits. Three turns were chosen as a compromise between electromagnetic field strength and preventing short-circuits.

Experiments in argon for the same flow $(10 \mathrm{sccm})$ and power $(200 \mathrm{~W})$ as for the first antenna showed a dramatic improvement in power coupling. The experiment with a planar probe placed at the cylinder and extraction tube edge, and biased to $-60 \mathrm{~V}$ was redone. The current density was measured to be around $J \sim 50 \mathrm{mAcm}^{-2}$. This corresponds to a factor of $10^{3}$ increase.

To characterize the power coupling with the three-coil antenna, figure 4.3 shows the reflected power in argon for an applied power of $P=200 \mathrm{~W}$, as a function of magnetic field for a coil current from 0 to $3 \mathrm{~A}$ and gas flow from 5 to $75 \mathrm{sccm}$. It can be seen that the reflected power is negligible over most of the parameter space. The applied power, therefore, is coupled properly to the matchbox and the antenna, except at very low pressure $(5 \mathrm{sccm})$. The influence of the magnetic field, moreover, can only be seen at high current $(I=3 \mathrm{~A})$, with a small increase in reflected power.

\section{Modified Matchbox Circuit}

The circuits of the two matchboxes were classical L-circuits, with a load capacitor and a tune capacitor (see section 3.3.2). In such a matchbox circuit, one of the antenna ends is connected directly to the ground, meaning that whatever the incoming voltage or current is, the potential at this end remains zero. The L-circuit and the corresponding voltage distribution are shown in figure $4.4 \mathrm{a}$. The voltage across the antenna is highly asymmetrical 


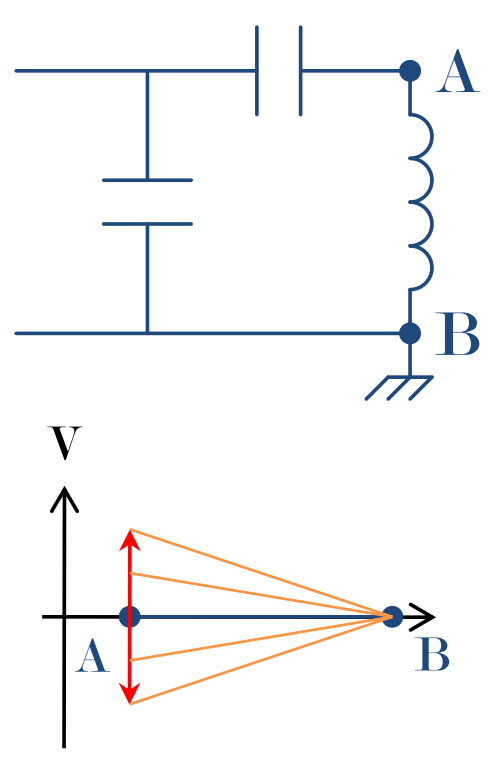

(a) L-circuit
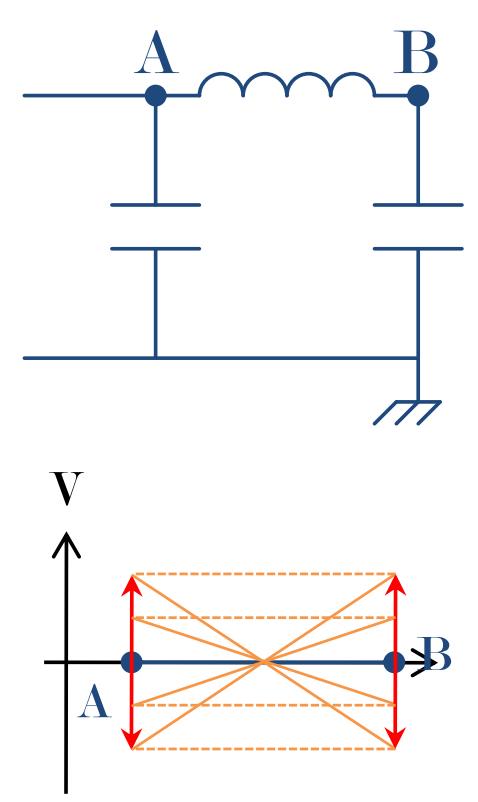

(b) П-circuit

Figure 4.4: Matchbox circuit and voltage across the antenna for the L-circuit (a) and the new design (b). The voltage across the antenna for the new design is shown in the symmetrical case where the voltage amplitude is the same at both antenna ends for two cases: opposite-phase (solid line) and in-phase (dotted line).

with most of the capacitive power deposition near one end of the antenna. This induces large potential fluctuations in the plasma and makes probe diagnostics problematic. In addition, it also induces a very large sputtering rate of the thruster cylinder near the high voltage end of the antenna.

The new matchbox circuit corresponds to a $\Pi$-circuit, where the tune capacitor and the antenna have switched positions from an L-circuit. Neither of the antenna ends is now grounded. The voltage across the antenna now depends on the phase difference between the two ends, which itself depends on the impedance of the antenna and the capacitor (LC-circuit). The two simple cases of opposite-phase (solid line) and in-phase (dotted line) are shown in figure $4.4 \mathrm{~b}$. Since the antenna length $(\sim 60 \mathrm{~cm})$ is much smaller than the characteristic wavelength of the excitation at $13.56 \mathrm{MHz}(\sim 22 \mathrm{~m})$, there are no additional oscillations in the antenna. For the in-phase case, the voltage is thus constant over the antenna. As there is no potential difference across the antenna, it consists of a capacitive coupling and, therefore, should be avoided. In the opposite-phase case, one can see a zero voltage in the middle of the antenna: the voltage distribution corresponds to two antennas with the previous matchbox circuit. However, the amplitude of the oscillation on each end of the antenna is half of the previous amplitude (L-circuit), since the voltage is now distributed over the antenna. The fluctuation and sputtering issues, therefore, are reduced; it is the better case. However, these two cases are purely theoretical.

The actual voltage amplitudes were measured with a point-to-point high voltage probe in argon for $30 \mathrm{sccm}$ at $P=100 \mathrm{~W}$ with two magnetic field values with coil currents 0 and $1 \mathrm{~A}$. The gas flow was chosen at $30 \mathrm{sccm}$, a value with a proper power coupling. The power and magnetic field strength, however, were chosen lower than the optimum in order to keep the voltages at the antenna ends low. The voltages are summarized in table 4.1. 


\begin{tabular}{ccc}
\hline $\mathrm{B}[\mathrm{A}]$ & $C_{L} \operatorname{tip}[\mathrm{V}]$ & $C_{T} \operatorname{tip}[\mathrm{V}]$ \\
\hline \hline 0 & 250 & 1530 \\
1 & 125 & 1000 \\
\hline
\end{tabular}

Table 4.1: Voltages on both tips of the antenna in argon for $30 \mathrm{sccm}$ and $100 \mathrm{~W}$. Two magnetic field strengths are considered with coil currents 0 and $1 \mathrm{~A}$.

It can be seen that the voltage across the antenna does not correspond to the oppositephase case previously discussed, but is almost the reverse of the voltage distribution for an L-circuit: the voltage amplitude of the tip connected to the load capacitor is very small compared to that of the other tip (tune capacitor).

For the modified matchbox circuit, the influence of the applied power is investigated in figure $4.5 \mathrm{in}$ argon. The reflected power as a function of magnetic field, coil current from -3 to $3 \mathrm{~A}$, and gas flow, from 5 to $80 \mathrm{sccm}$, is shown for $P=100 \mathrm{~W}$ (figure 4.5a) and $P=300 \mathrm{~W}$ (figure $4.5 \mathrm{~b}$ ). It can be seen that the applied power cannot be properly coupled to the matchbox and the antenna at low pressure, below $20 \mathrm{sccm}$, whether at 100 or $300 \mathrm{~W}$. For these gas flows, the neutral and plasma densities are too low for the design of the matchbox circuit. At low power, values of the magnetic field higher than a coil current of $2 \mathrm{~A}$, whether positive or negative, prevent a proper power coupling. At high power, the magnetic field has little effect on the reflected power as it stays below $20 \mathrm{~W}$, which corresponds to $7 \%$.

The influence of the feedstock gas (modified matchbox) is shown in figure 4.6 where the reflected power can be seen at $P=300 \mathrm{~W}$ for argon (figure 4.6a) and oxygen (figure 4.6b) as a function of magnetic field, coil current from -3 to $3 \mathrm{~A}$, and gas flow, from 5 to $80 \mathrm{sccm}$. The first important feature is the fact that a plasma cannot be obtained for $5 \mathrm{sccm}$ in oxygen. Moreover, the reflected power quickly becomes comparable to the applied power for a magnetic field above a coil current of $2 \mathrm{~A}$, independently of direction. The power coupling, therefore, is weaker in oxygen than in argon, and will be further investigated in section 4.2.

Before its modification, the matchbox circuit consisted of grounding one end of the antenna. The resulting alternating voltage across the antenna was thus highly asymmetrical, as was discussed in the previous section for the first antenna. By switching the antenna and the tune capacitor, the circuit of the modified second matchbox aimed at symmetrizing the alternating voltage across the antenna. This was clearly not achieved as the point-to-point voltage measurements on the antenna showed that the ratio between the voltage amplitude on both ends of the antenna could be as high as 8 (magnetic field with a coil current of $1 \mathrm{~A}$ in table 4.1).

\subsubsection{Plasma Asymmetry}

The first prototype of the PEGASES thruster was built as a double helicon in order to keep the issue of ion acceleration as open as possible: positive ions could be ejected from one extraction tube and negative ions from the other one, for instance. For such a scenario, the plasma creation should be symmetrical for the ejected plasma to be overall neutral. In the other case of both ion species being ejected from each extraction tube, symmetry is also required to prevent any rotational effect: as the thrust is greater on one side, the thruster and the body to which it is attached have a rotation motion. The three-coil antenna setup of the PEGASES thruster prototype, however, was characterized 


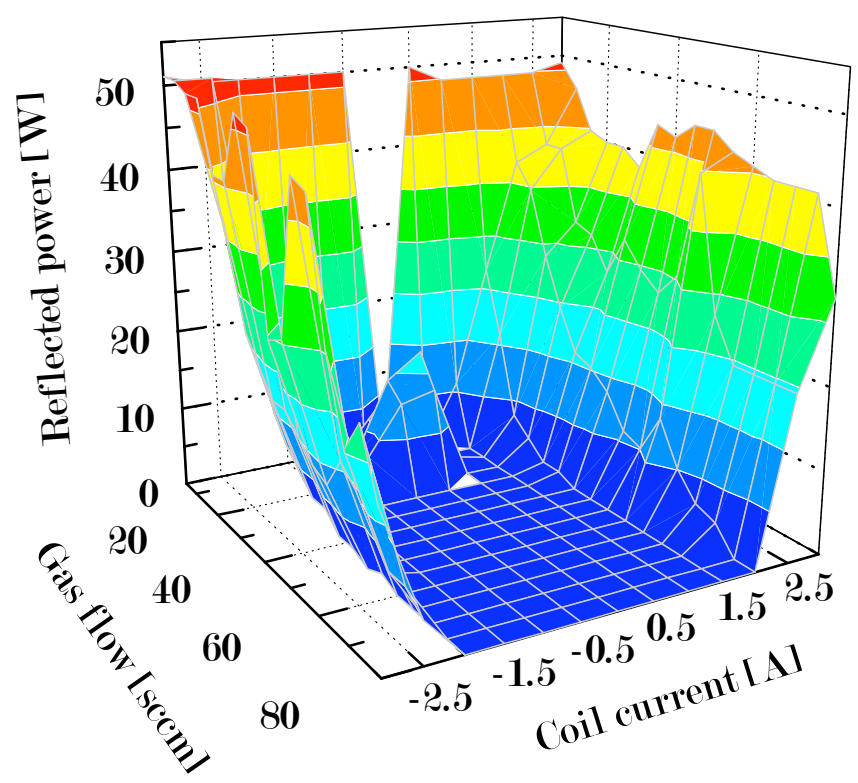

(a) $100 \mathrm{~W}$

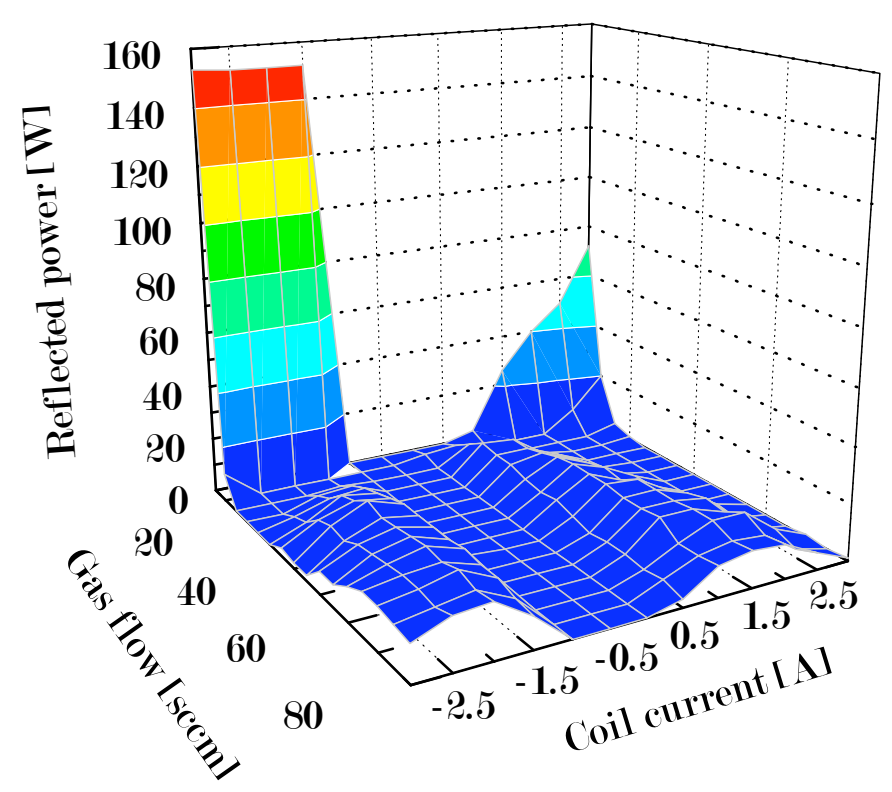

(b) $300 \mathrm{~W}$

Figure 4.5: Reflected power in $W$ in argon for $P=100 W(a)$ and $P=300 W$ (b). The magnetic field is varied with the coil current from -3 to $3 \mathrm{~A}$, and the gas flow from 5 to 80 sccm. 


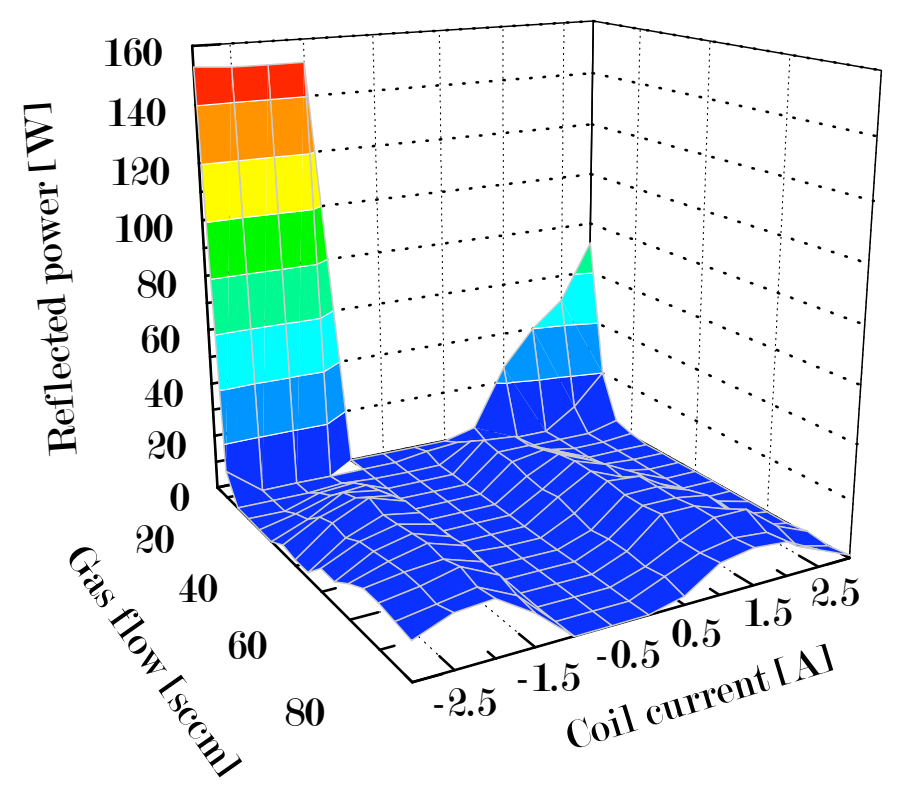

(a) $A r$

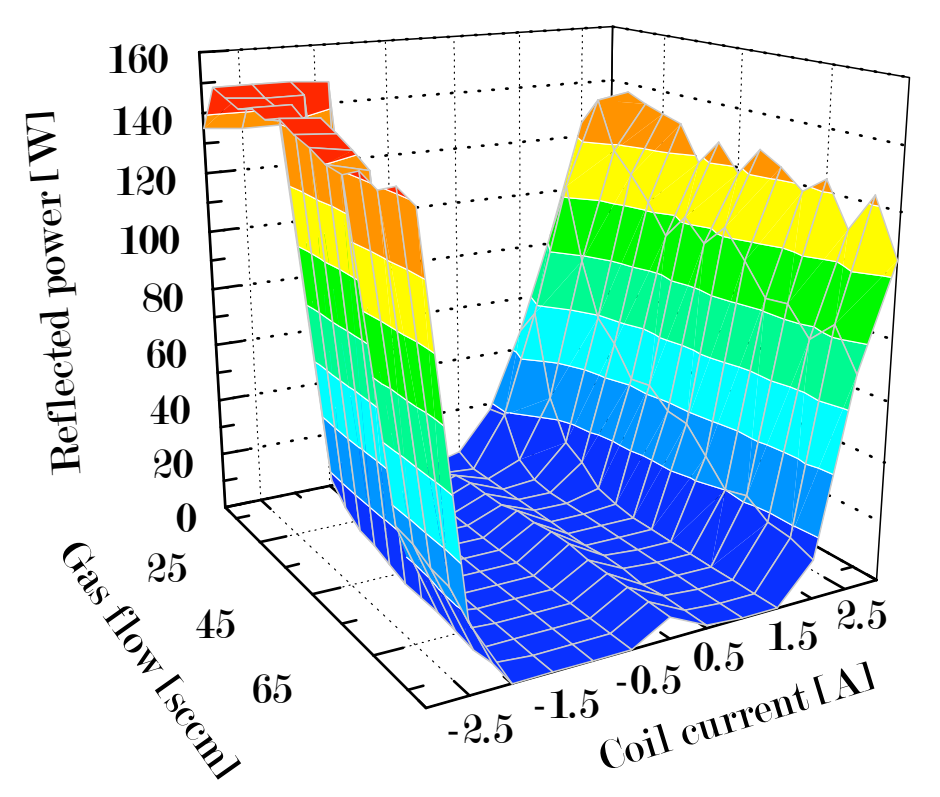

(b) $\mathrm{O}_{2}$

Figure 4.6: Reflected power in $W$ in argon (a) and oxygen (b) for $P=300 W$. The gas flow is varied from 5 to $80 \mathrm{sccm}$ in argon, and from 10 to $80 \mathrm{sccm}$ in oxygen; the magnetic field with the coil current from -3 to $3 \mathrm{~A}$. 
by a plasma creation asymmetry.

As can be seen in figure 3.4 in section 3.2.1, the magnetic field is not constant over the length of the thruster cylinder, with an especially strong gradient in the source region between the two central coils. With a weaker magnetic field, the field lines diverge outward from the axis of the thruster cylinder. Although the magnetic field lines are roughly symmetrical with respect to the center of the thruster cylinder (middle point on the axis), the gradient in the source region plays an important role, especially since the antenna is asymmetrical. In the case of capacitive and inductive modes, the divergence only affects the plasma diffusion, which can lead to asymmetries. For the helicon mode, however, the divergence plays a greater role, since the helicon waves propagate preferably along the magnetic field lines.

In cases where the asymmetry was stable, the power coupling mode was seen to play a role in whether the brighter extraction tube was the left or right hand side one. This effect was seen as the magnetic field strength was varied with the coil current from 0 to $3 \mathrm{~A}$. Without magnetic field, the discharge was inductive. For small values of the magnetic field, the discharge was still inductive (usually called magnetized inductive). The right hand side extraction would be brighter, meaning a higher plasma density. As soon as the helicon mode was reached for a high enough magnetic field, corresponding to a step in plasma brightness and density, the left hand side extraction tube would become the brighter one. The plasma creation and diffusion, therefore, were clearly dependent on the power coupling mode.

As the copper sheet was curved by hand to obtain the three-coil antenna, the space between two turns could not be made constant. The radio-frequency excitation, therefore, was slightly asymmetrical, which is enhanced by the diverging magnetic field lines. In capacitive or inductive mode, the plasma diffusion is changed by the magnetic field line divergence, which could lead to a higher plasma density in one side of the thruster cylinder. As plasma densities much higher than the first antenna were reached, helicon waves were launched and propagated in the thruster cylinder. The propagation of helicon waves, as described in section 4.1.1, occurs mostly along the magnetic field lines. The strong divergence in the source region means that the helicon waves cannot propagate in the whole thruster cylinder. The electron heating is thus stronger in some parts of the thruster cylinder.

For all the parameters investigated for the second antenna, whether the matchbox circuit was modified or not, the plasma density was always higher on one side of the thruster cylinder, resulting in a higher plasma density in one of the extraction tubes. Interestingly, the higher plasma density could be in either side of the thruster cylinder, whether steady or oscillating between the two sides. As the plasma densities were much greater than for the first antenna, the difference in light intensity was less obvious. Depending on the parameters, a color difference could still be seen, indicating different electron temperatures. This confirms that the electron heating was not symmetrical.

\subsubsection{Instabilities}

The stability of the plasma is an important factor for a thruster. As the flux of exiting ions depends on the plasma density, fluctuations in plasma density lead to fluctuations in thrust (outgoing ion flux). 


\section{Frequency Spectrum}

For all experiments, the frequency $f=50 \mathrm{~Hz}$ could be observed. However, this is not an oscillation in the plasma due to the plasma itself or the matchbox circuit. The cause of this oscillation is the direct-current generator used to bias the probes, whether filament or planar. In France, the electrical network uses a frequency of $50 \mathrm{~Hz}$, which the generator could not properly damp.

As the plasma was excited at $f=13.56 \mathrm{MHz}$, this frequency was obviously present in the plasma. Moreover, a plasma is a non-linear object, meaning that higher harmonics of the fundamental frequency can be created. Fast Fourier transforms of the collected current signal on an oscilloscope showed that the second harmonic at $f=27.12 \mathrm{MHz}$ was usually strong, but the third harmonic at $f=40.68 \mathrm{MHz}$ could not be detected.

At first, the plasmas proved to be extremely unstable. The cause was found out to be the mass flow that was perturbed by the electromagnetic field from the antenna.

Some very low frequency behaviors could be observed in the plasma. The oscillation of the brighter side (higher plasma density), described above, was found to occur with frequencies between $0.1 \mathrm{~Hz}$ and a few tens of $\mathrm{Hz}$. Also, the plasma was seen to be flickering in some cases. At constant plasma conditions, the plasma would turn itself on and off at frequencies between $1 \mathrm{~Hz}$ and a few tens of $\mathrm{Hz}$.

An additional frequency around $1 \mathrm{kHz}$ was usually found in the plasma. The actual frequency could vary between $500 \mathrm{~Hz}$ and $4 \mathrm{kHz}$.

\section{Mass Flow Instability}

The first few plasmas created with the three-coil antenna were extremely unstable. However, this was not due to the plasma itself or an interaction between the matching circuit and the plasma, but to the mass flow regulating the gas flow. As it was unshielded, the electromagnetic field created by the antenna perturbed the control on the gas flow. If the gas flow was set at $20 \mathrm{sccm}$ for instance, the pressure measurements with the baratron showed that the gas flow could oscillate between $0 \mathrm{sccm}$ and $40 \mathrm{sccm}$. These extrema of course depend on the choice of applied power and how close the mass flow was to the thruster chamber. The mass flow was thus changed for a shielded one. From this point on, the gas flow in the thruster was steady and matched the set value, proving that the issue was indeed coming from the unshielded mass flow.

It should be noted that the issue of the unshielded mass flow never came up with the Boswell-type antenna, whether the first or second matchbox was used. This is additional proof that the electrical power applied to the implementation of the Boswell-type antenna was not properly converted into an electromagnetic field which in turn would excite the plasma.

\section{Asymmetry Oscillation}

It was mentioned previously that one of the extraction tubes was always brighter than the other. Sometimes, this difference in brightness would be accompanied by a color difference, meaning that the electron temperatures were different. In most cases, the asymmetry was oscillating. This means that the extraction tube showing a higher plasma density or electron temperature would change between the left and right hand sides.

Although the dependence on the parameters could not be determined, the frequency of the oscillation changed with the gas (whether argon or oxygen), the gas flow, the applied 
power and the magnetic field strength. The frequency of this oscillation was found to range from values as low as $0.1 \mathrm{~Hz}$, meaning that the reversal of the high plasma density could take 10 seconds, to values higher than $10 \mathrm{~Hz}$, which the naked eye cannot properly resolve.

The asymmetry oscillations, moreover, were far from regular in most cases. The first irregularity was the fact that at constant parameters, the frequency was not constant, but would vary around an average frequency. This makes it difficult to interpret these oscillations in terms of energy coupling or plasma diffusion altered by the geometry since the oscillation frequency appeared to be random. The duty cycle of these oscillations was also irregular. For instance, the plasma density could be higher in the left hand side extraction tube for $90 \%$ percent of the time, but this value could become $10 \%$ as the plasma was sustained without changing any parameter.

It should be noted that for similar frequencies, from $1 \mathrm{~Hz}$ to a few tens of $\mathrm{Hz}$, the plasma was found to be flickering (turning itself on and off) at constant plasma conditions. This behavior could not be explained.

\section{Plasma Instability}

A plasma instability was found around $f=1 \mathrm{kHz}$, from $500 \mathrm{~Hz}$ to $4 \mathrm{kHz}$. A possible explanation for this plasma instability could be the neutral density variations in the thruster volume. The gas is introduced at the center of the thruster cylinder and is pumped out of the thruster through the extraction tubes. Two effects occur simultaneously with the neutral density. The first one is the distribution of the neutrals between the two sides of the thruster which may vary and not be symmetrical. Although the thruster design is symmetrical when it comes to the gas injection, the gas distribution cannot be controlled. The second effect is due to neutral depletion $[54,55,56,57,58]$. The two main processes for the plasma creation, ionization and attachment, depend on the neutral density. In the low pressure case of the thruster, this density cannot be assumed constant as the plasma density is not negligible compared to the neutral density. Before the plasma is created, the neutral density reaches a steady-state between the gas injection and the gas pumping. As soon as the plasma is created, neutrals are turned into ions and electrons, decreasing the neutral density. With a decreased neutral density, the source terms for the plasma sustainment decrease, leading to a lower plasma density. Since less neutrals are turned into ions and electrons, the neutral density increases, resulting in higher source terms and higher plasma density. This cycle is due to the low pressure and the small residence time of neutrals in the thruster volume.

\subsection{Positive Ion Current Density}

Having described the different evolutions of the PEGASES thruster prototype I to obtain proper plasma densities, results are now shown concerning the performance of the threecoil antenna in terms of ionization efficiency. Measurements of the positive ion flux in the extraction tube are first considered. As the PEGASES thruster concept relies on the cross-field diffusion of ions (see section 1.2.1), understanding the evolution of the current density measured in the extraction tube as a function of plasma parameters (gas flow, magnetic field, etc.) is crucial. The positive ion current density, therefore, is investigated. The measurements are done with a planar probe placed at the center of the extraction 


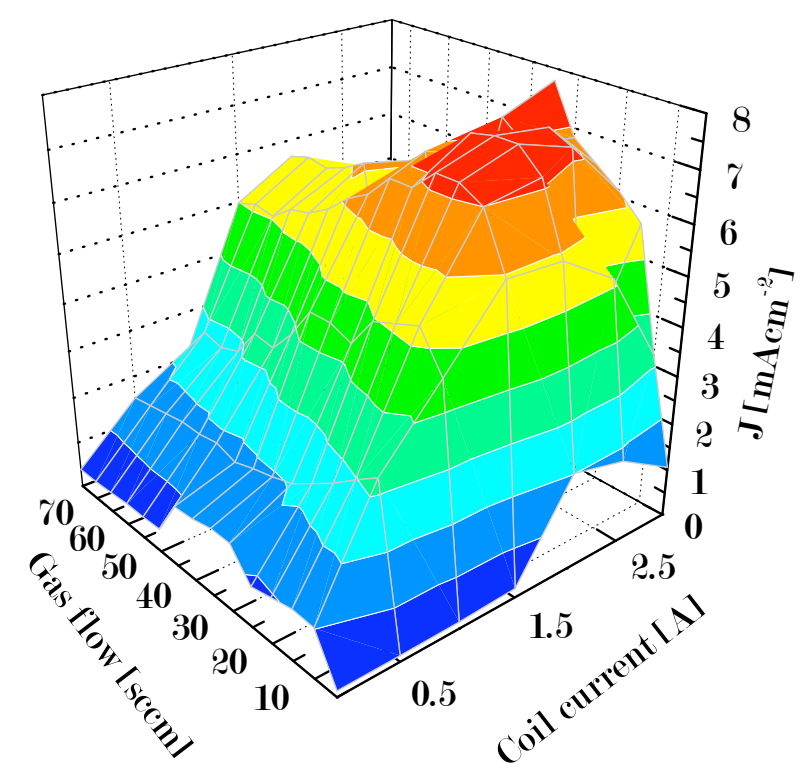

Figure 4.7: Current density in $\mathrm{mAcm}^{-2}$ in argon for $P=200 \mathrm{~W}$. The magnetic field is varied with the coil current from 0 to $3 A$, and the gas flow from 5 to $75 \mathrm{sccm}$.

tube, and at a certain distance from the thruster cylinder. It is biased to $-60 \mathrm{~V}$ to guarantee a measure of the positive ion saturation current (see section 2.2.1).

Preliminary results are presented with the original matchbox configuration. A more complete study is then shown with the modified matchbox circuit.

\subsubsection{Original Matchbox Circuit}

For a fixed electrical power of $200 \mathrm{~W}$ in argon, the current density $J$ in $\mathrm{mAcm}^{-2}$ is measured as two parameters are varied: the gas flow from 5 to $75 \mathrm{sccm}$ and the magnetic field strength with the coil current from 1 to $3 \mathrm{~A}$. The planar probe was placed at the edge between the thruster cylinder and the extraction tube. The resulting surface is shown in figure 4.7. The maximum current density of $J=7.5 \mathrm{mAcm}^{-2}$ is obtained for a gas flow of $25 \mathrm{sccm}$ and a magnetic field with a coil current of $2 \mathrm{~A}$.

Surprisingly, the maximum of current density is not obtained for the maximum value of gas flow, but between 20 and $40 \mathrm{sccm}$. As the gas flow is increased, there are two effects on the plasma density. The total number of neutrals is increased which should lead to an increased plasma density, and therefore current density. However, the edge-to-center density ratio $h$ is a decreasing function of pressure, which means a proportionally lower current density at the edge. Another decreasing effect could come from the electronpositive ion recombination which scales as the square of the plasma density $\left(K_{\mathrm{rec}} n_{e} n_{+}\right)$. As a result, the current density first increases then decreases as a function of the gas flow.

As the magnetic field is increased, the confinement of the electrons also increases. However, argon is an electropositive gas, with electrons and positive ions. Since quasineutrality needs to be fulfilled, the diffusion is ambipolar (same diffusion for electrons and positive ions). As a result, the plasma confinement is an increasing function of the magnetic field. The effect of this increase is twofold. First, the plasma density at the center increases as the loss of electrons and positive ions is reduced by the magnetic confinement. Second, the ratio of edge to center density decreases as the magnetic confinement increases (impeded plasma diffusion). These two effects of the magnetic field cause the current 
density to increase until a maximum is reached for a coil current between 1.5 and $2.5 \mathrm{~A}$, then to decrease.

\subsubsection{Modified Matchbox Circuit}

Contrary to the study before the matchbox circuit was modified, the planar probe is placed near the outer edge of the extraction tube. This was done to investigate the current density near the position where the accelerating grids will be placed in future experiments. Consequently, absolute values of the current density $J$ may not be easily compared with the previous section.

\section{Power Effect}

The influence of the applied power on the current density in argon is shown in figure 4.8 with $100 \mathrm{~W}$ (figure 4.8a) and $300 \mathrm{~W}$ (figure 4.8b). The magnetic field is varied with the coil current from -3 to $3 \mathrm{~A}$, and the gas flow from 5 to $80 \mathrm{sccm}$.

The first effect of the increase in applied power that can be seen is the increase by a factor of 4 of most of the current density values. Moreover, at zero magnetic field, the current density that is negligible compared to the maximum current density at $P=$ $100 \mathrm{~W}$ (ratio of 1 to 20 on average), becomes a fourth of the maximum current density at $P=300 \mathrm{~W}$ (0.5 compared to 2.0). This can be explained by the fact that at $P=100 \mathrm{~W}$ and without magnetic field, the inductive mode is not achieved. The low current density is produced by an inefficient capacitive coupling. At $P=300 \mathrm{~W}$, however, the inductive mode is achieved and results in a current density higher than that of the capacitive coupling. The transition between the capacitive and the inductive mode can thus be seen at zero magnetic field.

Another effect of the applied power increase can be seen in the shift of the maximum current density as function of magnetic field. At $P=100 \mathrm{~W}$, the maximum current density is obtained for a coil current $I= \pm 1.5$ A. At $P=300 \mathrm{~W}$, however, it is reached for $I= \pm 2$ A.

\section{Gas Effect}

The current density as function of magnetic field, with a coil current from -3 to $3 \mathrm{~A}$, and gas flow, from 5 to $80 \mathrm{sccm}$, is shown in figure 4.9 for argon (figure 4.9a) and oxygen (figure $4.9 \mathrm{~b}$ ). It should be noted that a plasma could not be struck for a gas flow of $5 \mathrm{sccm}$ in oxygen, as discussed in section 4.1.2.

The current density profiles are similar, in that they both show two maxima of current density as a function of gas flow and magnetic field. The effect of the two parameters for the two gases is the same as discussed in the previous section. However, two main differences can be seen between argon and oxygen: (i) the current density in oxygen is roughly $\frac{3}{4}$ of the current density in argon, and (ii) the current density goes down to zero in oxygen for high values of the magnetic field. The first difference comes from the fact that oxygen is a molecular electronegative gas, while argon is an atomic electropositive gas. The collisional energy lost per electron-ion pair is greater in oxygen because of additional processes: excitation of vibrational and rotational energy levels, molecular dissociation, and negative ion formation. Depending on the electron temperature, this energy lost per electron-ion pair created in oxygen can be as much as ten times greater than in argon [7]. Consequently, the plasma density is smaller at fixed power. The second difference is 


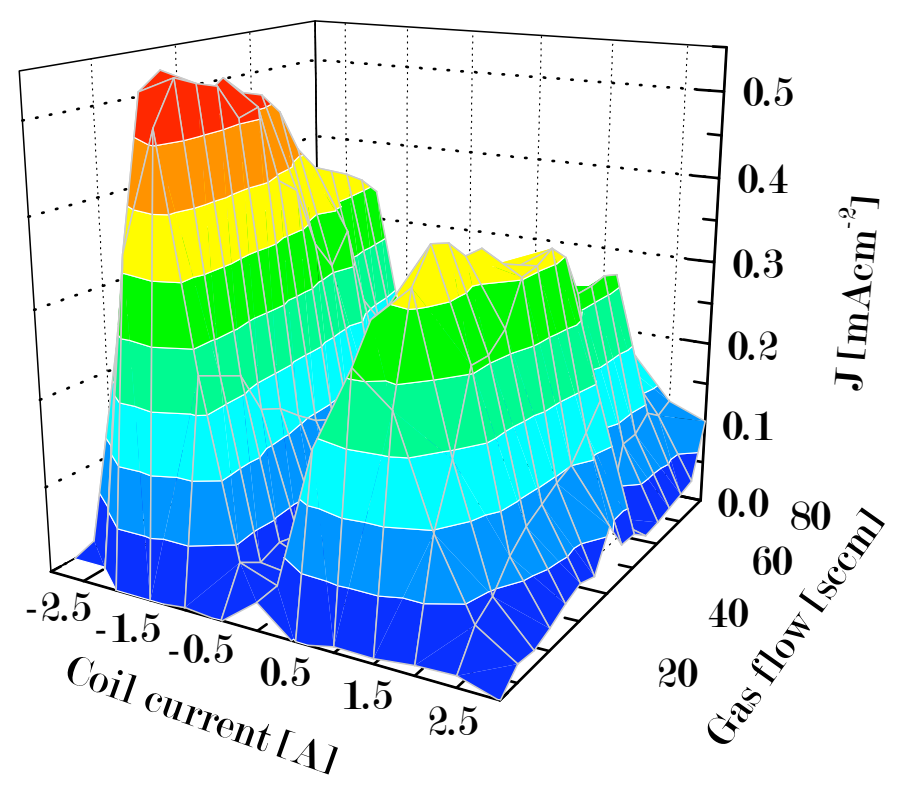

(a) $100 \mathrm{~W}$

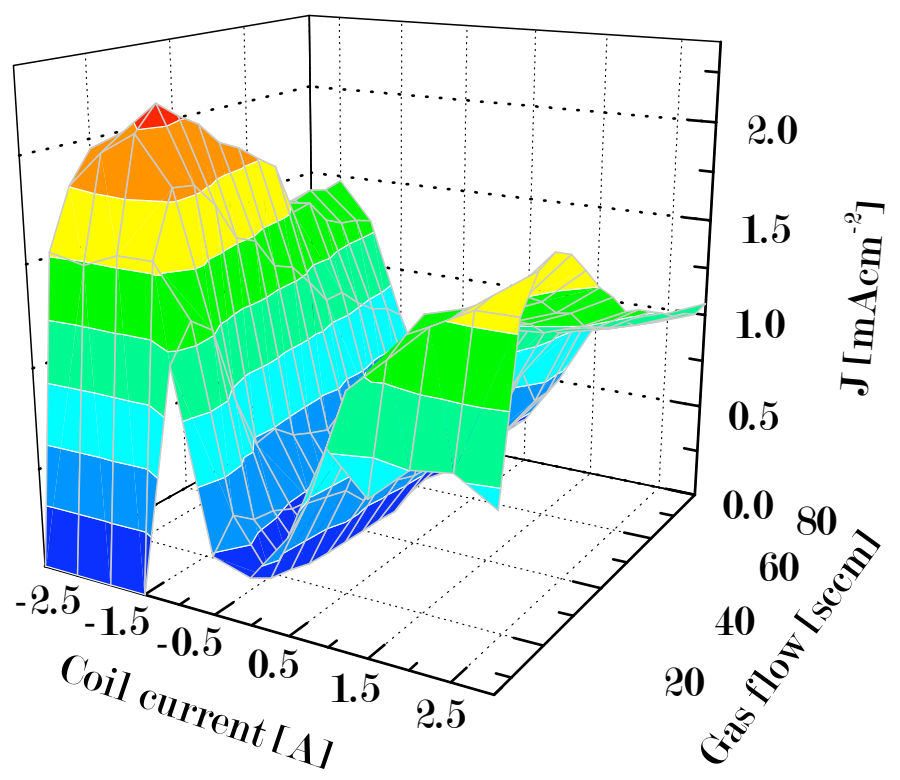

(b) $300 \mathrm{~W}$

Figure 4.8: Current density in $\mathrm{mAcm}^{-2}$ in argon for $P=100 \mathrm{~W}$ (a) and $P=$ $300 W(b)$. The magnetic field is varied with the coil current from -3 to $3 \mathrm{~A}$, and the gas flow from 5 to $80 \mathrm{sccm}$. 


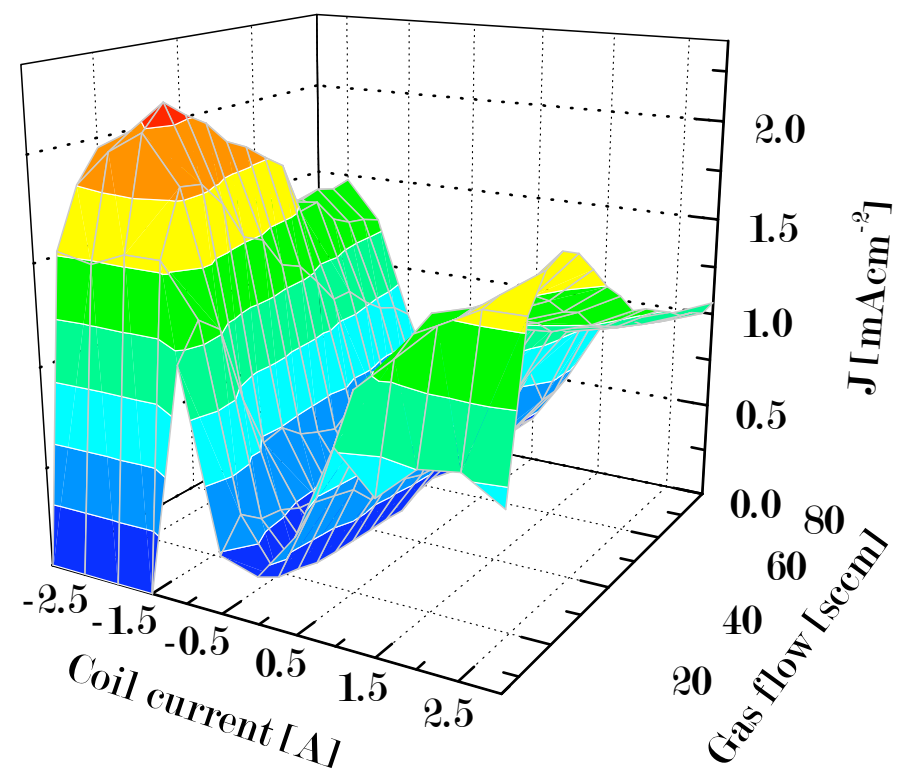

(a) $\mathrm{Ar}$

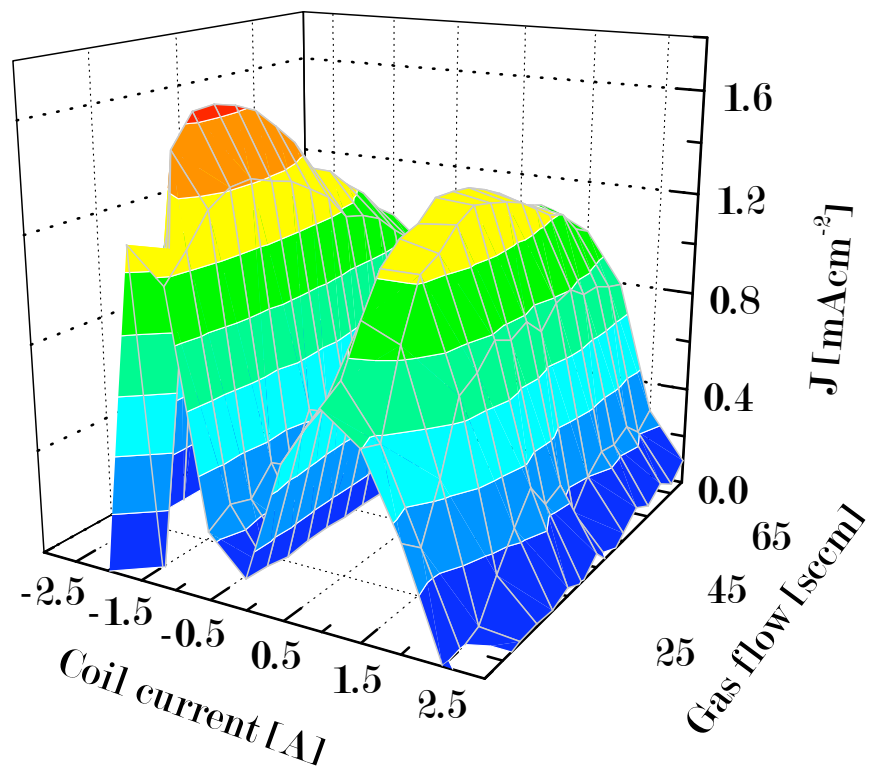

(b) $\mathrm{O}_{2}$

Figure 4.9: Current density in $\mathrm{mAcm}^{-2}$ for $P=300 \mathrm{~W}$ as a function of magnetic field strength (coil current $I \in[-3,3]$ A) and gas flow, which is varied from 5 to $80 \mathrm{sccm}$ in argon (a) and from 10 to $80 \mathrm{sccm}$ in oxygen (a). 
simply due to the very bad power coupling (matching conditions) for oxygen with a coil current of $3 \mathrm{~A}$, as was seen in the reflected power in figure 4.6. For better efficiency in oxygen, the matchbox design should be changed.

\subsection{Mass Efficiency}

Although the evaluation of the mass efficiency is not easy (see below), it is still a good indication of how efficient the thruster will be. The mass efficiency as a function of power was only measured with the three-coil antenna. No measurements were done as the matchbox circuit was modified, since it had little effect on the plasma equilibrium. Another measurement of mass efficiency is presented in the design improvements in section 5.4.1 fo chapter 5 .

\subsubsection{Definition}

In an ideal situation, every single neutral introduced into the thruster is turned into an ion which will provide thrust once accelerated. However, this is not the case as some neutrals are not ionized and exit the thruster unaccelerated. In order to quantify how efficient the thruster is, the mass efficiency is defined as the ratio of positive ion to neutral flux exiting the thruster

$$
M_{\text {eff }}=\left.\frac{\Gamma_{+}}{\Gamma_{\text {neutral }}}\right|_{\text {thruster exit }},
$$

with the neutral flux obtained from the gas flow.

Since there is no gas loss between the entry point and the extraction regions, the neutral flux is conserved between these two points. The neutral flux at the thruster exit can thus be calculated from the gas flow entering the thruster. The gas flow is given in standard cubic centimeter per minute (sccm). The standard conditions of temperature and pressure are $T=0{ }^{\circ} \mathrm{C}$ and $p=101.325 \mathrm{kPa}$. Using the molar volume for these conditions $\mathcal{V}_{M}=22.414 \mathrm{Lmol}^{-1}$, the particle flow for $x \mathrm{sccm}$ is

$$
\text { flow }=\frac{\mathcal{N}_{A} \times x}{\mathcal{V}_{M} \times 60 \times 10^{3}} \mathrm{~s}^{-1}
$$

with $\mathcal{N}_{A}$ the Avogadro constant. The flux is obtained by dividing the flow by the exit area $S$

$$
\Gamma_{\text {neutral }}=\frac{\mathcal{N}_{A} \times x}{\mathcal{V}_{M} \times S \times 60 \times 10^{3}} \mathrm{~m}^{-2} \mathrm{~s}^{-1} .
$$

The neutral flux as a function of gas flow in sccm and exit area in $\mathrm{cm}^{2}$ is thus

$$
\Gamma_{\text {neutral }}=4.48 \times 10^{17} \times \frac{x}{S} \mathrm{~cm}^{-2} \mathrm{~s}^{-1} .
$$

The exit area of the PEGASES thruster prototype is composed of two extraction tubes with a rectangular cross-section $4 \times 4.4 \mathrm{~cm}$, which makes an area of $35.2 \mathrm{~cm}^{2}$. The neutral flux as a function of gas flow in sccm is

$$
\Gamma_{\text {neutral }}=1.27 \times 10^{16} \times x \mathrm{~cm}^{-2} \mathrm{~s}^{-1}
$$




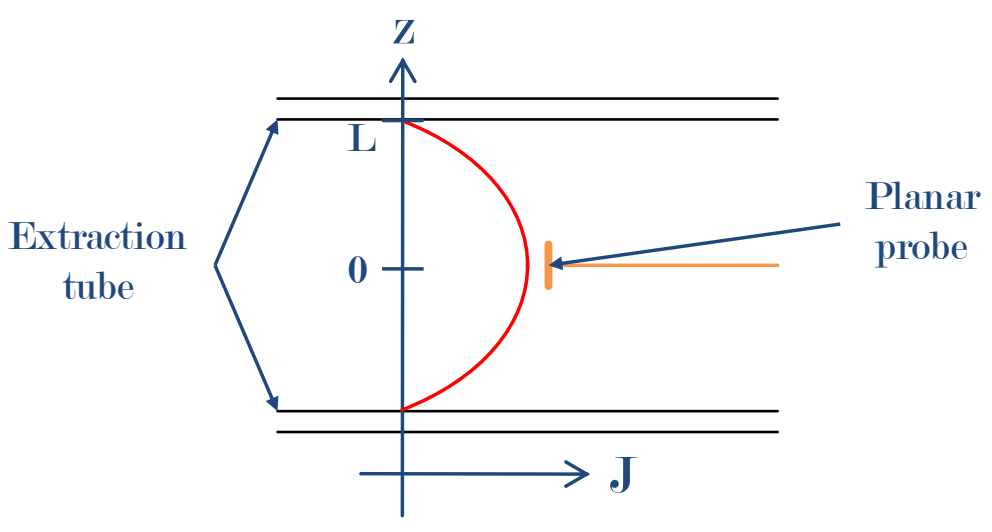

Figure 4.10: Cross-section of the assumed quadratic profile for the positive ion flux $\Gamma$ in the extraction tube. The planar probe is positioned at the center of the extraction tube.

The positive ion flux is calculated from positive ion current $I_{c}$ measurements done with a planar probe with an area $\mathcal{A}_{p}=28.3 \times 10^{-2} \mathrm{~cm}^{2}$. The positive ion flux is thus

$$
\Gamma_{+}=\frac{I_{c}}{e \mathcal{A}_{p}}
$$

As the thruster prototype cannot handle high powers in steady-state, positive ion flux measurements are done with pulsed power, between zero and the desired value. Moreover, the planar probe is not equipped with filtering inductances, which means that fast measurement cannot be done since an averaging is needed to obtain the direct-current component. A capacitor $C=100 \mathrm{nF}$ is thus placed in parallel of the measurement resistance $R=1 \mathrm{k} \Omega$, which results in a cut-off frequency of $f=50 \mathrm{kHz}$. During a cycle, the power is applied long enough to obtain the steady-state value of the current.

\subsubsection{Non-Uniformity of the Current Density}

The mass efficiency estimation makes an assumption that overestimates its value: the uniformity of the positive ion flux over the cross-section area of the extraction tube. This comes from the fact that the planar probe used for the measurement of the positive ion flux has an area $\mathcal{A}=28.3 \mathrm{~mm}^{2}$ much smaller than the cross-section area of the extraction tube, which is $17.6 \mathrm{~cm}^{2}$. The total positive ion flux is thus estimated by a measurement with a small area on axis. However, the positive ion flux is maximum on the axis of the extraction tube which corresponds to a radius of the thruster cylinder. The further away from the axis, the smaller the positive ion flux. The overestimation of the spatiallyaveraged positive ion flux means an overestimation of the mass efficiency.

In order to correct this, a profile approximation of the positive ion flux over the crosssection area is made. The positive ion flux is assumed to be zero at the wall of the extraction tube. A quadratic profile is assumed in each direction, with $z=0$ at the center of the extraction tube and $z= \pm L$ at the wall, as shown in figure 4.10. The positive ion flux value is $\Gamma_{\max }$ at $z=0$ (measurement with the planar probe), and zero at $z= \pm L$. 
Considering only one dimension, the positive ion flux can be written as

$$
\Gamma(z)=\Gamma_{\max }\left(1-\frac{z^{2}}{L^{2}}\right) .
$$

Integrating from $z=-L$ to $z=L$ yields

$$
\int_{-L}^{L} \Gamma(z) d z=\frac{4}{3} \Gamma_{\max } L
$$

The average in one dimension is thus

$$
<\Gamma>_{1 \mathrm{D}}=\frac{2}{3} \Gamma_{\max }
$$

As the cross-section of the extraction tube is rectangular, the total average is

$$
<\Gamma>_{2 \mathrm{D}}=\frac{4}{9} \Gamma_{\max }
$$

To take into account the profile of the positive ion flux, the mass efficiency is thus defined as

$$
M_{\text {eff }}=\left.\frac{4}{9} \frac{\Gamma_{+, \text {measured }}}{\Gamma_{\text {neutral }}}\right|_{\text {thruster exit }} .
$$

\subsubsection{Results}

Figure 4.11 shows the mass efficiency in percent as a function of power from $200 \mathrm{~W}$ to $1.4 \mathrm{~kW}$ peak power for argon and oxygen. The gas flow is set to $20 \mathrm{sccm}$ and the magnetic field to a coil current of $2 \mathrm{~A}$.

The first remarkable feature is the fact that the mass efficiency is relatively high at high power. Over $40 \%$ in argon and over $30 \%$ in oxygen at $P=1.4 \mathrm{~kW}$. Moreover, the evolution of the mass efficiency as a function of power shows a significant increase, proving that the design also yields high current densities for high powers.

It can also be seen that the mass efficiency for oxygen is smaller than for argon. Oxygen being a molecular gas $\left(\mathrm{O}_{2}\right.$ molecule), part of the energy coupled to the gas is used in molecule excitation and dissociation, instead of ionization and electron heating, as discussed in section 4.2.2. These higher energy losses per electron-ion pair created in molecular gases than in argon are a serious drawback for the PEGASES concept, which requires molecular gases by definition.

Although the mass is not taken into account for the mass efficiency, it should be noted that two types of positive ions can be found in oxygen: $\mathrm{O}_{2}^{+}$and $\mathrm{O}^{+}$. In the case of $\mathrm{O}^{+}$, it can be seen that half the mass of the neutral molecule $\mathrm{O}_{2}$ will be used for thrust, as opposed to $\mathrm{O}_{2}^{+}$ions. Therefore, the thrust efficiency is reduced when $\mathrm{O}^{+}$ions are not negligible compared to $\mathrm{O}_{2}^{+}$ions.

\subsection{Electron Temperature}

Measurements of the electron temperature were made very difficult by the radio-frequency noise due to capacitive coupling. The best conditions were obtained with the modified matchbox circuit and the three-coil antenna. The radial profile of the electron temperature, therefore, is only presented for this design. 


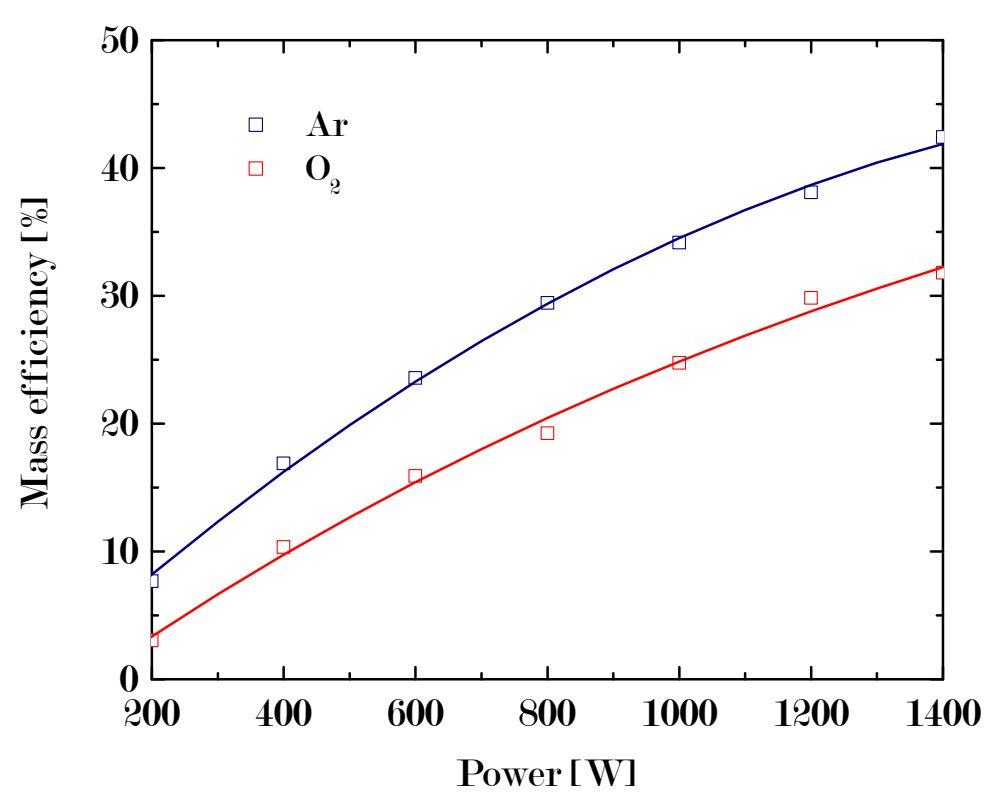

Figure 4.11: Mass efficiency in percent as a function applied power in $W$ for argon (blue curve) and oxygen (red curve). The gas flow is set at $20 \mathrm{sccm}$. The magnetic field is set to a coil current $I=2 \mathrm{~A}$. In each case, the curve is a polynomial fit of order 3 .

\subsubsection{Interest}

The electron temperature is an important parameter of the plasma as many processes depend on its value, as can be seen in table 6.1 (page 116) for reaction rates and collision frequencies in oxygen in chapter 6 where the magnetic electron filtering is modeled. It is, for instance, a good indication whether attachment to obtain negative ions is efficient.

Figure 4.12 shows the reaction rates in $\mathrm{m}^{3} \mathrm{~s}^{-1}$ for ionization and attachment in oxygen for an electron temperature from 1 to $10 \mathrm{~V}$. The two reaction rates can be compared as the source term is obtained by multiplying by the neutral and electron densities in both cases: $\nu_{\mathrm{iz}} n_{e}=K_{\mathrm{iz}} n_{g} n_{e}$ and $\nu_{\mathrm{att}} n_{e}=K_{\mathrm{att}} n_{g} n_{e}$. The two curves intersect at $T_{e}=2.2 \mathrm{~V}$. At this point, ionization and attachment are equal.

For high electron temperatures, the electrons are on average too energetic to attach on neutrals, even with dissociative attachment. This can be seen as the ionization reaction rate increases rapidly while the attachment reaction rate decreases slightly. For an electron temperature of $10 \mathrm{~V}$, the ratio between the ionization and attachment reaction rates is $3.2 \times 10^{2}$ (high ionization).

For low electron temperatures, the electrons are on average not energetic enough to ionize a neutral. The ionization reaction rate is seen to drop rapidly as the electron temperature is decreased. The attachment rate also drops as the electron temperature is decreased because it is a dissociative attachment, which means that a minimum energy is required to dissociate the oxygen molecule. With an electron temperature of $1 \mathrm{~V}$, the ratio of the attachment to ionization reaction rates is $1.9 \times 10^{2}$ (high attachment).

The profiles of these reaction rates, however, depend strongly on the type of feedstock gas. For instance in sulfur hexafluoride, the attachment is not dissociative, which means that there is no energy threshold for electrons. As a result, the attachment reaction rate is a rapidly increasing function of decreasing electron temperature. 


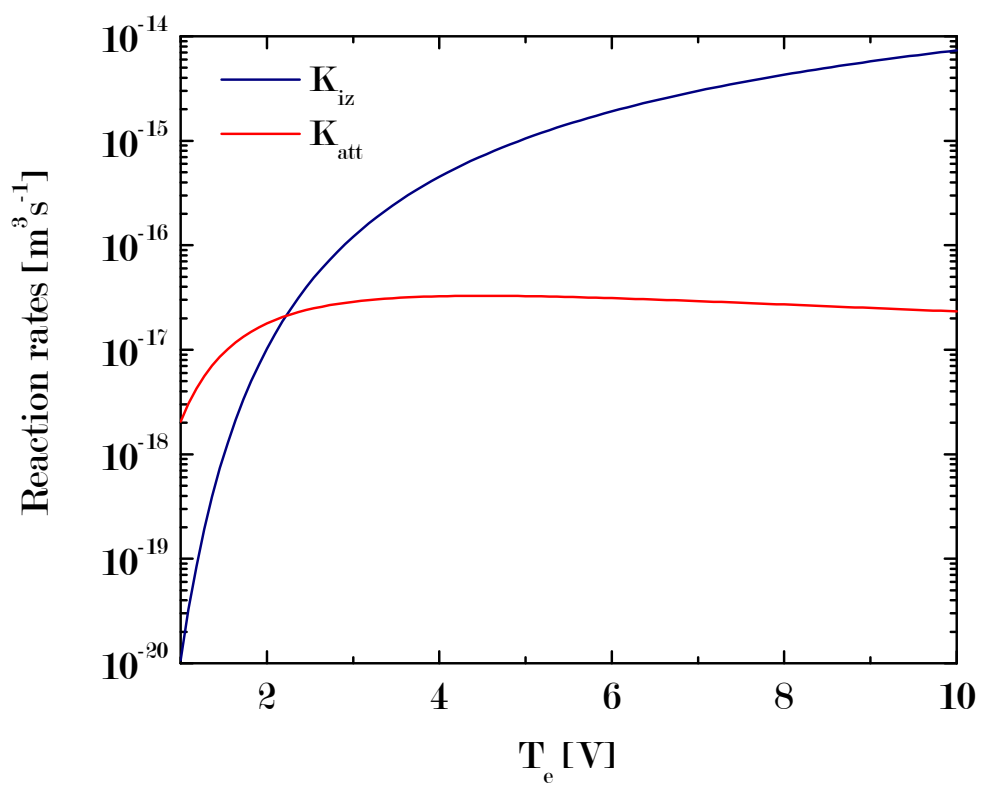

Figure 4.12: Semi-logarithmic plot of the reaction rates in $m^{3} s^{-1}$ for ionization (blue curve) and attachment (red curve) in oxygen.

\subsubsection{Discussion of the Results}

The electron temperature profile on the radius of the thruster through the left hand side extraction tube, as shown in figure 4.13, was investigated for argon (figure 4.13a) and oxygen (figure 4.13b). Two gas flows were chosen: one corresponding to the maximum of current density for argon (20 sccm, squares) and one for oxygen (50 sccm, circles). Two applied powers were also chosen: one corresponding to the studies for the current density (300 W, blue) and one at higher power $(600 \mathrm{~W}$, red). The magnetic field was set to a coil current of $1.5 \mathrm{~A}$, which seemed to correspond to maximum current density values for both gases. The interface between the thruster cylinder and the extraction tubes is at $r=2.9 \mathrm{~cm}$, and the extraction tubes edge is at $r=8.6 \mathrm{~cm}$. The high electron temperature values in the center come from the fact that this is a very low pressure case, as discussed below.

On top of the filtering inductances, averaging methods were used to reduce the noise in the measurements. During a voltage scan to obtain the I-V characteristic, the value for each voltage point was the average of 20 measurements. Each voltage scan for each position was done three times. These averagings were done with the Scientific System box, described in section 2.2.5. All these steps were done twice for each position, which means that the final $\mathrm{I}-\mathrm{V}$ characteristic is the average of the two averaged characteristics. The analysis of the characteristics was done with lab-made programs, where the parameters of the analysis could be varied (see section 2.2.5) for improved understanding.

\section{Magnetic Field Effect}

The first aspect of the electron temperature profiles, for all conditions, is the gradient between the center of the thruster cylinder and the extraction tube. A high electron temperature is found at the center of the thruster cylinder, while the extraction tube is characterized by a low electron temperature down to $2 \mathrm{~V}$. The electron temperature gradient is localized in the thruster cylinder as there is little evolution between $r=2.9 \mathrm{~cm}$ 


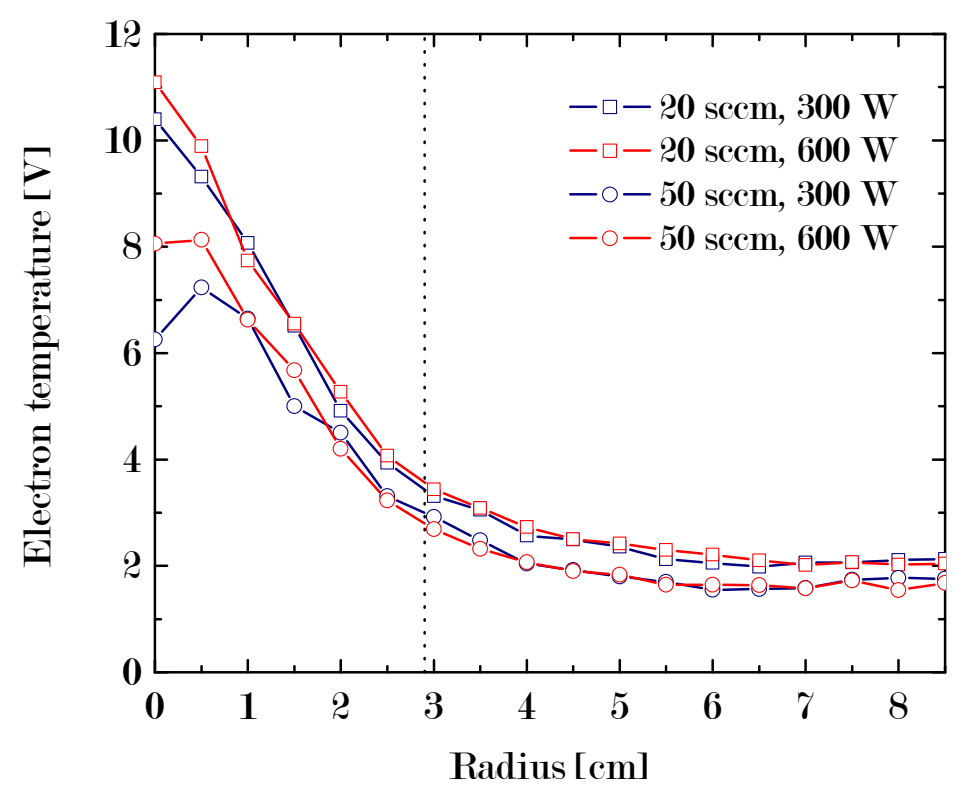

(a) $\mathrm{Ar}$

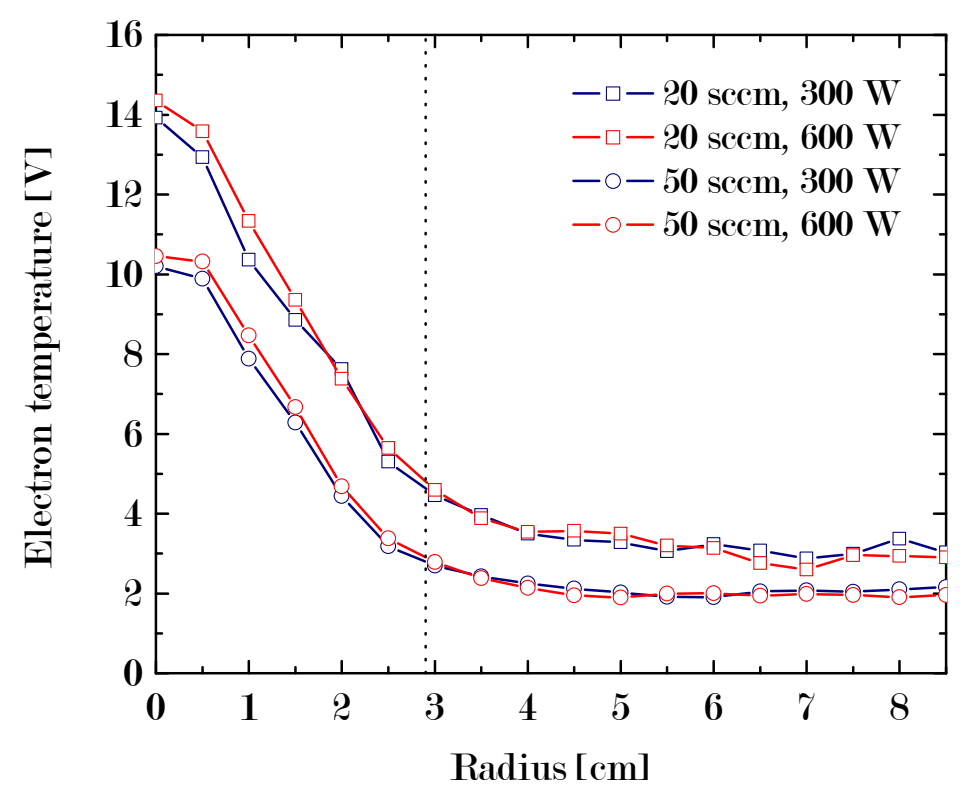

(b) $\mathrm{O}_{2}$

Figure 4.13: Electron temperature in $V$ as a function of radius in $\mathrm{cm}$ through the left hand side extraction tube in argon (a) and oxygen (b) for two gas flows, $20 \mathrm{sccm}$ (squares) and $50 \mathrm{sccm}$ (circles), and two applied powers, $300 \mathrm{~W}$ (blue) and $600 \mathrm{~W}$ (red). The magnetic field was set to a coil current of $1.5 \mathrm{~A}$. In both figures, the dotted line represents the edge between the thruster cylinder and the extraction tube. 
and $r=8.5 \mathrm{~cm}$. This localization of the high temperature for electrons is due to the way they are heated in the discharge. The antenna wrapped around the middle of the thruster cylinder heats the electrons in two ways: in the volume directly surrounded by the antenna via capacitive and inductive modes, and along the magnetic lines crossing this volume via a helicon mode. As the magnetic field lines are roughly parallel to the thruster cylinder, this means that there is no heating in the extraction tube $(r>2.9 \mathrm{~cm})$. Since the crossfield diffusion of electrons occurs through collisions, electrons lose energy as they travel in the radial direction, which explains the gradient of electron temperature between the center of the thruster cylinder and the extraction tubes. Once in the extraction tube, the magnetic field becomes much weaker and no longer confines the electrons. As they can diffuse freely, there is no gradient of electron temperature in the extraction tube.

Therefore, the plasma is divided into two regions: the thruster cylinder with high electron temperatures, and the extraction tube with a low electron temperature. As a result, the inelastic processes are very different between these two regions (figure 4.12). In the thruster cylinder, the high electron temperatures lead to an efficient generation of positive ions since the dominating source term is the ionization term. As the attachment term is very small compared to the ionization term, very few negative ions are produced. With a very small fraction of negative ions, the electronegative plasma is very similar to an electropositive plasma. As the plasma diffuses radially (across the magnetic field), the electron temperature drops to a low value around $2 \mathrm{~V}$ as it enters the extraction tube. Here, the attachment term is greater than the ionization term, leading to a more efficient creation of negative ions as electrons are lost. The effect of the magnetic field is thus double. First, as the plasma diffuses across the magnetic field lines, electrons are confined and the negative ion fraction increases, which results in an ion-ion plasma as electrons are depleted. Second, the magnetic field separates the plasma into a region where the creation of positive ions and electrons is efficient (ionization), and another one where the creation of negative ions and loss of electrons are efficient.

\section{Gas, Pressure and Power Effects}

To discuss these effects in a very simplified way, the particle balance of an electronegative plasma needs to be considered. Considering a global model, an approximation of the particle balance can be written as

$$
\left(K_{\mathrm{iz}}-K_{\mathrm{att}}\right) n_{e} n_{g} \mathcal{V}=h_{\mathrm{EN}}\left(n_{e}+n_{-}\right) u_{B} \mathcal{A}-K_{\mathrm{rec}} n_{+} n_{-} \mathcal{V},
$$

where $\mathcal{V}$ is the volume of the plasma, $\mathcal{A}$ the area of the plasma walls, and $h_{\mathrm{EN}}$ a factor representing the plasma density drop between the plasma center and the sheath edge in an electronegative plasma. It should be noted that the magnetic field is not explicitly considered, but would play a role in this factor. This particle balance is composed of the electron creation (first term), positive ion loss at the walls (second term), and volume ion recombination loss (third term). In order to further simplify the particle balance, the recombination loss is assumed negligible compared to the wall loss, yielding

$$
K_{\mathrm{iz}}-K_{\mathrm{att}}=\frac{h_{\mathrm{EN}}(1+\alpha) u_{B}}{n_{g}} \frac{\mathcal{A}}{\mathcal{V}} .
$$

It should be noted that in the case of an electropositive plasma $(\alpha=0)$, this relation becomes

$$
K_{\mathrm{iz}}=\frac{h_{\mathrm{EP}} u_{B}}{n_{g}} \frac{\mathcal{A}}{\mathcal{V}},
$$


where $h_{\mathrm{EP}}$ is the edge-to center density ratio in the electropositive case, different from $h_{\mathrm{EN}}$.

Gas Effect The radial electron temperature profiles in argon and oxygen are very similar, except for the value of the electron temperature in the thruster cylinder: it is higher in oxygen. This difference is due to the fact that argon is electropositive while oxygen is electronegative. As can be seen in (4.13), an increase in electronegativity $\alpha$ results in an increase of $\left(K_{\mathrm{iz}}-K_{\mathrm{att}}\right)$, which is an increasing function of the electron temperature. As a result, the electron temperature in an electronegative plasma is often higher than that in an electropositive plasma for similar plasma conditions.

Pressure Effect Two values of gas flow, hence pressure, were investigated: 20 and $50 \mathrm{sccm}$. As the gas flow is increased, whether in argon or oxygen, the electron temperature is seen to drop by $1 \mathrm{~V}$ in the extraction tube, and a few volts in the thruster cylinder. Assuming that the neutral temperature is constant, the pressure is directly proportional to the neutral density $\left(n_{g}\right)$. Moreover, $h_{\mathrm{EN}}$ and $h_{\mathrm{EP}}$ are decreasing functions of the pressure. It follows from (4.13) and (4.14) that the electron temperature is a decreasing function of pressure.

Power Effect Whether in argon or oxygen, the electron temperature is relatively independent of the applied power. This is confirmed by the particle balance in (4.13) and (4.14) where the applied power (or electron density $n_{e}$ ) does not appear. A slight increase in electron temperature, however, can be seen for all conditions (gas and pressure). This can be explained by assuming that neutral depletion occurs in the plasma, and results in a decrease in neutral density. This decrease in neutral density is more pronounced at higher powers. An increase in applied power, therefore, results in an increase of electron temperature $[54,55,56,57,58]$. It should be noted that the effect of neutral depletion on the particle balance is the subject of Laurent Liard's thesis in our laboratory.

\subsection{Conclusion}

The ionization stage of the PEGASES thruster prototype I was investigated. Changes first needed to be made to improve the power coupling. Different plasma parameters were studied to understand the plasma equilibrium as a function of plasma conditions.

\subsubsection{Power Coupling}

Our design of the Boswell-type (double-saddle) antenna failed to produce high density plasmas. The helicon mode that was aimed at, could not be reached, and short-circuits ruined the power coupling.

The simpler three-coil design, capable of exciting the $m=0$ and $m= \pm 1$ modes, showed a satisfactory power coupling. Helicon waves could be launched with this antenna, and high density plasmas were produced.

In an effort to obtain a symmetrical voltage distribution over the antenna, the matchbox circuit was modified (switch between the tune capacitor and the antenna). The resulting voltage distribution was similar to the one before modification, leaving the plasma roughly unchanged. 
The study of plasma asymmetry and instabilities showed the limitations of the double helicon design. For a thruster, symmetry is required to avoid any rotational effect. As the PEGASES thruster prototype I consistently showed asymmetrical positive ions current densities, the design of two extraction tubes should be changed to one extraction zone. Moreover, the plasma diffusion, first in the thruster cylinder and then in the extraction tube, is a cause of instability in the plasma equilibrium. The design of the PEGASES thruster prototype II, presented in section 8.4.2, takes these observations into account.

\subsubsection{Positive Ion Current Density}

With the positive ion current density measurements in the extraction tube, whether close to the thruster cylinder or to the outer edge of the extraction tube, the influence of the plasma conditions was investigated. The two main parameters considered were the magnetic field and the gas flow. For each parameter, the effect on the positive ion current density is twofold. This results in intermediate maxima in the range of parameters considered. As a function of magnetic field, the maxima were found around a coil current $I= \pm 2 \mathrm{~A}$, in a range from -3 to $3 \mathrm{~A}$. For the gas flow, the maximum was found around $20-50 \mathrm{sccm}$, in a range from 5 to $80 \mathrm{sccm}$.

The influence of the gas composition and the applied power were also studied. Both gases, argon and oxygen, were found to behave similarly, with lower current densities for oxygen. As to the applied power, it does not change the plasma equilibrium nature, but increases the positive ion current density as it is increased.

\subsubsection{Mass Efficiency}

When it comes to thrusters, the mass efficiency is an important parameter since the available power is limited. It consists of comparing the positive ion flux to the neutral flux. Its value at high power $(P=1.4 \mathrm{~kW})$ is good (over $40 \%$ in argon).

However, one drawback was expected, and unfortunately, confirmed: the mass efficiency is significantly lower for electronegative gases. At $P=1.4 \mathrm{~kW}$, the mass efficiency in argon and oxygen is around $40 \%$ and 30\% respectively. This is due to the molecular nature of electronegative gases: the collisional energy lost per electron-ion pair created is significantly higher compared to noble gases.

\subsubsection{Electron Temperature}

The radial profile of the electron temperature was investigated from the center of the thruster cylinder, i.e. the core of the ionization stage, to the outer edge of the extraction tube. What was found is a high temperature gradient between the two regions. Due to the low pressure of the plasmas considered, the electron temperature is high where the electron heating occurs (around $10 \mathrm{~V}$ ). Once in the extraction tube, the electrons are no longer heated. Since the magnetized electrons diffuse cross-field through collisions, their temperature drops rapidly. The dependency of the electron temperature on the plasma conditions was explained using simple particle balance considerations.

It follows from the electron temperature profile that the plasma is divided into two regions. In the core of the ionization stage, the creation of electrons and positive ions is efficient due to the high electron temperature. In the extraction region, the attachment 
of cooled electrons on neutrals is efficient (low electron temperature). This observation will be used to improve the PEGASES thruster concept. 


\section{Chapter 5}

\section{Magnetic Electron Filtering Stage - Experiments}

\section{Contents}

5.1 Experimental Background on Ion-Ion Plasmas . . . . . . . 82

5.1 .1 Ion-Ion Plasma Creation . . . . . . . . . . . . . . . 82

5.1 .2 Electronegative Plasma Afterglow . . . . . . . . . . . . . . . . . . . . . 82

5.1 .3 Magnetic Electron Filtering . . . . . . . . . . . . . . . . . . . . . . . . . . 83

5.2 Helicon Reactor ................ 83

5.2 .1 Electron Density and Positive Ion Flux . . . . . . . . . . 83

5.2 .2 I-V Characteristics . . . . . . . . . . . . . . . . . 86

5.2.3 Plasma and Floating Potentials, Electron Temperature . . . . . 89

5.2 .4 RFEA Measurements. . . . . . . . . . . . . . . . 92

5.2 .5 Electronegativity Measurements . . . . . . . . . . . . 95

5.3 PEGASES Prototype I . . . . . . . . . . . 96

5.3 .1 I-V Characteristics . . . . . . . . . . . . . . . . . . 97

5.3 .2 Electronegativity Measurements . . . . . . . . . . . . . . . . . . 97

5.4 Design Improvements . . . . . . . . . . . . . . 100

5.4 .1 Enhanced Magnetic Filtering . . . . . . . . . . . . . . 100

5.4 .2 Optimized Neutral Injection . . . . . . . . . . . . . . 102

5.5 Conclusions .................... 103

The ion-ion plasma needed for the third stage (ion extraction and acceleration) of the PEGASES thruster can be obtained using two methods: temporal (plasma afterglow) and spatial filtering (magnetic field). The resulting ion-ion plasmas have different properties. For the PEGASES thruster, the choice of a static magnetic field is made. It confines the electrons to the center of the discharge, leaving the positive and negative ions free to diffuse outward together with the quasi-neutrality condition: in the absence of the electrons and assuming that the ions have the same charge, the positive and negative ion densities should be equal.

Since the first ion-ion plasmas were obtained in the helicon reactor and the first PEGASES thruster prototype was not ready until fall 2007, the formation and structure of 
ion-ion plasmas were first investigated in the helicon reactor. Among the plasma parameters considered was the electronegativity (ratio of negative to electron density), which is an essential parameter to understand the formation of an ion-ion plasma.

Once the thruster chamber and prototype were functional, ion-ion plasmas were investigated in the thruster itself. The obtention of an ion-ion plasma in the extraction tubes, however, proved impossible to achieve. The electronegativity was measured to understand the mechanisms for the formation of negative ions.

Using the results from the ion-ion plasma formation studies, design improvements have been tested on the first PEGASES thruster prototype. They consist of enhancing the magnetic electron filtering with additional magnets, and optimizing the neutral injection.

\subsection{Experimental Background on Ion-Ion Plasmas}

\subsubsection{Ion-Ion Plasma Creation}

Two methods can be used to obtain an ion-ion plasma: the afterglow of an electronegative plasma (temporal filtering), or a stratified plasma using a constant magnetic field (spatial filtering). In the first case, the ion-ion plasma only exists for a short amount of time: when the power is switched off, the cooling electrons are quickly attaching to neutrals, but the recombinations, whether electron-ion or ion-ion, destroy the plasma. In the second case, the constant magnetic field is used to confine the electrons to an electronegative region (core if the symmetry is cylindrical) and this results in an electron-free region (periphery of the core).

\subsubsection{Electronegative Plasma Afterglow}

Ion-ion plasmas are defined as plasmas in which the electron density and temperature are low enough that the current carried by negative charges is dominated by the negative ions. In the case of afterglow plasmas, the plasma is first electron dominated (power on), then negative ion dominated (power off) [59]. The afterglow can be divided into two stages $[60,61]$. In the first stage, the negative ions are still trapped in the electronegative region, as opposed to the electropositive region close to the walls, while electrons are lost through recombination at the walls or attachment to neutrals. In the second stage, electrons are negligible and both positive and negative ions are lost through recombination while diffusing ambipolarly. Ion-ion plasmas are possible in afterglow plasmas because of the different timescales for the two stages: the electron diffusion is a lot faster than the ion diffusion. However, if the detachment frequency is too high, negative ions are converted into electrons, which prevents the creation of an ion-ion plasma.

Time resolved measurements with a Langmuir probe show the transition between an electron dominated plasma and a negative ion dominated plasma [62]: from an asymmetrical I-V characteristic to a symmetrical I-V characteristic. Comparison between an electropositive gas and an electronegative gas, for instance argon and oxygen [63], show that the electron density decay is faster in an electronegative plasma due to attachment. Finally, the duty cycle and the pulse repetition frequency play an important role in the afterglow plasma [64]. 


\subsubsection{Magnetic Electron Filtering}

By definition, an afterglow plasma cannot produce a steady-state ion-ion plasma. However, it is possible to do so with the method of magnetic electron filtering. The idea is to use a constant magnetic field to confine the electrons in one region, while the ions, both positive and negative, diffuse to a second region. Due to the mass ratio between electrons and ions, there is a range of magnetic field value for which only the electrons are magnetized. The magnetic filtering of electrons can only be achieved in electronegative gases, where negative ions are produced. Without those negative ions, the positive ion diffusion would be impeded because of quasi-neutrality: as soon as ions diffuse outside of the electron confinement region, electric forces pull them back toward the confinement region.

Different geometries can be used. The electronegative region can be a sheet [65] generated by an electron beam, itself created by an emitting cathode. In this case, the ion extraction, whether positive or negative, can be done on either side of the sheet. The electronegative and ion-ion regions can also be separated by a magnetic filter [66], consisting of an array of magnets. The plasma chamber is thus cut into the two regions. Finally, the geometry can be cylindrical [67, 44], with an electronegative core and an ion-ion periphery (the filtering occurs along the radius of the discharge).

\subsection{Helicon Reactor}

This section focuses on results obtained in the helicon reactor.

The electron density and positive ion flux profiles, I-V characteristics, the plasma and floating potential profiles, and measurements with a retarding field energy analyzer were considered. Finally, the electronegativity is measured as a function of radius to understand the creation of negative ions in the cross-field diffusion.

\subsubsection{Electron Density and Positive Ion Flux}

The idea of the magnetic electron filtering consists of separating the electrons from part of the positive ions and the negative ions. In order to investigate this separation, the electron density profile is compared to the positive ion flux profile over the radius of the diffusion chamber for three gases: argon (electropositive gas), oxygen (weakly electronegative gas), and sulfur hexafluoride (electronegative gas). The electron density is obtained by measuring the current collected by a filament probe biased to the plasma potential. The current collected by a planar probe biased to $-60 \mathrm{~V}$ is used to measure the positive ion flux.

Figure 5.1 shows the radial profiles of the electron density (figure 5.1a) and the positive ion flux (Figure 5.1b), normalized to their value at the center. The same pressure of $p=0.5$ mTorr was used for all gases. For the measurements of the electron density with a filament probe, the power was set to $P=500 \mathrm{~W}$ and the magnetic field to $B=120 \mathrm{G}$ with a coil current $I=8.5 \mathrm{~A}$. For the positive ion flux measurements with a planar probe, the power was set to $P=700 \mathrm{~W}$ and the magnetic field to $B=140 \mathrm{G}$ with a coil current $I=10 \mathrm{~A}$. The difference in power and magnetic field between the two types of measurements does not prevent the comparison between them and is due to an attempt at increasing the positive ion flux. The comparison of the radius where the profiles drop, however, is not possible. It should be noted that the radius of the source 


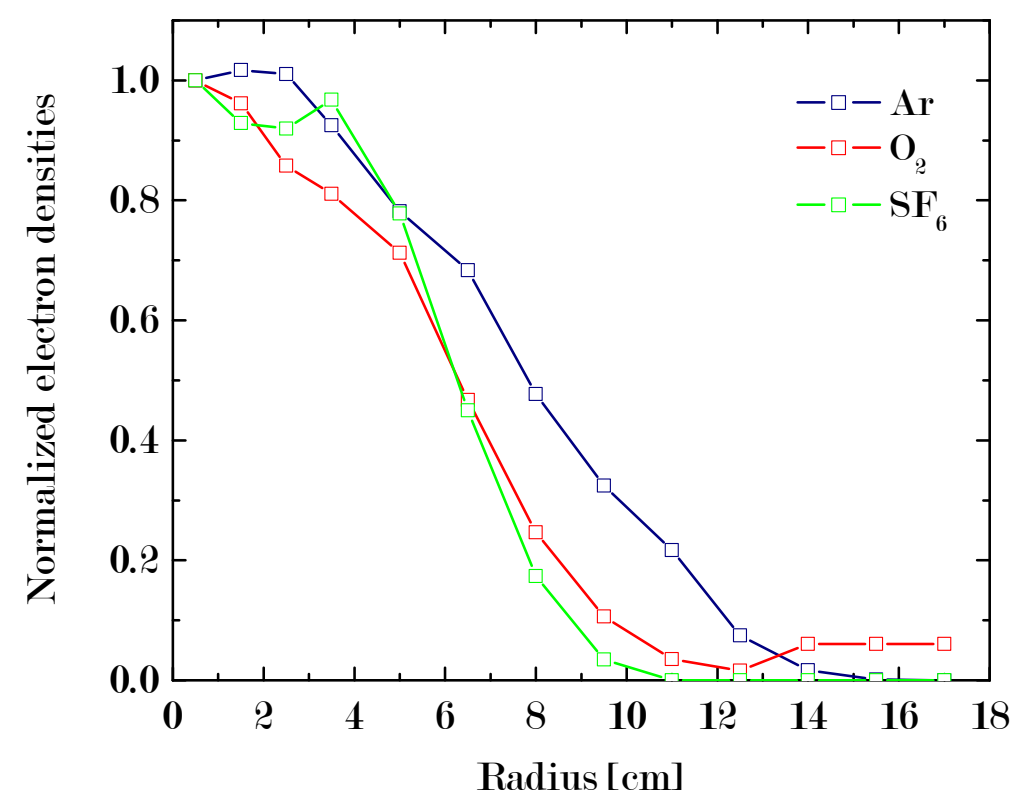

(a)

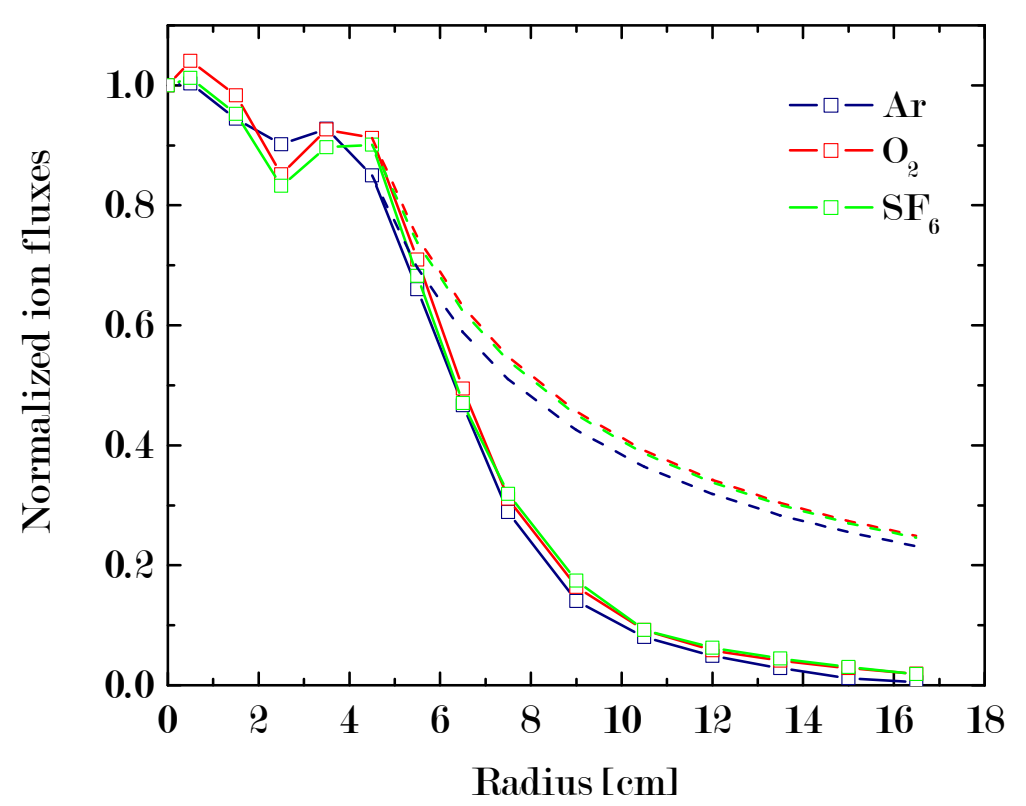

(b)

Figure 5.1: Electron density (a) and positive ion flux (b) profiles as functions of the radius of the diffusion chamber for argon (blue), oxygen (red) and sulfur hexafluoride (green) at $p=0.5$ mTorr. Electron density measurements were done at $P=500 \mathrm{~W}$ and $B=120 G$. Positive ion flux measurements at $P=700 \mathrm{~W}$ and $B=140 G$. The dashed lines in figure (b) correspond to a cylindrical diffusion from $r_{a}=4.5 \mathrm{~cm}$. 
region is $r=6.5 \mathrm{~cm}$ (see figure 3.1 in section 3.1). The electron heating, therefore, occurs between $r=0 \mathrm{~cm}$ and around $r=6.5 \mathrm{~cm}$. Beyond this radius, both profiles show a strong decrease because of the cylindrical geometry and the lack of electron heating. The local maximum around $r=4 \mathrm{~cm}$ in the positive ion flux profile may be due to the inductive heating near the antenna in the source region, which transfers in the diffusion chamber along the magnetic field lines. It cannot be seen in the electron density profiles, except for sulfur hexafluoride, probably because of the difference in applied power.

The electron density at the center, to which the normalization is done, is $4.1 \times 10^{17} \mathrm{~m}^{-3}$ in argon, $1.2 \times 10^{17} \mathrm{~m}^{-3}$ in oxygen, and $1.7 \times 10^{17} \mathrm{~m}^{-3}$ in sulfur hexafluoride. The role of the gas can clearly be seen on the electron density profile. In an electropositive gas, the electrons and positive ions cannot be separated because of quasi-neutrality. The diffusion, same for electrons and positive ions, is called ambipolar diffusion. In the case of an electronegative plasma, quasi-neutrality can be fulfilled with the two ion species. Since the electron diffusion does not affect the ion diffusion, there can be a separation of the electrons from the ions. In argon, the electrons are de-confined by the outgoing positive ions, corresponding to an ambipolar diffusion with reversed roles for electrons and positive ions. In the absence of a static magnetic field, the ambipolar diffusion results from the electrons slowed down by the slower ion diffusion. Oxygen is a weakly electronegative gas. As a consequence, a small separation between electrons and ions can be seen. This separation, however, is more pronounced in sulfur hexafluoride, which is a much more electronegative gas.

Contrary to the electron density profile, the positive ion flux profile does not change with the gas, whether electropositive or electronegative. In the case of an infinite cylinder, and assuming that there are no volume losses, the conservation of the flux across the radius can be written as

$$
\nabla\left(\Gamma_{+}\right)=\frac{1}{r} \frac{d\left(r \Gamma_{+}\right)}{d r}=0
$$

By choosing an arbitrary point as the start of the decrease, for instance $r_{a}=4.5 \mathrm{~cm}$, the positive ion flux as a function of radius can be written as

$$
\Gamma_{+}(r)=\Gamma_{+}\left(r_{a}\right) \frac{r_{a}}{r}
$$

and is shown as dashed lines on figure 5.1. The positive ion flux for all gases clearly does not follow this scaling. It was seen in section 1.2.2 that the positive ion-negative ion recombination is faster than the electron-positive ion recombination. If the only effect was the volume recombination, the positive ion flux profile in argon would be higher than that of the electronegative gases. Taking into account the axial losses along the magnetic field lines provides a possible explanation. As the electrons dominate the plasma equilibrium in the axial direction (no magnetic confinement), electrons and positive ions are lost at the chamber ends. This additional axial loss will be used for the fluid model on the magnetic electron filtering in chapter 6 . The fact that the decrease of the positive ion flux is stronger than the $r^{-1}$ scaling comes from these axial losses. In argon, electrons and positive ions are lost axially at the chamber ends and by electron-positive ion recombination in the volume. In oxygen and sulfur hexafluoride, the axial losses should be smaller as electrons are attached and produce negative ions, but the volume losses (positive ion-negative ion recombination) are greater (see section 1.2.2). Although the loss mechanisms are different between the electropositive gas (argon) and the electronegative gases (oxygen and sulfur hexafluoride), the positive ion flux profile is similar for all three gases. 
As a conclusion, the magnetic filtering of electrons can only occur in an electronegative gas, where the negative ions allow the fulfillment of quasi-neutrality without electrons. As the negative ion fraction increases, stronger electronegativity, the separation between electrons and ions increases.

\subsubsection{I-V Characteristics}

In an electropositive plasma, an I-V characteristic is composed of three regions. Above the plasma potential, only the collected current is an electron current which increases as the bias is increased because of sheath effects. Below the plasma potential, positive ions, as well as fast electrons, are collected. As the fraction of electrons capable of overcoming the potential barrier decreases exponentially (Boltzmann assumption), the collected current decreases exponentially until no electrons are collected. The floating potential can be found in this region. The third region corresponds to only positive ions being collected, with an increase in current as the bias decreases to negative values due to sheath effects. Because of the mass ratio between electron and positive ions, the electron current is greater than the positive ion current. The shape of the I-V characteristic in such a case was given in figure 2.1 in section 2.2.1.

In an electron-free plasma, i.e. ion-ion plasma, the only negatively charged species is the negative ions. With a mass and a temperature comparable to that of positive ions, the negative ion current is similar to the positive ion current. The I-V characteristic in an ion-ion plasma, therefore, is symmetrical around the plasma potential.

I-V characteristics in electronegative plasmas depend on the negative ion fraction. For a small negative ion fraction, the electron current is dominating the negative species current, and I-V characteristics are similar to electropositive ones. With increasing negative ion fraction, the negative species current decreases as it is a combination of the electron current (high) and the negative ion current (small). When the electrons become negligible, the I-V characteristic becomes similar to that of an ion-ion plasma.

\section{Radial Profiles}

The evolution of I-V characteristics, measured with a filament probe in the diffusion chamber of the helicon reactor, as a function of radial position is showed in figure 5.2. The pressure was set to $p=0.5$ mTorr with the plasma turned on, for an applied power of $P=500 \mathrm{~W}$ and a magnetic field of $B=120 \mathrm{G}$ for a coil current of $I=8.5 \mathrm{~A}$. Each $\mathrm{I}-\mathrm{V}$ characteristic is normalized to its maximum absolute value.

In the case of argon (figure 5.2a), the I-V characteristic is asymmetrical over the whole radius. This was to be expected as there are no negative ions in an electropositive plasma. The noise seen in the electron current for $R=15 \mathrm{~cm}$ is due to the very low plasma density at this point, as it was seen above (section 5.2.1).

In oxygen (figure 5.2b), electrons dominate the negative current over most of the radius. At $r=15 \mathrm{~cm}$ however, the $\mathrm{I}-\mathrm{V}$ characteristic is symmetrical, which indicates that electrons are negligible. The plasma beyond this radius can be considered an ion-ion plasma as the currents on each side of the plasma potential are equal.

Finally for sulfur hexafluoride (figure 5.2c), the electrons become negligible at a smaller radius, since it can be seen that the $\mathrm{I}-\mathrm{V}$ characteristic is becoming symmetrical at $r=$ $10 \mathrm{~cm}$. Further away from the center, the negative ions dominate the plasma equilibrium. 


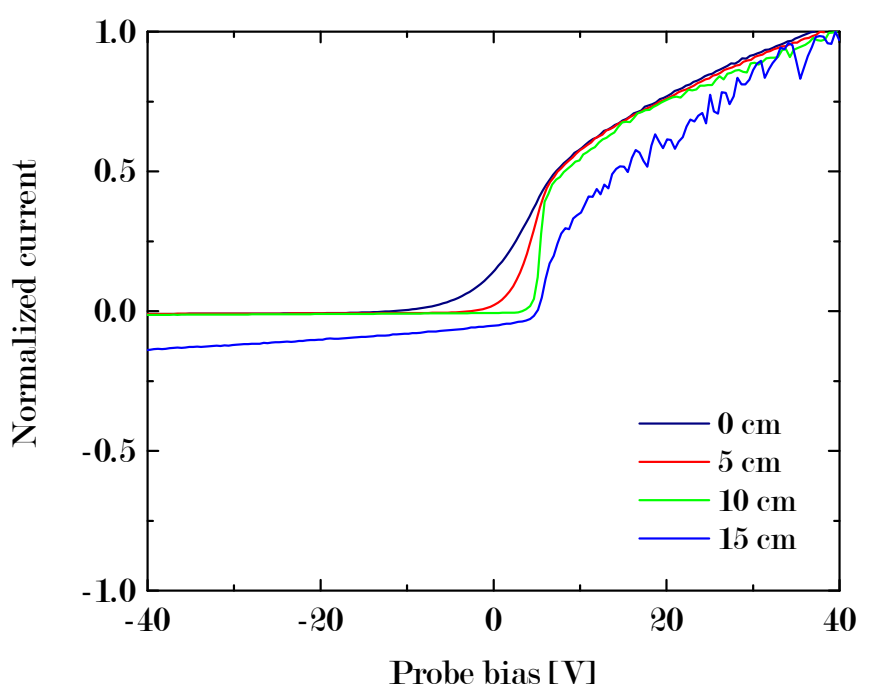

(a) $\mathrm{Ar}$

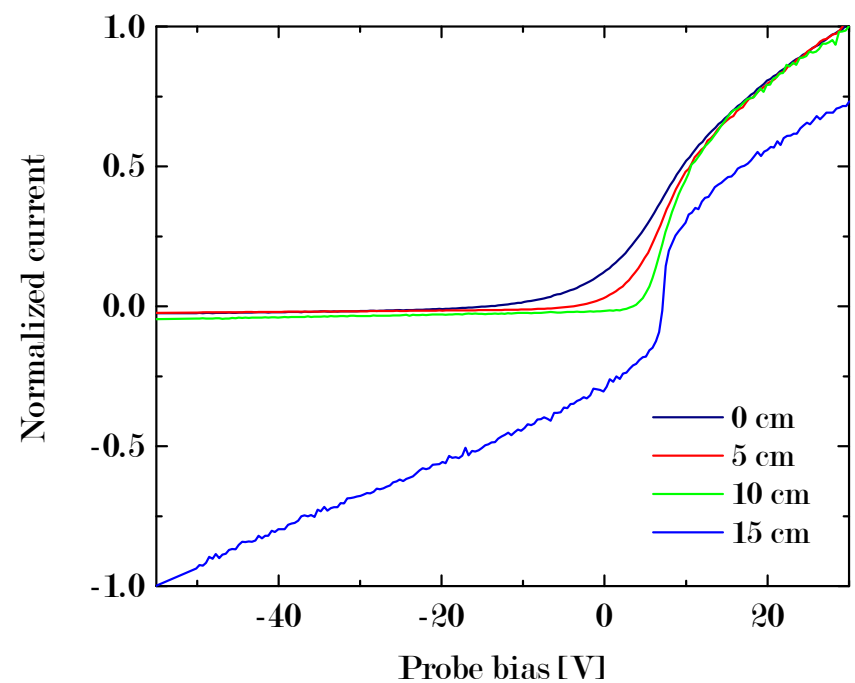

(b) $\mathrm{O}_{2}$

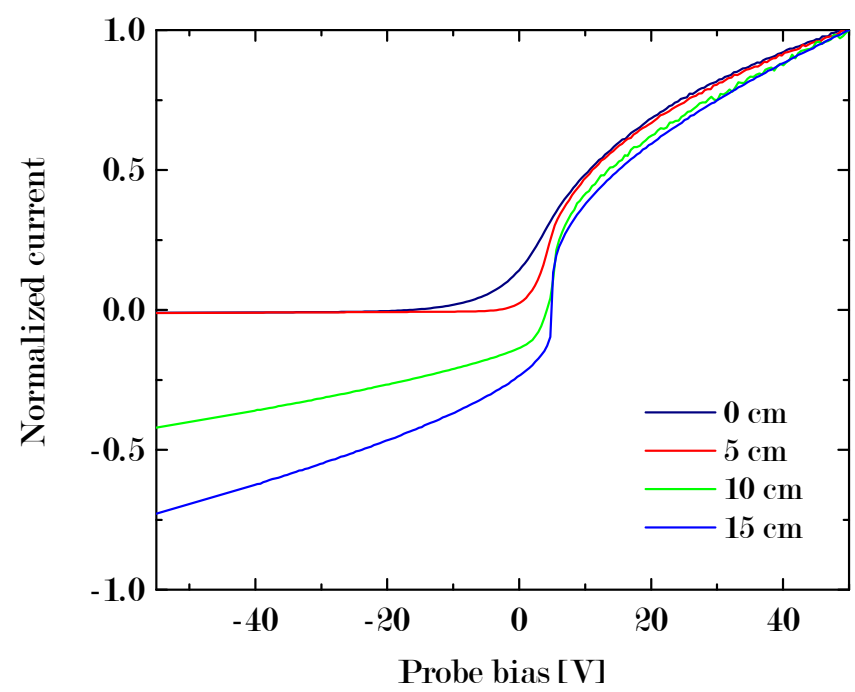

(c) $S F_{6}$

Figure 5.2: $I$ - $V$ characteristic as a function of radial position $(r \in\{0,5,10,15\} \mathrm{cm})$ in argon (a), oxygen (b) and sulfur hexafluoride (c). Each characteristic is normalized to its maximum absolute value. The gas pressure is $p=0.5 \mathrm{mTorr}$ when the plasma is on, applied power of $P=500 \mathrm{~W}$, and magnetic field of $B=120 \mathrm{G}$ for a coil current of $I=8.5 \mathrm{~A}$. 


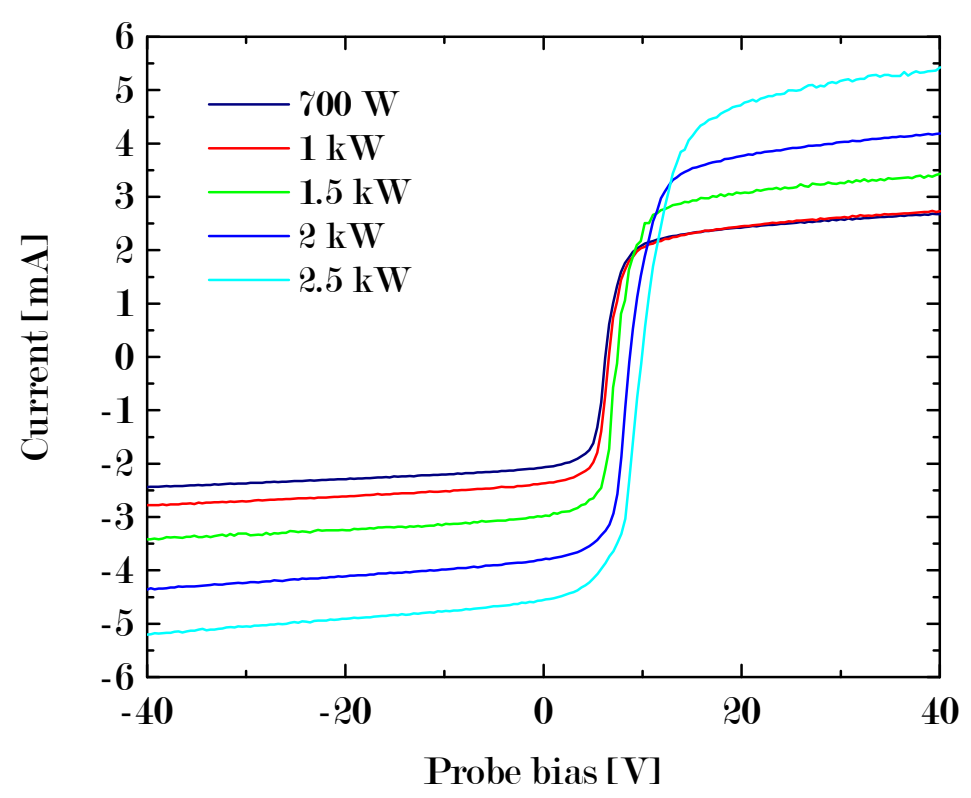

Figure 5.3: $I-V$ characteristics in sulfur hexafluoride as a function of applied power with $P \in\{0.7,1,1.5,2,2.5\} \mathrm{kW}$. Measurements were done with a planar probe set at $r=14 \mathrm{~cm}$ for a pressure $p=0.5$ mTorr with plasma on and $B=140 \mathrm{G}$ for a coil current $I=10 \mathrm{~A}$.

As it was described in section 2.2.1, the exponential decay of the electron current as the probe bias is decreased from the plasma potential is a function of the electron temperature. Calculations of the electron temperature as a function of radius were shown in figure 4.13 in section 4.4. The evolution of the electron temperature, however, can be described qualitatively with I-V characteristics: the potential difference $V_{p}-V_{f}$ is an increasing function of the electron temperature. What is seen for all gases is an electron temperature decrease as the radius increases. In the case of an electronegative gas, this decrease occurs until the symmetrical profile of an ion-ion plasma is reached. Negative ions have a lower temperature than electrons due to their greater mass. As they become dominant over electrons, the decrease in potential difference $V_{p}-V_{f}$ confirms the transition towards an ion-ion plasma.

\section{Power Effect}

Figure 5.3 shows I-V characteristics as a function of power from $700 \mathrm{~W}$ to $2.5 \mathrm{~kW}$ at $r=14 \mathrm{~cm}$, obtained in sulfur hexafluoride with a planar probe. The gas pressure was set at $p=0.5 \mathrm{mTorr}$ when the plasma was on. The magnetic field to $B=140 \mathrm{G}$ with a coil current of $I=10$ A.

It can be seen that at the radial position $r=14 \mathrm{~cm}$, the plasma behaves as an ionion plasma for all applied powers considered, with the equal ion currents an increasing function of power. Moreover, it can be seen that the plasma potential, equal to the floating potential in ion-ion plasmas, is also an increasing function of power, going from $5 \mathrm{~V}$ at $700 \mathrm{~W}$ to $10 \mathrm{~V}$ at $2.5 \mathrm{~kW}$. The plasma and floating potentials are discussed below. 


\subsubsection{Plasma and Floating Potentials, Electron Temperature}

In an ideal ion-ion plasma, the two ion species are identical (same masses, same temperature, etc.), resulting in identical diffusions. As there is no need to accelerate the slower species and retard the faster species, the plasma potential is equal to the potential at the wall enclosing the plasma. The floating potential is equal to the plasma potential since the collected ion currents are equal for a bias set to the plasma potential. In the case of the helicon reactor with grounded walls, the plasma and floating potential should be zero.

\section{Radial Profiles}

The radial profiles of the plasma and floating potential (figure 5.4a), as well as the electron temperature (figure 5.4b), are shown in figure 5.4 for argon, oxygen and sulfur hexafluoride. The gas pressure is set to $p=0.5 \mathrm{~m}$ Torr with the plasma on for the three gases. The applied power is $P=500 \mathrm{~W}$ and the magnetic field $B=120 \mathrm{G}$ for a coil current of $I=8.5$ A. The measurements were done with a filament probe placed in the diffusion chamber and connected to the Scientific Systems box. The automated analysis of the I-V characteristics, however, could not be used as the plasma parameters were meaningless. The analysis was done using lab-made programs where proper analysis parameters could be chosen.

The first interesting feature is the fact that the plasma potential as a function of radius is roughly constant. The situation is thus different from a non-magnetized electropositive plasma, where the fast diffusion of electrons creates an equilibrium with a decreasing plasma potential as a function of radius. The constant plasma potential, therefore, shows that electrons are magnetically confined.

The electron temperature profile for $r \leq 4 \mathrm{~cm}$ shows that the electron heating occurs at the center of the diffusion chamber, which roughly corresponds to the radius of the source chamber above the diffusion chamber. This confirms that the electron diffusion along the axis of the helicon reactor is fast, and that some heating occurs at the center of the diffusion chamber because of helicon waves propagating along the magnetic field lines. What is seen for $r \geq 4 \mathrm{~cm}$ is an electron temperature decrease, as they are confined by the magnetic field without any heating mechanism. This is similar to what was found for the PEGAES thruster prototype I in section 4.4, where the influence of the gas on the electron temperature at the center was discussed. Electrons are able to diffuse slowly across the magnetic field by collisions (elastic, ionizing, attaching, detaching) and thus lose energy. Near the edge of the diffusion chamber, the plasma and floating potentials come together. In argon, the small potential difference $V_{p}-V_{f}$ is due to the low electron temperature, but cannot be zero as the electron current is still greater than the positive ion current. In oxygen and sulfur hexafluoride, the potential difference $V_{p}-V_{f}$ is close to zero since the plasma behaves as an ion-ion plasma (negligible electrons). As oxygen is a weaker electronegative gas than sulfur hexafluoride, the radius at which $V_{p}-V_{f}$ comes to zero is larger.

Contrary to what is expected for an ideal ion-ion plasma, the plasma near the edge does not have plasma and floating potentials equal to zero. The plasma potential at the center of the discharge is set by the high electron temperature since electrons diffuse normally along the magnetic field lines. The relatively high value of the plasma potential accelerates positive ions and retards electrons near the grounded wall so that charge losses on the wall are equal (quasi-neutrality). As electrons are confined and lost through attachment, the radial plasma potential stays constant. However, this constant plasma potential does not 


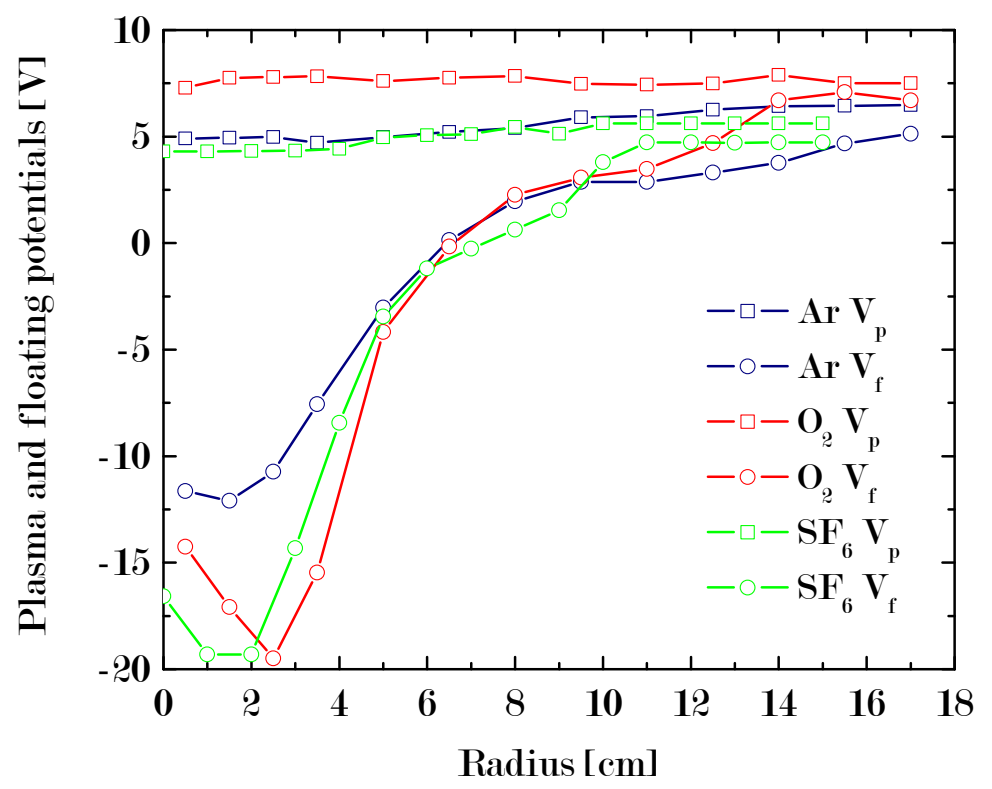

(a)

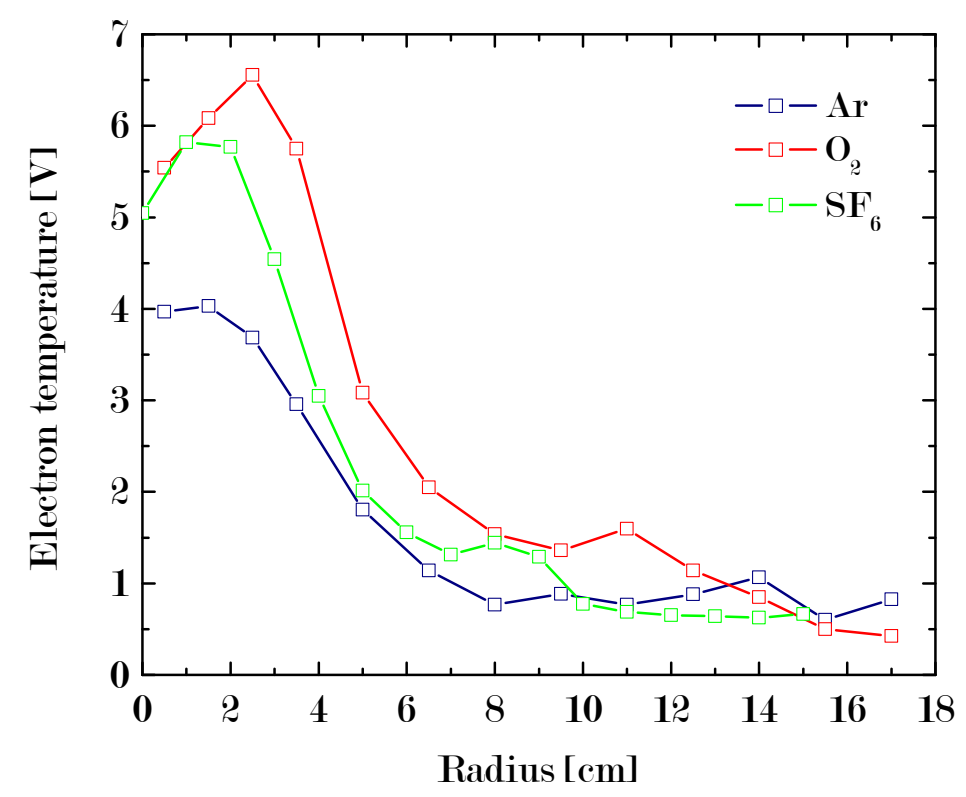

(b)

Figure 5.4: Plasma (square) and floating (circle) potentials (a) and electron temperature (b) as functions of radius for argon (blue), oxygen (red) and sulfur hexafluoride (green). Pressure of $p=0.5$ mTorr with plasma on for $P=500 \mathrm{~W}$ and $B=120 G$. 


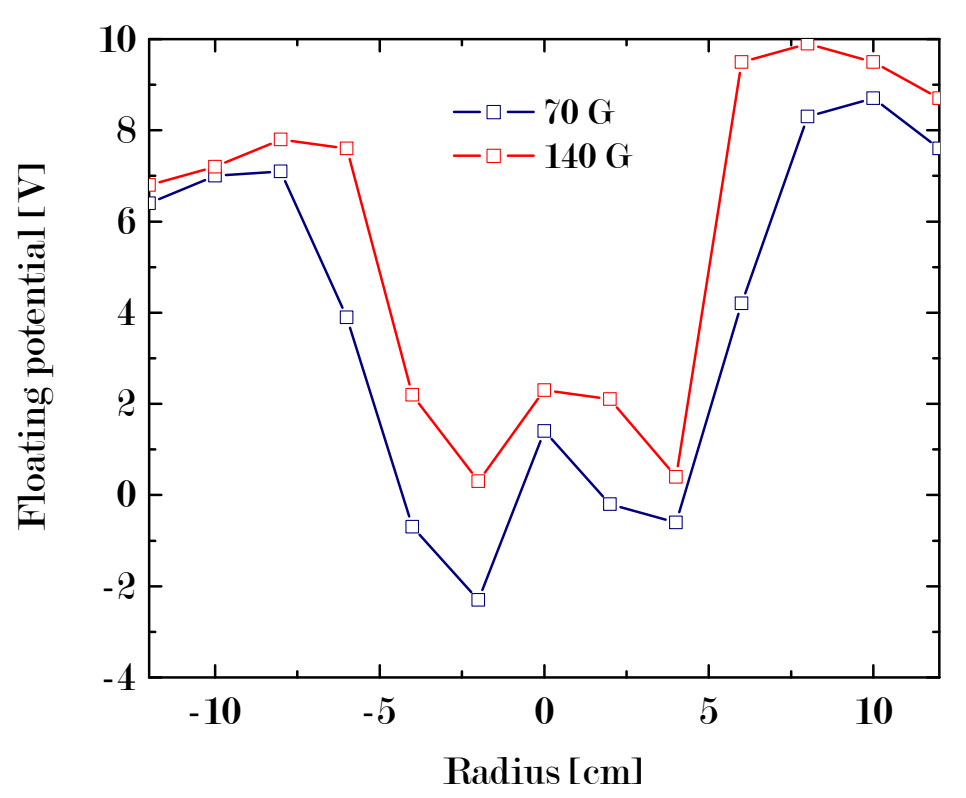

Figure 5.5: Floating potential as a function of position on the diffusion chamber diameter in oxygen for $B=70 G$ (blue) and $B=140 G$ (red). The pressure is set to $p=2.6$ mTorr, and the applied power to $P=500 \mathrm{~W}$.

correspond to the potential drop where electrons dominate the plasma equilibrium, with a value around $5 T_{e}$. Therefore, it is an intermediate value. This may be a consequence of the deposition of an insulating layer on the helicon chamber walls. These no longer behave as grounded electrodes, but as dielectric surfaces. The current distribution on the walls is thus complex and cannot be described easily.

\section{Floating Potential Symmetry}

The floating potential profiles in figure 5.4 were done on a radius of the helicon diffusion chamber, assuming that the plasma parameters were independent on the cylindrical angle. In order to look into that, the floating potential was measured with a filament probe, connected to a voltmeter, over the diameter of the diffusion chamber. The profiles for two values of the magnetic field, $B=70 \mathrm{G}$ for a coil current of $I=5 \mathrm{~A}$ and $B=140 \mathrm{G}$ for $I=10 \mathrm{~A}$, are shown in figure 5.5 in oxygen for a pressure of $p=2.6 \mathrm{mTorr}$. The applied power was set to $500 \mathrm{~W}$. The floating potential profile as a function of radius is the same as described previously.

It can be seen that the floating potential profiles are not symmetrical around the center of the diffusion chamber. This may be due to the non-homogeneous electron heating in the source chamber. The design of a Boswell-type antenna, presented in figure 4.1 is highly asymmetrical. As a result, the electron density and temperature depend on the azimuthal angle. As the plasma diffuses into the diffusion chamber, it remains asymmetrical, hence the asymmetrical floating potential profiles. However, it should be noted that the measurements were done with the filament probe placed on one side of the diffusion chamber. As points are measured away from this side, the perturbation caused by the probe holder crossing the entire diameter becomes more important. This could also play a role in the asymmetry of the measured profiles.

The floating potential profile is shown for two values of the magnetic field, $70 \mathrm{G}$ and 
$140 \mathrm{G}$. In the central region $(|r|<5 \mathrm{~cm})$, the floating potential increases with the magnetic field, but the profile is unchanged. The structure of the core is thus unchanged. However, in the periphery $(|r|>5 \mathrm{~cm})$, the influence of the magnetic field can be seen as the floating potential increases more rapidly for the higher value of the magnetic field. This shows that as expected, the electron confinement is obtained at a smaller radius for a greater magnetic field.

\subsubsection{RFEA Measurements}

A retarding field energy analyzer is used to investigate the energy distributions and the measured plasma potential for positive and negative ions. Specific grid and collector biases are needed for the case of negative ions, as well as specific conditions.

Retarding field energy analyzer measurements were done in argon for positive ions, and in sulfur hexafluoride for positive and negative ions.

\section{Positive and Negative Ions}

In a weakly electronegative plasma, the plasma equilibrium is dominated by electrons. This results in a sheath at the wall similar to that of an electropositive plasma, where the electrons are partially confined in the plasma due to the potential drop in the sheath. Negative ions, therefore, are all the more confined to the plasma. In such a case, retarding field energy analyzer measurements cannot be done for negative ions since they cannot be extracted. Pulsed plasmas, however, have been used to extract negative ions [40]. In an ion-ion plasma, however, the negative ions dominate the plasma equilibrium as there are no electrons. Retarding field energy analyzer measurements can thus be done with a negative collected current consisting of negative ions.

The setup of the analyzer for positive ions in the case of an electron-positive ions plasma was presented in section 2.2.3. Since there are no fast electrons to repel, the reject grid bias does not need to be as high as for an electropositive plasma. With experiments where the reject grid bias was varied, it was actually seen that too high a reject grid bias perturbs the measurements, and should be avoided. The reject grid bias is thus set to $-30 \mathrm{~V}$ for the measurement of positive ions in ion-ion plasmas. As the plasma potential in the ion-ion plasma resulting from the magnetic filtering was found to be around $5-10 \mathrm{~V}$, the bias range for the discriminating grid is fairly small (typically $-10 \mathrm{~V}$ to $40 \mathrm{~V}$ ) and centered around a value closer to zero.

As negative ions are oppositely charged compared to positive ions, the grid and collector biases in the retarding field energy analyzer need to be adjusted to first repel positive ions, then discriminate negative ions, and finally collect negative ions. The corresponding grid and collector biases are shown in figure 5.6. The influence of the reject grid bias was also studied and showed that for biases higher than $20 \mathrm{~V}$, the negative ion current collection is perturbed. Similarly to the positive ion measurement in ion-ion plasmas, the bias range for the discriminating grid is small and centered near zero. The potential difference between the secondary grid and the collector is kept unchanged as electrons can also be emitted from the collector.

\section{Results}

The position of the peak in the derivative corresponds to the plasma potential. It was thus investigated, as well as the saturation current when all particles are collected. It should 


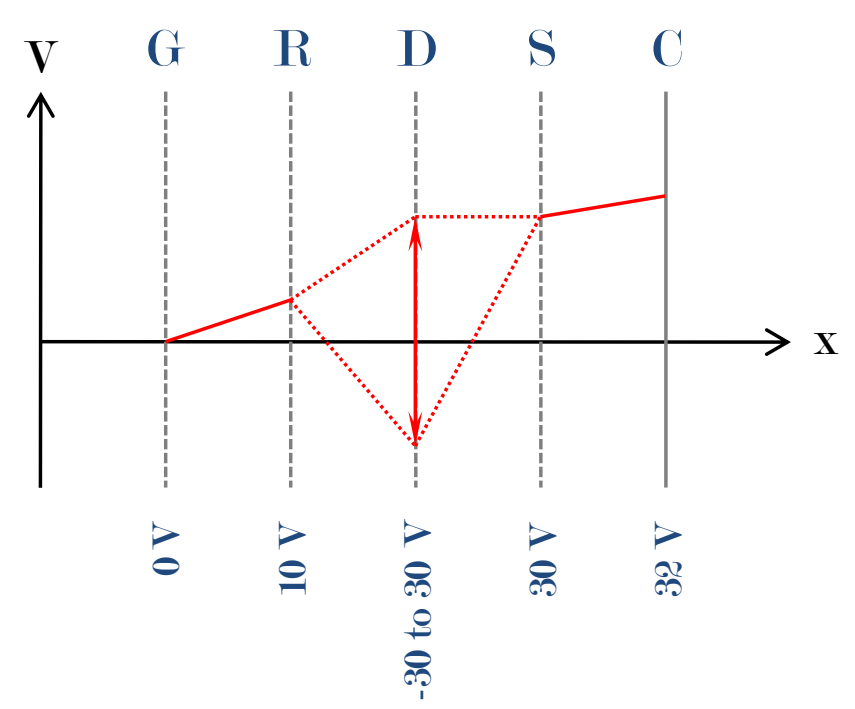

Figure 5.6: Schematics of the retarding field energy analyzer, and grid and collector biases in the case of a negative ion measurement.

be noted that the collected current is proportional to the voltage across the resistance in the measuring circuit (figure 2.8 in section 2.2.4).

Retarding field energy analyzer measurements for positive ions were first done in argon as it is an electropositive gas. These can then be compared to measurements for positive ions in sulfur hexafluoride, an electronegative gas. Finally, the obtention of measurements for negative ions in sulfur hexafluoride shows that the plasma at the periphery is indeed an ion-ion plasma.

Positive Ions in Argon A characteristic profile for the current collection (figure 5.7a) and the corresponding derivative (figure 5.7b) for the measurements of positive ions in argon is shown in figure 5.7. The gas pressure was set to $p=0.5 \mathrm{mTorr}$ and the magnetic field to $B=50 \mathrm{G}$ for a coil current of $I=3.5 \mathrm{~A}$. The applied power was $P=500 \mathrm{~W}$.

Three gas pressures were investigated: $p \in\{0.5,1,3\}$ mTorr. The position of the peak was found to be weakly sensitive to the gas pressure with an average around 7-9 V. This value is consistent with the plasma potential measurements shown in figure 5.4 showing a plasma potential of $7 \mathrm{~V}$. As to the saturation current, it is a slowly decreasing function of gas pressure, from an average voltage of $0.14 \mathrm{~V}$ at $p=0.5 \mathrm{~m}$ Torr to an average voltage of $0.11 \mathrm{~V}$ at $p=3 \mathrm{~m}$ Torr. This decrease of the saturation current as the pressure is increased may be due to edge-to-center density ratio $h$, which is a decreasing function of pressure.

Positive Ions in Sulfur Hexafluoride Figure 5.8 shows a characteristic profile for the current collection (figure 5.8a) and the corresponding derivative (figure 5.8b) for positive ions in sulfur hexafluoride. At an applied power of $P=500 \mathrm{~W}$, the pressure was set to $p=0.5 \mathrm{~m}$ Torr and the magnetic field to $B=45 \mathrm{G}$ for a coil current of $I=3 \mathrm{~A}$.

For a pressure of $p=0.5$ mTorr, the average position of the peak was found around $-5 \mathrm{~V}$ and the average saturation current around a voltage of $0.3 \mathrm{~V}$. From the plasma potential measured in figure 5.4, the expected value is around $5 \mathrm{~V}$. In the case of an ideal ion-ion plasma composed of ions with the same masses and temperatures, the expected value is $0 \mathrm{~V}$. It can be seen that the measured peak position corresponds to neither cases, 


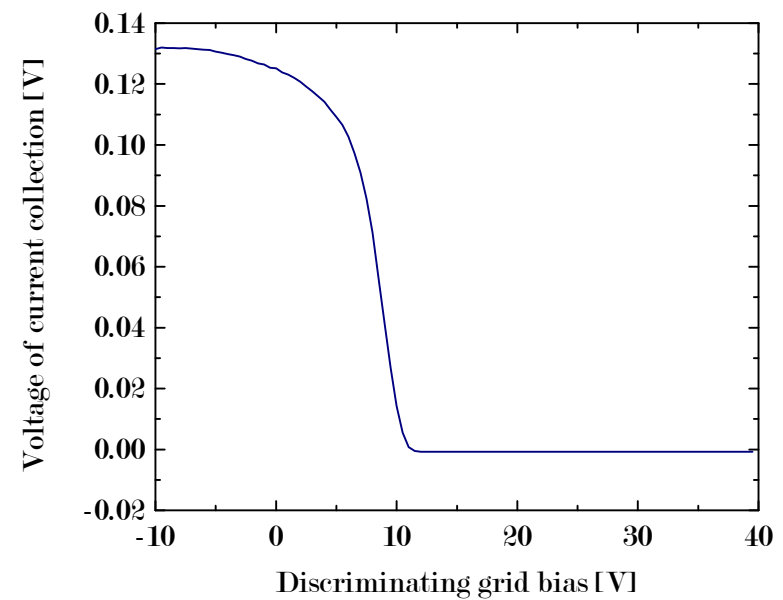

(a)

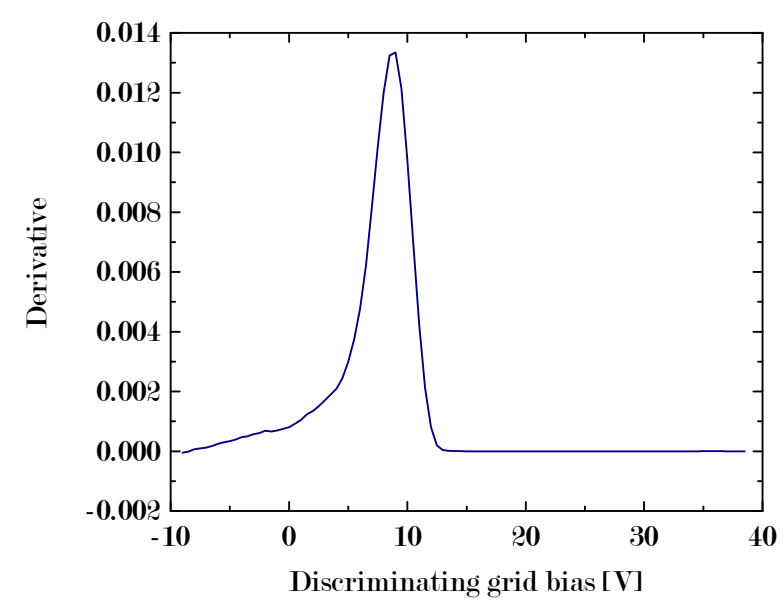

(b)

Figure 5.7: Voltage (a) corresponding to the collected current and derivative (b) as a function of discriminating grid bias for a measure of positive ions in argon. The pressure is set to $p=0.5$ mTorr, the magnetic field to $B=50 \mathrm{G}$, and the applied power $P=500 \mathrm{~W}$.

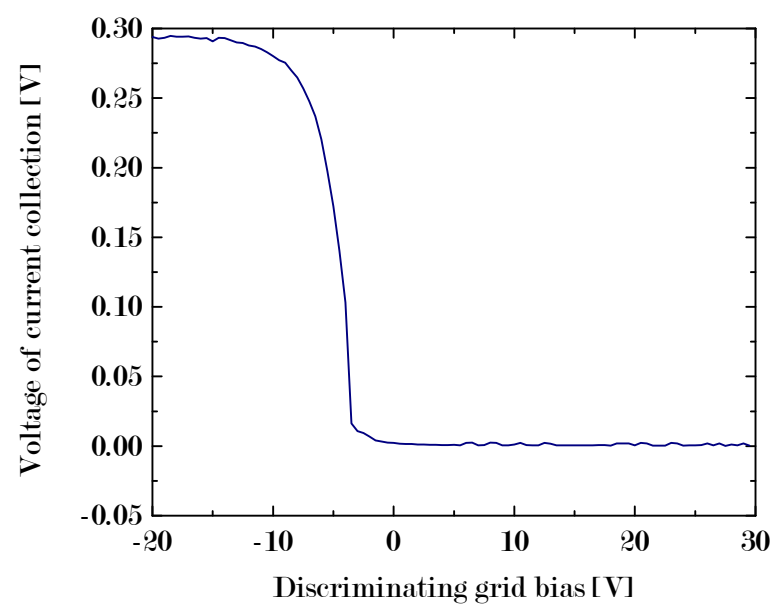

(a)

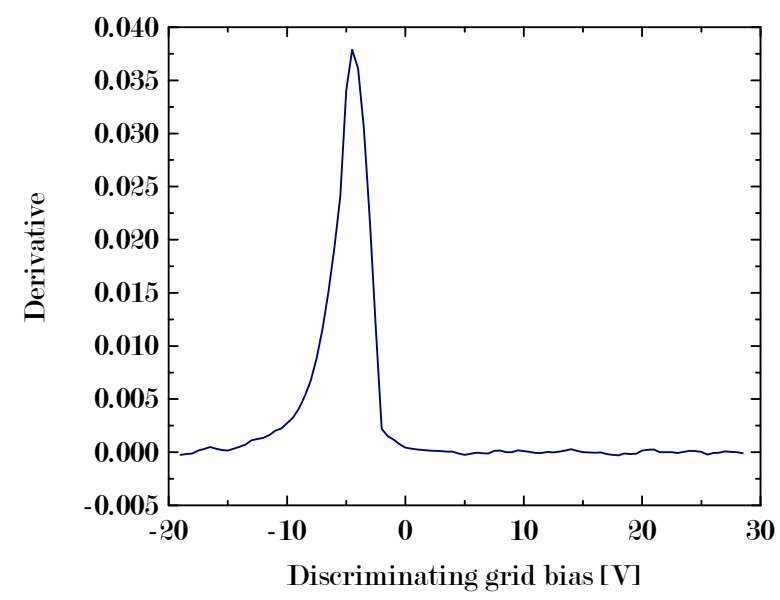

(b)

Figure 5.8: Voltage (a) corresponding to the collected current and derivative (b) as a function of discriminating grid bias for a measure of positive ions in sulfur hexafluoride. The pressure is set to $p=0.5$ mTorr, the magnetic field to $B=45 G$, and the applied power to $P=500 \mathrm{~W}$. 


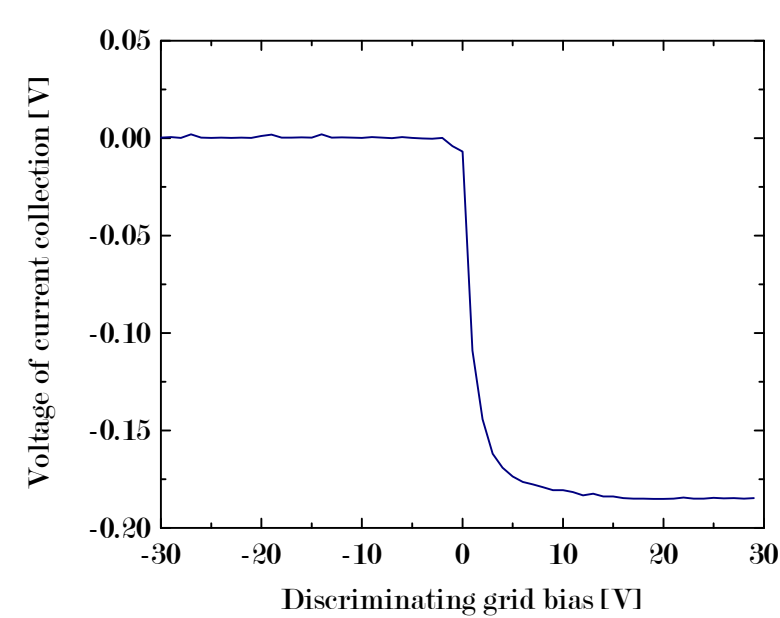

(a)

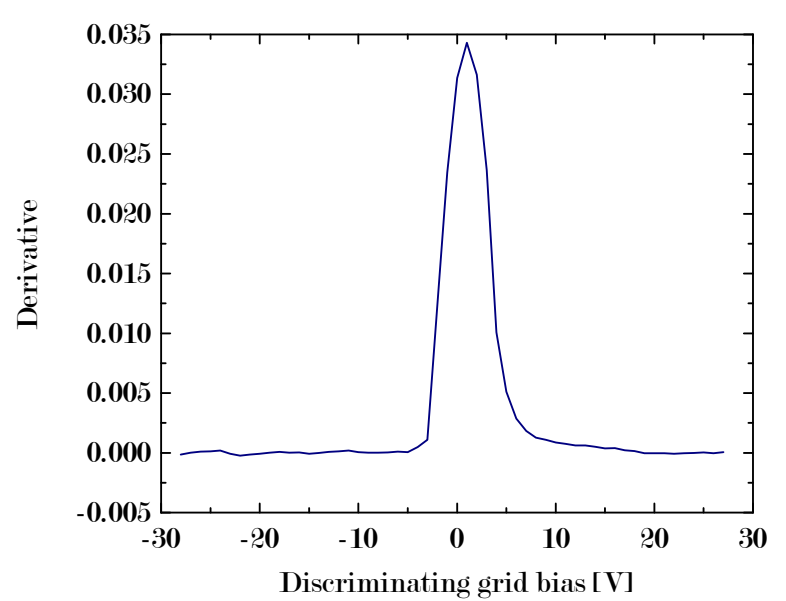

(b)

Figure 5.9: Voltage (a) corresponding to the collected current and derivative (b) as a function of discriminating grid bias for a measure of negative ions in sulfur hexafluoride. The pressure is set to $p=0.5 \mathrm{mTorr}$, the magnetic field to $B=45 \mathrm{G}$, and the applied power to $P=500 \mathrm{~W}$.

but shows a shift toward negative values. Since the chamber walls are deposited, this negative value could be explained by a charging effect on the walls and the probe. As positive charges are extracted, the plasma near the probe becomes negative since the positive ion current extracted is not negligible.

Negative Ions in Sulfur Hexafluoride A characteristic profile for the current collection (figure 5.9a) and the corresponding derivative (figure 5.9b) for the measurements of negative ions in sulfur hexafluoride is shown in figure 5.9. The gas pressure is set to $p=0.5$ mTorr, the magnetic field to $B=45 \mathrm{G}$ for a coil current of $I=3 \mathrm{~A}$, and the applied power to $P=500 \mathrm{~W}$.

For a pressure of $p=0.5$ mTorr, the average peak position was found to be around 2-3 V. This value is roughly the opposite of the one for positive ions for the same plasma conditions. Similarly to the positive ion case, this positive value could be the result of the plasma becoming positively charged as a non-negligible negative ion current is extracted. This would mean that the plasma potential of the unperturbed ion-ion plasma is zero, corresponding to the case of an ideal ion-ion plasma.

As the saturation current corresponds to a voltage of $0.15 \mathrm{~V}$ on average, it is half of the one for positive ions. This could be a consequence of the different average masses of positive and negative ions in sulfur hexafluoride. The smaller saturation voltage corresponds to a higher mass. In this case, however, the floating potential is no longer zero, as will be shown in chapter 7 . As figure 5.3 shows symmetrical currents, a possible effect is the detachment of negative ions in the retarding field energy analyzer.

\subsubsection{Electronegativity Measurements}

The obtention of an ion-ion plasma in the periphery of the electronegative discharge was shown in the previous sections with I-V characteristics, plasma and floating potential, and retarding field energy analyzer profiles. Here, the negative ion fraction is quantified as a 


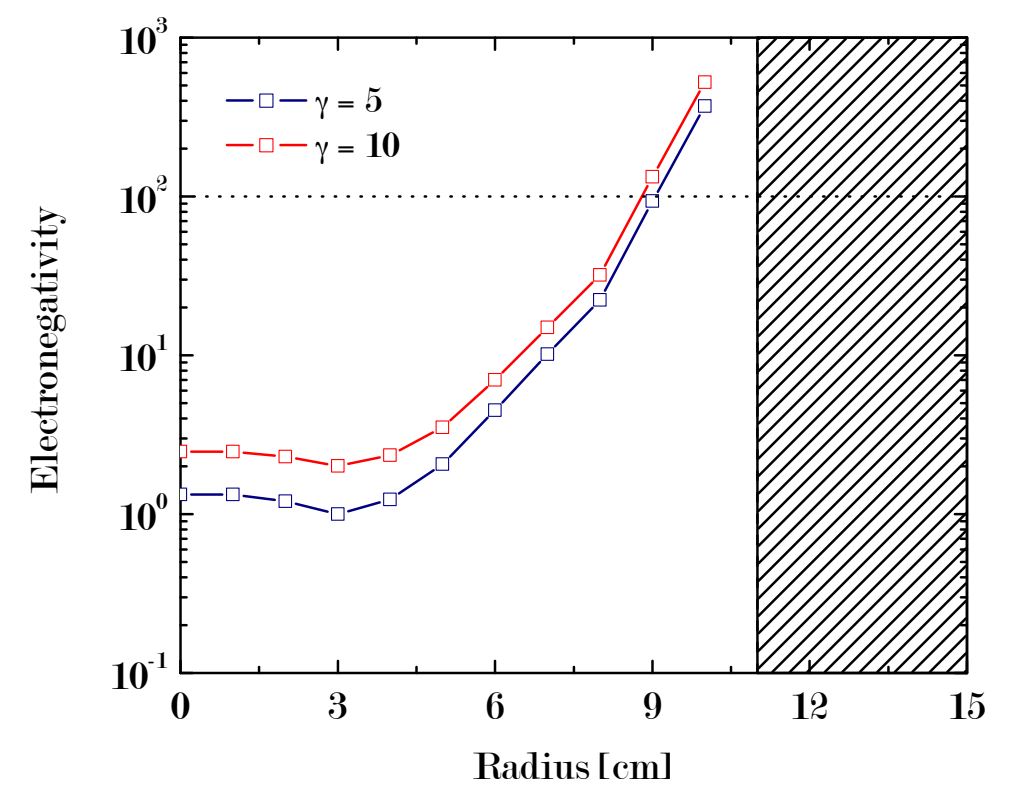

Figure 5.10: Electronegativity as a function of radius in sulfur hexafluoride for $\gamma=5$ (blue) and $\gamma=10$ (red). The shaded region corresponds to the region where the ion-ion plasma is obtained $(r \geq 11 \mathrm{~cm})$, and the dotted line to the maximum value of $\alpha$ valid for the technique. Pressure $p=0.5$ mTorr with plasma on, with an applied power of $P=500 \mathrm{~W}$ and a magnetic field of $B=120 \mathrm{G}$.

function of radius.

The radial profile of $\alpha$ in sulfur hexafluoride is shown in figure 5.10. The plasma conditions are $p=0.5$ mTorr with plasma on, for an applied power of $P=500 \mathrm{~W}$ and a magnetic field of $B=120 \mathrm{G}$ (coil current of $I=8.5 \mathrm{~A}$ ). Two typical value of $\gamma=T_{e} / T_{-}$ were chosen: $\gamma \in\{5,10\}$. The electrostatic probe technique is only valid for $\alpha<100$, limit showed as a dotted line. However, the increase of the negative ion fraction along the radius is seen. As the electronegativity becomes greater than $\alpha=100$, the curve is seen to increase exponentially as it reaches the shaded region. Values beyond this asymptote are not meaningful as the electron density becomes negligible. This confirms that the electronegative plasma behaves as an ion-ion plasma at the periphery of the discharge.

As described previously, the negative ion production is rather small at the center of the discharge $(r<4 \mathrm{~cm})$ where the electron temperature is high, as can be seen with the small value of the electronegativity. At the periphery where the electron temperature drops significantly, the electronegativity shows an exponential behavior. The electronegativity of $\alpha=100$ is reached at $r=9 \mathrm{~cm}$, which could mean that electrons are negligible beyond this point. However, looking at the I-V characteristic in sulfur hexafluoride at $r=10 \mathrm{~cm}$ in figure 5.3 suggests that the negative current is an intermediate current where neither the electrons nor the negative ions dominate. In this case, the negative current is roughly two times greater than the positive current.

\subsection{PEGASES Prototype I}

The design of the first PEGASES thruster prototype was based on the helicon reactor since ion-ion plasmas were obtained in this reactor. Although some modifications were made, 
among which the fact that it consists of a double helicon, the ion-ion plasma formation should be similar.

However, I-V characteristic profiles showed that the plasma is electron dominated in the whole thruster volume. Electronegativity measurements were done to understand the negative ion creation.

\subsubsection{I-V Characteristics}

Normalized I-V characteristics at $r=8.5 \mathrm{~cm}$ are showed in figure 5.11 in argon (figure 5.11a) and oxygen (figure 5.11b) for a magnetic field with a coil current $I=1.5 \mathrm{~A}$. This value of the radius corresponds to the outer edge of the extraction tube, while the magnetic field strength to the optimum value. Two gas flows, $20 \mathrm{sccm}$ and $50 \mathrm{sccm}$, and two applied powers, $300 \mathrm{~W}$ and $600 \mathrm{~W}$, were investigated.

The I-V characteristics in argon are highly asymmetrical as the electron current is greater than the positive ion current, even with a low temperature (see figure 4.13 in section 4.4). The I-V characteristics in oxygen are similar to that in argon, with a negative current greater than the positive ion current. An ion-ion plasma is not observed in the extraction tube of the PEGASES thruster prototype I.

\subsubsection{Electronegativity Measurements}

The electronegativity as a function of radius in oxygen is shown in figure 5.12. Two gas flows were considered, $20 \mathrm{sccm}$ (figure 5.12a) and $50 \mathrm{sccm}$ (figure 5.12b), as well as two applied powers, $300 \mathrm{~W}$ and $600 \mathrm{~W}$. The magnetic field is set with a coil current of $1.5 \mathrm{~A}$. The ratio of electron to negative ion temperature is set to the classical value of $\gamma=T_{e} / T_{-}=10$. The electronegativity can be seen to be roughly constant with values well below $\alpha=100$.

The electronegativity measurements show that negative ions are present in the plasma, with a density higher than that of the electrons. However, with an average value below $\alpha=5$, the negative ion fraction is too small for the electronegative plasma to behave as an ion-ion plasma, as showed by I-V characteristics. Although the conditions seem very similar to the helicon reactor (magnetic field configuration, electron temperature decrease, etc.), two differences could account for the non-obtention of an ion-ion plasma. The first difference is the magnetic field strength. The scaling factor between the radius of the diffusion chamber of the helicon reactor and that of the thruster cylinder is 7 . The magnetic field in the thruster, however, is only twice as strong as in the helicon reactor. Since similar pressures were considered, the magnetic field might be too weak to properly confine the electrons. The second difference is the neutral density distribution. In the helicon reactor, neutrals are introduced in the diffusion chamber, and diffuse into the source region. In the PEGASES thruster prototype I, the neutrals are injected in the source region, and diffuse into the extraction tubes. The consequence of this reversal is that neutral molecules are present where the electron temperature is low in the helicon reactor, whereas the extraction tubes of the PEGASES thruster prototype I are mostly fed with atoms produced by the dissociation of molecules by the high energy electrons in the source region of the thruster cylinder. Since the negative ion creation is only possible with molecules (dissociative attachment) in oxygen, the negative ion production is dramatically inefficient in the PEGASES thruster prototype I. 


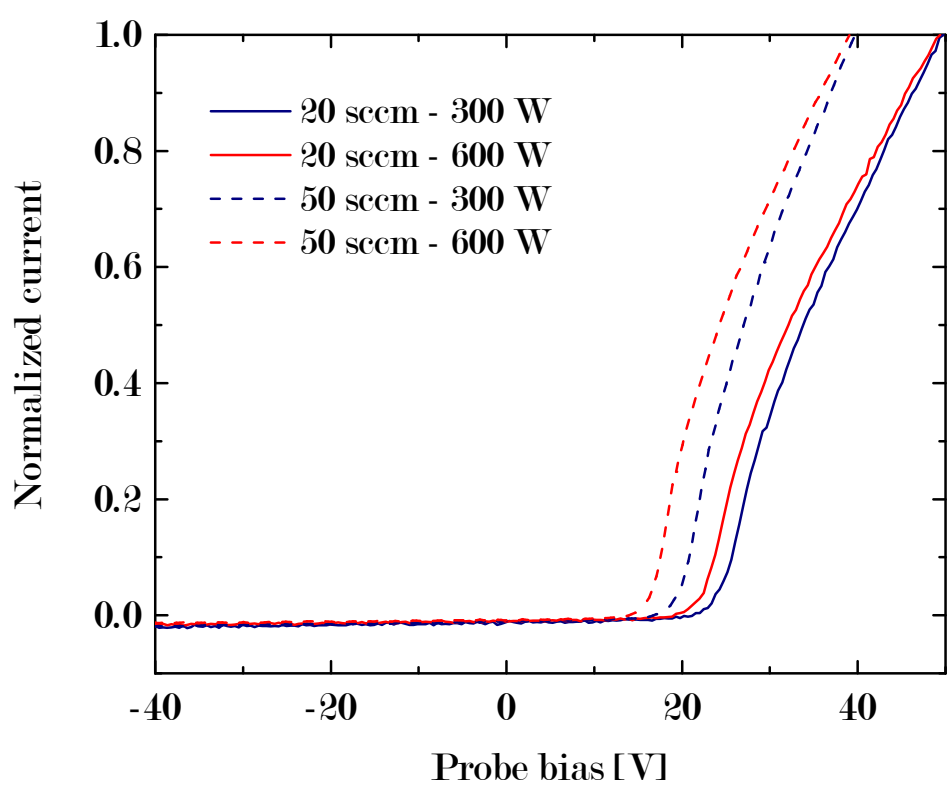

(a) $\mathrm{Ar}$

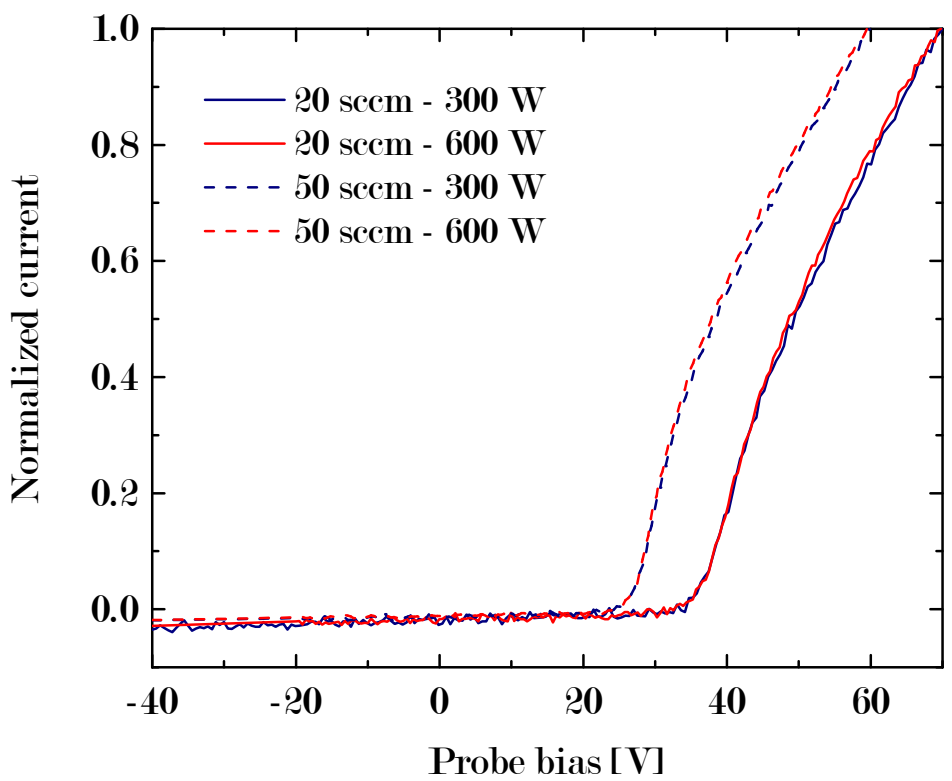

(b) $\mathrm{O}_{2}$

Figure 5.11: Normalized $I-V$ characteristics at $r=8.5 \mathrm{~cm}$ in argon (a) and oxygen (b) for a magnetic field with a coil current $I=1.5 \mathrm{~A}$. Gas flows of $20 \mathrm{sccm}$ (solid line) and $50 \mathrm{sccm}$ (dashed line), and applied powers of $300 \mathrm{~W}$ (blue) and $600 \mathrm{~W}$ (red) were used. 


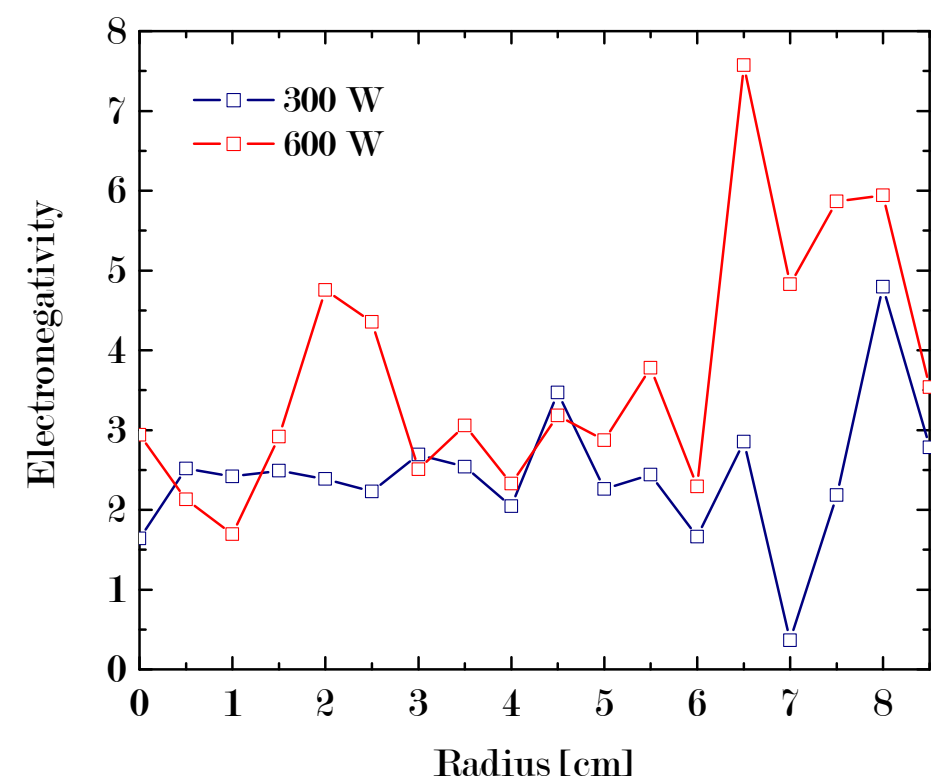

(a) $20 \mathrm{sccm}$

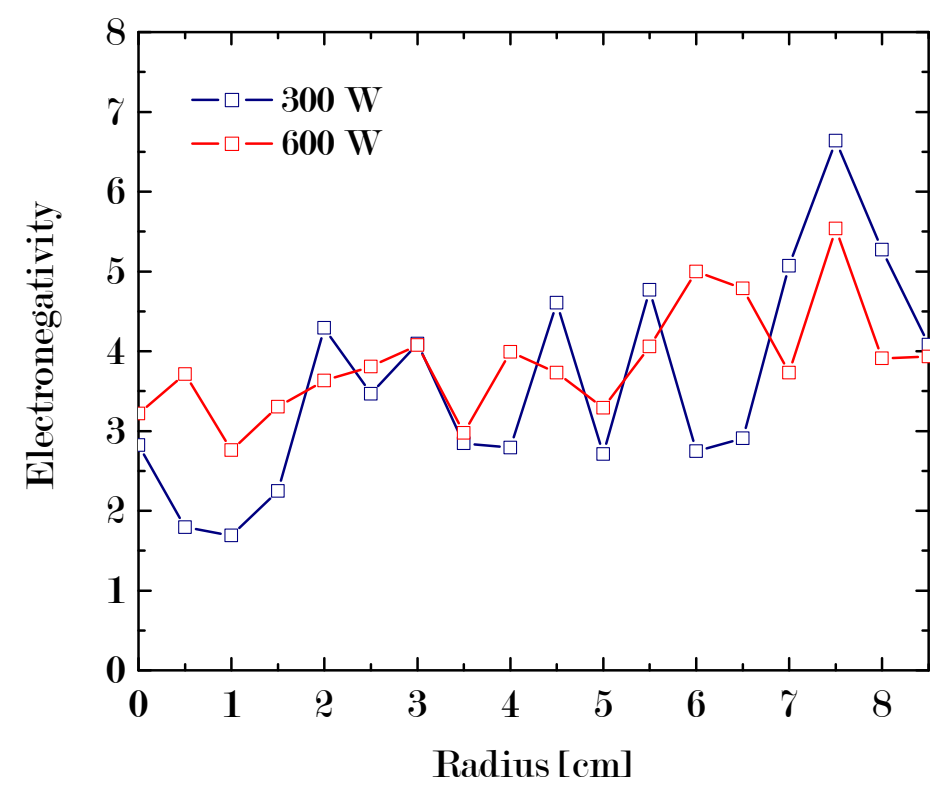

(b) $50 \mathrm{sccm}$

Figure 5.12: Radial profiles of the electronegativity in oxygen for $20 \mathrm{sccm}$ (a) and $50 \mathrm{sccm}$ (b). In each case, two applied powers were considered, $300 \mathrm{~W}$ (blue) and $600 \mathrm{~W}$ (red). The magnetic field is set with a coil current of $1.5 \mathrm{~A}$. The ratio of temperatures is set to $\gamma=10$. 


\subsection{Design Improvements}

The measurements in the first PEGASES thruster prototype showed that although some negative ions are produced, the average electronegativity below $\alpha=5$ is far too small. Following the two hypotheses that were proposed to account for the small electronegativity, two design improvements were done on the first PEGASES thruster prototype: the magnetic filtering is enhanced by adding permanent magnets, and the neutral injection is optimized.

\subsubsection{Enhanced Magnetic Filtering}

The magnetic filtering is enhanced by placing two permanent magnets on the side of an extraction tube, as described in section 3.2.3. The resulting magnetic field strength in the extraction tube is shown in figure 3.5, where it can be seen that it varies between $450 \mathrm{G}$ at the center and $2300 \mathrm{G}$ at the edge. The magnetic field created by the permanent magnets is thus much stronger than the one by the coils, between 150 and $300 \mathrm{G}$ for $I=3 \mathrm{~A}$. The influence of this stronger magnetic field on the positive ion current density and the mass efficiency was investigated.

\section{Positive Ion Current Density}

The positive ion current density measurements shown in figure 5.13 were done in argon (figure 5.13a) and oxygen (figure 5.13b) for an applied power of $P=300 \mathrm{~W}$. The magnetic field is varied with the coil current from -3 to $3 \mathrm{~A}$. The gas flow is varied from 7.2 to $115.2 \mathrm{sccm}$ in argon, and from 10 to $80 \mathrm{sccm}$ in oxygen. The planar probe was placed near the outer edge of the extraction tube, and thus after the magnetic filter created by the two permanent magnets. It should be noted that a plasma in oxygen for a gas flow of 5 sccm cannot be obtained, as was the case before.

The most important feature is the fact that the current density in argon becomes negligible compared to that in oxygen, except at very low pressure. The plasma in argon (electropositive) is composed of electrons and positive ions. Since the electrons are confined to the magnetic field lines, they cannot diffuse through the magnetic filter. The diffusion of positive ions, therefore, is severely reduced as quasi-neutrality needs to be fulfilled. In oxygen, quasi-neutrality can be fulfilled beyond the magnetic filter with positive and negative ions. The current density, however, is smaller by a factor of 4 compared to the situation without the permanent magnets. The magnetic electron filtering was thus enhanced. The reduced density is due to the fact that the negative ion density is relatively low in the core of the thruster cylinder.

\section{Mass Efficiency}

Figure 5.14 shows the mass efficiency in oxygen at high power $P=1.6 \mathrm{~kW}$ as a function of gas flow from 10 to $55 \mathrm{sccm}$. The magnetic field is set with the coil current $I=1.5 \mathrm{~A}$ which corresponds to the maximum of current density.

It can be seen that the positive ion flux creation is very efficient (near 70\%) at very low pressure, but drops quickly as the gas flow is increased. For instance, the mass efficiency is below $10 \%$ for gas flows higher than $40 \mathrm{sccm}$. The profile is similar to a $x^{-1}$ dependence with $x$ the gas flow. This means that even at high applied power, an increase in neutral injection does not result in an increase in positive ion flux. 


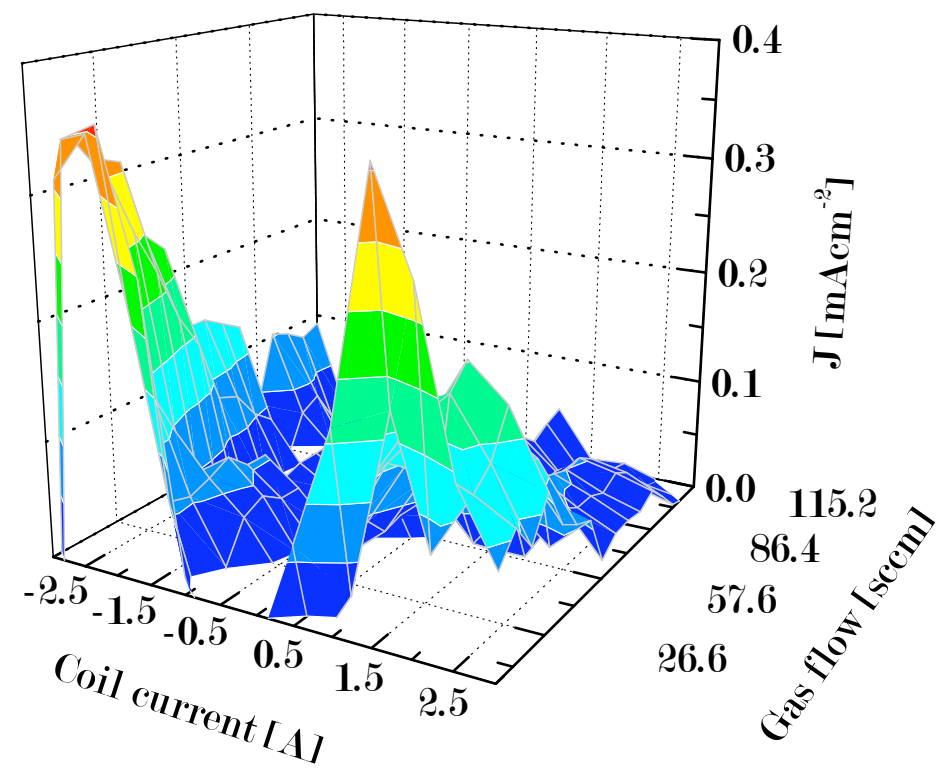

(a) $\mathrm{Ar}$

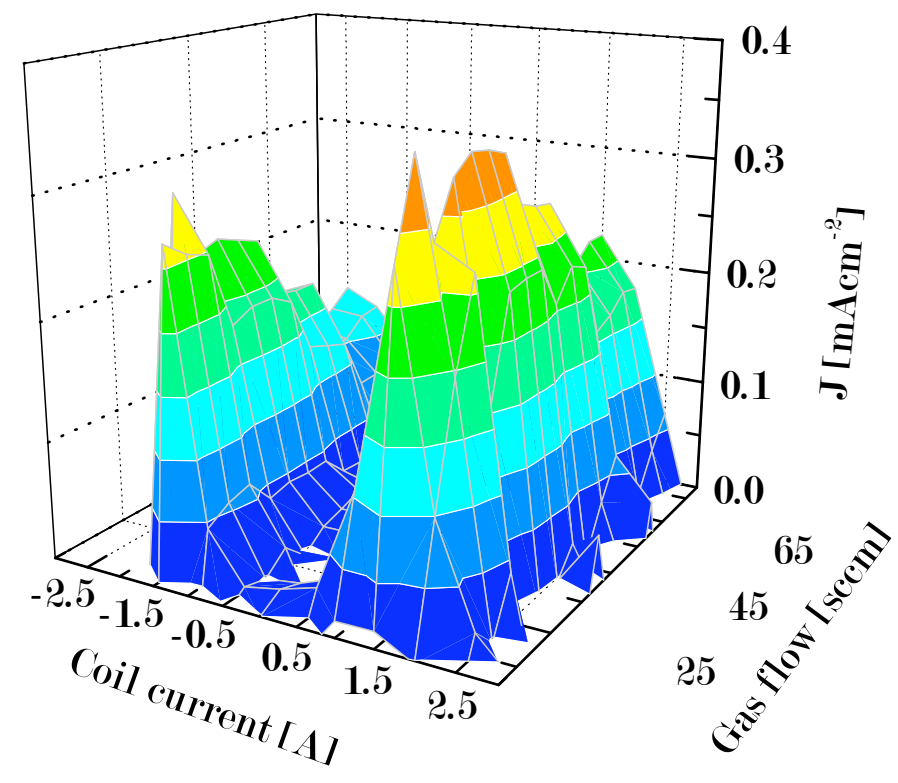

(b) $\mathrm{O}_{2}$

Figure 5.13: Current density in $\mathrm{mAcm}^{-2}$ for $P=300 \mathrm{~W}$ in argon (a) and oxygen (a) as a function of magnetic field, with the coil current from -3 to $3 \mathrm{~A}$, and gas flow, from 7.2 to $115.2 \mathrm{sccm}$ in argon and from 10 to $80 \mathrm{sccm}$ in oxygen. 


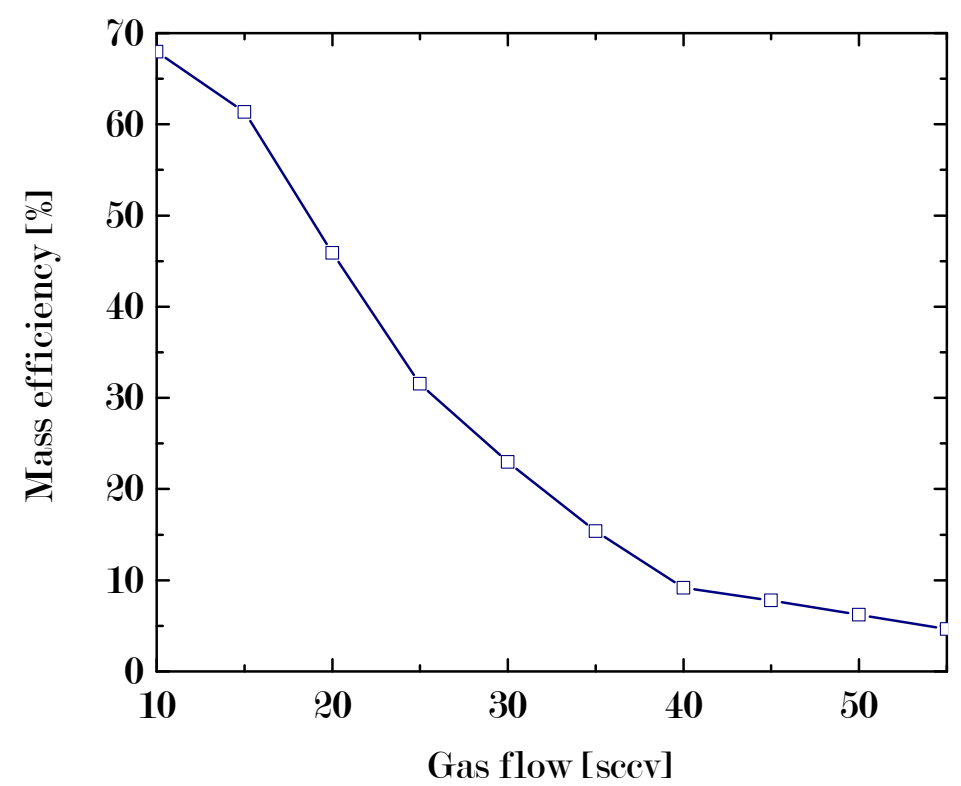

Figure 5.14: Mass efficiency in percent as a function of gas flow from 10 to $55 \mathrm{sccm}$ in oxygen at high power $P=1.6 \mathrm{~kW}$. The magnetic field is set with the coil current $I=1.5 \mathrm{~A}$.

\subsubsection{Optimized Neutral Injection}

As the enhanced magnetic filter showed little improvement, the second hypothesis is investigated. The issue is assumed to come from the fact that there are no neutral molecules where the electron temperature is low (extraction tube). As the attachment in oxygen is dissociative, the negative ion creation is limited. Only neutral atoms can be found in the extraction since neutral molecules travel through the high electron temperature core of the thruster.

In order to improve the design of the PEGASES thruster prototype I, a gas line is placed at the outer edge of an extraction of the PEGASES thruster, as can be seen in figure 3.8 in section 3.3.1. The gas flow can be controlled independently from the main gas flow in the middle of the thruster. In order to assess the role of the secondary gas injection, the permanent magnets from the previous attempt at improvement were removed. Figure 5.15 shows the I-V characteristics for three values of the secondary gas flow $(0,25$ and $50 \mathrm{sccm})$. The gas used for both injections is sulfur hexafluoride, with the main gas flow at $20 \mathrm{sccm}$. The applied power was set to $P=300 \mathrm{~W}$, and the magnetic field with a coil current of $I=1.5$ A.

The evolution of the I-V characteristics shows a transition from an electron dominated to a negative ion dominated plasma. These measurements were done by Lara Popelier during an internship (Master 2). For the intermediate gas flow of $25 \mathrm{sccm}$, the negative current is seen to be composed of electron and negative ion currents. Assuming that the neutral is divided in two equal parts between the two extraction tubes, the neutral gas flow from the main injection in an extraction tube is $10 \mathrm{sccm}$. As an ion-ion plasma is obtained for a secondary gas flow of $50 \mathrm{sccm}$, this shows that a factor of 5 is needed to attach most of the electrons created in the thruster cylinder and obtain negative ions.

The optimization of the neutral injection is the main aspect of a patent filed in November 2008 [27]. 


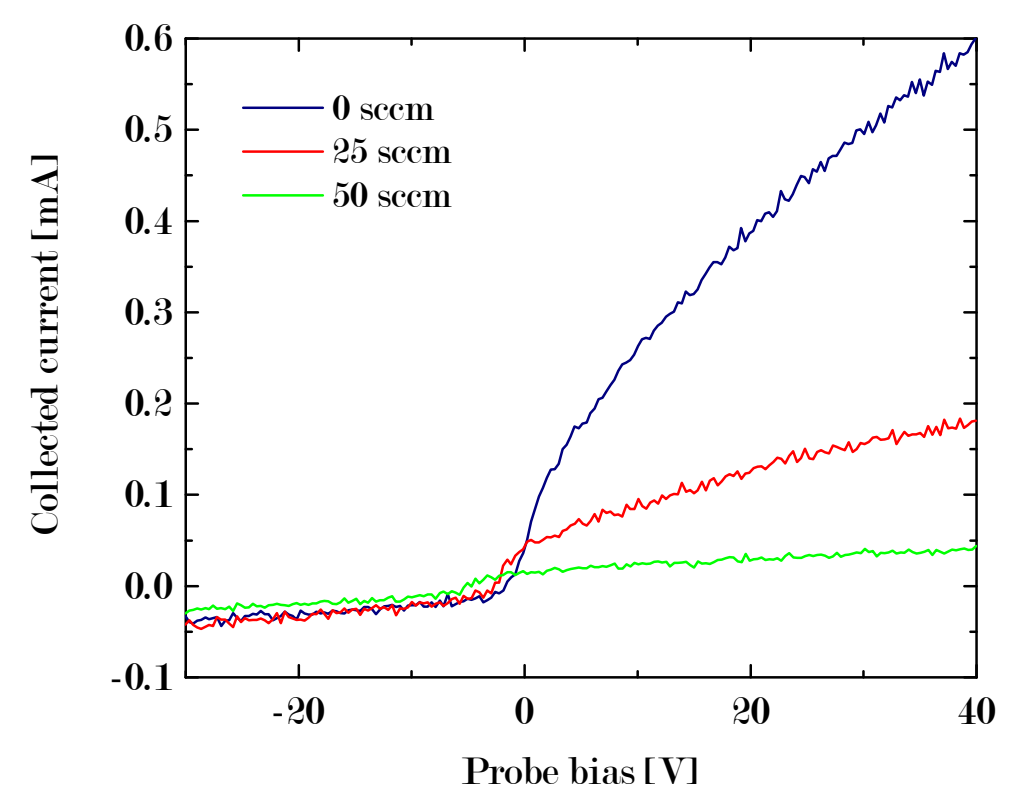

Figure 5.15: I-V characteristics in sulfur hexafluoride for three values of the secondary gas flow: 0 sccm (blue), $25 \mathrm{sccm}$ (red), and $50 \mathrm{sccm}$ (green). Applied power of $P=300 W$ for a main gas flow of 20 sccm and a magnetic field with coil current of $I=1.5 \mathrm{~A}$.

\subsection{Conclusions}

Contrary to the initial idea that the magnetic field created an ion-ion plasma by simply confining the electrons in a region to let positive and negative ions diffuse together with quasi-neutrality fulfilled, the effect of the magnetic field is twofold:

- The first effect of the magnetic field is indeed confinement of the electrons to let both ion species diffuse in another region (periphery in the case of a cylindrical geometry) where the ion-ion plasma is obtained. It was seen with the experiments on the PEGASES thruster prototype I that a high enough magnetic field strength is needed to properly confine the electrons. This optimum value depends on the size and pressure of the system.

- The second effect of the magnetic field is to create a profile in electron temperature. As electrons are confined along the magnetic field lines, they diffuse cross-field through collisions. Electrons, therefore, lose energy as they diffuse cross-field. This results in a strong electron temperature gradient, with high electron temperatures where electrons are heated (around $10 \mathrm{~V}$ ), and a low electron temperature elsewhere (around 2-3 V). Positive ions and electrons are thus created efficiently in the high electron temperature region through ionization collision, while the attachment of electrons on neutrals to obtain negative ions is efficient in the low electron temperature region.

However, an additional condition is needed to obtain an efficient electron attachment in the low electron temperature region. As attachment in oxygen is dissociative, neutral molecules and not atoms are needed. It was seen in the first PEGASES prototype that the injection of neutrals in the source region resulted in poor negative ion creation because molecules were dissociated in the thruster cylinder before reaching the extraction tubes. 
The first test of secondary neutral injection in the first PEGASES prototype showed that neutrals were the missing condition to the obtention of an ion-ion plasma. The neutral injection, moreover, can be optimized to control the creation of an ion-ion plasma. This resulted in a patent [68] on an evolution of the PEGASES thruster concept [23] that was filed as the role of neutrals was understood. 


\section{Part II}

\section{Models}





\section{Chapter 6}

\section{Magnetic Electron Filtering Stage - Theory}

\section{Contents}

6.1 Franklin Fluid Model in One Dimension . . . . . . . . . . . 107

6.2 Fluid Model Including End Losses . . . . . . . . . . . . . 110

6.2.1 Limitations of a 1D System . . . . . . . . . . . . . . 110

6.2 .2 Electron End Loss . . . . . . . . . . . . . . . . . . . . 111

6.2.3 Reformulated Model with End Loss . . . . . . . . . . . . . . . . 113

6.2.4 Results Using the Reformulated Model . . . . . . . . . . . . . . 116

6.3 Analytic Approximation . . . . . . . . . . . . 124

6.3 .1 Analytic Model . . . . . . . . . . . . . . . . . . . . . 124

6.3.2 Comparison with Numerics, Parameter Scaling . . . . . . . . . 130

6.4 Model Limitations . . . . . . . . . . . . . . . . 133

6.4.1 Symmetrical Ion-Ion Plasma . . . . . . . . . . . . . . . 133

6.4.2 Gas Electronegativity . . . . . . . . . . . . . . . 134

6.4.3 Solutions with $n_{e}(R) \neq 0 \ldots \ldots \ldots \ldots$

6.4.4 Constant $T_{e} \ldots \ldots \ldots \ldots \ldots \ldots \ldots$

6.4.5 Non-Uniform Neutral Density . . . . . . . . . . . . . . . 136

\subsection{Franklin Fluid Model in One Dimension}

Franklin and Snell studied the effect of negative ions on the positive column in a magnetic field at low pressure [69]. In an electropositive plasma (no negative ions), the magnetic field is known to reduce the electron diffusion, and in some cases to the extent that the positive ions become the more mobile of the two species [70, 71]. Adding negative ions to such a plasma was expected to modify the diffusion of the species even further since quasi-neutrality could be fulfilled without electrons.

Franklin and Snell [69] considered a one-dimensional (1D) fluid model of an oxygenlike discharge with three species (electrons, positive ions and negative ions) in either 
rectangular or cylindrical coordinates. The electrons are assumed to be magnetized while the ions are assumed to be unmagnetized. The ratio of masses between electrons and ions is such $\left(\sim 10^{5}\right)$ that the ion Larmor radius $r_{\mathrm{ci}}$ is a lot bigger than the electron Larmor radius $r_{\text {ce. }}$. It is then possible to choose a value of the magnetic field and a length for the system $R$ with

$$
r_{\mathrm{ce}}<R<r_{\mathrm{ci}}
$$

The reverse goes for the cyclotron frequencies $\left(\omega_{\text {ce }}\right.$ and $\left.\omega_{\text {ci }}\right)$ and the collision frequencies $\left(\nu_{e}\right.$ and $\left.\nu_{i}\right)$ :

$$
\omega_{\mathrm{ce}}>\nu_{e} \text { and } \omega_{\mathrm{ci}}<\nu_{i}
$$

Two cases were considered: detachment dominated case and recombination (electronion) dominated case. In the detachment dominated case for a rectangular geometry, the equations are the following

$$
\begin{gathered}
\nabla\left(n_{e} v_{e}\right)=\left(\nu_{\mathrm{iz}}-\nu_{\mathrm{att}}\right) n_{e}+\nu_{\mathrm{det}} n_{-} \\
\nabla\left(n_{+} v_{+}\right)=\nu_{\mathrm{iz}} n_{e} \\
\nabla\left(n_{-} v_{-}\right)=\nu_{\mathrm{att}} n_{e}-\nu_{\mathrm{det}} n_{-} \\
m_{e}\left(\nu_{\mathrm{att}}+\nu_{e}\right) n_{e} \mathbf{v}_{\mathbf{e}}+e n_{e} \mathbf{v}_{\mathbf{e}} \times \mathbf{B}_{\mathbf{0}}-e n_{e} \boldsymbol{\nabla} \varphi+e T_{e} \boldsymbol{\nabla} n_{e}=0 \\
e T_{+} \nabla n_{+}+e n_{+} \nabla \varphi+m_{+} \nabla\left(n_{+} v_{+} v_{+}\right)=0 \\
e T_{-} \nabla n_{-}-e n_{-} \nabla \varphi+m_{-} \nabla\left(n_{-} v_{-} v_{-}\right)=0 .
\end{gathered}
$$

In this case, the studied parameters were $\alpha_{F}=\nu_{\text {att }} / \nu_{\mathrm{iz}}, \beta_{F}=\nu_{\mathrm{det}} / \nu_{\mathrm{iz}}$ and $\delta_{F}$ a magnetic field parameter with the frequencies and reaction rates described in section 2.1.1. It can be seen in (6.7) and (6.8) that the ions are assumed collisionless.

Equation (6.6) is a vector equation, but can be easily written as a scalar one, and gives the expression of the magnetic field parameter

$$
\delta_{F}=\left(1+\frac{\nu_{e}}{Z}\right) \frac{m_{e}}{m_{+}}\left(1+\frac{\omega_{\mathrm{ce}}^{2}}{\left(Z+\nu_{e}\right)^{2}}\right) .
$$

It can be seen that $\delta_{F}$ is a function of the ratio of electron cyclotron frequency to electron collision frequency.

The initial conditions at $x=0$ are $\varphi=0, v_{e,+,-}=0, n_{e}=n_{e 0}, n_{-}=n_{-0}$ and $n_{+}=n_{e 0}+n_{-0}$. The boundary conditions at the wall $x=x_{w}$ are

$$
\nabla \varphi \rightarrow \infty \text { and } v_{-}=0, \text { or } n_{e}=0
$$

For a given $n_{-0} / n_{e 0}$, the computations give a relation between $\alpha_{F}, \beta_{F}$ and $\delta_{F}$. Figure 6.1 shows the normalized densities (figure 6.1a) and fluxes (figure 6.1b) for a choice of parameters: $n_{-0} / n_{e 0}=3, \delta_{F}=10, \beta_{F}=0.3$ and $\alpha_{F}=1.263$. This figure represents a typical result for the model. The boundary condition $n_{e}\left(x_{w}\right)=0$ is fulfilled when $\delta_{F}>1$, which means, as anticipated, that there is a minimum value of the magnetic field to obtain an electron confinement. The negative ion flux at the wall is zero, which means that the negative ions are confined to the center of the discharge. Since this is a 1D fluid model,

$$
\Gamma_{+}=\Gamma_{e}+\Gamma_{-}
$$




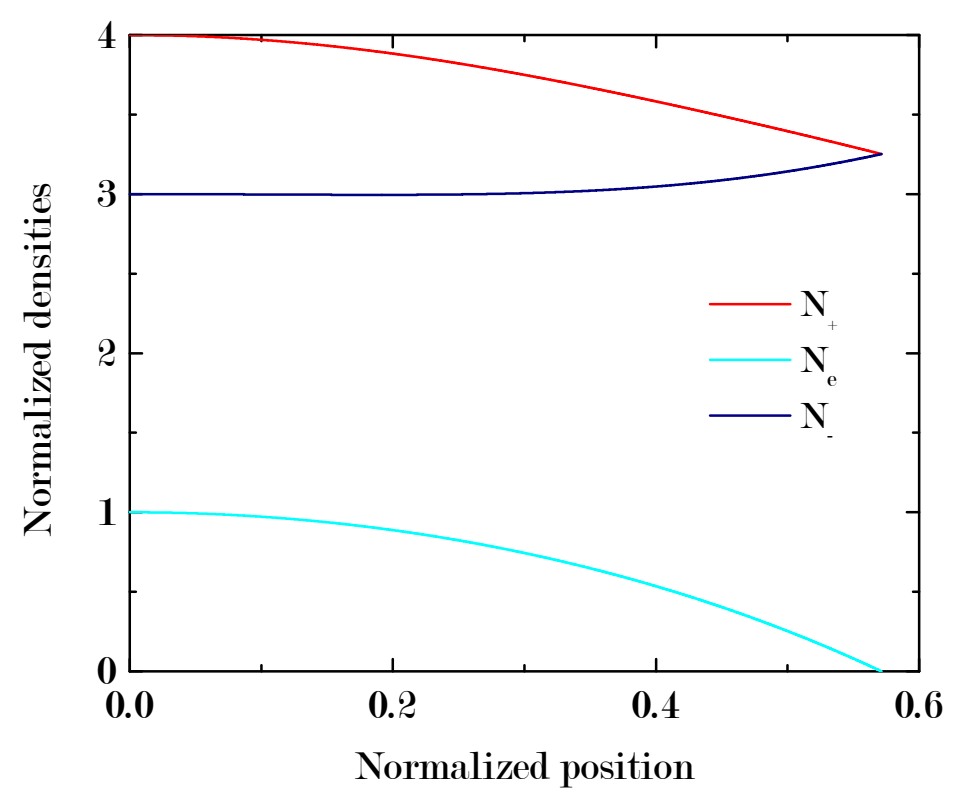

(a)

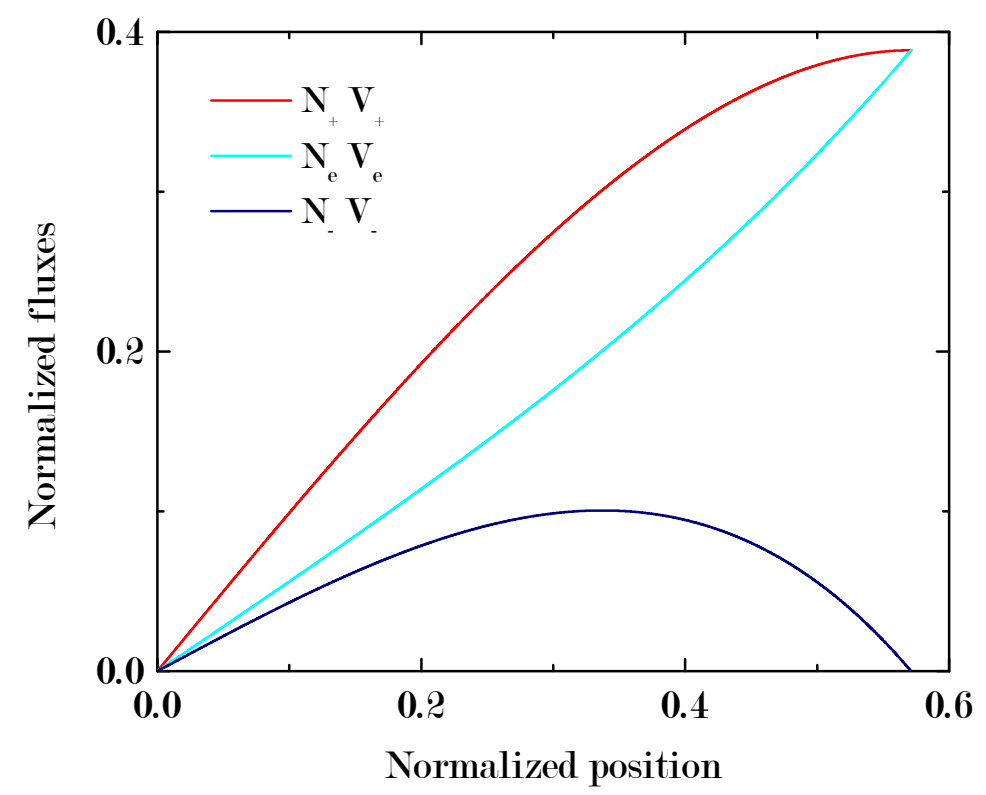

(b)

Figure 6.1: Normalized densities (a) and normalized fluxes (b) as a function of normalized position for $n_{-0} / n_{e 0}=3, \delta_{F}=10, \beta_{F}=0.3$ and $\alpha_{F}=1.263$. The densities are normalized to the initial electron density $n_{e 0}$, and the velocities to the ion acoustic velocity $\sqrt{e T_{e} / m_{+}}$. 
is always true. The positive ion flux being strictly positive (6.4) and the negative ion flux being zero at the wall (boundary condition), the electron flux cannot be zero at the wall (6.11).

What is seen in the results is that for a sufficiently strong magnetic field (high enough value of $\left.\delta_{F}\right)$, the electrons are somewhat confined to the center of the discharge: $n_{e}\left(x_{w}\right)=0$ and $\Gamma_{e}>0$. However, even in that case, the negative ions are still confined: $\Gamma_{-}\left(x_{w}\right)=0$. This means that although the electron density goes to zero at the wall, the electron flux does not while the negative ion flux goes to zero at the wall. The Franklin and Snell model is interesting in that it shows the effect of a strong enough magnetic field on the electron diffusion, but the boundary conditions do not correspond to the situation we want to study: the possibility of extraction of either species of ions. Other boundary conditions are possible, but as described below, a strictly 1D theory does not lead to negative ion extraction.

\subsection{Fluid Model Including End Losses}

The following model has been presented in a paper that has been accepted for publication [72] (see end of the thesis). It uses the fluid equations developed by Franklin and Snell [69], but incorporates end losses into the 1D model, which is necessary to obtain the desired type of solution, in which the flux transverse to a magnetic field consists essentially of positive and negative ions.

\subsubsection{Limitations of a 1D System}

At first, an exhaustive set of processes was considered: ionization, attachment, electronnegative ion detachment, electron-positive ion recombination and ion-ion recombination. However, it was decided to consider a very low pressure case in order to be closer to the experiments. In such a low pressure case, it was assumed that the dominating loss was the positive ion-negative ion recombination, simplifying the problem and allowing a direct study of the magnetic electron filtering.

The equations to be solved are the first and second moments of the fluid equations for electrons, positive ions and negative ions in rectangular geometry

$$
\begin{gathered}
\nabla\left(n_{e} v_{e}\right)=\left(K_{\mathrm{iz}}-K_{\mathrm{att}}\right) n_{g} n_{e} \\
\nabla\left(n_{+} v_{+}\right)=K_{\mathrm{iz}} n_{g} n_{e}-K_{\mathrm{rec}} n_{+} n_{-} \\
\nabla\left(n_{-} v_{-}\right)=K_{\mathrm{att}} n_{g} n_{e}-K_{\mathrm{rec}} n_{+} n_{-} \\
m_{e}\left(K_{\mathrm{iz}} n_{g}+K_{\mathrm{att}} n_{g}+\nu_{e}\right) n_{e} \mathbf{v}_{\mathbf{e}}-e n_{e} \boldsymbol{\nabla} \varphi+e T_{e} \boldsymbol{\nabla} n_{e}+e n_{e} \mathbf{v}_{\mathbf{e}} \times \mathbf{B}_{\mathbf{0}}=0 \\
e T_{+} \nabla n_{+}+e n_{+} \nabla \varphi+m_{+} \nabla\left(n_{+} v_{+} v_{+}\right)+m_{+} \nu_{+} n_{+} v_{+}=0 \\
e T_{-} \nabla n_{-}-e n_{-} \nabla \varphi+m_{-} \nabla\left(n_{-} v_{-} v_{-}\right)+m_{-} \nu_{-} n_{-} v_{-}=0
\end{gathered}
$$

Poisson's equation is replaced by quasi-neutrality

$$
n_{+}=n_{e}+n_{-}
$$

The reaction rates and frequencies are described in section 2.1.1. 
Since quasi-neutrality is assumed, the inertia terms in (6.16) and (6.17), although small over most of the dimension $x$, will stop the simulation when the sound velocity is reached for the ions in case of a sheath.

The initial conditions are $\varphi=0, v_{e,+,-}=0, n_{e}=n_{e 0}, n_{-}=n_{-0}$ and $n_{+}=n_{e 0}+n_{-0}$. The boundary conditions, at some $R$ (one output of the simulation), are chosen to be

$$
\begin{aligned}
& n_{e}(R)=0 \\
& \Gamma_{e}(R)=0 .
\end{aligned}
$$

The boundary condition (6.19) is the same as in the Franklin model. The condition (6.20) is chosen to ensure that the electrons are indeed confined to the center of the discharge. Since this is a one-dimensional fluid model, the relation (6.11) is valid:

$$
\Gamma_{+}=\Gamma_{e}+\Gamma_{-}
$$

Using the boundary conditions (6.19) and (6.20), equations (6.18) and (6.21) give

$$
\begin{aligned}
& n_{+}(R)=n_{-}(R) \\
& \Gamma_{+}(R)=\Gamma_{-}(R),
\end{aligned}
$$

which correspond to obtaining an ion-ion plasma at the edge of the simulation box. It follows that

$$
v_{+}(R)=v_{-}(R)
$$

To integrate the equations, the temperatures of the species are assumed to be constant (the third moment of the fluid equations is not taken into account). Keeping $T_{e}$ constant implies that $\left(K_{\mathrm{iz}}-K_{\mathrm{att}}\right)$ in $(6.12)$ is also constant. Moreover, this term is positive since plasma creation is assumed. Integrating (6.12) over the simulation length and using the initial conditions gives

$$
\Gamma_{e}(R)=\left(K_{\mathrm{iz}}-K_{\mathrm{att}}\right) n_{g} \int_{0}^{R} n_{e}(x) d x .
$$

The boundary condition (6.20) can only be obtained if $K_{\mathrm{iz}}=K_{\mathrm{att}}$ everywhere, in which case there is no electron flux creation. An additional electron loss term, therefore, is needed.

\subsubsection{Electron End Loss}

The previous model considered the cross-field (radial) transport of a one-dimensional electronegative plasma infinite in extent along the magnetic field. However, if the model is to be compared to an actual experiment, the cylinder has a finite length and the fluxes at the ends play a key role in the plasma equilibrium. Since the electrons are the mobile species along the magnetic field lines, the electron flux at the ends cannot be neglected. It should be noted that since the electrons dominate the plasma equilibrium along the magnetic field lines, the sheath is similar to that of an electropositive plasma and the negative ions are confined axially and cannot reach the cylinder ends.

It is possible to represent the electron and positive ion wall losses as volume losses into the one-dimensional model. A two-dimensional cylindrical geometry is thus used to take into account the electron and positive ion end losses for the one-dimensional rectangular 


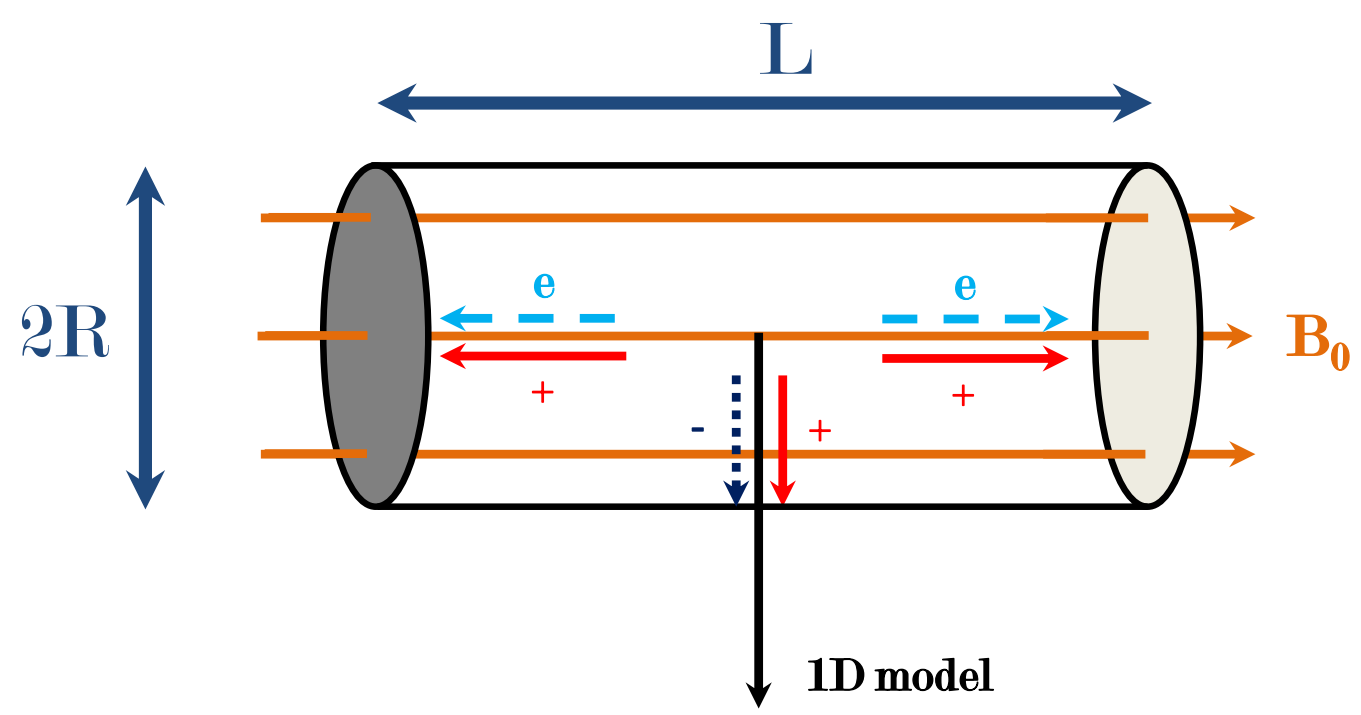

Figure 6.2: Model of the discharge in a finite cylinder of length $L$ and radius $R$. The equations are integrated radially. Electrons (e) are lost axially (at the end walls), positive ions (+) axially and radially, negative ions (-) radially.

fluid model. For this, a finite geometry has to be considered, as shown in figure 6.2. Assuming that the end loss flux $\Gamma_{z}$ and the axial density $n_{+}$are uniform, the wall losses can be written as a volume loss

$$
\nu_{L} n_{+} \approx \frac{2 R}{R L} \Gamma_{z}
$$

with $\nu_{L}$ a loss frequency to be calculated and a factor of 2 because there are two ends. $\Gamma_{z}$ now needs to be calculated. One simple approximate procedure is to use magneticallyconstrained transverse electrons and ambipolar axial flow, giving ion diffusion coefficients $D_{+}$in the transverse directions and $D_{a}$ in the axial direction [7], where

$$
D_{+}=\frac{e T_{+}}{m_{+} \nu_{+}}, \quad D_{a}=D_{+}\left(1+\frac{T_{e}}{T_{+}}\right) .
$$

Choosing rectangular geometry for simplicity, the diffusion equation in a two-dimensional geometry can be written as

$$
-D_{+} \frac{\partial^{2} n_{+}}{\partial x^{2}}-D_{a} \frac{\partial^{2} n_{+}}{\partial z^{2}}=\nu_{i z} n_{e}
$$

Here, the dominating process is assumed to be the volume ionization. This will later be justified by the low density regime in which the wanted solution can be found. For higher densities, the volume recombination of positive and negative ions is worth considering in more detail. Rescaling the z-variable by

$$
z=Z\left(1+\frac{T_{e}}{T_{+}}\right)^{1 / 2}
$$

gives

$$
-D_{+}\left(\frac{\partial^{2} n_{+}}{\partial x^{2}}+\frac{\partial^{2} n_{+}}{\partial Z^{2}}\right)=\nu_{\mathrm{iz}} n_{e}
$$


Making the assumption that $n_{e} \approx n_{e 0}$, Kimura et al [73] have solved (6.30) to find that the diffusion scale lengths in $x$ and $Z$ are approximately the same, and given by the shorter dimension $(R$ or $L)$, such that for $R<\left(T_{e} / T_{+}\right)^{1 / 2} L$,

$$
\frac{d n_{+}}{d Z} \approx-\frac{n_{+}}{R}
$$

Returning to the original coordinate $z$, and using $\Gamma_{z}=-D_{a} \partial n_{+} / \partial z$ yields

$$
\Gamma_{z} \approx D_{+}\left(1+\frac{T_{e}}{T_{+}}\right)^{1 / 2} \frac{n_{+}}{R}
$$

and substituting (6.32) into (6.26) gives

$$
\nu_{L}=\frac{2 D_{+}}{R L}\left(1+\frac{T_{e}}{T_{+}}\right)^{1 / 2} .
$$

The axial electron wall loss is now taken into account as a volume loss.

\subsubsection{Reformulated Model with End Loss}

Adding the electron loss term, equations (6.12) through (6.18) can be rewritten as

$$
\begin{gathered}
\nabla\left(n_{e} v_{e}\right)=\left(K_{\mathrm{iz}}-K_{\mathrm{att}}\right) n_{g} n_{e}-\nu_{L} n_{+} \\
\nabla\left(n_{+} v_{+}\right)=K_{\mathrm{iz}} n_{g} n_{e}-K_{\mathrm{rec}} n_{+} n_{-}-\nu_{L} n_{+} \\
\nabla\left(n_{-} v_{-}\right)=K_{\mathrm{att}} n_{g} n_{e}-K_{\mathrm{rec}} n_{+} n_{-} \\
\mathbf{v}_{\mathbf{e}}\left(K_{\mathrm{iz}} n_{g}+K_{\mathrm{att}} n_{g}+\nu_{e}\right) n_{e} m_{e}-e n_{e} \boldsymbol{\nabla} \varphi+e T_{e} \boldsymbol{\nabla} n_{e}+e n_{e} \mathbf{v}_{\mathbf{e}} \times \mathbf{B}_{\mathbf{0}}=0 \\
e T_{+} \nabla n_{+}+e n_{+} \nabla \varphi+m_{+} \nabla\left(n_{+} v_{+} v_{+}\right)+m_{+} \nu_{+} n_{+} v_{+}=0 \\
e T_{-} \nabla n_{-}-e n_{-} \nabla \varphi+m_{-} \nabla\left(n_{-} v_{-} v_{-}\right)+m_{-} \nu_{-} n_{-} v_{-}=0 \\
n_{+}=n_{e}+n_{-} .
\end{gathered}
$$

All notations are the same as in 6.2.1, except for $\nu_{L}$ from (6.33). A new term $\left(-\nu_{L} n_{+}\right)$ can be seen in (6.34) and (6.35), and corresponds to the electron and positive ion axial loss.

For convenience in obtaining the numerical solutions, the variables are normalized as 
follows:

$$
\left[\begin{array}{rl}
X & =\frac{x}{R_{0}} \\
N_{+, e,-, g} & =\frac{n_{+, e,-, g}}{n_{e 0}} \\
c_{s} & =\left(\frac{e T_{e}}{m_{+}}\right)^{1 / 2} \\
U_{+, e,-} & =\frac{v_{+, e,-}}{c_{s}} \\
\Phi & =-\frac{\varphi}{T_{e}} \\
A_{i, a} & =\frac{n_{e 0} R_{0} K_{\mathrm{iz}, \mathrm{att}}}{c_{s}} \\
B_{i} & =\frac{n_{e 0} R_{0} K_{\mathrm{rec}}}{c_{s}} \\
C_{+, e,-} & =\frac{R_{0} \nu_{+, e,-}}{c_{s}} \\
\Omega & =\frac{R_{0} \omega_{c e}}{c_{s}} \\
\varepsilon_{+,-} & =\frac{T_{e}}{T_{+,-}} \\
\zeta & =\frac{m_{+}}{m_{-}} \\
D & =\frac{R_{0} \nu_{L}}{c_{s}} \\
\Delta_{m} & =\left(A_{i}+A_{a}\right) N_{g}+C_{e} \\
\delta_{m} & =\Delta_{m} \frac{m_{e}}{m_{+}}\left(1+\frac{\Omega^{2}}{\Delta_{m}^{2}}\right) \\
& =
\end{array}\right.
$$

Here, $n_{e 0}$ is the center $x=0$ (on-axis if radial coordinates were used) electron density, $c_{s}$ the usual ion sound velocity (in an electropositive plasma), and $R_{0}$ is a conveniently chosen normalization length without any physical meaning. For easy comparison with experiments, $R_{0}=2.5 \mathrm{~cm}$ is chosen.

With these normalizations, the normalized equations are

$$
\begin{gathered}
\frac{d N_{e} U_{e}}{d X}=\left(A_{i}-A_{a}\right) N_{g} N_{e}-D N_{+} \\
\frac{d N_{+} U_{+}}{d X}=A_{i} N_{g} N_{e}-B_{i} N_{+} N_{-}-D N_{+} \\
\frac{d N_{-} U_{-}}{d X}=A_{a} N_{g} N_{e}-B_{i} N_{+} N_{-} \\
\frac{d N_{e}}{d X}+N_{e} \frac{d \Phi}{d X}+\delta_{m} N_{e} U_{e}=0 \\
\varepsilon_{+}^{-1} \frac{d N_{+}}{d X}-N_{+} \frac{d \Phi}{d X}+\frac{d N_{+} U_{+}^{2}}{d X}+C_{+} N_{+} U_{+}=0 \\
\zeta \varepsilon_{-}^{-1} \frac{d N_{-}}{d X}+\zeta N_{-} \frac{d \Phi}{d X}+\frac{d N_{-} U_{-}^{2}}{d X}+C_{-} N_{-} U_{-}=0
\end{gathered}
$$


Using quasi-neutrality (6.40), $N_{+}=N_{e}+N_{-}$, to eliminate $N_{+}$, gives six equations to determine six variables. With

$$
Y=\left[N_{e} N_{-} U_{+} U_{e} U_{-} \Phi\right]^{T}
$$

the equations can be written in matrix form

$$
M \times Y^{\prime}=R H S
$$

with

$$
M=\left[\begin{array}{cccccc}
U_{e} & 0 & 0 & N_{e} & 0 & 0 \\
U_{+} & U_{+} & N_{+} & 0 & 0 & 0 \\
0 & U_{-} & 0 & 0 & N_{-} & 0 \\
1 & 0 & 0 & 0 & 0 & N_{e} \\
\varepsilon_{i}^{-1}+U_{+}^{2} & \varepsilon_{i}^{-1}+U_{+}^{2} & 2 N_{+} N_{-} & 0 & 0 & -N_{+} \\
0 & \zeta / \varepsilon_{-}+U_{-}^{2} & 0 & 0 & 2 N_{-} U_{-} & \zeta N_{-}
\end{array}\right]
$$

and

$$
R H S=\left[\begin{array}{l}
\left(A_{i}-A_{a}\right) N_{g} N_{e}-D N_{+} \\
A_{i} N_{g} N_{e}-B_{i} N_{+} N_{-}-D N_{+} \\
A_{a} N_{g} N_{e}-B_{i} N_{+} N_{-} \\
-\delta_{m} N_{e} U_{e} \\
-C_{+} N_{+} U_{+} \\
-C_{-} N_{-} U_{-}
\end{array}\right]
$$

The integration is performed in Matlab using

$$
Y^{\prime}=M^{-1} \times R H S
$$

The transverse wall is assumed to be insulating with a potential independent of the grounded ends. The initial conditions are kept identical: $\Phi=0, U_{+, e,-}=0, N_{e}=1$ and $N_{-}=\alpha_{0}$. Here, $\alpha_{0}$ is the central electronegativity $n_{n 0} / n_{e 0}$. The boundary conditions are also kept identical: at some $R$,

$$
\begin{aligned}
& n_{e}(R)=0 \\
& \Gamma_{e}(R)=0
\end{aligned}
$$

At this $R$, the positive and negative ion densities (6.22) and fluxes (6.23) are equal to one another. Because of the thruster context, the ion fluxes have to be positive, which means $v_{+}(R)>0$. In an ion-ion plasma, the upper limit for ion velocities is not the classical Bohm velocity, but an ion Bohm velocity (see section 7.3.2): $u_{B \pm}=\left(e T_{\mp} / m_{ \pm}\right)^{1 / 2}$. Let

$$
\beta=\frac{v_{+}(R)}{u_{B+}} .
$$

In order for the solution to be consistent, the parameter $\beta$ has to fulfill the following condition

$$
0<\beta<1
$$

Beyond $x=R$, there is either an insulating wall or a pure ion-ion plasma. 


\begin{tabular}{ll}
\hline Parameter & Value \\
\hline \hline$K_{\mathrm{iz}}$ & $2.34 \times 10^{-15} T_{e}^{1.03} \exp \left(-12.29 / T_{e}\right)$ \\
$K_{\mathrm{att}}$ & $1.07 \times 10^{-15} T_{e}^{-1.391} \exp \left(-6.26 / T_{e}\right)$ \\
$K_{\mathrm{rec}}$ & $5.2 \times 10^{-14}\left(0.026 / T_{+}\right)^{0.44}$ \\
$\nu_{+}$ & $3.95 \times 10^{-16} n_{g}$ \\
$\nu_{e}$ & $4.7 \times 10^{-14} T_{e}^{1 / 2} n_{g}$ \\
$\nu_{-}$ & $3.95 \times 10^{-16} n_{g}$ \\
\hline
\end{tabular}

Table 6.1: Reaction rates in $m^{3} s^{-1}$ and collision frequencies in $s^{-1}$ for an oxygenlike feedstock gas, except that, for simplicity, the ion collision frequencies are taken to be equal, and the oxygen molecular mass is used for both species.

\subsubsection{Results Using the Reformulated Model}

\section{Numerical Integration}

For convenience, $T_{+}=T_{-}, m_{+}=m_{-}$and a determined ratio $T_{e} / T_{+}$are assumed. These assumptions ensure that the ion-ion plasma obtained at the edge of the simulation is symmetrical, while the constant $T_{e} / T_{+}$ratio simplifies the numerical integration.

It can be seen from (6.33) that $R$ is an input and output of the simulation at the same time. To solve this problem, the fact that the product $R L$ appears in (6.33) is used: the product $R L$ is defined as an input while the variables $R$ and $L$ are outputs. As will be seen in section 6.2.4, curves of constant $R$ and $L$ can be obtained.

The simulations were done with the reaction rates and the collision frequencies of oxygen (see Table 6.1) for comparison with the experiments. The rate coefficients $K_{\mathrm{iz}}$, $K_{\mathrm{att}}, K_{\mathrm{rec}}$ and $K_{\mathrm{m}, \mathrm{e}}\left(\nu_{e}=K_{\mathrm{m}, \mathrm{e}} n_{g}\right)$ are from reference [7], Table 8.2, reactions 1, 2, 4, 7 and 9 , and the rate coefficient $K_{\mathrm{m},+}\left(\nu_{+}=K_{\mathrm{m},+} n_{g}\right)$ from reference [7], p. 366. The pressure is chosen at $p=10$ mTorr, which corresponds to a neutral density $n_{g}=3.3 \times 10^{14} \mathrm{~cm}^{-3}$. A lower limit $n_{e 0} / n_{g}=10^{-7}$ was chosen. The magnetic field is set at $B_{0}=300 \mathrm{G}$, which is an intermediate value where electrons are magnetized and ions only weakly magnetized. The $T_{e} / T_{+}$ratio is set to a nominal value of $T_{e} / T_{+}=20$. The ions are assumed to be $\mathrm{O}_{2}^{+}$ and $\mathrm{O}_{2}^{-}$, with masses $m_{+}=m_{-}=32$ a.m.u. Three values of $R L \in\{100,150,200\} \mathrm{cm}^{-2}$ were studied, with emphasis on $R L=100 \mathrm{~cm}^{-2}$, which is the closest to the thruster cylinder of PEGASES prototype I (see section 3.2.1): $R L=2.3 \times 35.2=81 \mathrm{~cm}^{2}$.

Once the main parameters $\left\{R L, n_{g}, B_{0}, T_{e} / T_{+}, m_{+}\right\}$are chosen, the method for numerical integration is first to choose the initial electron density, $n_{e 0}$, and the initial electronegativity, $\alpha_{0}$. It should noted that $\alpha_{0}$ can be linked to the device dimensions and $n_{e 0}$ to the input power. $T_{e}$ is then the parameter of a shooting method to find both boundary conditions (6.53) and (6.54) fulfilled. When the second boundary condition $\Gamma_{e}(R)=0$ can be fulfilled, depending of the values of the input parameters, it can be so for a relatively wide range of values of $T_{e}$. For each set of input parameters, a minimum value of $T_{e}$ can be calculated to ease the search for that range. The initial source term in the electron balance equation (6.34) has to be positive for the electron flux to be positive,

$$
\left(K_{\mathrm{iz}}-K_{\mathrm{att}}\right) n_{g} n_{e 0}-\nu_{L} n_{+0}>0 .
$$

Since $K_{\mathrm{iz}}, K_{\text {att }}$ and $\nu_{L}$ are known functions of $T_{e}$, this equation can be rewritten as

$$
\frac{K_{\mathrm{iz}}-K_{\mathrm{att}}}{\nu_{L}}>\frac{1+\alpha_{0}}{n_{g}},
$$




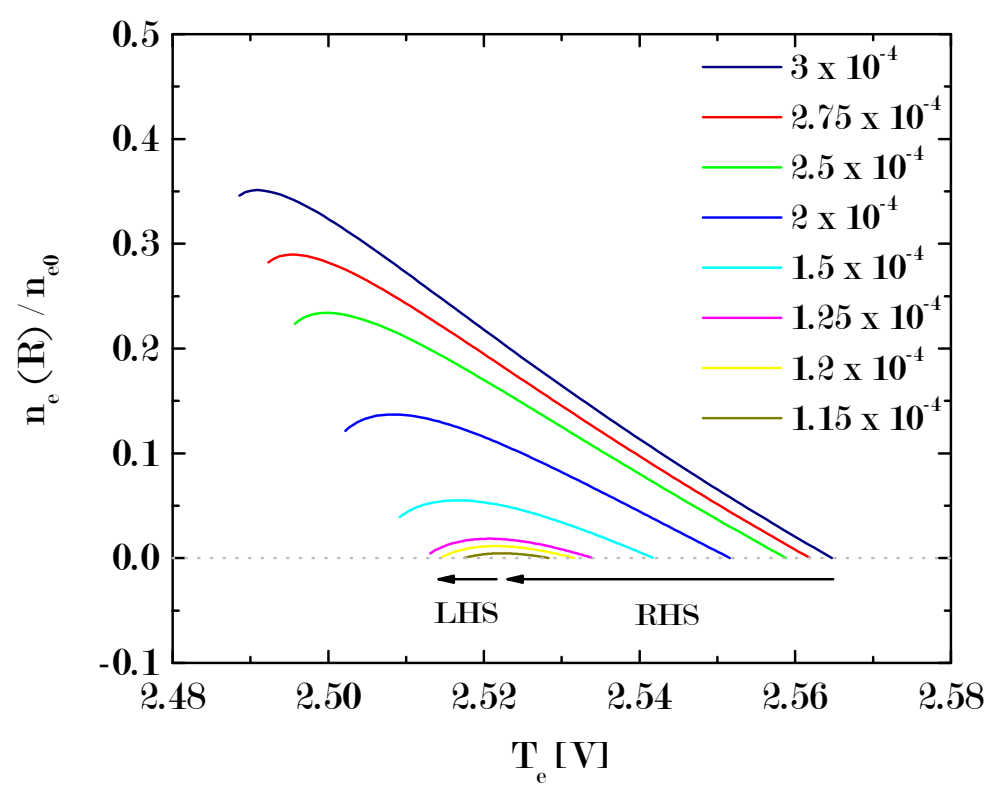

Figure 6.3: $n_{e}(R) / n_{e 0}$ with the boundary condition $\Gamma_{e}(R)=0$ fulfilled as a function of $T_{e}$ for $R L=100 \mathrm{~cm}^{2}, \alpha_{0}=1.0$ and $n_{e 0} / n_{g}$ from $3 \times 10^{-4}$ (top curve) to $1.15 \times 10^{-4}$ (bottom curve). The right hand side (RHS) and left hand side (LHS) solutions are shown as arrows indicating increasing $\beta$.

determining the minimum value of $T_{e}$. With this range of $T_{e}$, two situations can arise, either there is only one $T_{e}$ fulfilling the first boundary condition $n_{e}(R)=0$ as well, or there are two $T_{e}$ 's fulfilling $n_{e}(R)=0$. For $R L=100 \mathrm{~cm}^{2}$ and $\alpha_{0}=1.0$, figure 6.3 shows $n_{e}(R) / n_{e 0}$ when $\Gamma_{e}(R)=0$ is fulfilled as a function of $T_{e}$ for decreasing $n_{e 0} / n_{g}$ from $3 \times 10^{-4}$ to $1.15 \times 10^{-4}$. For $3 \times 10^{-4} \geq n_{e 0} / n_{g} \geq 1.25 \times 10^{-4}$, there is only one $T_{e}$ fulfilling the two boundary conditions, and this $T_{e}$ is the maximum value of the $T_{e}$ range (right hand side of the curve). From $n_{e 0} / n_{g}=1.2 \times 10^{-4}$ and decreasing, there are two $T_{e}$ 's fulfilling both boundary conditions: the minimum and maximum values of the $T_{e}$ range (both extremities of the curve). It can be seen from the evolution of the $n_{e 0} / n_{g}$ curves that for the minimum value of $n_{e 0} / n_{g}$ giving both boundary conditions fulfilled, both $T_{e}$ solutions become the same solution. In the region to the left of the curves (lower $T_{e}$ ), both $n_{e}(R)$ and $\Gamma_{e}(R)$ are finite and positive (see section 6.4.3).

The two main output parameters are $R$, the simulation length, and $\beta=v_{+}(R) / u_{B+}$. Only simulations fulfilling (6.56) are considered to be solutions. $\beta=0$ corresponds to a volume balance of attachment and recombination, while $\beta=1$ corresponds to the ion sound limit where quasi-neutrality is violated. Because of the inertia terms in (6.35) and (6.36), no solution fulfilling the boundary conditions can be found with $\beta>1$.

Figure 6.4 is complementary to figure 6.3 in that it shows the $\beta$ 's corresponding to the $n_{e}(R)$ curves: $R L=100 \mathrm{~cm}^{2}, \alpha_{0}=1.0$ and $3 \times 10^{-4} \geq n_{e 0} / n_{g} \geq 1.15 \times 10^{-4}$. The right hand side solutions correspond to $\beta$ from 0 to an intermediate value around 0.3 while the left hand side solutions correspond to $\beta$ from this intermediate value to 1 . The intermediate value of $\beta=0.3$ is specific to $\alpha_{0}=1.0$. 


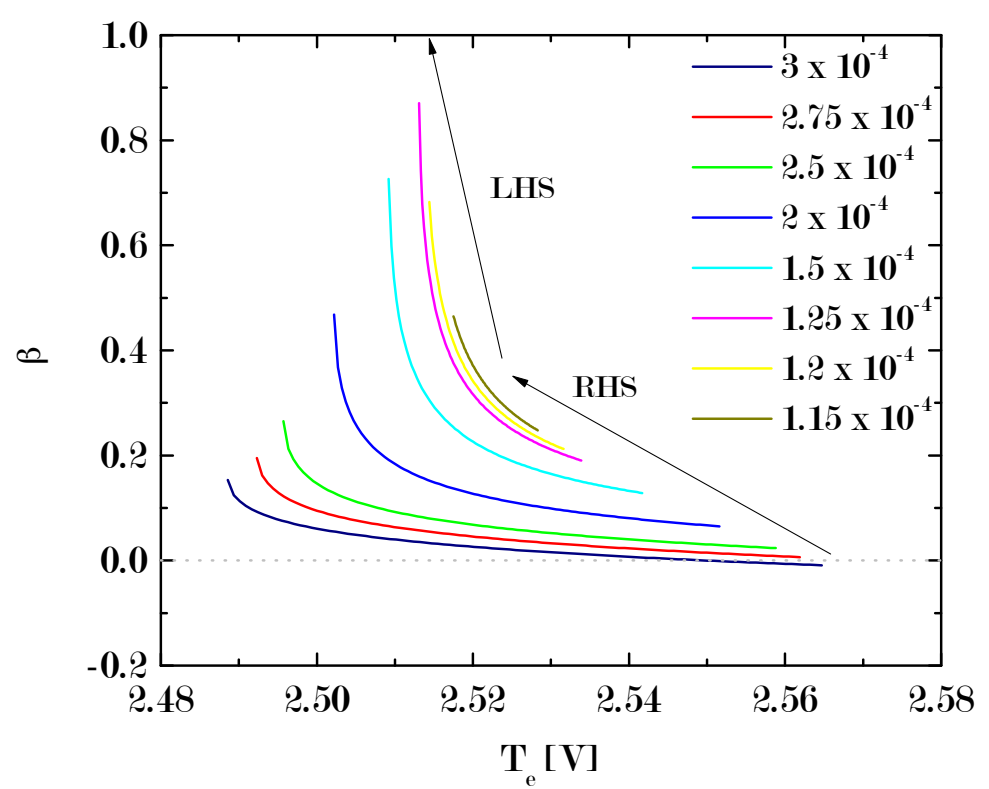

Figure 6.4: $\beta$ with the boundary condition $\Gamma_{e}(R)=0$ fulfilled as a function of $T_{e}$ for $R L=100 \mathrm{~cm}^{2}, \alpha_{0}=1.0$ and $n_{e 0} / n_{g}$ from $3 \times 10^{-4}$ (bottom curve) to $1.15 \times 10^{-4}$ (top curve). The right hand side (RHS) and left hand side (LHS) solutions are shown as arrows indicating increasing $\beta$.

\section{Simulation Results}

Solving as described above for a fixed $n_{e 0} / n_{g}=2.75 \times 10^{-4}$, two values of $\alpha_{0}$ show two characteristic behaviors in figure 6.5 and 6.6. Figure 6.5 shows the density (a), potential (b) and flux profiles (c) for $\alpha=1.0$, close to the limit value of $\beta=0$. Figure 6.6 shows the same profiles for $\alpha=0.828$, close to the limit value of $\beta=1$. In both figures, the electron density and flux go to zero at the radial boundary $x=R$, satisfying the boundary conditions (6.53) and (6.54). As a result, the positive ion density equals the negative ion density at $x=R$, which was predicted in (6.22). Similarly, the ion fluxes become equal at $x=R$, predicted in (6.23). The profile of the potential indicates that the electric field vanishes at $x=R$, confirming that there is no sheath and either species of ions may be extracted. Finally, it should be noted that the electron density profile is unchanged with a form similar to $n_{e 0}\left(1+\cos \frac{\pi x}{R}\right)$.

In the $\beta \approx 0$ case (figure 6.5 ) the negative ion density is relatively constant while the ion fluxes pass through a maximum at about half the simulation length. The evolution of the negative ion flux shows how the decrease of electron density influences the attachment and recombination terms. In the first half of the simulation, $K_{\text {att }} n_{g} n_{e}>K_{\text {rec }} n_{+} n_{-}$, but in the second half, $K_{\text {att }} n_{g} n_{e}<K_{\text {rec }} n_{+} n_{-}$. As stated previously, the $\beta=0$ case corresponds to a volume balance of attachment and recombination since the integrated values of the two terms cancel each other. In the $\beta \approx 1$ case (figure 6.6), the ion densities decay towards the edge while the ion fluxes increase, reaching a plateau near the edge. The negative ion flux shows that in this situation, $K_{\text {att }} n_{g} n_{e}>K_{\text {rec }} n_{+} n_{-}$holds true for the entire simulation length, with $K_{\text {att }} n_{g} n_{e}-K_{\text {rec }} n_{+} n_{-} \approx 0$ as all densities decay. This solution seems to be the desired one for the PEGASES thruster concept, with a maximum velocity for ions to be extracted. However, it will be shown that $\beta=1$ does not result in the maximum flux. 


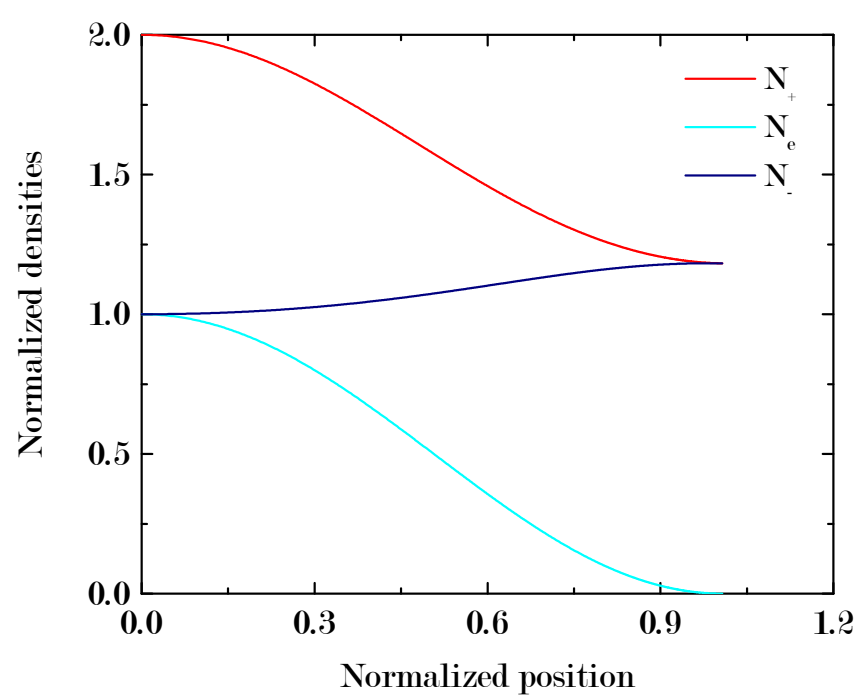

(a)

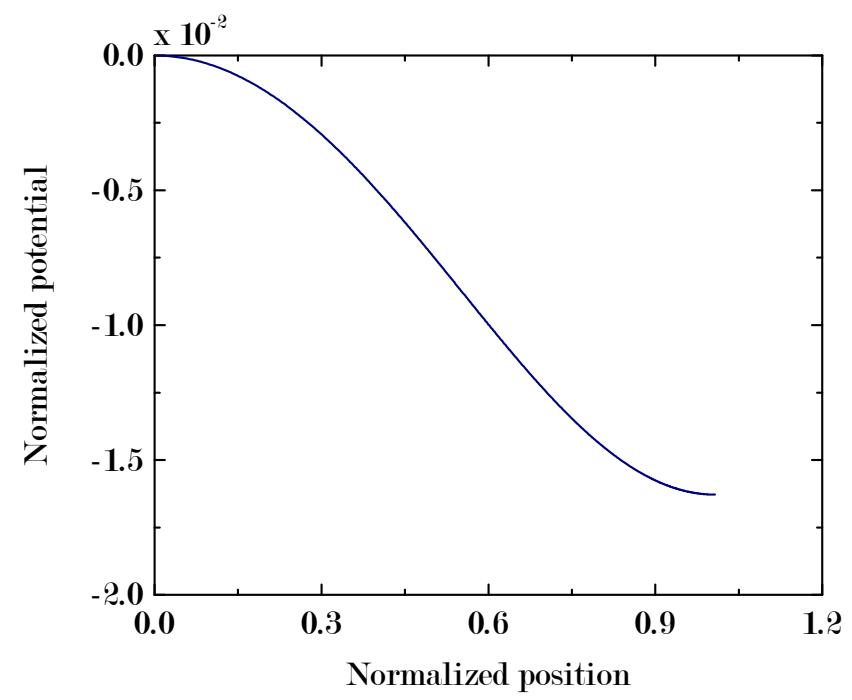

(b)

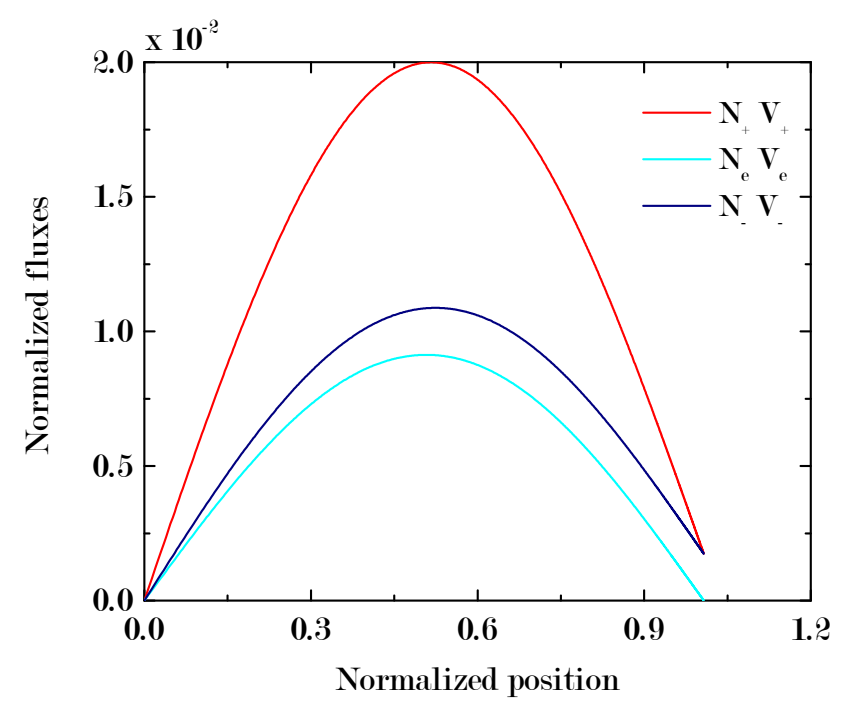

(c)

Figure 6.5: Normalized densities (a), potential (b) and fluxes (c) as functions of normalized position for $\beta \approx 0$ with $\alpha_{0}=1.0$ and $n_{e 0} / n_{g}=2.75 \times 10^{-4}$. Normalizations are $X=x / R_{0} ; N_{+}, N_{e}, N_{-}=n_{+, e,-} / n_{e 0} ; U_{+, e,-}=v_{+, e,-} /\left(n_{e 0} c_{s}\right) ; \Phi=-\varphi / T_{e}$. 


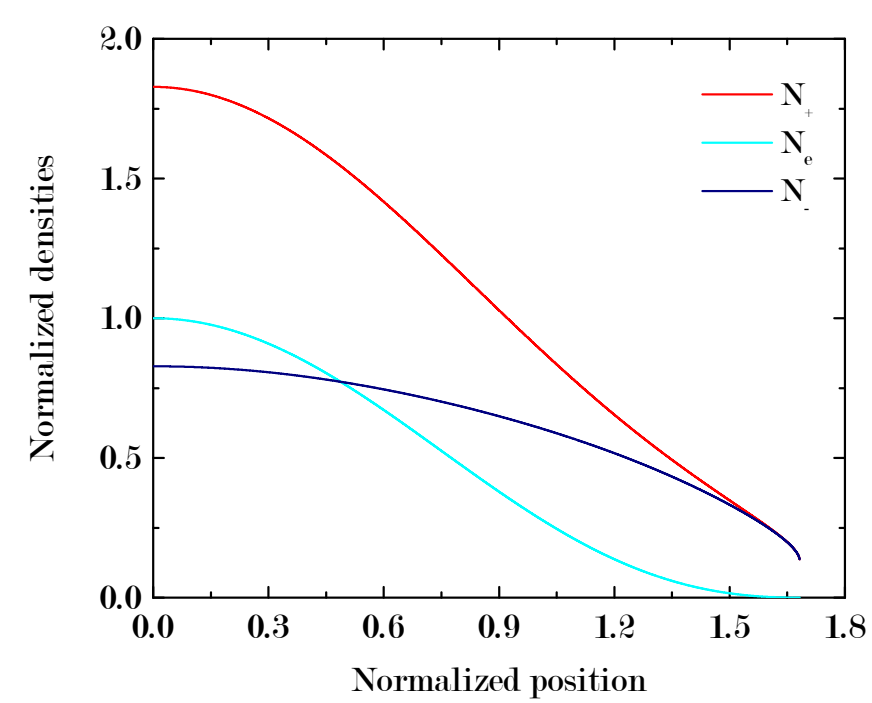

(a)

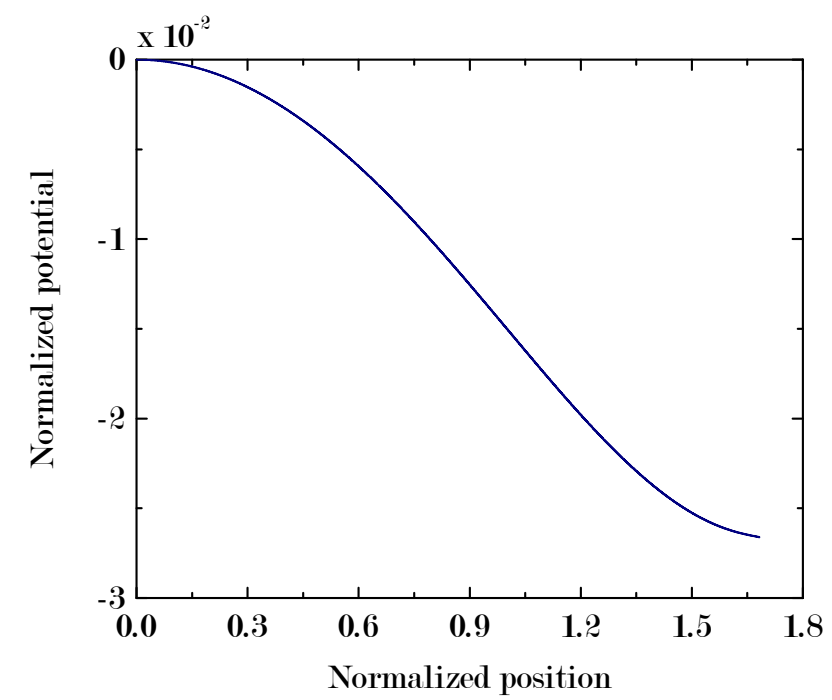

(b)

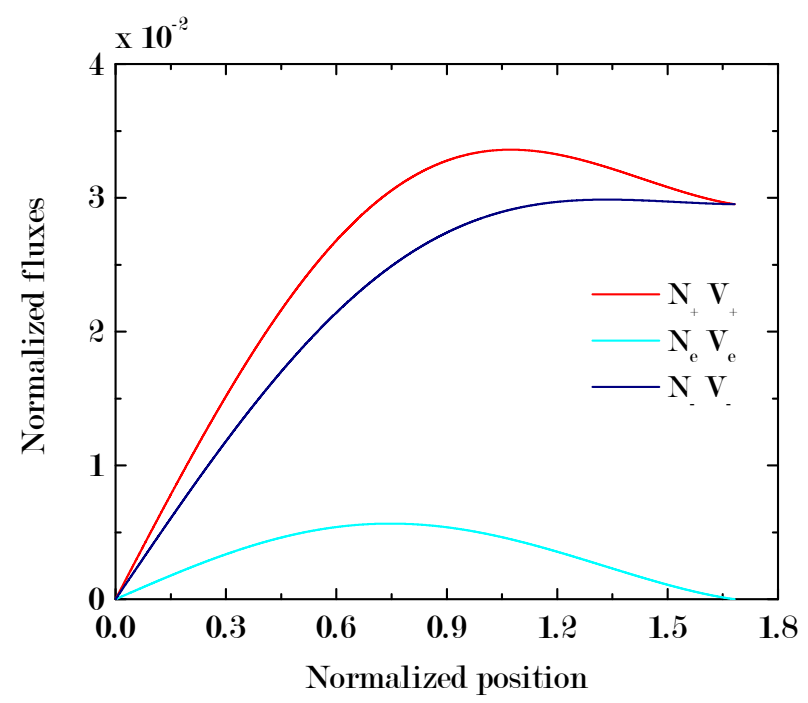

(c)

Figure 6.6: Normalized densities (a), potential (b) and fluxes (c) as functions of normalized position for $\beta \approx 1$ with $\alpha_{0}=0.828$ and $n_{e 0} / n_{g}=2.75 \times 10^{-4}$. Normalizations are $X=x / R_{0} ; N_{+}, N_{e}, N_{-}=n_{+, e,-} / n_{e 0} ; U_{+, e,-}=v_{+, e,-} /\left(n_{e 0} c_{s}\right) ; \Phi=-\varphi / T_{e}$. 


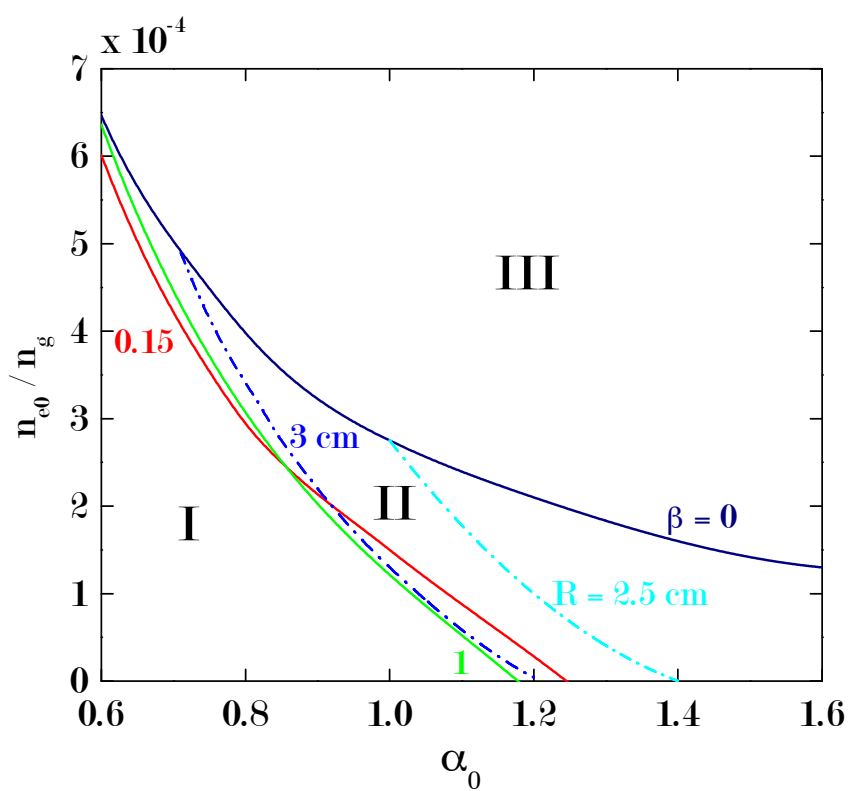

Figure 6.7: $n_{e 0} / n_{g}$ versus $\alpha_{0}$ for $p=10 \mathrm{mTorr}, B_{0}=300 \mathrm{G}, R L=100 \mathrm{~cm}^{-2}$, $T_{e} / T_{i}=20$ and $M_{i}=32$ a.m.u. The upper-through-lower solid curves are for $\beta=0$, 0.15 and 1 , respectively; the dash-dot curves give $R=3 \mathrm{~cm}$ and $R=2.5 \mathrm{~cm}$, which are the solutions for two fixed device configurations.

\section{The $\left(\alpha_{0}, n_{e 0} / n_{g}\right)$ Plane}

In figure 6.7, the numerical solutions are shown for $n_{e 0} / n_{g}$ versus $\alpha_{0}$, with $\beta$ as a parameter, for $p=10 \mathrm{~m}$ Torr and $R L=100 \mathrm{~cm}^{-2}$. Contours of constant $\beta$ for $0,0.15$ and 1 are shown. Curves of constant $R=3 \mathrm{~cm}(L=33.3 \mathrm{~cm})$ and constant $R=2.5 \mathrm{~cm}(L=40 \mathrm{~cm})$ are shown as dash-dot lines.

To construct the $\beta$-curves, a fixed value of $n_{e 0}\left(\right.$ or $\left.\alpha_{0}\right)$ is first chosen. Next, a sequence of values of $\alpha_{0}$ (or $n_{e 0}$ ) is chosen, corresponding to a horizontal (or vertical) scan in the figure. Finally, for each value of $\alpha_{0}$ and $n_{e 0}$ along this horizontal (or vertical) line, a set of $T_{e}$ values are investigated. For each $T_{e}$, the equations are integrated to the point where $\Gamma_{e}(R) \approx 0$. If a value of $T_{e}$ also makes $n_{e}(R) \approx 0$, then a solution has been found and the value of $\beta$ is calculated. It should be noted that $\beta=1$ does not correspond to $U_{+}=1$ but to $U_{+}=\left(T_{e} / T_{+}\right)^{-1 / 2}=20^{-1 / 2}$ since the normalization was done to $c_{s}=\left(e T_{e} / m_{+}\right)^{1 / 2}$ and the ion Bohm velocity is $\left(e T_{+,-} / m_{-,+}\right)^{1 / 2}$. The approximate bounding values of $\beta=0$ and $\beta=1$ are shown as solid lines. The $\beta=1$ cannot be obtained exactly due to the inertia-caused singularity, and therefore, is represented by points with $\beta$ close to 1 .

This figure shows that for a given electronegativity $\alpha_{0}$, there is a specific range of ionization fraction values $n_{e 0} / n_{g}$ that yields the wanted solutions. The region to the left of the $\beta \approx 1$ curve (noted I) is a region where $\beta$ would need to be greater than 1 in order to find the boundary conditions fulfilled: the desired solution cannot be found. The region to the right of the $\beta \approx 0$ curve (noted III) corresponds to negative $\beta$ 's, meaning that an input of ion fluxes is needed to sustain the plasma. These solutions do not match the need of a thruster. Between those two regions is a central region (noted II) where all the self-sustained solutions fulfilling the boundary conditions can be found. This is the region of interest to extract ions.

Numerically, it was found that the $\beta$-curves can intersect for different values of $\beta$. 
For example, the $\beta \approx 0.15$ curve intersects the $\beta \approx 1$ curve at $\alpha_{0}=0.86$ and $n_{e 0} / n_{g}=$ $2.4 \times 10^{-4}$. The $T_{e}$ 's for these solutions at the intersection are, of course, different; i.e., $T_{e} \approx 2.49 \mathrm{~V}$ at $\beta \approx 1$ is lower than $T_{e} \approx 2.52 \mathrm{~V}$ at $\beta \approx 0.15$. The $T_{e}$ 's being different, the solutions are indeed different in the sense that the $R$ values (and corresponding $L$ ) are different, describing two distinct situations. As was described in 6.2.4, the intersection of two $\beta$-curves correspond to the situation where there are two $T_{e}$ 's fulfilling both boundary conditions. With decreasing $n_{e 0} / n_{g}$ and increasing $\alpha_{0}$, the intersection occurs between $\beta=1$ and increasing values of $\beta$ : $\beta=0.15$ for $\alpha_{0}=0.86$ and $n_{e 0} / n_{g}=2.4 \times 10^{-4}$, and $\beta=0.2$ for $\alpha=1.0$ and $n_{e 0} / n_{g}=1.2 \times 10^{-4}$. It should be noted that there is only one value of $T_{e}$ giving the wanted solution on the $\beta=0$-curve in the $\left(\alpha_{0}, n_{e 0} / n_{g}\right)$ space shown.

The construction of the $R$-curves is the same as for the $\beta$-curves. Of course, $\beta$ is not constant over an $R$-curve, as it can be seen on the figure since, for instance, the $R=3 \mathrm{~cm}$ curve intersects with all three curves of constant $\beta(0,0.15$ and 1$)$. As a result, for a given configuration $(R$ and $L)$, there is a range of parameters $\alpha_{0}$ and $n_{e 0} / n_{g}$ giving possible values of $\beta$ between 0 and a maximum value. It can be seen on figure 6.7 that the maximum value for $R=3 \mathrm{~cm}$ is bigger than that for $R=2.5 \mathrm{~cm}$. In the cases where there are two $T_{e}$ 's giving a solution for a single $\left(\alpha_{0}, n_{e 0} / n_{g}\right), R$ constant is accompanied by a continuous evolution of $T_{e}$. The second $T_{e}$ solution (and therefore $R$ ) is also continuous, but for different values.

For constant $n_{e 0} / n_{g}$, increasing $\alpha_{0}$ results in an increase of $T_{e}$. This can be explained by looking at the integrated electron flux conservation (6.34) and assuming that the negative ion density is constant

$$
0=\left[\left(K_{\mathrm{iz}}-K_{\mathrm{att}}\right) n_{g}-\left(1+\alpha_{0}\right) \nu_{L}\right] \int_{0}^{R} n_{e}(x) d x .
$$

Since the integral term is constant, an increase of $\alpha_{0}$ has to be compensated by an increase of $\left(K_{\mathrm{iz}}-K_{\mathrm{att}}\right)$, which corresponds to an increase of $T_{e}$. The fact that $\nu_{L}$ is also an increasing function of $T_{e}$ does not play a significant role because of the different increasing rates. With an increase in $T_{e}$, the electron source and loss term are bigger, meaning a faster increase then decrease of the electron flux: for an increasing $\alpha_{0}, R$ decreases. For constant $\alpha_{0}$, increasing $n_{e 0} / n_{g}$ also results in an increase of $T_{e}$ and a decrease of $R$. However, (6.59), with the constant negative ion density assumption, would lead to the conclusion that $T_{e}$ is independent of $n_{e 0} / n_{g}$, as will be derived later in the analytic model in (6.73) in section 6.3.1.

\section{The $R L$ parameter}

Figure 6.8 shows the $\beta=0$ and $\beta=0.15$ curves in the $\left(\alpha_{0}, n_{e 0} / n_{g}\right)$ plane for the three values of $R L \in\{100,150,200\} \mathrm{cm}^{2}$. As it was seen on figure 6.7 , the $\beta=0.15$ and $\beta=1$ curves are extremely close to one another, allowing the use of the much easier obtained $\beta=0.15$ curve to delimit the space of wanted solutions (in-between the $\beta=0$ and $\beta=1$ curves). The evolution of this space, therefore, can be seen with $R L$ as a parameter. Changing the value of $R L$ changes the value of $\nu_{L}$ (6.33), which scales as $\nu_{L} \propto(R L)^{-1}$. The first thing to note is that the $\beta=0$ curve does not change with $R L$. Since $\beta=0$ is a volume balance between attachment and recombination in the negative ion flux conservation equation (6.36), and that the electron end loss term only plays a role in the electron and positive ion flux conservations, $\beta=0$ does indeed not depend on $\nu_{L}$. 


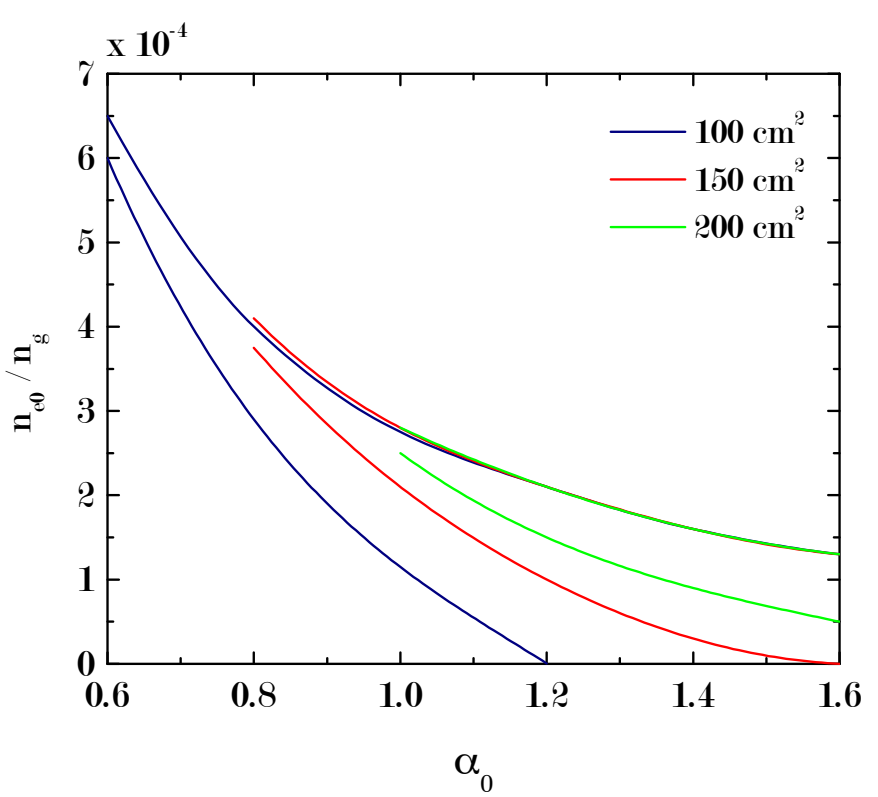

Figure 6.8: $n_{e 0} / n_{g}$ versus $\alpha_{0}$ for $p=10$ mTorr, $B_{0}=300 G$ and $T_{e} / T_{i}=20$. For each value of $R L$ (100, 150 and $\left.200 \mathrm{~cm}^{2}\right)$, the $\beta=0$ and $\beta=0.15$ are shown, delimiting the space of wanted solutions.

\begin{tabular}{c|ccc}
\hline$R L\left(\mathrm{~cm}^{2}\right)$ & 100 & 150 & 200 \\
\hline \hline$T_{e}(\mathrm{~V})$ & 2.60 & 2.49 & 2.43 \\
$R(\mathrm{~cm})$ & 2.30 & 2.85 & 3.33 \\
\hline
\end{tabular}

Table 6.2: $T_{e}$ and $R$ as a function of $R L$ for a given point on the $\beta=0$ curve in the $\left(\alpha_{0}, n_{e 0} / n_{g}\right)$ plane: $\alpha_{0}=1.2$ and $n_{e 0} / n_{g}=2.1 \times 10^{-4}$.

For chosen $\alpha_{0}=1.2$ and $n_{e 0} / n_{g}=2.1 \times 10^{-4}$ (on the $\beta=0$ curve), table 6.2 shows the evolution of $R$ and $T_{e}$ as a function of $R L$. The way $R$ and $T_{e}$ scale can be explained by looking at the integrated electron flux conservation (6.34) with the following assumptions: constant negative ion density and $n_{e}(x)=\frac{n_{e 0}}{2}\left(1+\cos \frac{\pi x}{R}\right)$, then divided by $n_{e 0}$,

$$
0=\left(K_{\mathrm{iz}}-K_{\mathrm{att}}\right) n_{g}-\nu_{L}\left(1+\alpha_{0}\right) .
$$

With a reasoning similar to the evolution of $T_{e}$ and $R$ as functions of $\alpha_{0}$ and $n_{e 0} / n_{g}$, an increase in $R L$ results in a decrease of $T_{e}$ and an increase of $R$.

The evolution of the $\beta=0.15$ curve in figure 6.8 comes from the evolution of $T_{e}$ and $R$. Moving downward from the $\beta=0$ curve corresponds to increasing $\beta$ 's. With increasing $R L, R$ also increases, which means that for a given ratio between volume attachment and recombination, $\beta$ is increasing with $R L$. Therefore, the limiting value of $\beta=1$ is reached closer to the $\beta=0$ curve as $R L$ is increased. As the $\beta=0$ and $\beta=1$ curves get closer, simulation results become harder to obtain, which is why the $\beta$ curves begin at $\alpha_{0}=0.8$ for $R L=150 \mathrm{~cm}^{2}$ and $\alpha_{0}=1.0$ for $R L=200 \mathrm{~cm}^{2}$.

\section{The $B_{0}$ parameter}

In the model, the magnetic field only appears in the second moment of the fluid equations for the electrons. For small values of the magnetic field $\left(B_{0}\right)$, the model is consistent, but 


\begin{tabular}{c|ccccc}
\hline$B_{0}(\mathrm{G})$ & 200 & 300 & 400 & 500 & 600 \\
\hline \hline$T_{e}(\mathrm{~V})$ & 2.56 & 2.60 & 2.60 & 2.61 & 2.61 \\
$R(\mathrm{~cm})$ & 4.23 & 2.30 & 1.66 & 1.32 & 1.10 \\
\hline
\end{tabular}

Table 6.3: $T_{e}$ and $R$ as functions of $B_{0}$ for $p=10 \mathrm{mTorr}, R L=100 \mathrm{~cm}^{2}$, $T_{e} / T_{i}=20, \alpha_{0}=1.2$ and $n_{e 0} / n_{g}=2.1 \times 10^{-4}$.

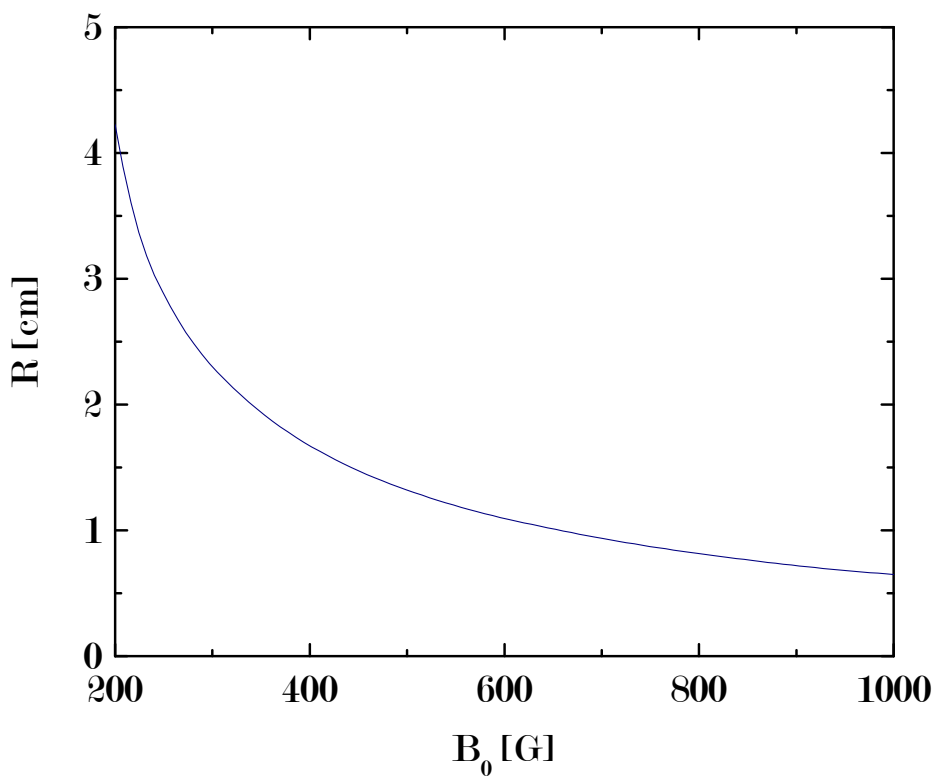

Figure 6.9: $R$ as a function of $B_{0}$ for $p=10 \mathrm{mTorr}, R L=100 \mathrm{~cm}^{2}, T_{e} / T_{i}=20$, $\alpha_{0}=1.2$ and $n_{e 0} / n_{g}=2.1 \times 10^{-4}$.

does not correspond to the desired regime of magnetically confined electrons. For large values of the magnetic field, the model is no longer valid since the ions are assumed to be unmagnetized (no magnetic force in the momentum conservation equations for ions).

Table 6.3 shows the evolution of $T_{e}, R$ and $\beta$ for values of $B_{0}$ between $200 \mathrm{G}$ and $600 \mathrm{G}$ with $p=10$ mTorr, $R L=100 \mathrm{~cm}^{2}, T_{e} / T_{i}=20, \alpha_{0}=1.2$ and $n_{e 0} / n_{g}=2.1 \times 10^{-4}$. For these parameters, the value of $B_{0}=100 \mathrm{G}$ cannot be computed, causing the model to break down. $T_{e}$ can be seen to be almost insensitive to the magnetic field (small increase), while $R$ is a decreasing function of the magnetic field. With a magnetic field increase, the electrons are more confined to the center as their diffusion is further impeded. As to $\beta$, it is almost insensitive to the magnetic field (small decrease). For more clarity, figure 6.9 shows the evolution of $R$ as a function of the magnetic field $B_{0}$.

\subsection{Analytic Approximation}

\subsubsection{Analytic Model}

The equations of the complete model are integrated with a shooting method on the parameter $T_{e}$. For one set of input parameters, there might be one, two or no $T_{e}$ giving the wanted solutions. Therefore, it is costly to search the $\left(\alpha_{0}, n_{e 0} / n_{g}\right)$ space for solutions. In order to understand the scaling of magnetic electron filtering in a much quicker way, an 
analytic model was developed, using the observations of the numerical results.

The same geometry is considered: a finite cylinder approximated in 2D by a length $L$ and a half-width $R$ in which electrons are lost axially, the positive ions axially and radially, and the negative ions radially. The axial losses are approximated as volume losses in a one-dimensional fluid model (see section 6.2.3). The parameters $B_{0}, R L$ and $T_{e} / T_{i}$ are input parameters, as in the numerics. For simplicity, the non-linear inertia terms in the ion equations (6.38) and (6.39) are dropped. Neglecting the ionization and attachment collision terms, the second moment fluid equation for the electrons can be written as

$$
\Gamma_{e}=-D_{\perp e} \frac{d n_{e}}{d x}-\mu_{\perp e} n_{e} E
$$

with

$$
\begin{aligned}
D_{\perp e} & =\frac{D_{e}}{1+\omega_{c e}^{2} / \nu_{e}^{2}}, & D_{e} & =\frac{e T_{e}}{m_{e} \nu_{e}}, \\
\mu_{\perp e} & =\frac{\mu_{e}}{1+\omega_{c e}^{2} / \nu_{e}^{2}}, & \mu_{e} & =\frac{e}{m_{e} \nu_{e}} .
\end{aligned}
$$

In order to compare the two terms in (6.61) (diffusion and mobility), they are approximated. The magnetic field is assumed to be large enough that the radial electric field scales with the ion temperature, $E \sim T_{+} / R$, while the electron density gradient is approximated with $d n_{e} / d x \sim-n_{e 0} / R$ (linear approximation between the initial condition $n_{e}(0)=n_{e 0}$ and the boundary condition $\left.n_{e 0}(R)=0\right)$. The assumption on the electron density profile is only used in this comparison. These assumptions lead to the following scaling for the two terms of (6.61)

$$
\begin{aligned}
D_{\perp e} \frac{d n_{e}}{d x} & \sim \frac{e / m_{e} \nu_{e}}{1+\omega_{c e}^{2} / \nu_{e}^{2}} \frac{n_{e 0}}{R} T_{e} \\
\mu_{\perp e} n_{e} E & \sim \frac{e / m_{e} \nu_{e}}{1+\omega_{c e}^{2} / \nu_{e}^{2}} \frac{n_{e 0}}{R} T_{+} .
\end{aligned}
$$

Since $T_{+} \ll T_{e}$, the drift term (6.65) is small compared to the diffusion term (6.64), simplifying (6.61) to

$$
\Gamma_{e} \approx-D_{\perp e} \frac{d n_{e}}{d x} .
$$

The electron axial losses are integrated as in the numerics, keeping in mind that the electron density can be assumed constant over the axial direction $z$ for $L \gg R$. Combining (6.66) with the electron first moment fluid equation yields

$$
-D_{\perp e} \frac{d^{2} n_{e}}{d x^{2}}=\left(\nu_{\mathrm{iz}}-\nu_{\mathrm{att}}\right) n_{e}-\nu_{L}\left(n_{e}+n_{-}\right)
$$

with quasi-neutrality $n_{+}=n_{e}+n_{-}$. Here, $\nu_{\text {iz }}=K_{\text {iz }} n_{g}$ is the ionization frequency, $\nu_{\text {att }}=$ $K_{\text {att }} n_{g}$ the attachment frequency, and $\nu_{L}$ the electron end loss term (6.33) which is a volume loss frequency corresponding to the electron and positive ion axial losses. The sign of the right hand side of (6.67) plays an important role and depends mainly on the electron density, as was discussed in the numerics. This equation can be rewritten as

$$
\frac{d^{2} n_{e}}{d x^{2}}+\frac{\nu_{\mathrm{net}}}{D_{\perp e}} n_{e}=\frac{\nu_{L}}{D_{\perp e}} n_{-},
$$




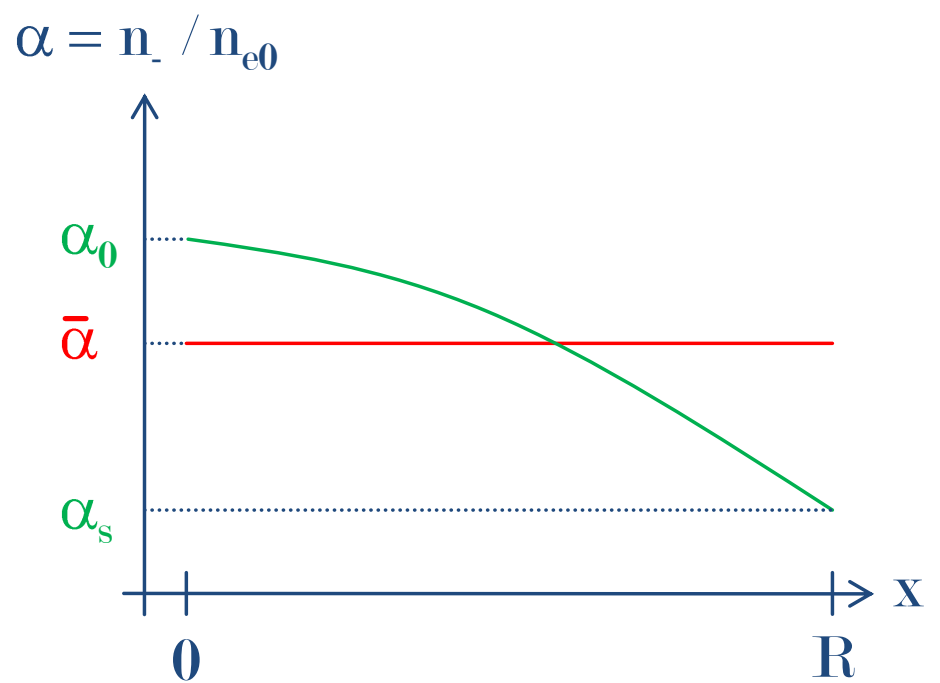

Figure 6.10: Electronegativities $\alpha=n_{-} / n_{e 0}$ used in the analytic approximation: $\alpha_{0}$ initial electronegativity, $\alpha_{s}$ electronegativity at $x=R, \bar{\alpha}$ average electronegativity.

with $\nu_{\text {net }}=\nu_{\text {iz }}-\nu_{\text {att }}-\nu_{L}$.

Assuming that the negative ion density is constant $\left(n_{-}=\bar{n}_{-}\right)$, as was approximately obtained in the simulation results, renders (6.68) easy to solve:

$$
n_{e}(x)=A \cos (\omega x+B)+\frac{\nu_{L}}{\nu_{\text {net }}} \bar{n}_{-},
$$

with $A$ and $B$ two constants to be determined and $\omega^{2}=\frac{\nu_{\text {net }}}{D_{\perp}}$. The boundary conditions come from the boundary conditions in the numerics and the observations of the simulation results:

$$
\left.\frac{d n_{e}}{d x}\right|_{x=0}=0,\left.\quad \frac{d n_{e}}{d x}\right|_{x=R}=0, \quad n_{e}(0)=n_{e 0}, \quad n_{e}(R)=0 .
$$

This set of boundary conditions is satisfied by

$$
n_{e}(x)=\frac{n_{e 0}}{2}\left(1+\cos \frac{\pi x}{R}\right) .
$$

By identification between (6.69) and (6.71), the following relations are obtained;

$$
\frac{\nu_{\text {net }}}{D_{\perp e}}=\frac{\pi^{2}}{R^{2}}, \quad A=\frac{\nu_{L}}{\nu_{\text {net }}} \bar{n}_{-}=\frac{n_{e 0}}{2}, \quad B=0
$$

Let $\bar{\alpha}=\bar{n}_{-} / n_{e 0}$ be the average electronegativity. The edge electronegativity at $x=R$ is noted $\alpha_{s}$. It should be noted that the electron density is taken as the initial electron density $n_{e 0}$ in the expression of the electronegativity in this chapter. In the case of a decreasing electronegativity, figure 6.10 summarizes the electronegativities $\alpha, \alpha_{0}, \alpha_{s}$ and $\bar{\alpha}$. Rewriting the expression of the constant in (6.72) yields $\bar{\alpha}=\frac{\nu_{\text {net }}}{2 \nu_{L}}$, which is equivalent to $(1+2 \bar{\alpha}) \nu_{L}=\nu_{\mathrm{iz}}-\nu_{\text {att }}$. Replacing $\nu_{L}$ with its expression from (6.33) gives

$$
\frac{2 D_{+}}{R L}(1+2 \bar{\alpha})\left(1+\frac{T_{e}}{T_{+}}\right)^{1 / 2}=\left(K_{\mathrm{iz}}-K_{\mathrm{att}}\right) n_{g} .
$$


As in the numerics, $R L$ is set for a given problem in order to simplify the resolution of the equations. By using $\bar{\alpha}$ instead of $\alpha_{0}$ (numerics) as an input parameter, and keeping $T_{e} / T_{+}$constant, (6.73) is the expression of the function $T_{e}(\bar{\alpha})$. It should be noted that $T_{e}$ is a function of $\bar{\alpha}$ without dependence on $n_{e 0} / n_{g}$. Combining $\bar{\alpha}=\nu_{\text {net }} /\left(2 \nu_{L}\right)$ and $\nu_{\text {net }} / D_{\perp e}=\pi^{2} / R^{2}$, and using the expression of $\nu_{L}$ gives $R$ as a function of the parameters $R L, \bar{\alpha}$ and $T_{e}$ :

$$
R^{2}=\frac{\pi^{2}}{4 \bar{\alpha}} \frac{D_{\perp e}}{D_{+}} \frac{R L}{\left(1+T_{e} / T_{+}\right)^{1 / 2}} .
$$

From $R$, the value $L$ can, of course, be calculated. For sufficiently high magnetic fields $\left(\omega_{\mathrm{ce}} / \nu_{e} \gg 1\right), D_{\perp e}$ can be approximated with $\left(e T_{e} \nu_{e}\right) /\left(m_{e} \omega_{\mathrm{ce}}^{2}\right)$. This leads, with constant $T_{e} / T_{+}$, to the following scaling

$$
R \propto \frac{(R L)^{1 / 2} n_{g}}{B_{0} \bar{\alpha}^{1 / 2}}
$$

This scaling will be used for comparison between the numerical and analytic solution in section 6.3.2. It should be noted that $R \propto B_{0}^{-1}$, i.e. decreases with $B_{0}$, as found in figure 6.9 .

To complete the description of the discharge equilibrium, the negative ion balance equation is approximated with $n_{-}=\bar{n}_{-}=$const:

$$
\frac{d \Gamma_{-}}{d x}=\nu_{\mathrm{att}} n_{e}(x)-K_{\mathrm{rec}} \bar{n}_{-}\left(\bar{n}_{-}+n_{e}(x)\right)
$$

This equation can easily be integrated since $n_{e}(x)$ is known and all other terms are constants. Using the initial conditions on the negative ions, $n_{-}(0)=n_{n 0}$ and $v_{-}(0)=0$, $\Gamma_{-}(0)=0$ is found, leading to

$$
\Gamma_{-}(x)=\frac{n_{e 0}}{2}\left(\nu_{\mathrm{att}}-K_{\mathrm{rec}} \bar{n}_{-}\right)\left(x+\frac{R}{\pi} \sin \frac{\pi x}{R}\right)-K_{\mathrm{rec}} \bar{n}_{-}^{2} x .
$$

Since $\beta$ is defined as the ratio of the ion velocity at $x=R$ to the ion Bohm velocity, in order to obtain the ion flux, the ion density at $x=R$ is also needed. Using $\alpha_{s}=n_{s}(R) / n_{e 0}$, the negative ion flux can be expressed as $\Gamma_{-}(R)=\beta \alpha_{s} n_{e 0} u_{B+}$. Finally, equating both expressions of the negative ion flux at $x=R$ yields

$$
\Gamma_{-}(R)=\frac{n_{e 0} R}{2}\left[\nu_{\mathrm{att}}-K_{\mathrm{rec}} n_{e 0} \bar{\alpha}(1+2 \bar{\alpha})\right]=\beta \alpha_{s} n_{e 0} u_{B+} .
$$

Since the ion Bohm velocity is the same for both ion species $\left(m_{+}=m_{-}\right.$and $\left.T_{+}=T_{-}\right)$ and $v_{+}(R)=v_{-}(R), \beta$ is the same for positive and negative ions. Once again, it can be seen that the ion flux at $x=R$ is the difference between attachment (first term in square brackets in (6.78)) and recombination (second term in square brackets in (6.78)).

The relation needed between $\alpha_{s}$ and $\bar{\alpha}$ is somewhat cumbersome and the calculation is given in appendix $\mathrm{A}$ :

$$
\begin{aligned}
&-\frac{3 D_{+}}{2} \alpha_{s}=-\frac{3 D_{+}}{2} \bar{\alpha}- \frac{D_{+}-D_{\perp e}}{1+\mu_{\perp e} / \mu_{+}} \bar{\alpha} \ln \left[1+\frac{1+\mu_{\perp e} / \mu_{+}}{2 \bar{\alpha}}\right] \\
&+\left[\nu_{\mathrm{att}}-K_{\mathrm{rec}} n_{e 0} \bar{\alpha}(1+2 \bar{\alpha})\right] \frac{R^{2}}{4}+\left[\nu_{\mathrm{att}}-K_{\mathrm{rec}} n_{e 0} \bar{\alpha}\right] \frac{R^{2}}{\pi^{2}}
\end{aligned}
$$


Rewriting (6.78) as

$$
\beta=\frac{R}{2 \alpha_{s} u_{B+}}\left[\nu_{\mathrm{att}}-K_{\mathrm{rec}} n_{e 0} \bar{\alpha}(1+2 \bar{\alpha})\right]
$$

and substituting for $\alpha_{s}$ from (6.79), equation (6.80) gives an expression for $\beta$ as a function of the parameters $\left\{R L, n_{g}, B_{0} T_{e} / T_{+}, m_{+}\right\}, \bar{\alpha}$ and $n_{e 0} / n_{g}$. With the parameters, $\bar{\alpha}$ and $n_{e 0}$ as inputs, the output parameters $T_{e}$ (in (6.73)), $R$ and $L$ (in (6.74)), and $\beta$ (in (6.80)) can be determined.

For simplicity in obtaining the $\beta$-curves in the $\left(\bar{\alpha}, n_{e 0} / n_{g}\right)$ plane, the expression of $n_{e 0}$ as a function of the parameters, $\bar{\alpha}$ and $\beta$ has to be calculated. Combining (6.78) and (A.9) gives $\alpha_{s}$ as a function of the parameters, $\bar{\alpha}$ and $\beta$,

$$
\begin{aligned}
\alpha_{s}\left[\frac{3 D_{+}}{2}+\frac{\beta u_{B+} R}{2}\right. & \left.+\frac{2 \beta u_{B+} R}{\pi^{2}(1+2 \bar{\alpha})}\right] \\
& =\bar{\alpha}\left[\frac{3 D_{+}}{2}+\frac{D_{+}-D_{\perp e}}{1+\mu_{\perp e} / \mu_{+}} \ln \left(1+\frac{1+\mu_{\perp e} / \mu_{+}}{2 \bar{\alpha}}\right)-\frac{2 \nu_{\mathrm{att}}}{1+2 \bar{\alpha}} \frac{R^{2}}{\pi^{2}}\right] .
\end{aligned}
$$

The expression of $n_{e 0}$, from (6.78), is

$$
n_{e 0}=\frac{\nu_{\mathrm{att}}-2 \beta \alpha_{s} u_{B+} / R}{K_{\mathrm{rec}} \bar{\alpha}(1+2 \bar{\alpha})} .
$$

In the context of thrusters, it is interesting to maximize the outgoing ion flux. Because of the ion density decay for high $\beta$ 's seen in the numerics, maximizing $\beta$ is not equivalent to maximizing the ion flux. Moreover, the expressions of $T_{e}$ in (6.73) and $R$ in (6.74) show that they are independent of $n_{e 0} / n_{g}$. This means that for a given $\bar{\alpha}, R$ is constant, describing one geometry. Therefore, an optimization of $\Gamma_{-}(R)$ as a function of $n_{e 0}$ and for constant $\bar{\alpha}$ is meaningful. The optimized value of $n_{e 0}$ is found from (6.78) by calculating

$$
\frac{d \Gamma_{-}(R)}{d n_{e 0}}=0,
$$

at constant $\bar{\alpha}$. The expression obtained is

$$
\left.n_{e 0}\right|_{\mathrm{opt}}=\frac{\nu_{\mathrm{att}}}{2 K_{\mathrm{rec}} \bar{\alpha}(1+2 \bar{\alpha})} .
$$

Replacing $n_{e 0}$ with its optimized value in (6.78) reveals that half of the negative ions flow to the wall while half recombine in volume (the recombination terms becomes $\frac{1}{2} \nu_{\text {att }}$ ).

Figure 6.11 is a first equivalent to figure 6.7. It shows $n_{e 0} / n_{g}$ as a function of $\bar{\alpha}$ for three values of $\beta(0,0.15$ and 1$)$ and $\left.n_{e 0}\right|_{\text {opt }} / n_{g}$ as a function of $\bar{\alpha}$. As in the numerics, the values of the parameters are $R L=100 \mathrm{~cm}^{2}, p=10 \mathrm{mTorr}, B_{0}=300 \mathrm{G}, T_{e} / T_{+}=20$ and $m_{+}=32$ a.m.u. It can be observed that although $\bar{\alpha}$ is used instead of $\alpha_{0}$, the figure from the numerics and that from the analytical model are very similar, showing three regions in their corresponding plane. For every value of $\left(\bar{\alpha}, n_{e 0} / n_{g}\right)$, there are solutions fulfilling both boundary conditions. To the right of the $\beta=0$ curve, these solutions are for negative $\beta$ 's, meaning that this region is of no interest (the plasma cannot be sustained on its own). To the left of the $\beta=1$ curve is a region where $\beta>1$, meaning that the ion velocities are greater than the ion sound velocity. This situation is possible in the analytic approximation because the inertia terms for the ions were dropped, but is not physical. The region between the $\beta=0$ and $\beta=1$ curves is where all solutions are: both boundary 


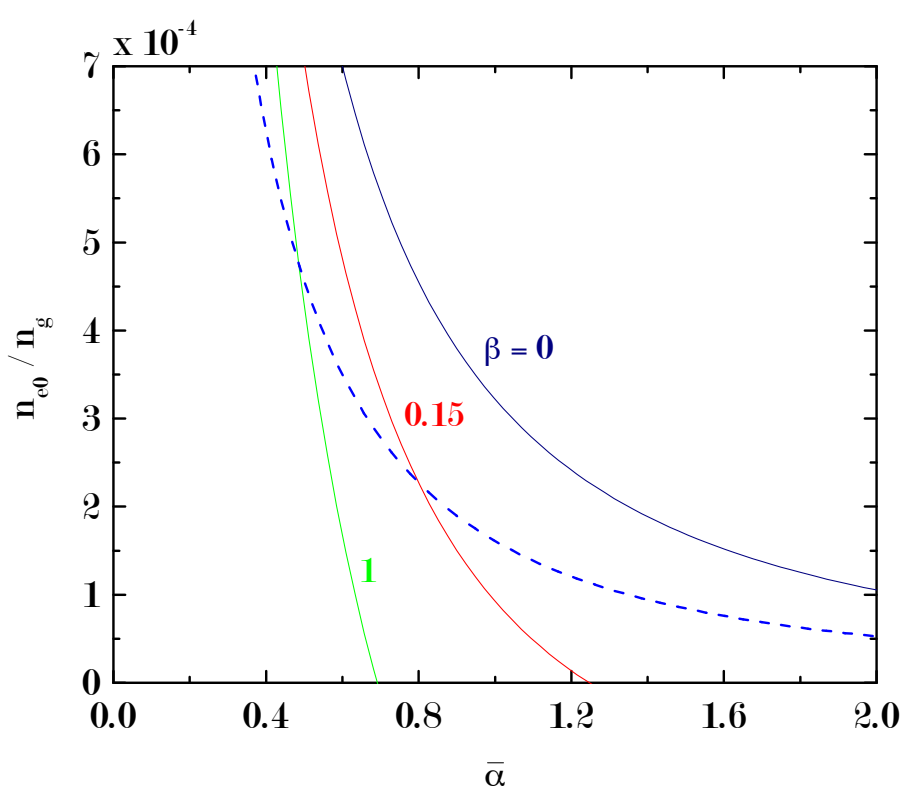

Figure 6.11: $n_{e 0} / n_{g}$ versus $\bar{\alpha}$ for $p=10 \mathrm{mTorr}, B_{0}=300 \mathrm{G}, R L=100 \mathrm{~cm}^{2}$, $T_{e} / T_{i}=20$ and $M_{i}=32$ a.m.u. The upper-through-lower curves are for $\beta=0,0.15$ and 1 respectively; the dashed curve gives $\left.n_{e 0}\right|_{\text {opt }} / n_{g}$.

conditions are fulfilled and $0<\beta<1$. It should be noted that the $\beta=$ const curves do not intersect. Also, the $n_{e 0}(\mathrm{opt}) / n_{g}$ curve shows that the maximum flux is not necessarily obtained for $\beta=1$. As discussed previously, this is due to the fact that for high $\beta$ 's, the edge densities drop to a small value compared to the central densities.

In order to further the comparison between the numerics and the analytical model, the $\left(\alpha_{0}, n_{e 0} / n_{g}\right)$ plane needs to be considered instead of $\left(\bar{\alpha}, n_{e 0} / n_{g}\right)$. For that, the relation between $\alpha_{0}$ and $\bar{\alpha}$ derived in (A.12) is used:

$$
\alpha_{0}=\frac{3}{2} \bar{\alpha}-\frac{1}{2} \alpha_{s}
$$

Figure 6.12 can be easily obtained by using the parametric plot of $n_{e 0} / n_{g}$ as a function of $\bar{\alpha}$ and $\beta$ versus $\alpha_{0}$ as a function of the same variables $\bar{\alpha}$ and $\beta$. The parameters were kept constant. The $\beta$ curves are changed and can now be seen to intersect, as it was seen in the numerics in figure 6.7. For instance, the $\beta=0.15$ and $\beta=1$ curves do so for $\alpha_{0}=0.67$ and $n_{e 0} / n_{g}=4.69 \times 10^{-4}$. The $R$ curves are obtained in the same way as the $\beta$ curves, a parametric plot of $n_{e 0} / n_{g}$ as a functions of $\bar{\alpha}$ and $R$ versus $\alpha_{0}$ as a function of $\bar{\alpha}$ and $R$. In the previous figure (6.11), the $R$ curves were vertical lines since $R$ only depends on $\bar{\alpha}$. Here, the $R$ curves are slanted lines, as expected from (6.85). The fact that $n_{e 0} / n_{g}$ for constant $R$ is a linear function of $\alpha_{0}$ can be derived from (A.9) by replacing $\alpha_{s}$ with its expression in (A.12),

$$
\begin{array}{r}
-\frac{3 D_{+}}{2}\left(3 \bar{\alpha}-2 \alpha_{0}\right)=-\frac{3 D_{+}}{2} \bar{\alpha}-\frac{D_{+}-D_{\perp e}}{1+\mu_{\perp e} / \mu_{+}} \bar{\alpha} \ln \left[1+\frac{1+\mu_{\perp e} / \mu_{+}}{2 \bar{\alpha}}\right] \\
+\left[\nu_{\mathrm{att}}-K_{\mathrm{rec}} n_{e 0} \bar{\alpha}(1+2 \bar{\alpha})\right] \frac{R^{2}}{4}+\left[\nu_{\mathrm{att}}-K_{\mathrm{rec}} n_{e 0} \bar{\alpha}\right] \frac{R^{2}}{\pi^{2}} .
\end{array}
$$

As it was seen, $R$ constant means that $\bar{\alpha}$ and $T_{e}$ are constant; all terms in equation (6.86) are constants. The relation between $n_{e 0} / n_{g}$ for constant $R$ and $\alpha_{0}$ is indeed linear, and with a negative slope. 


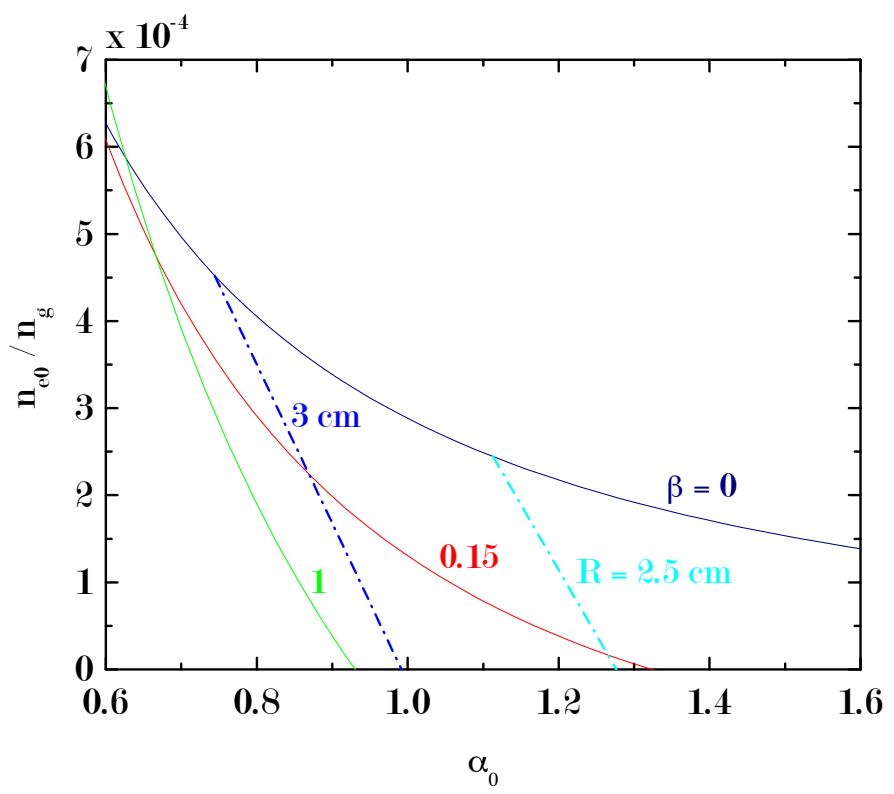

Figure 6.12: $n_{e 0} / n_{g}$ versus $\alpha_{0}$ for $p=10 \mathrm{mTorr}, B_{0}=300 \mathrm{G}, \mathrm{RL}=100 \mathrm{~cm}^{2}$, $T_{e} / T_{+}=20$ and $m_{+}=32$ a.m.u. The upper-through-lower solid curves are for $\beta=0,0.15$ and 1 respectively; the dot-dashed curve gives the curves of constant $R=3 \mathrm{~cm}$ and $R=2.5 \mathrm{~cm}$.

Finally, the evolution of $\alpha_{s} / \alpha_{0}$ as a function of $\alpha_{0}$ for the same parameters is shown in figure 6.13 . For small $\beta$ 's, the negative ion density at the edge is rather flat, but as soon as $\beta=0.15$, the edge density drops with values around a quarter of the central density for $\beta=1$.

\subsubsection{Comparison with Numerics, Parameter Scaling}

\section{The input and output parameters}

Besides the main parameters $\left\{R L, n_{g}, B_{0} T_{e} / T_{+}, M_{+}\right\}$, the input and output parameters in the two solution methods are somewhat different. In the numerics, the inputs are the initial, or central, electronegativity $\alpha_{0}$ and the initial electron density $n_{e 0}$. The outputs are the length of the system $R$, the electron temperature $T_{e}$, and $\beta$, the ion velocity at $x=R$ normalized to the ion sound velocity. As a result, $T_{e}$ and $R$ are functions of both inputs. In the analytical model, the easiest input parameters are the average electronegativity $\bar{\alpha}$ and $\beta$. It was shown in the calculations that outputs $T_{e}$ and $R$ are only functions of $\bar{\alpha}$. The last output $n_{e 0}$ is a function of both inputs. However, in the analytical model, transformations can be used to obtain the problem as functions of $\alpha_{0}$ and $n_{e 0}$.

The evolution of $R$ and $T_{e}$ as functions of $\alpha_{0}$ (in the numerics) and $\bar{\alpha}$ (in the analytical model) for constant $n_{e 0} / n_{g}$ are the same:

$$
\text { When }\left(\alpha_{0}, \bar{\alpha}\right) \nearrow, \quad T_{e} \nearrow \text { and } R \searrow \text {. }
$$

Independent of $n_{e 0}$ in the analytical model, $T_{e}$ and $R$ do depend on the initial electron density in the numerics, with the following for constant $\alpha_{0}$

$$
\text { When } n_{e 0} \nearrow, \quad T_{e} \searrow \text { and } R \searrow \text {. }
$$




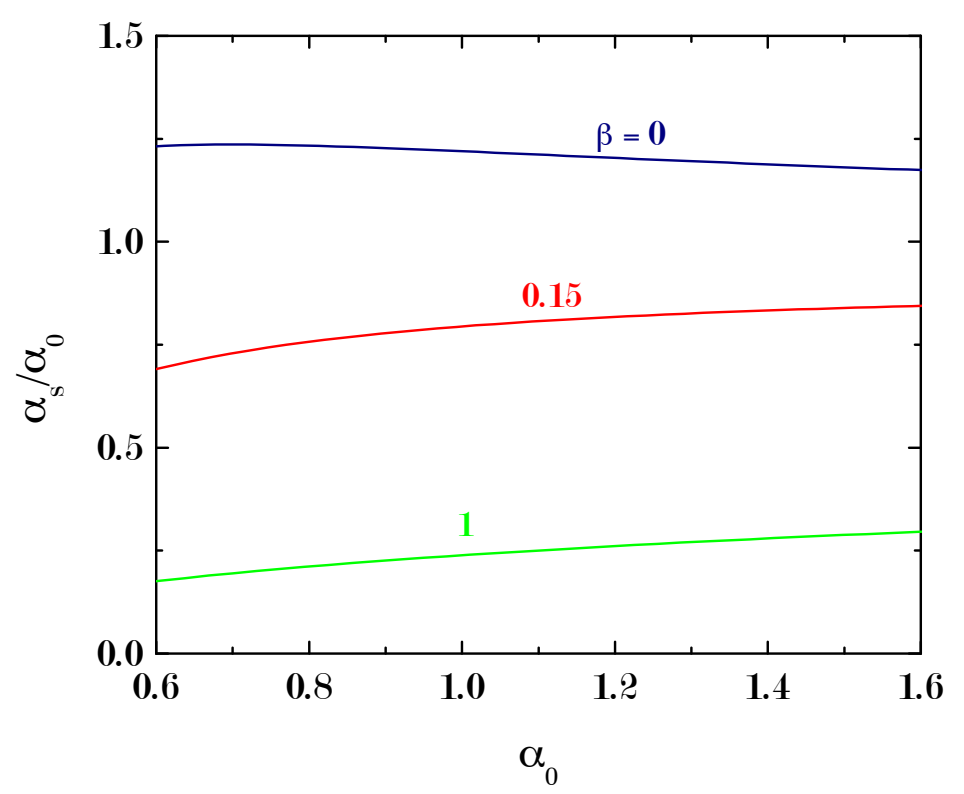

Figure 6.13: $\alpha_{s} / \alpha_{0}$ versus $\alpha_{0}$ for $p=10 \mathrm{mTorr}, B_{0}=300 \mathrm{G}, R L=100 \mathrm{~cm}^{2}$, $T_{e} / T_{i}=20$ and $M_{i}=32$ a.m.u.

\section{The negative ion density profile}

The main assumption to solve the electron balance equation in the analytical model is the constant negative ion density. It was seen that this assumption is valid for small values of $\beta$, but for values of $\beta$ near 1 , the edge density is lower than the central density by as much as a factor of 5 (see figure 6.6). Therefore, there are two regions: for small $\beta$ 's, both models should be in good agreement, but for high $\beta$ 's, there should be discrepancies. This can be seen when comparing the $\left(\alpha_{0}, n_{e 0} / n_{g}\right)$ planes in both cases (figures 6.7 and 6.12): the $\beta=0$ curves are quite close but the $\beta=1$ curves are significantly different.

However, $n_{-}=\bar{n}_{-}$is not used in the calculation of $\beta$. The value of the edge electronegativity is derived as a function of the input parameters. Figure 6.13, showing the ratio of the edge electronegativity $\alpha_{s}$ to the the central electronegativity $\alpha_{0}$ as a function of the central electronegativity $\alpha_{0}$, confirms the evolution of the negative ion density profile versus $\beta$.

\section{The inertia terms}

The inertia terms $\nabla\left(n_{i} v_{i} v_{i}\right)$ and $\nabla\left(n_{n} v_{n} v_{n}\right)$ were dropped in the analytical model in order to simplify the problem. Between this and the assumption of quasi-neutrality, there is no way in this model to know whether a solution is physical or not without using an additional condition. This condition was chosen to be on the edge ion velocity, requiring

$$
0<\beta<1
$$

In the numerics, although quasi-neutrality is also assumed, the presence of the inertia terms ensures that, if the parameters lead to a singularity in velocities, the simulation is stopped. All results presented were done with those conditions. In order to see their effect, the inertia terms were dropped in a few characteristic simulations. For small values of $\beta$, the differences between cases with and without the inertia terms are negligible. For higher values of $\beta$, the difference increases slowly, because the ion velocities are small 
over most of the simulation, implying that the inertia terms are of minor importance. Only close to $x=R$ do the inertia terms play a role in the simulations, leading to mathematical singularities (diverging velocities). This implies the formation of a sheath since quasi-neutrality is no longer fulfilled.

\section{The $\left(\alpha_{0}, n_{e 0} / n_{g}\right)$ plane}

By using the calculated relation between the average electronegativity $\alpha_{s}$ and the central electronegativity $\alpha_{0}$ for the analytical model, the space of solutions can be represented in the $\left(\alpha_{0}, n_{e 0} / n_{g}\right)$ plane for both calculations. The visual agreement between figures 6.7 and 6.12 is good, whether when comparing the $\beta$ or $R$ curve loci.

Near the $\beta=0$ limit, both models are extremely close. For instance, with $n_{e 0} / n_{g}=$ $2.75 \times 10^{-4}$, this limit is reached for $\alpha_{0}=1.0$ in the numerics while the analytical model predicts that it is reached for $\alpha=1.031$. As was seen previously, the negative ion density profile is flat for values of $\beta$ close to 0 , which validates the $n_{n}=\bar{n}_{n}$ assumption in the model. Moreover, the inertia terms, which have little effect, are negligible with $\beta \approx 0$.

Near the $\beta=1$ limit, some differences can be seen. For $n_{e 0} / n_{g}=2.75 \times 10^{-4}$, the limit is approached for $\alpha_{0}=0.828$ in the numerics, but for $\alpha_{0}=0.754$ in the analytical model. Here, the assumption that the negative ion density is constant does not hold, with the effect that the electron density profile calculated in the analytical model is significantly in error. The inertia terms also play a role near this limit.

The $R$ curves are slightly shifted towards lower values of $\alpha_{0}$ in the analytical model. Also, the $R$ curves in the numerics are not as linear with $\alpha_{0}$.

\section{The Scaling of the optimized $\Gamma_{-}(R)$}

In the context of thrusters, the main output parameter of interest is the flux of ions. For PEGASES, it is the negative ion flux that needs to be optimized since the positive ion flux goes down to the value of the negative ion flux as the electron flux goes to zero at $x=R$. The negative ion flux was calculated in (6.78)

$$
\Gamma_{-}(R)=\frac{n_{e 0} R}{2}\left[\nu_{\mathrm{att}}-K_{\mathrm{rec}} n_{e 0} \bar{\alpha}(1+2 \bar{\alpha})\right]
$$

while the optimized electron density for this equation was calculated in (6.84)

$$
n_{e 0}(\mathrm{opt})=\frac{\nu_{\mathrm{att}}}{2 K_{\mathrm{rec}} \bar{\alpha}(1+2 \bar{\alpha})} .
$$

Combining these two equations yields the expression of the optimized negative ion flux

$$
\left.\Gamma_{-}(R)\right|_{\mathrm{opt}}=\frac{1}{8} \frac{\nu_{\mathrm{att}} R}{K_{\mathrm{rec}} \bar{\alpha}(1+2 \bar{\alpha})} .
$$

The expression of $\bar{\alpha}$ can be approximated from (6.74) since $T_{e} / T_{+} \gg 1$

$$
\bar{\alpha}=\frac{\pi^{2}}{4} \frac{L}{R} \frac{D_{\perp e}}{D_{+}}\left(\frac{T_{+}}{T_{e}}\right)^{1 / 2} .
$$

With a high enough magnetic field, $D_{\perp e}$ can be approximated with $\left(e T_{e} \nu_{e}\right) /\left(m_{e} \omega_{c e}^{2}\right)$, while $D_{+}=\left(e T_{+}\right) /\left(m_{+} \nu_{+}\right)$(unmagnetized ions), giving the following scalings

$$
D_{\perp e} \propto \frac{n_{g}}{B_{0}^{2}} \quad \text { and } \quad D_{+} \propto \frac{1}{n_{g}} .
$$


The scaling of $\bar{\alpha}$ can thus be written as

$$
\bar{\alpha} \propto \frac{L}{R} \frac{n_{g}^{2}}{B_{0}^{2}} .
$$

Depending on $\bar{\alpha}$, there are two regimes: low and high electronegativity

$$
\begin{aligned}
& \bar{\alpha}<\frac{1}{2} \quad \Rightarrow \quad \frac{1}{\bar{\alpha}(1+2 \bar{\alpha})} \simeq \frac{1}{\bar{\alpha}} \\
& \bar{\alpha}>\frac{1}{2} \quad \Rightarrow \quad \frac{1}{\bar{\alpha}(1+2 \bar{\alpha})} \simeq \frac{1}{2 \bar{\alpha}^{2}} .
\end{aligned}
$$

The scaling of the negative ion flux for low electronegativity is thus

$$
\left.\Gamma_{-}(R)\right|_{\mathrm{opt}} \propto \frac{K_{\mathrm{att}}}{K_{\mathrm{rec}}} \frac{B_{0}^{2}}{n_{g}} \frac{R^{2}}{L},
$$

and for high electronegativity

$$
\left.\Gamma_{-}(R)\right|_{\text {opt }} \propto \frac{K_{\text {att }}}{K_{\text {rec }}} \frac{B_{0}^{4}}{n_{g}^{3}} \frac{R^{3}}{L^{2}} .
$$

In both cases, it can be seen that the parameters to increase the negative ion flux are the attachment rate (increasing function) and the magnetic field strength (increasing function). Although the negative ion flux decreases as the pressure (neutral density) is increased, the latter plays a stronger role at high electronegativity. As expected, the negative ion flux is a decreasing function of the recombination rate.

\section{The scaling of $R$}

With the assumption that the negative ion density is constant, it was found in the analytical model that

$$
R \propto \frac{(R L)^{1 / 2} n_{g}}{B_{0} \bar{\alpha}^{1 / 2}} .
$$

In the numerics, the study $R$ as a function of $\alpha_{0}$ shows a behavior like $R \propto \alpha_{0}^{-c}$ with $c$ a positive constant. Plotting $R$ versus $\alpha_{0}^{-1}$ does not result in a linear curve, but the curvature shows that $c<1$. Therefore, the scaling of $R$ with $\left(\bar{\alpha}, \alpha_{0}\right)$ is consistent between the two models.

Since only three values of $R L$ were studied numerically, the comparison is limited. However, plotting $R$ versus $R L$ for different parameter values does show a linear behavior.

Finally, figure 6.9 shows how $R$ scales as a function of $B_{0}$ for the numerics. The profile of the curve is consistent with $R \propto B_{0}^{-1}$.

\subsection{Model Limitations}

\subsubsection{Symmetrical Ion-Ion Plasma}

The choice was made to consider a plasma composed of only one species of positive ion and one species of negative ion, with the masses of the ions set to the same value (32 a.m.u.) and the ion temperatures set equal $\left(T_{+}=T_{-}=T_{e} / 20\right)$. This allowed a zero potential 
gradient at $x=R$ to be found; with a zero electric field, there is no sheath at the edge of the plasma.

A few cases were run with $m_{+} \neq m_{-}$or $T_{+} \neq T_{-}$. In these cases, the wanted solution fulfilling both boundary conditions can still be found for a range of input parameters. It was seen previously that when the boundary conditions are fulfilled, the ion densities and fluxes are equal at $x=R$. It was seen numerically that with an asymmetrical ion-ion plasma, either masses or temperatures unequal, these equalities are obtained with a nonzero electric field at the edge: the symmetrical extraction of ions is no longer possible. One of the gases studied in experiments is oxygen, with $\mathrm{O}_{2}^{+}$and $\mathrm{O}^{+}$as positive ions and $\mathrm{O}^{-}$as negative. The value of $\zeta=m_{+} / m_{-}$, therefore, may reach 2 : an ion-ion plasma obtained in the conditions described in the model would be asymmetrical.

The situation becomes even more complex in the case of sulfur hexafluoride, where there are several types of positive ions and several types of negative ions. One could use the described model with average masses and temperatures for the positive ions and the negative ions, but it would also result in an asymmetrical plasma since the average masses and/or the temperatures would be different.

\subsubsection{Gas Electronegativity}

In order to be as close as possible to the first experiments run, oxygen was chosen as feedstock gas. However, oxygen is a weakly electronegative gas, which makes it a less than ideal candidate for ion-ion plasma creation since the limiting process for such a purpose is the negative ion production. It should also be noted that for thrust purposes, the mass of the oxygen ions (16 and 32 a.m.u.) is quite low, especially compared to xenon ions (131 a.m.u.), which are the typical ions used in classical electropositive thrusters.

This reduced electronegativity is the major reason why the ionization fraction found in the results is so low $\left(\sim 10^{-4}\right)$. Indeed, it was calculated in (6.98) and (6.99) that the optimized negative ion flux scales as the square of the attachment rate. With the low attachment rate of oxygen, only low densities and fluxes can be obtained. For a thruster, the desired ionization fraction is, of course, as close as possible to 1, with any neutral entering the thruster turned into an ion and providing thrust. The ionization fraction found in the model, therefore, is extremely low.

The aim of the simulations was to be comparable to the experiment, with a radius of $R=2.3 \mathrm{~cm}$, which gave the value of the normalization $R_{0}=2.5 \mathrm{~cm}$. The $R$ curve of $R=2.5 \mathrm{~cm}$, therefore, represents the experiment. It should be noted that although the described regime is at very low electron density, solutions with $R=2.5 \mathrm{~cm}$ and fulfilling both boundary conditions were found, meaning that obtaining an ion-ion plasma in the given geometry is possible, albeit in a different regime than hoped for (low ionization fraction $\left.n_{e 0} / n_{g}\right)$.

There are two ways of simulating higher electronegativities, either change all parameters for a more electronegative gas ( $I_{2}$ for instance), or only modify the value of $K_{\text {att }}$ used in the simulations (e.g. multiplying it by a constant factor). The first solution requires all parameter values to be changed to describe a new feedstock gas, as well as a completely new search for solutions. The second one is a lot easier to implement, especially with a gradual increase of $K_{\text {att }}$ : the evolution of the solution space could be tracked and such evolution could be understood in regard to the increase of the attachment factor. The end value of the attachment factor could be set to $K_{\text {att }}\left(I_{2}\right) / K_{\text {att }}\left(O_{2}\right)$ for instance. These modifications were not done due to the lack of time. 


\subsubsection{Solutions with $n_{e}(R) \neq 0$}

In order to reach complete electron confinement, the boundary conditions required the electron density and the electron flux to be zero at $x=R$. As the numerical integration procedure was described (6.2.4), it was found that the boundary condition on the electron flux could be fulfilled for a range of electron temperatures, with either the maximum or both extrema fulfilling the other boundary condition on the electron density. These solutions were not directly investigated, but data is available from the shooting method on the electron temperature (figures 6.3 and 6.4). It should be noted that the additional condition $0<\beta<1$ (self-sustained and sheath-free solutions) is also fulfilled with $n_{e}(R) \neq 0$. Although $v_{e}(R)=0$ is obtained, it is the fluid velocity and not the velocity of all electrons: with the assumption of a Maxwellian velocity distribution function, half the electrons have a positive velocity while the other half has a negative velocity. The described situation, therefore, is not one of electron confinement. As a consequence, a sheath should be found where the electron flux goes to zero. The amplitude of this sheath determines whether the extraction of both ion species is possible. However, as the electrons are magnetized, their diffusion is impeded, meaning that the negative ions should still dominate the plasma equilibrium.

For lower electron temperatures than the ranges found for $n_{e}(R)=0$, solutions can be found with a non-zero electron flux, although it is small compared to the ion fluxes. These solutions were not investigated, but correspond to a case where the magnetic confinement of electrons is not attained. As a complete confinement cannot be done in experiments, due to detachment for instance, these solutions could prove to be interesting when comparing with experiments.

\subsubsection{Constant $T_{e}$}

The assumption of constant $T_{e}$, as well as constant $T_{+}, T_{-}$and $T_{e} / T_{+}$, was made to facilitate the integration of the equations. For these temperatures to be variables of the problem, the third moment of the fluid equation should be considered for all species, amounting to nine equations, with difficult boundary conditions on heat transfer to be set. However, the electron temperature profiles in the first PEGASES thruster prototype (figure 4.13 in section 4.4) show that, in fact, there are two electron temperature regions: the electronegative core has temperatures in the range of $10 \mathrm{~V}$, while near the periphery, the temperature is around 2-3 V. Assuming constant $T_{e}$ is thus a very strong assumption.

The consequence is that the same $T_{e}$ provides the ionization (positive ion and electron creation, $K_{\mathrm{iz}}$ ) and the attachment (negative ion creation, $K_{\mathrm{att}}$ ). The issue lies with the fact that high ionization is obtained for high $T_{e}$, while high attachment for low $T_{e}$ (as low as $T_{e}=0 \mathrm{~V}$ in the case of $I_{2}$ ). The solutions are thus compromises between low-medium ionization and low-medium attachment: the constant $T_{e}$ assumption is one of the reasons why such low ionization fractions were found in the results.

Since there are measured electron temperature profiles, it is possible to modify the model to take it into account: a given $T_{e}(x)$ can be used instead of $T_{e}$. For instance, the electron temperature can be defined as

$$
T_{e}(x)=T_{e 0} \times f(x) \quad \text { for } \quad x \in[0,+\infty[,
$$

with $f(x)$ based on the electron temperature profile measurements, leaving $T_{e 0}$ as the eigenvalue to solve for. 
It should be noted that equation (6.25) becomes

$$
\Gamma_{e}(R)=n_{g} \int_{0}^{R}\left(K_{\mathrm{iz}}-K_{\mathrm{att}}\right) n_{e} d x .
$$

The boundary condition $\Gamma_{e}(R)=0$ may be obtained with $K_{\mathrm{iz}}>K_{\mathrm{att}}$ in the high temperature region, and $K_{\mathrm{iz}}<K_{\text {att }}$ in the low temperature, without the need for end losses.

\subsubsection{Non-Uniform Neutral Density}

The neutral density $n_{g}$ was assumed constant to solve the fluid equations. However, the assumption is only valid at very low ionization fraction.

In the case of thrusters, the desired ionization is as close as possible to 1 . This means that every single neutral is ionized and yields an electron and an ion. The steady-state neutral density, therefore, is close to zero in the case of the desired ionization fraction. This needs to be taken into account.

Although the ionization fraction obtained in the results was small (around $10^{-4}-10^{-3}$ ), the effect called neutral depletion $[54,55,56,57,58]$ must play a role in the plasma equilibrium. As the electron temperature is much higher than that of the neutrals, the electron pressure can become comparable to the neutral pressure even at low ionization fraction $\left(\sim 10^{-2}\right)$. The neutrals are thus pushed away from the region of high electron temperature As a result, the neutral density is not uniform and depends on the position. 


\section{Chapter 7}

\section{Ion Extraction Stage}

\section{Contents}

7.1 Description of the Problem .............. 138

7.1 .1 High Voltage Sheath . . . . . . . . . . . . . . . . . . 138

7.1 .2 Low Voltage Sheath . . . . . . . . . . . . . . . . . . . . 139

7.2 Basic Fluid Model Study . . . . . . . . . . . . . 139

7.2 .1 Motivation ........................ 139

7.2 .2 Assumptions . . . . . . . . . . . . . . . . . 140

7.2 .3 Poisson and Model Equations . . . . . . . . . . . . . . . . 141

7.2 .4 Ion-Ion Plasma . . . . . . . . . . . . . . . . . . . 147

7.3 PIC Simulations . . . . . . . . . . . . . 147

7.3.1 Description of the PIC Simulations . . . . . . . . . . . . 147

7.3 .2 Results . . . . . . . . . . . . . . . . . . . . . 148

7.3 .3 PIC Limitations . . . . . . . . . . . . . . . . . . 151

7.4 Kinetic Model .................... 151

7.4 .1 Assumptions . . . . . . . . . . . . . . . . 151

7.4.2 Initial Distribution Function Requirements . . . . . . . . . 153

7.4 .3 Equation of the Model . . . . . . . . . . . . . . . . . 154

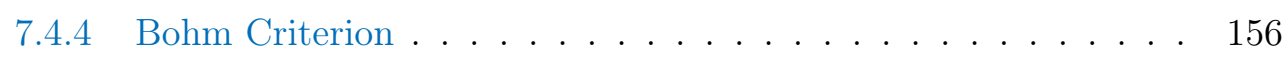

7.4 .5 High Potential . . . . . . . . . . . . . . . . . . 158

$7.4 .6 \quad$ Floating Potential . . . . . . . . . . . . . . . . . . . 158

7.5 Conclusions . . . . . . . . . . . . . 162

The third and final stage of the PEGASES thruster is the ion extraction and acceleration. To realize it, two designs were considered: a magnetic divergence similar to VASIMIR (see section 1.1.4) or biased grids as in ion thrusters (see section 1.1.3). As an easier design to implement, biased grids were chosen over the magnetic divergence. Since a plasma adapts to a biased electrode with a sheath, a small region near the electrode where the potential difference between the plasma potential and the electrode potential is localized, understanding the scaling laws of sheaths for ion-ion plasmas is crucial to the actual implementation of the design. 
In order to look into the problem, a set of basic fluid models were first studied for two cases, depending on what assumption is made for each species. PIC simulations were then run for an ion-ion plasma in the steady-state regime to characterize the response of this plasma to direct-current biased electrodes. A kinetic model for the sheath was then developed to understand the velocity distribution of both species, yielding results for a Bohm criterion and scaling laws for high and small voltage sheaths.

\subsection{Description of the Problem}

The situation is a priori very different from an electron-positive ion plasma where the two involved species have different properties due to the mass ratio between the two $\left(5.8 \times 10^{4}\right.$ in oxygen). Electrons are characterized by a high temperature, which results in a fast diffusion. Positive ions are characterized by a low temperature and a slow diffusion. For ion-ion plasmas, the two involved species have either the same or comparable masses. For example in oxygen, the main ions are $\mathrm{O}_{2}^{+}$and $\mathrm{O}^{-}$, which means a mass ratio of 2 . The diffusions, therefore, are similar and should result in a specific behavior near a biased electrode. Two cases of biased electrode are considered: the high voltage sheath where ions are accelerated to high velocities, and the low voltage sheath which corresponds to an electrode at the floating potential.

\subsubsection{High Voltage Sheath}

The first aspect to study about sheaths in an ion-ion plasma is the plasma response to a high potential difference imposed by grids. The aim is to accelerate ions to a velocity as high as possible. In order to optimize the thrust, all ions need to be accelerated in the direction of the thrust. This is due to the fact that an ion, accelerated in a direction which makes an angle with the direction of thrust, only contributes to the total thrust with the cosine of this angle as a factor. An ion entering the sheath is accelerated in the direction perpendicular to the tangent of the sheath at that point. In the case of a planar electrode, all ions are accelerated in the same direction, but all ions are also collected.

This is why grids are used, as they let part of the ions through: the ratio of ions passing through to all ions accelerated toward the sheath is called grid transparency. The main parameters of a grid are the hole size and number of holes per area. These are optimized for a maximum transparency and a sheath edge as planar as possible. Figure 7.1 shows the sheath profile in front of a grid with holes that are too big. It can be seen that some ions are correctly accelerated in the direction of thrust, perpendicular to the grid. However, the non-planar sheath profile results in two limitations. Some of the ions that pass through the grid are not accelerated in the direction of thrust, which means a reduced thrust. The rest of the ions, which could have gone through the grid, are accelerated toward the grid and collected: they are lost for the thrust.

What determines the sheath profile in front of a grid is the sheath size, distance between the sheath entry point and the grid. The larger it is, the more planar the sheath profile is. The sheath size as a function of operating parameters, potential difference and ion density for instance, needs to be known. The size of the grid holes can then be determined. 


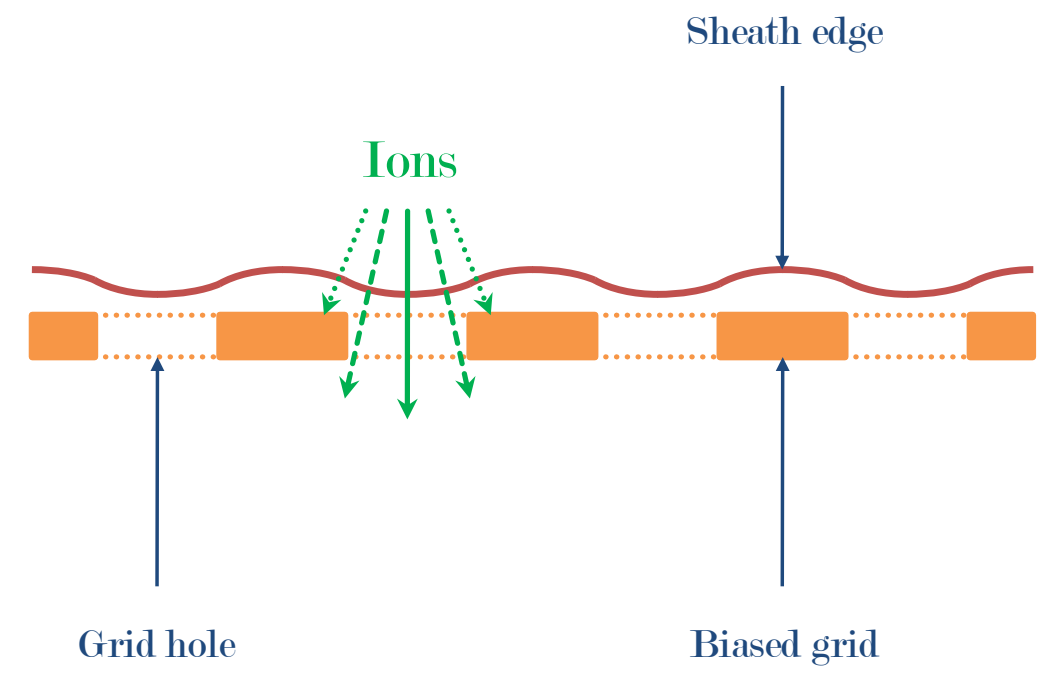

Figure 7.1: Sheath edge near a biased grid. Ions are accelerated perpendicularly to the sheath tangent where they enter the sheath. A part of the ions is accelerated in the direction of thrust (solid line), another is accelerated with an angle (dashed line), and the last part is collected by the grid (dotted line).

\subsubsection{Low Voltage Sheath}

As the walls of the PEGASES thruster are made of dielectric material, the total current at these walls needs to be zero. The wall potential is thus the floating potential. The potential difference between the plasma and the walls is the difference between plasma and floating potentials $V_{p}-V_{f}$. A small sheath, therefore, is present in front of these walls. We shall see that for an ion-ion plasma with equal masses and temperatures, the plasma and floating potentials are equal since the ion currents cancel each other without the need to accelerate one of the ion species and decelerate the other.

With a mass ratio different than 1, as in oxygen, for example, with mass ratio of 2 between the positive ion $\mathrm{O}_{2}^{+}$and the negative ion $\mathrm{O}^{-}$, the ion-ion plasma is no longer symmetrical, which means that a small potential difference between the plasma and the wall is needed for the total current to be zero. To understand the potential profile in the thruster cylinder, the floating potential as a function of mass ratio is needed.

\subsection{Basic Fluid Model Study}

\subsubsection{Motivation}

In an electron-positive ion plasma, the sheath can be described with a simple fluid model where the electrons are assumed to be in Boltzmann equilibrium while the inertia dominates the dynamics of positive ions. Because of the small electron mass compared to that of the positive ions, the inertia effect is neglected for electrons. The pressure effect for the positive ions is neglected since their temperature is much smaller than that of the electrons. The Boltzmann equilibrium assumption for the electrons is further justified as they are the confined species. This holds true whether there is a low (floating potential) or high (ion extraction) voltage sheath.

In the case of an ion-ion plasma, both species have comparable masses and temper- 
atures. It would be logical that the dominating effect is the same for the positive and negative ions. The case where both species are inertia dominated does not apply to the range of pressures and densities of the plasmas considered for the PEGASES thruster. It is thus the pressure dominated assumption that should describe the plasma equilibrium. However, one species is confined while the other is accelerated. This creates an asymmetry in the problem. The sheath equilibrium, therefore, should be an intermediate between two extreme cases: the electron-positive ion one, and the symmetrical situation where the thermal force (pressure gradient) dominates.

These two cases are investigated, with emphasis on the scaling laws that can be derived.

\subsubsection{Assumptions}

A one-dimensional ion-ion plasma composed of two species, one positively charged $(+)$ and the other negatively charged $(-)$, is considered with the first two moments of the fluid equations in rectangular coordinates. In order to keep it simple, both ion species are assumed to be singly charged. The choice is made to accelerate positive ions and retard negative ions. This means that the electric field is positive, i.e. the potential gradient is negative.

The initial conditions are chosen as follows

$$
n_{+}(0)=n_{0}, \quad n_{-}(0)=\alpha_{d} n_{0}, \quad v_{+}(0)=u_{0}, \quad v_{-}(0)=\alpha_{v} u_{0}, \quad \varphi(0)=0 .
$$

There are thus two parameters, the ratio of initial densities $\alpha_{d}$ and the ratio of initial fluid velocities $\alpha_{v}$. Quasi-neutrality is usually assumed in the plasma region where the potential gradient is small. In the sheath, however, quasi-neutrality is no longer fulfilled. The pre-sheath is the region in-between, where quasi-neutrality is usually assumed. As the potential structure for ion-ion plasmas is to be determined, the parameter $\alpha_{d}$ allows us to consider cases where quasi-neutrality is not fulfilled at the sheath edge. The parameter $\alpha_{v}$ is needed as electrons and positive ions are known to have different velocities.

As the source term is assumed to be zero, the continuity equation (described in section 2.1.2) is the same for both species and can be written as

$$
\nabla\left(n_{ \pm} v_{ \pm}\right)=0
$$

Combining this equation with the initial conditions (7.1) gives the values of the constant fluxes

$$
n_{+} v_{+}=n_{0} v_{0} \quad \text { and } \quad n_{-} v_{-}=\alpha_{d} \alpha_{v} n_{0} v_{0} .
$$

The plasma and the sheath are assumed to be collisionless, which leaves three terms in the momentum conservation equation (described in section 2.1.2): the pressure, the electric force and the inertia

$$
e T_{ \pm} \nabla n_{ \pm} \pm e n_{ \pm} \nabla \varphi+m_{ \pm} \nabla\left(n_{ \pm} v_{ \pm}^{2}\right)=0 .
$$

In order to simplify the problem further, only two terms are kept, with the electric force as one of them. Two hypotheses can thus be defined:

- Warm hypothesis: the inertia term is neglected as the species is assumed to be massless, resulting in a balance between pressure and electric force,

- Cold hypothesis: the pressure term is neglected as the species temperature is assumed to be zero, resulting in a balance between inertia and electric force. 
The warm hypothesis corresponds to a Boltzmann equilibrium, an assumption usually made for electrons in an electron-positive ion plasma. The cold hypothesis is usually made for positive ions in a low pressure electron-positive ion plasma. As negative ions are confined in a standard electronegative discharge, the question whether they should be considered in Boltzmann equilibrium is still open [74, 21].

For each hypothesis, the density can be expressed as a function of the potential from the momentum conservation equation.

\section{Warm Hypothesis}

Here, equation (7.4) can be rewritten as

$$
\nabla\left(\ln n_{ \pm}\right)=\mp \frac{1}{T_{ \pm}} \nabla \varphi
$$

Using the initial conditions (7.1) to integrate between 0 and $x$ yields the densities

$$
n_{+}=n_{0} e^{-\varphi / T_{+}} \quad \text { and } \quad n_{-}=\alpha_{d} n_{0} e^{\varphi / T_{-}} .
$$

\section{Cold Hypothesis}

With this hypothesis, the momentum conservation equation (7.4) can be written as

$$
\nabla v_{ \pm}^{2}=\mp \frac{2 e}{m_{ \pm}} \nabla \varphi
$$

The expression of the velocities is obtained by integrating this equation with the initial conditions (7.1) between 0 and $x$, and assuming that they are positive

$$
v_{+}=\sqrt{u_{0}^{2}-\frac{2 e}{m_{+}} \varphi} \text { and } v_{-}=\sqrt{\alpha_{v}^{2} u_{0}^{2}+\frac{2 e}{m_{-}} \varphi}
$$

There are thus two conditions on the potential that depend on the initial velocities

$$
\varphi<\frac{m_{+} u_{0}^{2}}{2 e} \text { and } / \text { or } \varphi>-\frac{m_{-} \alpha_{v}^{2} u_{0}^{2}}{2 e} .
$$

The densities can be easily obtained from equation (7.3)

$$
n_{+}=\frac{n_{0}}{\sqrt{1-\frac{2 e}{m_{+} u_{0}^{2}} \varphi}} \text { and } n_{-}=\frac{\alpha_{d} n_{0}}{\sqrt{1+\frac{2 e}{m_{-} \alpha_{v}^{2} u_{0}^{2}} \varphi}} .
$$

\subsubsection{Poisson and Model Equations}

As the model focuses on the sheath, quasi-neutrality cannot be assumed. The Poisson equation is used to solve for the potential

$$
-\varepsilon_{0} \Delta \varphi=e\left(n_{+}-n_{-}\right) .
$$

Since the densities can be expressed as functions of the potential for either hypothesis, the model comes down to one equation.

Two extreme cases were thus studied: 
- Warm-Warm: both ion species are in Boltzmann equilibrium,

- Cold-Warm: cold positive ions and warm negative ions.

In each case, equation (7.11) is linearized to obtain characteristic lengths, and integrated numerically to observe the behavior as parameters are varied. Although a linearized equation of the model is only valid for small values of the potential, characteristic values that also govern the solutions for bigger values of the potential can be extracted. In order to integrate the model equation, the initial electric field, which should be zero in the plasma approximation, is set to a very small positive value $\left(5 \times 10^{-5}\right.$ for the normalized electric field) for the sheath to occur with positive ions accelerated and negative ions decelerated. Otherwise, all parameters stay constant as there is no initial gradient.

\section{Warm-Warm Case}

The equation of the model is obtained by combining the expression of the densities from equation (7.6) and the Poisson equation (7.11)

$$
-\varepsilon_{0} \Delta \varphi=e n_{0}\left(e^{-\varphi / T_{+}}-\alpha_{d} e^{\varphi / T_{-}}\right) .
$$

Once linearized, this equation becomes

$$
-\frac{\varepsilon_{0} T_{+}}{e n_{0}} \Delta \varphi=\left(1-\alpha_{d}\right) T_{+}-\left(1+\alpha_{d} \frac{T_{+}}{T_{-}}\right) \varphi
$$

One can see that the Debye length, defined as

$$
\lambda_{D+}=\sqrt{\frac{\varepsilon_{0} T_{+}}{e n_{0}}},
$$

is the characteristic length for this equation, which can be rewritten as

$$
\lambda_{D+}^{2} \Delta \varphi-\left(1+\alpha_{d} \frac{T_{+}}{T_{-}}\right) \varphi=\left(\alpha_{d}-1\right) T_{+} .
$$

As this equation is a second order ordinary differential equation, the type of solution is determined by the sign of the factor in front of $\varphi$. Here, with a negative sign, solutions are exponentials. However, the linearized equation is not enough to know whether the exponential solution is diverging or converging. To determine this, one has to look at the full equation (7.12). Starting from zero and quasi-neutrality $\left(\alpha_{d}=1\right)$, the potential can either become positive or negative. If it becomes positive, then its second derivative is also positive, which increases the potential. The opposite situation is symmetrical, i.e. a negative potential results in a decrease in potential. Therefore, solutions are diverging exponentials. With a zero electric field, all variables remain constant, which is why a nonzero initial electric field is needed, and decides whether the potential is positive (negative electric field) or negative (positive electric field). The numerical integration shown in figure 7.2 confirms that. As the potential is negative, the positive ion density and negative ion velocity increase exponentially, while the positive ion velocity and negative ion density decrease exponentially. It should be noted that in the case quasi-neutrality at $x=0$, the linearized equation is a harmonic one (no right hand side term), which means that the solutions show a symmetry with the curve $\varphi=0$. 


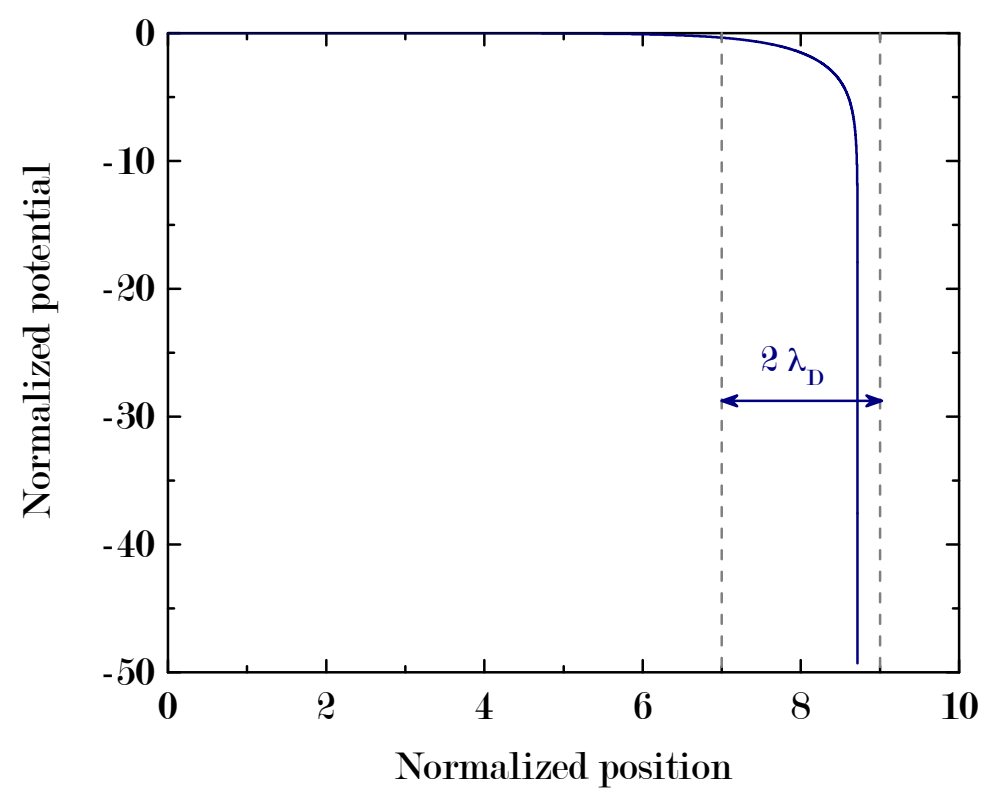

(a)

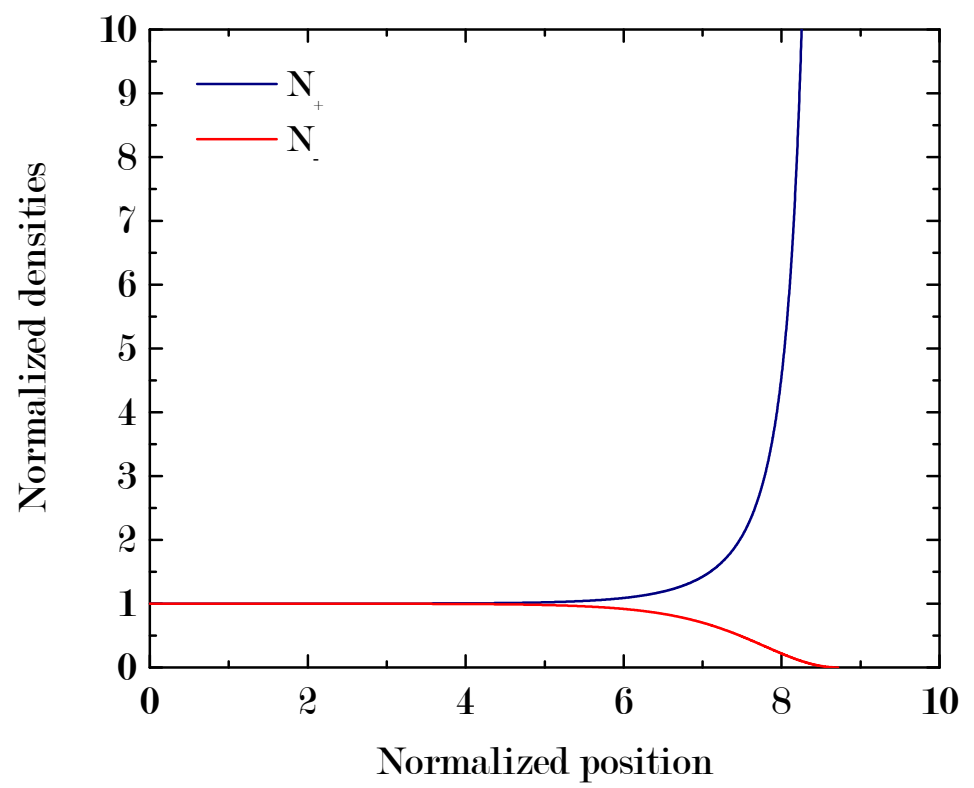

(b)

Figure 7.2: Normalized potential (a) and normalized densities (b) as functions of position in the warm-warm case. The potential is normalized to the positive ion temperature, the densities to the initial density, and the position to the Debye length. 
The characteristic length of the exponential is a modified Debye length, with quasineutrality and the ratio of temperatures parameters. It can be seen in figure 7.2 that the sheath size is roughly $2 \lambda_{D}$, and is almost insensitive to the voltage across the sheath. This is due to the exponential behavior of the potential. Since a plasma is usually assumed quasi-neutral, it means that the main parameter of the equilibrium is the ratio of temperature. As the inertia term was assumed to be negligible, the masses obviously do not play a role in the equilibrium.

In the case of the floating electrode, the positive and negative ion fluxes are equal since no current is drawn with the electrode. The bias of the electrode is called floating potential, and the potential difference $V_{p}-V_{f}$ repels a species and accelerates the other. with the Boltzmann relation, the thermal fluxes at the electrode can be written as

$$
\Gamma_{ \pm}=\frac{1}{4} n_{ \pm} \bar{v}_{ \pm} e^{ \pm\left(V_{p}-V_{f}\right) / T_{ \pm}}
$$

Equating these two fluxes and assuming quasi-neutrality $\left(n_{+}=n_{-}\right)$, the following relation is obtained

$$
\frac{\bar{v}_{-}}{\bar{v}_{+}}=e^{\left(V_{p}-V_{f}\right)\left(T_{+}^{-1}+T_{-}^{-1}\right)},
$$

and yields

$$
V_{p}-V_{f}=\frac{T_{+} T_{-}}{2\left(T_{+}+T_{-}\right)} \ln \left(\frac{m_{+}}{m_{-}} \frac{T_{-}}{T_{+}}\right) .
$$

In the case where the temperatures are equal, equation (7.18) becomes

$$
V_{p}-V_{f}=\frac{T}{4} \ln \frac{m_{+}}{m_{-}}
$$

It should be noted that when $m_{+}=m_{-}, V_{p}-V_{f}=0$.

\section{Cold-Warm Case}

The equation of the model is obtained by combining the density expressions from equations (7.6) and (7.10), and the Poisson equation (7.11)

$$
-\varepsilon_{0} \Delta \varphi=\frac{e n_{0}}{\sqrt{1-\frac{2 e}{m_{+} u_{0}^{2}} \varphi}}-e n_{0} \alpha_{d} e^{\varphi / T_{-}} .
$$

This equation is linearized as

$$
-\frac{\varepsilon_{0} T_{-}}{e n_{0}} \Delta \varphi=\left(1-\alpha_{d}\right) T_{-}+\left(\frac{e T_{-}}{m_{+} u_{0}^{2}}-\alpha_{d}\right) \varphi .
$$

Similarly to the warm-warm case, a Debye length $\lambda_{D-}^{2}=\frac{\varepsilon_{0} T_{-}}{e^{2} n_{0}}$ can be defined. One can also recognize the Mach number $\mathcal{M}=\frac{u_{0}}{u_{B+}}$ with $u_{B+}^{2}=\frac{e T_{-}}{m_{+}}$, an ion Bohm velocity. It should be noted that due to the difference in temperature between elecrons and negative ions, this ion Bohm velocity is smaller then the classical Bohm velocity in an electron-positive ion plasma. Equation (7.21) can now be rewritten as

$$
\lambda_{D-}^{2} \Delta \varphi-\left(\frac{1}{\mathcal{M}}-\alpha_{d}\right) \varphi=\left(\alpha_{d}-1\right) T_{-} .
$$


Here, the sign of the factor in front of $\varphi$ depends on the additional parameter $\mathcal{M}$, the Mach number. With $\mathcal{M}>1$, the factor is negative, which means that the solutions are diverging exponentials. With $\mathcal{M}<1$, the factor is positive, the solutions are sinusoidal oscillations. The characteristic length for either type of solution is a modified Debye length, with dependencies on the ratio of initial densities and the Mach number (ratio of the initial velocity to the ion Bohm velocity $u_{B+}^{2}=\frac{e T_{-}}{m_{+}}$. It should be noted that the problem does not depend on the ratio of initial velocities. With quasi-neutrality assumed, the equation becomes harmonic (symmetry with $\varphi=0$ ). Figure 7.3 shows the potential profile for (a) $\mathcal{M}=1.1$ (sheath solution), and (b) $\mathcal{M}=0.9$ (oscillating solution). It should be noted that the ion densities in the oscillating cold-warm are in-phase. This is due to the fact that the negative ions are in Boltzmann equilibrium.

This separation of solution types with the Mach number is similar to the discussion by Riemann [75] on the Bohm criterion for sheath formation in a classical electron-positive ion plasma. The existence of a pre-sheath is assumed between the quasi-neutral plasma with a flat potential profile and the sheath itself, accelerating the positive ions to the Bohm velocity so that the Mach number is greater than 1, ensuring that the solution is a sheath. The Bohm velocity in this case is $u_{B}=\sqrt{e T_{e} / m_{+}}$.

Assuming that there are no negative ions in the sheath, a relation between the ingoing current $J_{0}$, the potential drop across the sheath $V_{0}$ and the sheath size $s$, called the Child law [7] can be derived

$$
J_{0}=\frac{4}{9} \varepsilon_{0}\left(\frac{2 e}{m_{+}}\right)^{1 / 2} \frac{V_{0}^{3 / 2}}{s^{2}},
$$

where the drop in positive ion density as they are accelerated is taken into account (flux conservation). For a fixed current, the scaling of the sheath size as a function of the potential drop is thus

$$
s \propto V_{0}^{3 / 4} .
$$

This scaling law is useful for the grid design as the potential difference is created between two grids for a current set by the plasma. In figure 7.3 with $\mathcal{M}>1$, the sheath size is roughly $25 \lambda_{D}$ for a voltage of $60 \mathrm{~V}$ across the sheath. The fact that the sheath size depends on the voltage across the sheath can be seen as the potential gradient stays finite, as opposed to the cold-warm case in figure 7.2.

In the case of the floating potential where the mass of the negative ions is smaller than that of the positive ions, the diffusion of negative ions is faster than that of positive ions. This means that as negative ions escape the plasma, the surrounding walls charge up negatively until the electric field near the wall (potential drop in sheath) is sufficient to confine the negative ions and accelerate the positive ions outward, allowing the plasma to remain quasi-neutral. This is the reason why the plasma potential in an electronpositive ion plasma is always higher than the potential at the wall (no magnetic field). The potential difference between the plasma $V_{p}$ and the wall $V_{f}$ compensates for the fact that the negative ion current is greater than the positive ion current. With the assumption of a thermal flux at the electrode for the negative ions

$$
\Gamma_{-}=\frac{1}{4} n_{-} \bar{v}_{-} e^{-\left(V_{p}-V_{f}\right) / T_{-}},
$$

and a Bohm flux for the positive ions

$$
\Gamma_{+}=n_{+} u_{B},
$$




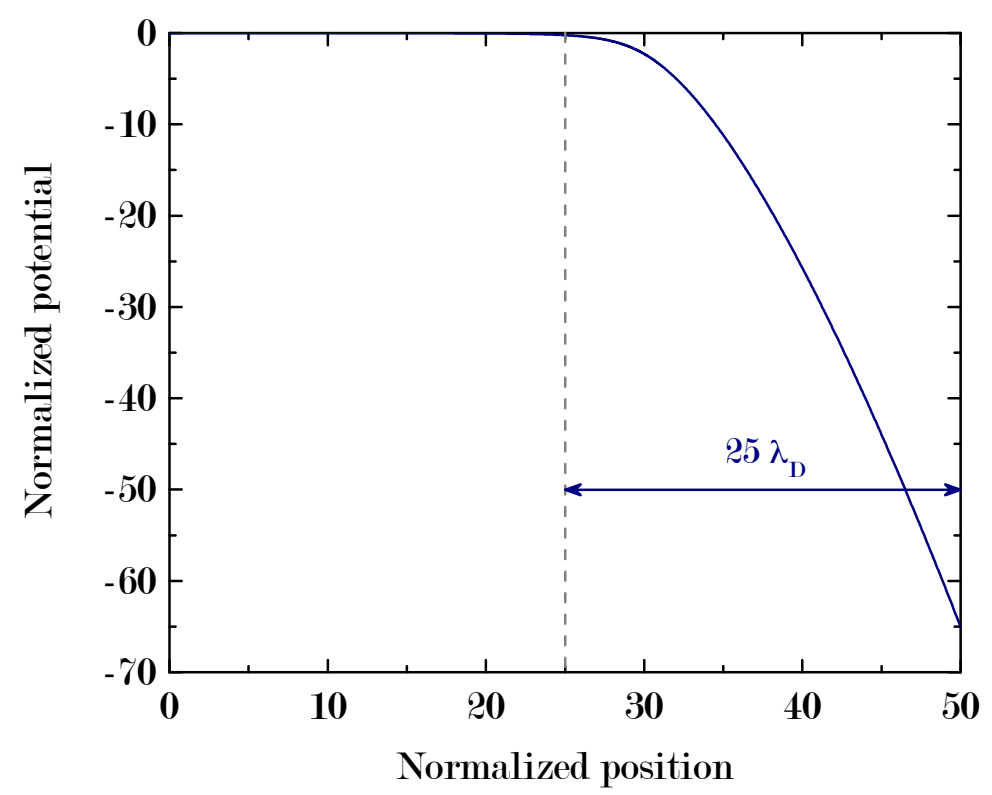

(a) Cold-warm with $\mathcal{M}=1.1$

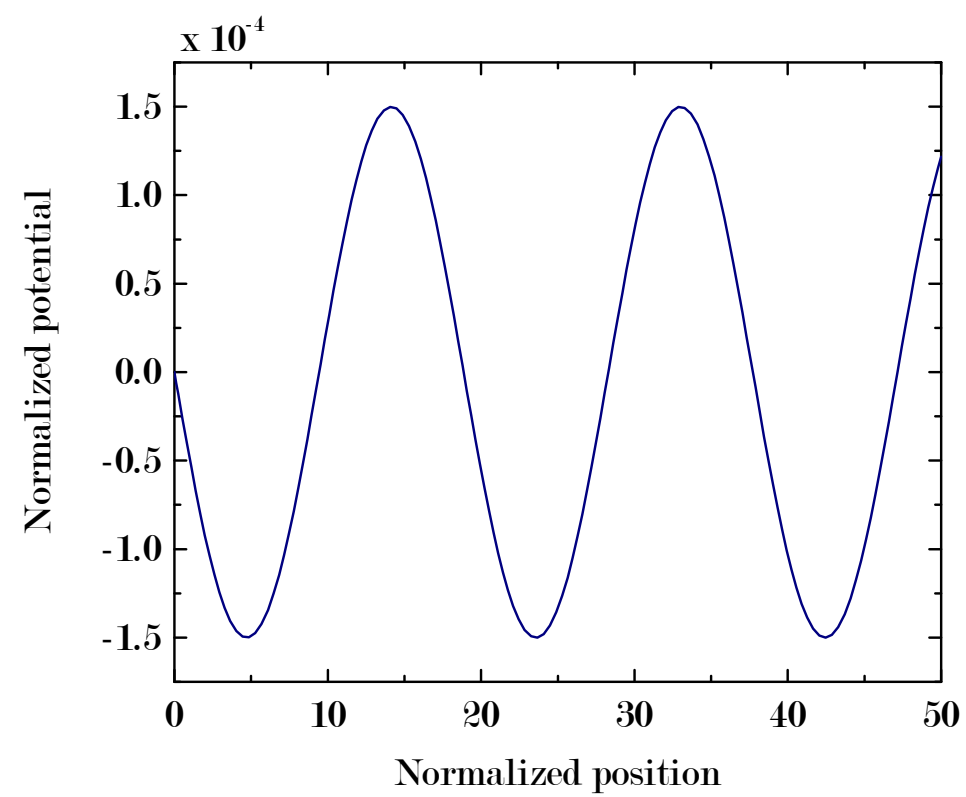

(b) Cold-warm with $\mathcal{M}=0.9$

Figure 7.3: Normalized potential as a function of position for the cold-warm case, with $\mathcal{M}=1.1$ (a) and $\mathcal{M}=0.9$ (b). $\mathcal{M}=u_{0} / u_{B+}$ is defined as the Mach number. The potential is normalized to the negative ion temperature and the position to the Debye length. 
the potential difference can be calculated as

$$
V_{p}-V_{f}=\frac{T_{-}}{2} \ln \frac{m_{+}}{2 \pi m_{-}}
$$

\subsubsection{Ion-Ion Plasma}

Two cases were considered for the modeling of an ion-ion plasma, using two assumptions (warm and cold). As an ion-ion plasma should be symmetrical (same masses and temperatures), the cold-warm case seems an unlikely candidate. The expression of the floating potential shows that it cannot be used to describe a symmetrical ion-ion plasma since it gives a positive value

$$
V_{p}-V_{f}=-\frac{T}{2} \ln 2 \pi
$$

instead of the correct value $V_{f}-V_{p}=0$ as both ion currents have the same value, without the need for a retarding field. The floating potential in the warm-warm case (7.19), however, gives the correct value $V_{p}-V_{f}=0$.

In the following, PIC simulations and a kinetic theory are developed to describe the transition between the two extreme cases.

\subsection{PIC Simulations}

The PIC simulations were established by Albert Meige, and resulted in a publication [76] (see end of the thesis). An ion-ion plasma is simulated to understand the potential profile, as well as density and velocity profiles, in the case of biased electrodes.

\subsubsection{Description of the PIC Simulations}

A self-consistent 1D particle-in-cell (PIC) simulation (no a priori assumptions on the energy distribution) was developed in the case of an ion-ion plasma: a positive ion species and a negative ion species [76]. This PIC simulation is based on the scheme from [77] and [78]. No source terms (whether creation or loss) are considered inside the simulation box, which means no ionization, attachment or recombination. Also, the plasma is assumed collisionless. Particles are lost through absorbing boundaries, which are directcurrent polarized electrodes: the left electrode is polarized to $+V_{0}$ and the right electrode to $-V_{0}$, giving a total potential difference of $2 V_{0}$. In order to compensate those losses, ions are introduced in the simulation box over its entire length with a uniform probability profile. The initial velocity of each ion is randomly taken from a Maxwellian distribution.

The length of the simulation box is set to $1 \mathrm{~cm}$. The plasma is symmetrical with $m_{+}=m_{-}=40$ a.m.u., $T_{+}=T_{-}=26 \mathrm{mV}(300 \mathrm{~K})$ and singly charged ions. Simulations are 10 to $100 \mathrm{~ms}$ long, with time steps between $10^{-10}$ and $10^{-9} \mathrm{~s}$. Simulations are stopped when a steady-state is reached. Up to $2 \times 10^{6}$ macro-particles per species were used for a central density of $\sim 10^{16} \mathrm{~m}^{-3}$. 


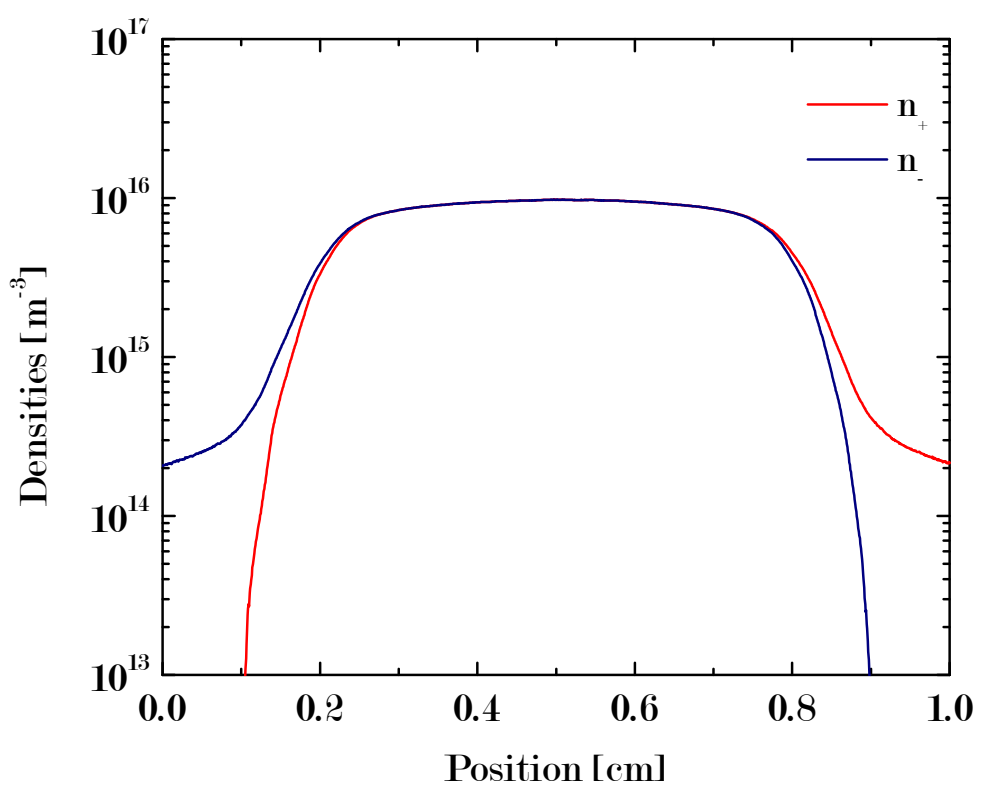

Figure 7.4: Averaged densities as a function of position, with the positive ion density in red and the negative ion density in blue. The potential difference is set to $50 \mathrm{~V}$.

\subsubsection{Results}

\section{Density and Potential Profiles}

The results shown in figures 7.4 and 7.5 were obtained with a direct-current potential difference of $50 \mathrm{~V}$, i.e. the left electrode is polarized to $-25 \mathrm{~V}$ and the right electrode to $+25 \mathrm{~V}$. Contrary to a classical electron-ion plasma where the plasma potential is higher than either electrode, the plasma potential for an ion-ion plasma is seen to be at an intermediate value between the electrode potentials, $V_{p}=0$. Although the quasi-neutral region looks flat in comparison to the sheaths, there is a very small potential drop of the order of the ion temperature $T_{ \pm} / 2$. Similarly to an electron-ion plasma, this small potential drop is the equivalent of a pre-sheath, accelerating the ions to an ion Bohm velocity.

A sheath is created in front of each electrode after a few microseconds, with opposite potential differences. The fact that the ion-ion plasma was assumed to be symmetrical (masses and temperatures) can be seen in the symmetrical profile for the densities and the potential. A few cases were run with different masses and/or different temperatures with a shift in plasma potential as a result. The potential difference of the sheath corresponding to the lighter species is reduced while the other sheath's potential difference is increased accordingly to maintain a total potential difference of $2 V_{0}$. In the same way, the potential difference for the species with the smaller temperature is reduced while the one for the other sheath is increased.

\section{Velocity Profiles}

Figure 7.6 shows the averaged velocity of all particles at a given $x$ as a function of position. It was arbitrarily decided that the sheath begins as $\Delta n / n$ reaches $10 \%$. Given the steep profiles of the densities shown in figure 7.4, choosing this value around 5-10 does not 


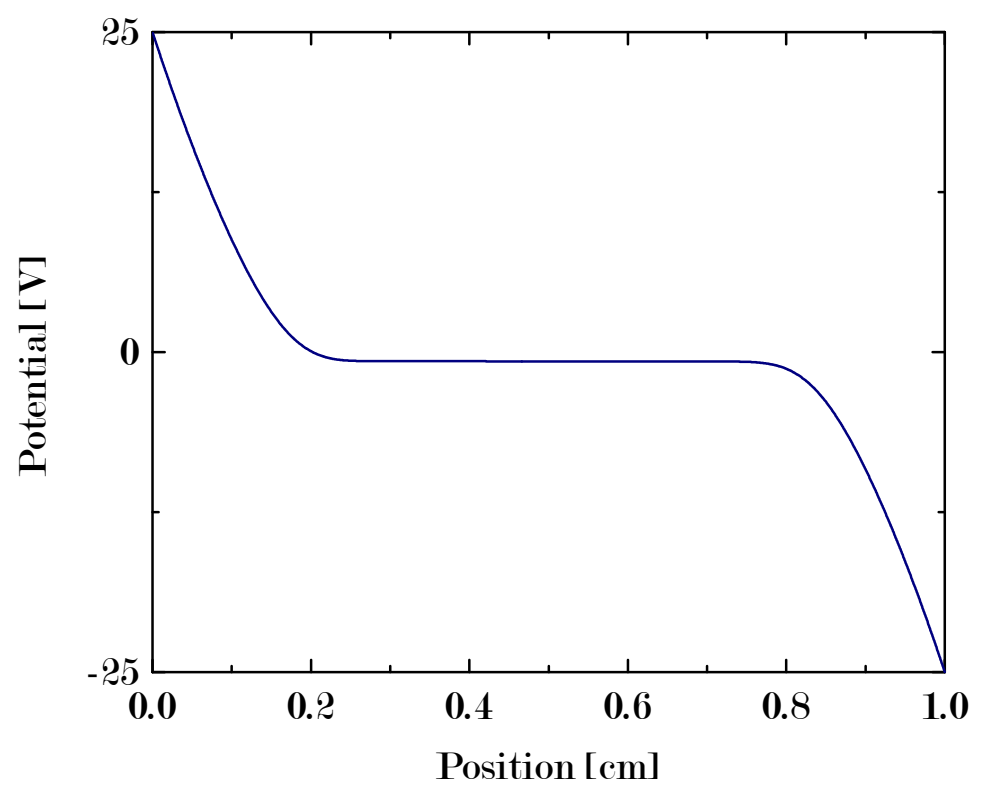

Figure 7.5: Potential as a function of position. The potential difference is set to $50 \mathrm{~V}$.

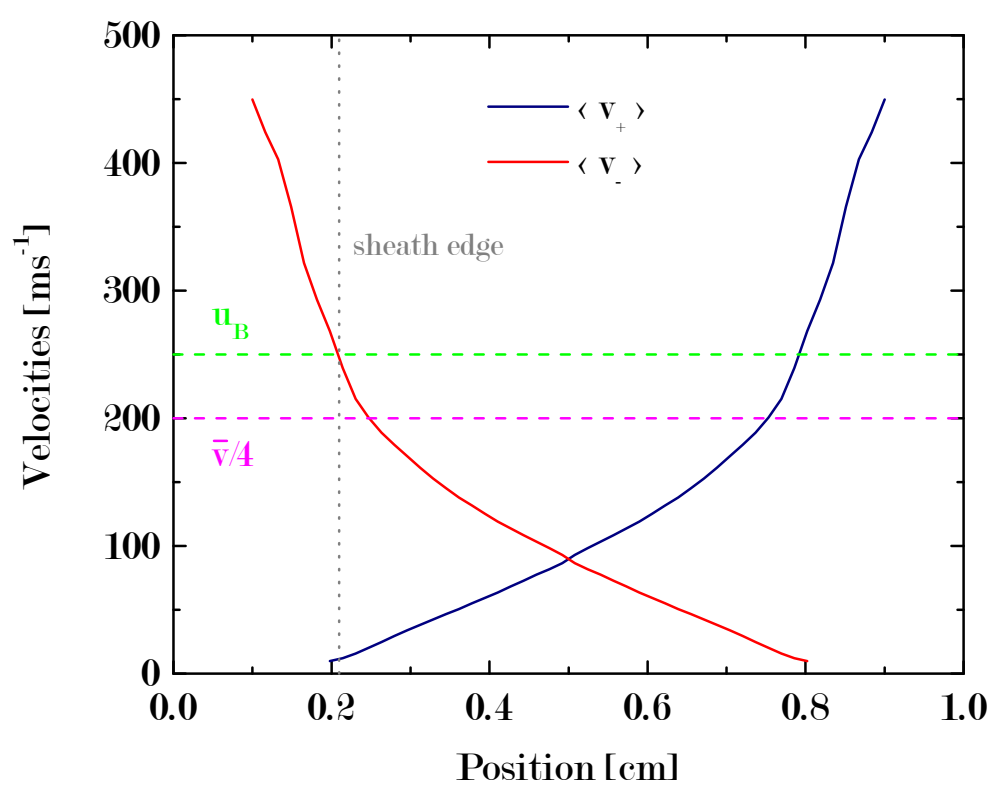

Figure 7.6: Absolute averaged velocities for positive ions (blue) and negative ions (red) as a function of position. The sheath edge is shown as a gray dotted line, the thermal velocity as a purple dashed line, and the Bohm velocity as a green dashed line. 


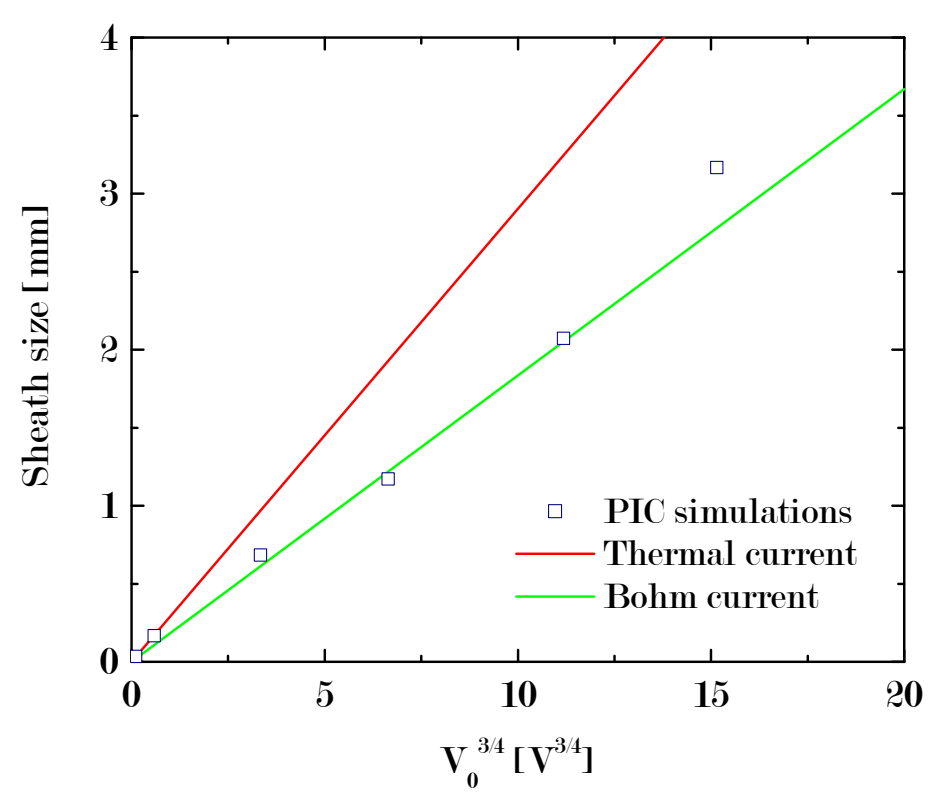

Figure 7.7: Sheath size as a function of potential difference across the sheath to the power of $\frac{3}{4}$ for the PIC simulations, the Child law with a thermal current, and the Child law with a Bohm current.

change the results. It can be seen in figure 7.6 that the velocity of the accelerated species as it enters the sheath is not the thermal velocity $(\bar{v})$, but a higher velocity that is an ion Bohm velocity. For a range of simulation parameters, the ion velocity at the sheath entry was compared to the expression of the Bohm velocity adapted to an ion-ion plasma, $u_{B}=\sqrt{e T_{ \pm} / m_{\mp}}$. In each case, the agreement was excellent, showing that there is indeed a Bohm condition at the sheath edge in an ion-ion plasma.

The effect of the small potential drop across the quasi-neutral region, mentioned previously, can be seen in the fact that both ion species are accelerated from the sheath where they are reflected (left electrode for the positive ions), until the sheath where they are accelerated (right electrode for the positive ions). Once in the sheath, the ions are accelerated more rapidly because of the bigger potential drop.

The velocities as a function of position are symmetrical around the center value $x=$ $0.5 \mathrm{~cm}$, which is due to the symmetry of the ion-ion plasma considered.

\section{Child Law}

The Child law (7.23) can be used to determine the sheath size $s$ as a function of the potential difference across the sheath $V_{0}$, with the scaling $s \propto V_{0}^{3 / 4}$. The factor of proportionality depends on the ion current $J_{0}$. Figure 7.7 shows the scaling law in the PIC simulations compared to the Child law scalings using two different currents: the thermal current $J_{0}=\frac{1}{4} e n \bar{v}$ and the Bohm current $J_{0}=e n \sqrt{e T_{ \pm} / m_{\mp}}$.

The first interesting feature of this figure, is the fact that the sheath size does indeed follow the Child law scaling. This can be seen as the sheath size being a linear function of $V_{0}^{3 / 4}$. Moreover, the current for the Child law is the Bohm current and not the thermal current, confirming what was seen with the velocity profiles as a function of position.

The Child law is derived with the assumption that the accelerated ions are inertia dominated. The fact that it applies to an ion-ion plasma shows that the cold-warm 
approximation presented previously is a better approximation than the warm-warm approximation to describe high voltage sheaths.

\subsubsection{PIC Limitations}

The PIC simulations consider a pure ion-ion plasma without any volume creation or loss. For the plasma to be sustained, a uniform ion-ion plasma is continuously introduced over the whole length, with a velocity distribution given by a Maxwellian at a given temperature. As a result, an ion-ion plasma is introduced in the sheath regions, in front of each electrode, with a non-negligible electric field. Both ion species are accelerated, one toward the electrode, one toward the quasi-neutral plasma. The quasi-neutral region is thus a combination of an ion-ion plasma with a temperature that can be controlled and two ion beams going in opposite directions from one sheath to the other. The resulting temperature of the ion-ion plasma is higher than that of the ion-ion plasma introduced. The presence of ion beams could also modify the equilibrium of the ion-ion plasma in respect to the polarized electrodes.

\subsection{Kinetic Model}

It was shown by the PIC simulations that the response of an ion-ion plasma to polarized electrodes is a screening effect (creation of sheaths at the electrodes), where the velocity of ions must reach a Bohm velocity. A kinetic model was developed to understand the evolution of the ion species as they enter the sheath: depending on the charge and velocity of the ion, it is accelerated, slowed down or reflected. The high and low voltage sheaths are investigated.

\subsubsection{Assumptions}

A one-dimensional plasma composed of two ion species is considered in the sheath region, which is assumed to have a length $L$. Moreover, the sheath is assumed to be monotonic, i.e. the potential is a monotonic function of position. It should be noted that the positive ions are now the reflected species while the negative ions are accelerated in the sheath. The potential difference is set to $\varphi_{L}$ with the following boundary conditions:

$$
\varphi(0)=0, \quad \varphi(L)=\varphi_{L}>0 .
$$

Quasi-neutrality is assumed at the sheath edge $x=0$, unless specified otherwise

$$
n_{+}(0)=n_{-}(0)
$$

The schematic of the model is shown in figure 7.8. Although the plasma region is shown, the model only deals with the sheath ( $x=0$ corresponds to the sheath edge).

To solve this problem, the Vlasov equations for each species

$$
\partial_{t} f_{ \pm}+\partial_{x}\left(v f_{ \pm}\right)+\partial_{v}\left(a f_{ \pm}\right)=0
$$

coupled to the Poisson equation are used.

To simplify this equation, several assumptions are made:

- steady-state: $\partial_{t}$. $=0$, 


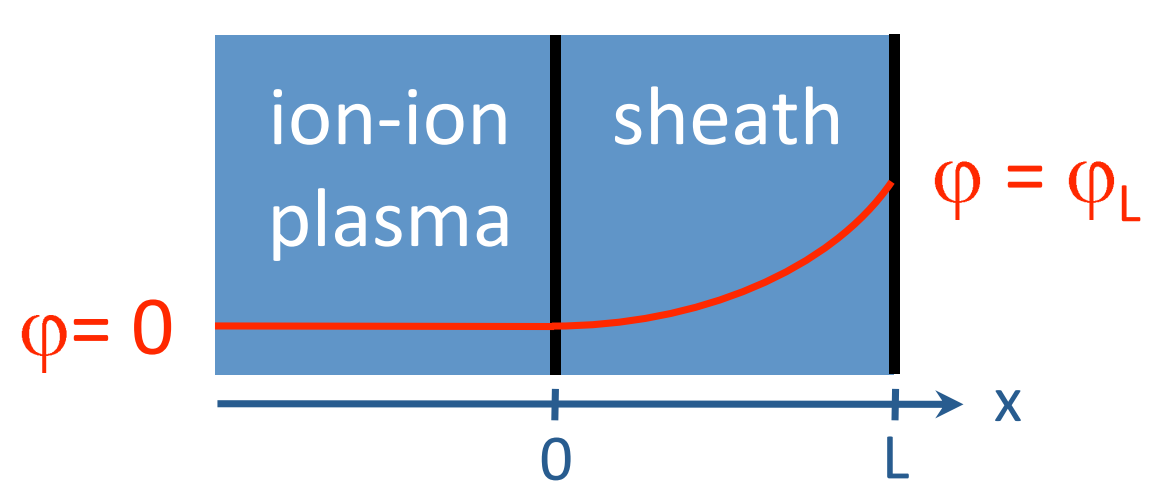

Figure 7.8: Schematic of the kinetic model.

- acceleration and velocity are independent: $\partial_{v} a=0$,

resulting in

$$
v \partial_{x} f_{ \pm}+a \partial_{v} f_{ \pm}=0
$$

The only force considered is the electric force, giving the expression of the acceleration

$$
a= \pm \frac{e E}{m_{ \pm}},
$$

with $e$ the charge of the electron, $E$ the electric field and $m_{ \pm}$the masses of the ions. The Vlasov equation for the problem is obtained by combining (7.32) and (7.33)

$$
\partial_{x} f_{ \pm} \pm \frac{e E}{m_{ \pm} v} \partial_{v} f_{ \pm}=0
$$

Since the electric field is a function of position, any function $g\left(\beta_{1}(v)+\beta_{2}(x)\right)$, with well chosen functions $\beta_{1}$ and $\beta_{2}$, is a solution of the Vlasov equation. For instance, the functions $\beta_{1}(v)=\frac{1}{2} v^{2}$ and $\beta_{2}(x)= \pm \frac{e \varphi}{m_{ \pm}}$make a solution. Using the dimension of velocity, any function $g$ of the following parameter is solution

$$
g\left(\sqrt{v^{2} \pm \frac{2 e \varphi}{m_{ \pm}}}\right)
$$

This solution can be interpreted as a balance between kinetic energy and potential energy, the total energy being conserved. The distribution functions solutions to the Vlasov equations, therefore, can be written as

$$
f_{ \pm}(x, v)=f_{ \pm}\left(0, \sqrt{v^{2} \pm \frac{2 e \varphi}{m_{ \pm}}}\right)=f_{ \pm}^{0}\left(\sqrt{v^{2} \pm \frac{2 e \varphi}{m_{ \pm}}}\right)
$$

with $f_{ \pm}^{0}$ the distribution functions at the sheath edge $(x=0)$ as functions of velocity only.

The Poisson equation

$$
\varepsilon_{0} \partial_{x} E=e\left(n_{+}-n_{-}\right),
$$

and the $n$ 's the densities of the ion species, combined with the relation between electric field and potential $\partial_{x} \varphi=-E$ and the definition of the densities from the distribution functions yields

$$
-\varepsilon_{0} \partial_{x}^{2} \varphi=e\left(\int_{-\infty}^{+\infty} f_{+}(x, v) d v-\int_{-\infty}^{+\infty} f_{-}(x, v) d v\right) .
$$




\subsubsection{Initial Distribution Function Requirements}

The distribution functions $f_{ \pm}^{0}$ are assumed to be Maxwellian, but due to the behavior of ions in the sheath, these cannot be full Maxwellians. Since $\varphi_{L}>0$, negative ions are accelerated while positive ions are either slowed down or reflected. The limiting velocity for a positive ion deciding whether it is reflected or not comes from equating the kinetic energy and the potential energy

$$
\frac{1}{2} m_{+} v_{r}^{2}=e \varphi_{L}
$$

giving the expression of the limit velocity $v_{r}$

$$
v_{r}=\sqrt{\frac{2 e \varphi_{L}}{m_{+}}}
$$

The range of velocities for the positive ions entering the sheath does not have limitations. The distribution function considered for these ions is thus a full Maxwellian truncated at $-v_{r}$ with all values below zero

$$
f_{+}^{0}\left(v_{0}\right)=A_{+} \exp \left(-\frac{m_{+} v_{0}^{2}}{2 e T_{+}}\right) \theta\left(v_{0}+\sqrt{\frac{2 e \varphi_{L}}{m_{+}}}\right)
$$

with $A_{+}$a constant to be determined from the positive ion density at $x=0$ and $\theta$ the Heaviside step function.

Also, to be consistent with the PIC simulations that showed a minimum velocity for accelerated ions entering the sheath, the distribution function considered for the negative ions is a $v>0$ half-Maxwellian shifted to the velocity $v_{\text {inj. }}$. The shift to $v_{\text {inj }}$ represents a crude model for the pre-acceleration in a pre-sheath

$$
f_{-}^{0}\left(v_{0}\right)=A_{-} \exp \left(-\frac{m_{-}\left(v_{0}-v_{\text {inj }}\right)^{2}}{2 e T_{-}}\right) \theta\left(v_{0}-v_{\text {inj }}\right)
$$

with $A_{-}$a constant to be determined from the negative ion density at $x=0$.

Figure 7.9 shows initial distribution functions.

With a zero initial electric field, all variables stay constant. The sheath, therefore, needs to be triggered, which is done with a small initial electric field. This initial electric field has to be negative as the desired potential gradient is positive (positive ions are reflected and negative ions accelerated). When the equation of the model is integrated, the initial electric field is set to $E_{0}=-5 \times 10^{-5} \mathrm{Vm}^{-1}$, unless stated otherwise. It was verified that the model results are independent of the exact value of $E_{0}$, as long as it is very small. 


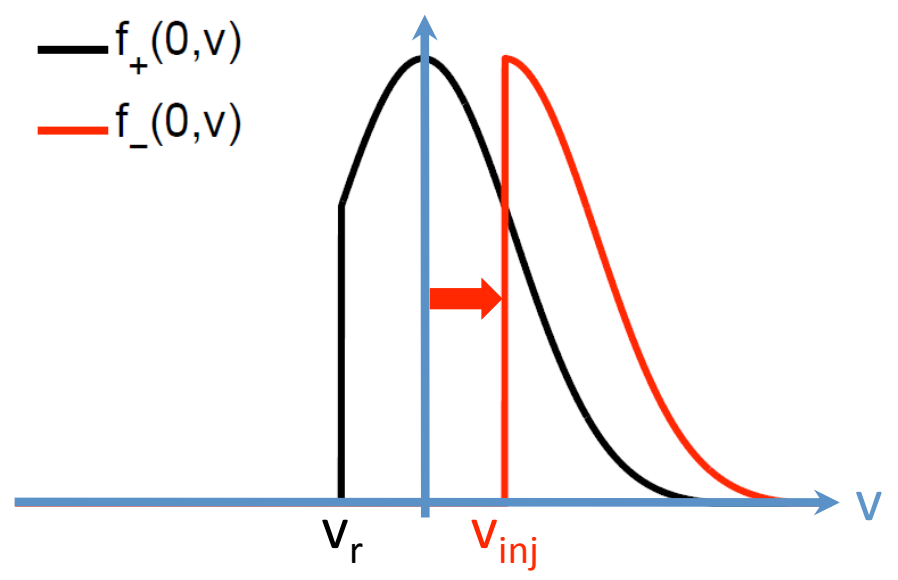

Figure 7.9: Initial distribution functions (b) for both ion species.

\subsubsection{Equation of the Model}

With the expressions of the initial distribution functions (7.41) and (7.42), the distribution functions as functions of position and velocities can be obtained by using (7.36)

$$
\begin{aligned}
& f_{+}(x, v)=A_{+} \exp \left(\frac{m_{+} v^{2}}{2 e T_{+}}\right) \exp \left(-\frac{\varphi(x)}{T_{+}}\right) \theta\left(v+\sqrt{\frac{2 e\left(\varphi_{L}-\varphi(x)\right)}{m_{+}}}\right), \\
& f_{-}(x, v)=A_{-} \exp \left[-\frac{m_{-}\left(\sqrt{v^{2}-\frac{2 e \varphi(x)}{m_{-}}}-v_{\mathrm{inj}}\right)^{2}}{2 e T_{-}}\right] \theta\left(v-\sqrt{v_{\mathrm{inj}}^{2}+\frac{2 e \varphi(x)}{m_{-}}}\right) .
\end{aligned}
$$

The expressions for the parameters of the Heaviside functions come from the conservation of energy. For the positive ions, the limit velocity as a function of position is decreasing as the potential increases

$$
\frac{1}{2} m_{+} v_{r}^{2}(x)+e \varphi(x)=e \varphi_{L} \quad \Rightarrow \quad v_{r}(x)=\sqrt{\frac{2 e\left(\varphi_{L}-\varphi(x)\right)}{m_{+}}} .
$$

For the negative ions, the minimum velocity is increased from $v_{\text {inj }}$ as the potential increases

$$
\frac{1}{2} m_{-} v_{\mathrm{min}}^{2}-e \varphi(x)=\frac{1}{2} m_{-} v_{\mathrm{inj}}^{2} \Rightarrow v_{\min }=\sqrt{v_{\mathrm{inj}}^{2}+\frac{2 e \varphi(x)}{m_{-}}} .
$$

The densities can then be calculated with the definition

$$
n_{ \pm}(x)=\int_{-\infty}^{+\infty} f_{ \pm}(x, v) d v
$$

and the use of $\operatorname{erf}(x)=\frac{2}{\sqrt{\pi}} \int_{0}^{x} e^{-t^{2}} d t$. The positive ion density can be integrated as

$$
n_{+}(x)=\sqrt{\frac{\pi e T_{+}}{2 m_{+}}} A_{+} \exp \left(\frac{-\varphi(x)}{T_{+}}\right)\left[1+\operatorname{erf}\left(\sqrt{\frac{\varphi_{L}-\varphi(x)}{T_{+}}}\right)\right],
$$


while the negative ion density can only be expressed as an integral

$$
n_{-}(x)=\int_{\sqrt{v_{\mathrm{inj}}^{2}+\frac{2 e \varphi(x)}{m_{-}}}}^{+\infty} A_{-} \exp \left[-\frac{m_{-}\left(\sqrt{v^{2}-\frac{2 e \varphi(x)}{m_{-}}}-v_{\mathrm{inj}}\right)^{2}}{2 e T_{-}}\right] d v
$$

The initial ion densities can be calculated as

$$
\begin{aligned}
& n_{+}(0)=\sqrt{\frac{\pi e T_{+}}{2 m_{+}}} A_{+}\left[1+\operatorname{erf}\left(\sqrt{\varphi_{L} / T_{+}}\right)\right], \\
& n_{-}(0)=\sqrt{\frac{\pi e T_{-}}{2 m_{-}}} A_{-} .
\end{aligned}
$$

Quasi-neutrality is given by $\alpha=1$, when ion densities are equal. However, it should be noted that because of the different distribution functions, not all ions enter the sheath: the entire negative ion population enters the sheath and is accelerated $\left(n_{0-}\right)$, but the entering positive ion population $\left(n_{0+} /\left(1+\operatorname{erf} \sqrt{\varphi_{L} / T_{+}}\right)\right)$is divided between

- a reflected part: $\left(n_{0+} \operatorname{erf} \sqrt{\varphi_{L} / T_{+}}\right) /\left(1+\operatorname{erf} \sqrt{\varphi_{L} / T_{+}}\right)$

- and a part with a high enough velocity to overcome the potential barrier: $n_{0+}\left(1-\operatorname{erf} \sqrt{\varphi_{L} / T_{+}}\right) /\left(1+\operatorname{erf} \sqrt{\varphi_{L} / T_{+}}\right)$.

The variables are normalized as $N_{+}=n_{+} / n_{0-}, N_{-}=n_{-} / n_{0-}, V_{-}=v_{-} / v_{0_{-}}, \Phi=\varphi / \varphi_{0}$ with

$$
\left[\begin{array}{rl}
n_{0+} & =\sqrt{\frac{\pi e T_{+}}{2 m_{+}}} A_{+}\left[1+\operatorname{erf}\left(\sqrt{\varphi_{L} / T_{+}}\right)\right] \\
n_{0-} & =\sqrt{\frac{\pi e T_{-}}{2 m_{-}}} A_{-} \\
v_{0-} & =\sqrt{\frac{2 e T_{-}}{m_{-}}} \\
\varphi_{0} & =T_{-} \\
\alpha & =\frac{n_{0+}}{n_{0-}} \\
\gamma & =\frac{T_{-}}{T_{+}} \\
\delta & =\frac{m_{-}}{m_{+}} \\
\lambda_{D}^{2} & =\frac{\varepsilon_{0} T_{-}}{e n_{0-}}
\end{array}\right.
$$

and can be rewritten as

$$
\begin{aligned}
& N_{+}(x)=\alpha e^{-\gamma \Phi(x)} \frac{1+\operatorname{erf} \sqrt{\gamma\left(\Phi_{L}-\Phi(x)\right)}}{1+\operatorname{erf} \sqrt{\gamma \Phi_{L}}} \\
& N_{-}(x)=\frac{2}{\sqrt{\pi}} \int_{\sqrt{V_{\mathrm{inj}}^{2}+\Phi(x)}}^{+\infty} \exp \left[-\left(\sqrt{V^{2}-\Phi(x)}-V_{\mathrm{inj}}\right)^{2}\right] d V,
\end{aligned}
$$


with $\Phi_{L}=\varphi_{L} / \varphi_{0}$ and $V_{\text {inj }}=v_{\text {inj }} / v_{0-}$.

In order to normalize the Poisson equation (7.38), two additional parameters are needed

$$
\left[\begin{array}{c}
X=\frac{x}{L_{0}} \\
\epsilon=\frac{\lambda_{D}}{L_{0}}
\end{array},\right.
$$

yielding the normalized Poisson equation $-\epsilon^{2} d_{X}^{2} \Phi=N_{+}(X)-N_{-}(X) . L_{0}$ is a normalization length and does not hold any physical meaning. The equation of the model, therefore, is

$$
\begin{aligned}
\epsilon^{2} d_{X}^{2} \Phi=\frac{2}{\sqrt{\pi}} \int_{\sqrt{V_{\mathrm{inj}}^{2}+\Phi(x)}}^{+\infty} \exp \left[-\left(\sqrt{V^{2}-\Phi(x)}-V_{\mathrm{inj}}\right)^{2}\right] d V & \\
& -\alpha e^{-\gamma \Phi(x)} \frac{1+\operatorname{erf} \sqrt{\gamma\left(\Phi_{L}-\Phi(x)\right)}}{1+\operatorname{erf} \sqrt{\gamma \Phi_{L}}}
\end{aligned}
$$

To solve the integral with an infinite boundary, a modified integration function of Matlab developped by Shampine [79] is used. The main output of an integration is the length of the simulation $L$. Since the function erf is only defined numerically for real values and not complex ones, the equation is only valid for $\Phi \leq \Phi_{L}$, which can cause some numerical problems as $\Phi$ reaches $\Phi_{L}$. For instance, the last integration increment $\Delta X_{n-1}$ might be bigger than the distance between $X_{n-1}$, the next to last step, and $L$, resulting in the equation being calculated with $X_{n}>L$, i.e. $\Phi\left(X_{n}\right)>\Phi_{L}$.

Once equation (7.56) is solved for the potential, the densities are obtained from (7.53) and (7.54). The fluid velocity of the negative ion species can be calculated by using the definition

$$
<v_{-}(x)>=\frac{\int_{-\infty}^{+\infty} v f_{-}(x, v) d v}{\int_{-\infty}^{+\infty} f_{-}(x, v) d v}
$$

which becomes with the normalization from (7.52)

$$
<V_{-}(X)>=\frac{1}{N_{-}(X)} \frac{2}{\sqrt{\pi}} \int_{\sqrt{V_{\mathrm{inj}}^{2}+\Phi(X)}}^{+\infty} V \exp \left[-\left(\sqrt{V^{2}-\Phi(X)}-V_{\mathrm{inj}}\right)^{2}\right] d V
$$

\subsubsection{Bohm Criterion}

Since the PIC simulations showed the existence of an ion Bohm criterion for the sheath in an ion-ion plasma, a formulation of such a criterion in this kinetic model is needed. The Bohm criterion ensures that the sheath at the edge of a quasi-neutral plasma is monotonic, and can be expressed as a condition on the accelerated species (positive ions in an electron-ion plasma for instance) or on the derivative of the densities of both species in respect to the potential, the latter case requiring a monotonic potential as function of position for the potential to be used as coordinates. Following Chen [80], and taking into account that the accelerated species is the negative ion species, the Bohm criterion can be written as

$$
B=\left.\frac{d N_{-}}{d \Phi}\right|_{\Phi=0}-\left.\frac{d N_{+}}{d \Phi}\right|_{\Phi=0} \geq 0
$$


with $\Phi(X=0)=0$. The derivative of the positive ion density is calculated from (7.53)

$$
\frac{d N_{+}}{d \Phi}=\frac{-\alpha \gamma e^{-\gamma \Phi}}{1+\operatorname{erf} \sqrt{\gamma \Phi_{L}}}\left[1+\operatorname{erf}\left(\sqrt{\gamma\left(\Phi_{L}-\Phi\right)}\right)-\frac{1}{\sqrt{\pi}} \frac{e^{-\gamma\left(\Phi_{L}-\Phi\right)}}{\sqrt{\gamma \Phi_{L}}}\right]
$$

and the derivative of the negative ion density from (7.54) and the general formula for $G(z)=\int_{a(z)}^{b(z)} g(x, z) d x$

$$
\frac{d G(z)}{d z}=\int_{a(z)}^{b(z)} \frac{\partial g(x, z)}{\partial z} d x+g(b(z), z) \frac{d b(z)}{d z}-g(a(z), z) \frac{d a(z)}{d z}
$$

resulting in

$$
\frac{d N_{-}}{d \Phi}=\frac{-1}{\sqrt{\pi}} \frac{1}{\sqrt{V_{\mathrm{inj}}^{2}+\Phi}}+\frac{2}{\sqrt{\pi}} \int_{\sqrt{V_{\mathrm{inj}}^{2}+\Phi}}^{+\infty} \frac{\sqrt{V^{2}-\Phi}-V_{\mathrm{inj}}}{\sqrt{V^{2}-\Phi}} \exp \left[-\left(\sqrt{V^{2}-\Phi}-V_{\mathrm{inj}}\right)^{2}\right] d V
$$

Both terms of the Bohm criterion (7.59) can now be calculated

$$
\begin{aligned}
& \left.\frac{d N_{+}}{d \Phi}\right|_{\Phi=0}=-\alpha \gamma-\alpha \sqrt{\frac{\gamma}{\pi \Phi_{L}}} \frac{e^{-\gamma \Phi_{L}}}{1+\operatorname{erf} \sqrt{\gamma \Phi_{L}}}, \\
& \left.\frac{d N_{-}}{d \Phi}\right|_{\Phi=0}=\frac{-1}{\sqrt{\pi} V_{\mathrm{inj}}}+\frac{2}{\sqrt{\pi}} \int_{V_{\mathrm{inj}}}^{+\infty} \frac{V-V_{\mathrm{inj}}}{V} e^{-\left(V-V_{\mathrm{inj}}\right)^{2}} d V .
\end{aligned}
$$

Using the functions $\operatorname{erfi}(z)=-i \operatorname{erf}(i z)$ and $\operatorname{Ei}(z)=-\int_{-z}^{+\infty} \frac{1}{t} e^{-t} d t$, the negative ion term can be rewritten as

$$
\left.\frac{d N_{-}}{d \Phi}\right|_{\Phi=0}=\frac{-1}{\sqrt{\pi} V_{\mathrm{inj}}}+1+\frac{V_{\mathrm{inj}}}{\sqrt{\pi}} e^{-V_{\mathrm{inj}}^{2}}\left[\operatorname{Ei}\left(V_{\mathrm{inj}}^{2}\right)-\pi \operatorname{erfi}\left(V_{\mathrm{inj}}\right)\right] .
$$

The expression of $B$ is thus

$$
B=1+\alpha \gamma-\frac{1}{\sqrt{\pi} V_{\mathrm{inj}}}+\alpha \sqrt{\frac{\gamma}{\pi \Phi_{L}}} \frac{e^{-\gamma \Phi_{L}}}{1+\operatorname{erf} \sqrt{\gamma \Phi_{L}}}+\frac{V_{\mathrm{inj}}}{\sqrt{\pi}} e^{-V_{\mathrm{inj}}^{2}}\left[\operatorname{Ei}\left(V_{\mathrm{inj}}^{2}\right)-\pi \operatorname{erfi}\left(V_{\mathrm{inj}}\right)\right] .
$$

Similarly to the equation of the model (7.56), the boundary condition $\Phi_{L}$ appears in the expression of the Bohm criterion. However, it can be seen that, whatever the value of $\Phi_{L}$ is, for the Bohm criterion to be fulfilled, i.e. $B \geq 0$, the injection velocity $V_{\text {inj }}$ cannot be zero because of the third term, $-\left(\sqrt{\pi} V_{\mathrm{inj}}\right)^{-1}$. A necessary condition for a sheath, therefore, is $V_{\text {inj }} \neq 0$. For high potentials, i.e. $\Phi_{L}>3$ (term dependent on $\Phi_{L}$ negligible), the Bohm criterion is no longer a function of the end potential and can be written as

$$
B_{\infty}=1+\alpha \gamma-\frac{1}{\sqrt{\pi} V_{\text {inj }}}+\frac{V_{\text {inj }}}{\sqrt{\pi}} e^{-V_{\text {inj }}^{2}}\left[\operatorname{Ei}\left(V_{\text {inj }}^{2}\right)-\pi \operatorname{erfi}\left(V_{\text {inj }}\right)\right] .
$$

For a symmetrical plasma, i.e. $\alpha=\gamma=1$, the minimum value of $V_{\text {inj }}$ can be calculated independently of the density and the temperature: $\min V_{\text {inj }}=0.372$. With $n_{0}=10^{16} \mathrm{~m}^{-3}$ and $T=26 \mathrm{mV}$, the dimensioned value is: $\min v_{\text {inj }}=131 \mathrm{~m} \cdot \mathrm{s}^{-1}$. It was verified numerically that this criterion does indeed predict whether a monotonic sheath is possible or not. The value of the initial normalized velocity is obtained from (7.58)

$$
V_{-}(0)=V_{\text {inj }}+\frac{1}{\sqrt{\pi}}
$$


which gives $v_{0-}=330 \mathrm{~ms}^{-1}$ for $\alpha=\gamma=1, n_{0}=10^{16} \mathrm{~m}^{-3}$ and $T=26 \mathrm{mV}$. Comparing to the Bohm velocity found in the PIC simulations for the same conditions $\left(250 \mathrm{~ms}^{-1}\right)$ shows a factor of 1.3. Given the simple approximation for the initial velocities, there is a reasonable agreement.

In the case where $B<0$, a monotonic potential in the sheath can be obtained with an additional condition: non-zero initial electric field. For $\alpha=\gamma=1, n_{0-}=10^{16} \mathrm{~m}^{-3}$ and $T_{-}=26 \mathrm{mV}$, the minimum absolute value of the electric field is $890 \mathrm{~V} \cdot \mathrm{m}^{-1}$. Since the potential is increasing, the electric field has to be negative. As a result of the high electric field, a double layer is seen for $x \approx 0$. The potential and the densities are shown in figure 7.10 for $x \approx 0$. It should be noted that between $x=0 \mathrm{~mm}$ and $x=0.07 \mathrm{~mm}$, the positive ion density is greater than that of the negative ions. This results in a positive space charge, immediately followed by the negative space charge of the sheath for $x>0.07 \mathrm{~mm}$. This is why we speak of a double layer. The effect of the double layer is to accelerate the negative ions until the Bohm criterion is fulfilled with equal densities. Beyond this point, the sheath is developed. Because of the double layer, these solutions do not correspond to the desired profiles. Cases with the Bohm criterion fulfilled are considered in the following sections.

\subsubsection{High Potential}

The electrode, boundary of the plasma, is polarized to a value $V_{0}$ high enough for the fraction of positive ions fast enough to overcome the potential barrier to be negligible. It was shown that this is the case for $\Phi_{L}>3$, which is equivalent to $\varphi_{L}>3 T_{-}$. A symmetrical ion-ion plasma is chosen with $m=40$ a.m.u., $n_{0}=10^{16} \mathrm{~m}^{-3}$ and $T=26 \mathrm{mV}$. The limit value of the potential is thus $\varphi_{L}=78 \mathrm{mV}$, which is very low due to the low temperature of the ions.

Figure 7.11 shows the potential and the densities as functions of position for $\varphi_{L}=$ $25 \mathrm{~V}$. The reason why the sheath starts after $x=1.5 \mathrm{~mm}$ is a numerical artifact: a very small value of the initial electric field is set to launch the sheath, but leaves the equilibrium unchanged. Here, with $E_{0}=5 \times 10^{-5} \mathrm{~V} \cdot \mathrm{m}^{-1}$, the build up for the sheath takes roughly $1.5 \mathrm{~mm}$. This potential profile was compared to the potential profile from the PIC simulations (figure 7.5) and shows a good agreement. The profile for densities is also comparable to the PIC simulations with the density of the reflected species (positive ions in this case) somewhat different: the drop in density is sharper than in the PIC simulations. As to the negative ion density (accelerated species), the end values are comparable: $2.2 \times 10^{14} \mathrm{~m}^{-3}$ for PIC and $3 \times 10^{14} \mathrm{~m}^{-3}$ for the kinetic model. The profiles for the negative ion densities are very similar.

The PIC simulations showed that the Child Law is valid in the case of an ion-ion plasma with a Bohm current. Table 7.1 compares the length of the sheath as a function of its potential drop for the PIC simulation and the kinetic model. Since the value of the central density cannot be controlled directly in the PIC simulations, the value of the central density for the kinetic model is taken from the PIC simulation in each case. The values of the sheath size are very close.

\subsubsection{Floating Potential}

An additional boundary condition is used for the determination of the floating potential

$$
\Gamma_{+}(L)=\Gamma_{-}(L) .
$$




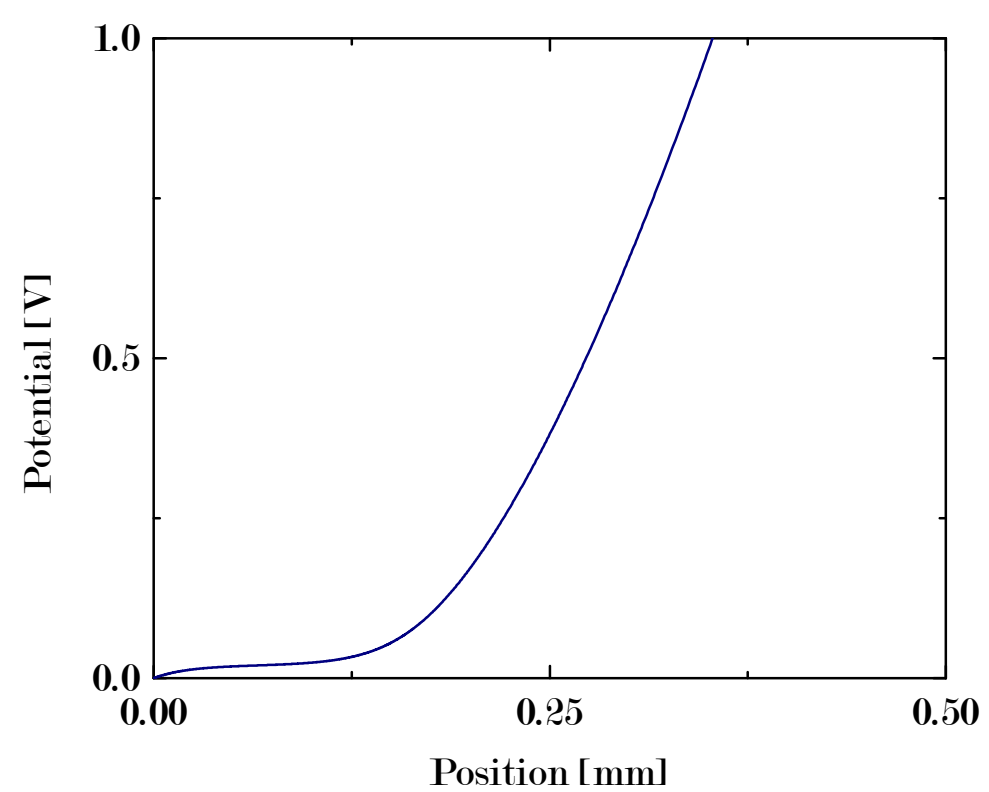

(a)

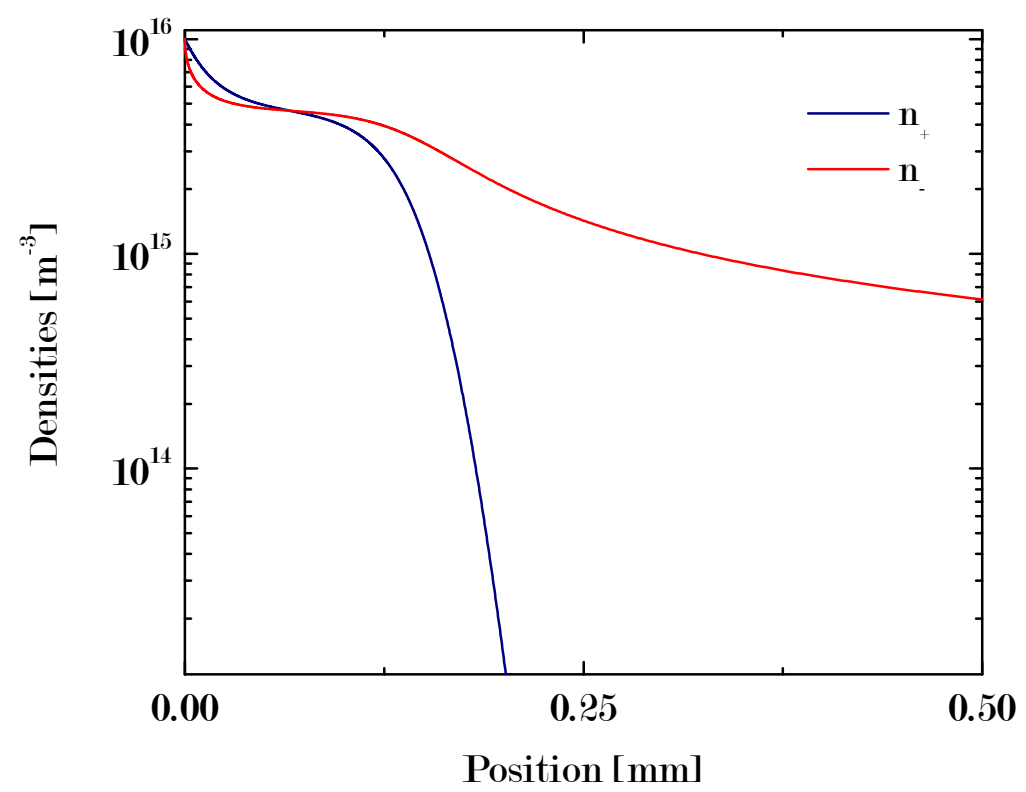

(b)

Figure 7.10: Profile of the potential (a) and the densities (b) for $x \approx 0$ with $V_{\text {inj }}=0$ and $E_{0}=-890 \mathrm{Vm}^{-1}$. The Bohm criterion is not fulfilled. Symmetrical plasma with $n_{0}=10^{16} \mathrm{~m}^{-3}, T=26 \mathrm{mV}$ and $\varphi_{L}=25 \mathrm{~V}$. 


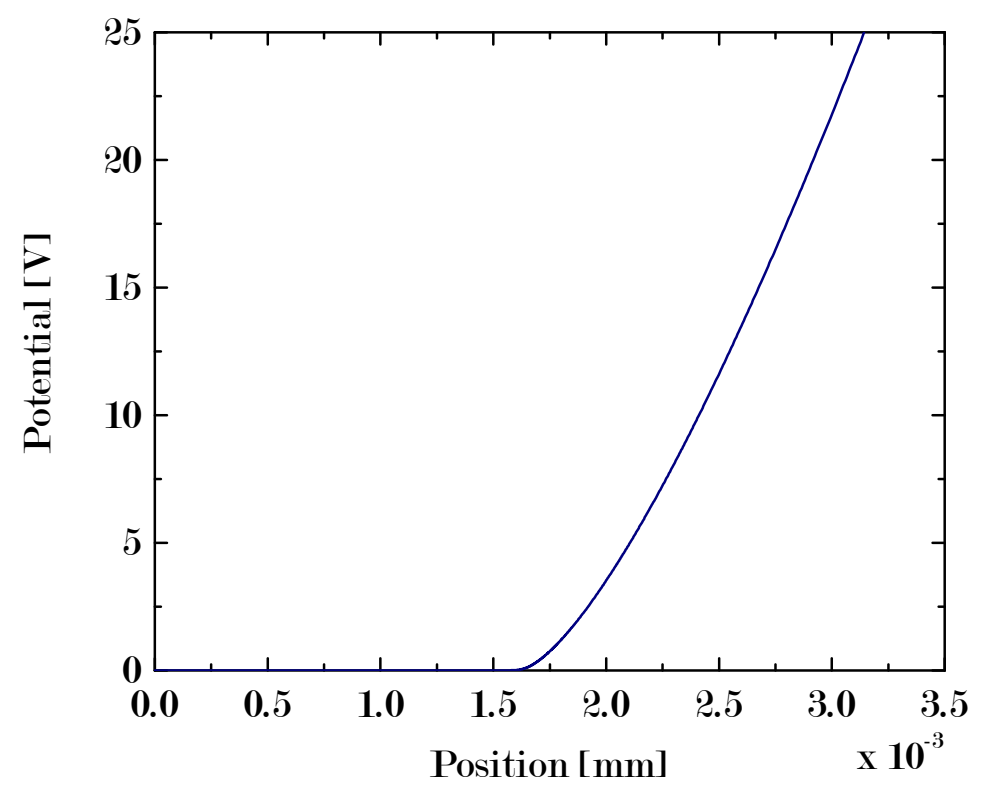

(a)

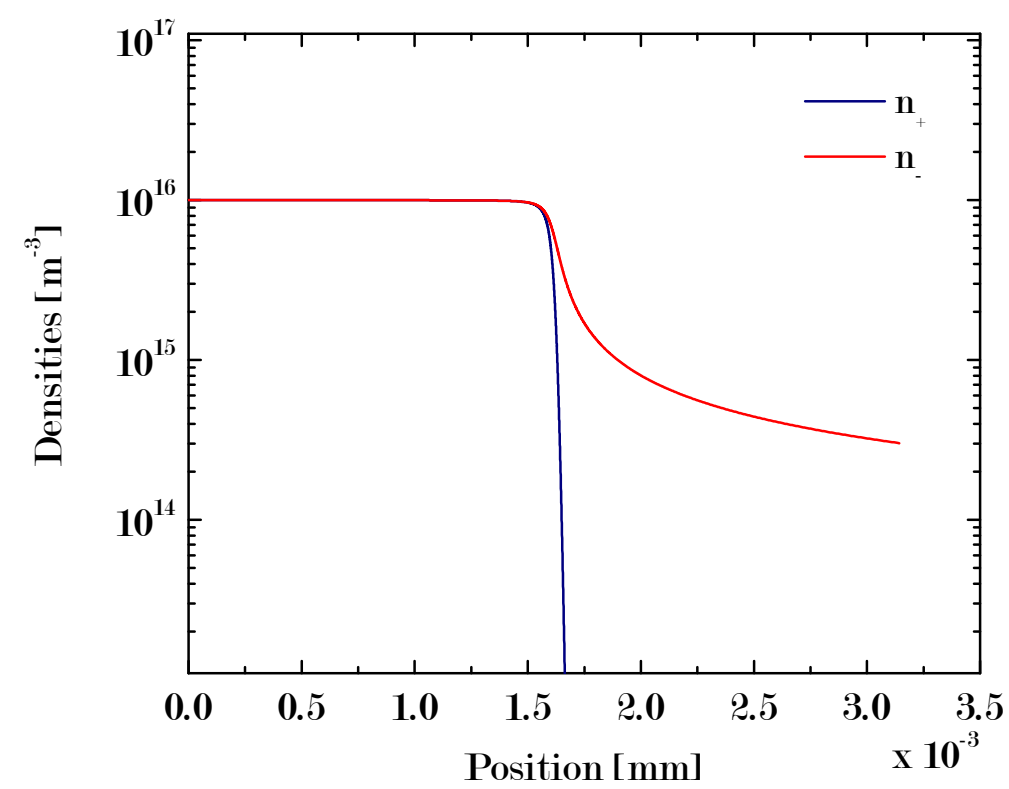

(b)

Figure 7.11: Potential (a) and densities (b) as functions of position for $m_{ \pm}=$ 40 a.m.u., $n_{0 \pm}=10^{16} \mathrm{~m}^{-3}$ and $T_{ \pm}=26 \mathrm{mV}$. The Bohm criterion is fulfilled. The end potential is set to $\varphi_{L}=25 \mathrm{~V}$. 


\begin{tabular}{c|ccccc}
\hline$V_{0}(\mathrm{~V})$ & 0.5 & 5 & 12.5 & 25 & 37.5 \\
\hline \hline PIC $s(\mathrm{~mm})$ & 0.17 & 0.68 & 1.2 & 2.1 & 3.2 \\
Kinetic $s(\mathrm{~mm})$ & 0.20 & 0.66 & 1.3 & 2.3 & 3.7 \\
\hline
\end{tabular}

Table 7.1: Sheath size as a function of its potential drop for the PIC simulations and the kinetic model. A symmetrical plasma with $m=40$ a.m.u. and $T=26 \mathrm{mV}$ is chosen; initial densities are taken from the PIC simulations.

The expression of the fluxes at $x=L$, therefore, is needed, with the definition

$$
\Gamma_{ \pm}(L)=\int_{-\infty}^{+\infty} v f_{ \pm}(L, v) d v
$$

The positive ion flux is easily obtained as

$$
\Gamma_{+}(L)=\frac{n_{0+} v_{0+}}{\sqrt{\pi}} \frac{\exp -\frac{\varphi_{L}}{T_{+}}}{1+\operatorname{erf} \sqrt{\frac{\varphi_{L}}{T_{+}}}}
$$

with $v_{0+}$ defined similarly to $v_{0-}$ in $(7.52)$ as $v_{0+}=\sqrt{\left(2 e T_{+}\right) / m_{+}}$. The negative ion flux can only be expressed as an integral

$$
\Gamma_{-}(L)=\frac{n_{0-}}{v_{0-}} \frac{2}{\sqrt{\pi}} \int_{\sqrt{v_{\mathrm{inj}}^{2}+\frac{2 e \varphi_{L}}{m_{-}}}}^{+\infty} v \exp \left[-\frac{m_{-}}{2 e T_{-}}\left(\sqrt{v-\frac{2 e \varphi_{L}}{m_{-}}}-v_{\mathrm{inj}}\right)^{2}\right] d v
$$

Using the normalization (7.52), these fluxes become

$$
\begin{aligned}
& N_{+} V_{+}\left(L / L_{0}\right)=\alpha \sqrt{\frac{\delta}{\pi \gamma}} \frac{\exp -\gamma \Phi_{L}}{1+\operatorname{erf} \sqrt{\gamma \Phi_{L}}}, \\
& N_{-} V_{-}\left(L / L_{0}\right)=\frac{2}{\sqrt{\pi}} \int_{\sqrt{V_{\mathrm{inj}}^{2}+\Phi_{L}}}^{+\infty} \exp \left[-\left(\sqrt{V-\Phi_{L}}-V_{\mathrm{inj}}\right)^{2}\right] .
\end{aligned}
$$

As mentioned previously, the floating potential of a symmetrical ion-ion plasma is zero. A parameter of asymmetry, therefore, must be chosen to study the scaling of the floating potential. The asymmetry introduced by the difference in masses is chosen, as it is interesting for electronegative plasmas. As the feedstock gas is molecular, multiple ion species with different masses can be found, e.g. $\mathrm{O}^{+}$and $\mathrm{O}_{2}^{+}$in oxygen. The ratio of masses $\delta=m_{-} / m_{+}$is chosen with $m_{-}$kept constant while $m_{+}$is varied. Following the principle of minimum energy, the injection velocity is set as the minimum injection velocity that can be calculated from (7.67) in the case of high potentials.

Numerically, the problem becomes more complex as an additional boundary condition is used: with $\varphi(L)=\varphi_{L}, \Gamma_{+}(L)=\Gamma_{-}(L)$. The value $\varphi_{L}$ is no longer an input (arbitrarily set), but an output of the problem. A shooting method with $\varphi_{L}$ as parameter is chosen to solve the equation (7.56). Trying to abide by the full Bohm criterion from (7.66) with a dependence on $\varphi_{L}$ would make the problem too difficult to solve.

The study is done with $n_{0 \pm}=10^{16} \mathrm{~m}^{-3}, T_{ \pm}=26 \mathrm{mV}$ and $m_{-}=40$ a.m.u. With a fixed value of $m_{-}$, the value of the injection velocity is also fixed: $v_{\text {inj }}=131 \mathrm{~m} \cdot \mathrm{s}^{-1}$. Figure 7.12 shows the scaling of the floating potential normalized to the temperature as 


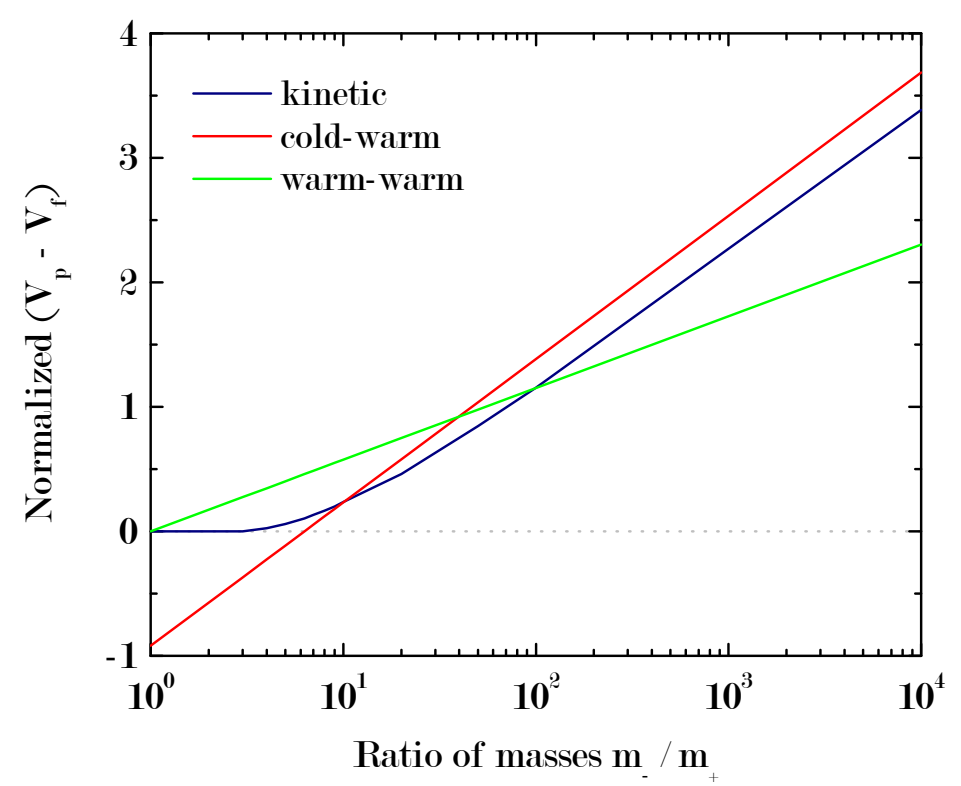

Figure 7.12: $\left(V_{p}-V_{f}\right)$ normalized to the temperature as a function of mass ratio $m_{-} / m_{+}$for the kinetic model (blue), the cold-warm case (red) and the warm-warm case (green). The $V=0$ curve is also shown as a dotted line.

a function of mass ratio $m_{-} / m_{+}$for three cases: the kinetic simulations, the cold-warm case, and the warm-warm case. As expected from its expression (7.27), the curve for the cold-warm case is negative for values of the ratio $\delta<2 \pi$. The warm-warm case shows the correct value $V_{p}-V_{f}=0$ for $\delta=1$. As to the kinetic model, it shows the transition from the $\delta=1$ case with $V_{p}-V_{f}=0$ to the case of high mass ratios correctly described by the cold-warm approximation. For high mass ratios, the agreement between the kinetic model and the cold-warm approximation is fairly good, although the kinetic theory predicts smaller values of $V_{p}-V_{f}$. For small mass ratios, a discrepancy is seen between the kinetic model and the warm-warm approximation. It is difficult to determine which model describes more accurately the floating potential. The difference could be due to the simple approximation of the initial distribution functions in the kinetic model shown in figure 7.9. However, it could be argued that the warm-warm description is not fitting to the context where one of the species is accelerated. The assumption of a Boltzmann equilibrium is usually reserved to species confined to the plasma.

\subsection{Conclusions}

The sheath structure for ion-ion plasmas was investigated with simple fluid models, PIC simulation, and a kinetic model.

The first important feature shown is the fact that a Bohm criterion needs to be fulfilled at the sheath edge: the species to be accelerated in the sheath needs to be pre-accelerated to the ion Bohm velocity $u_{B \pm}=\sqrt{e T_{\mp} / m_{ \pm}}$. This is similar to the sheath formation in electron-positive ion plasmas. PIC simulations showed the Bohm criterion with the average velocity of the accelerated species at the sheath matching the expression of the ion Bohm velocity. In the context of the kinetic model, it was shown that a minimal injection velocity $v_{\text {inj }}$ shifting the distribution function was needed as the Bohm criterion 
cannot be fulfilled with $v_{\text {inj }}=0$.

The case of high voltage sheaths corresponds to the extraction of ions with a grid biased to a high voltage in order to reach high velocities $\left(\mathrm{I}_{\mathrm{sp}}\right)$. Here, as one of the species is reflected, the symmetry of the ion-ion plasma does not play a role and the situation is best described with the cold-warm fluid approximation. This approximation is usually applied to electron-positive ion plasmas.

The case of low voltage sheaths corresponds to the small potential difference in front of floating conductors or dielectric surfaces, and was studied as a function of mass ratio. As the thruster cylinder is a dielectric, the potential structure is described by the floating potential. The cold-warm approximation can only be used for high mass ratios where the ion-ion plasma is no longer symmetrical. The warm-warm approximation describes the situation at low mass ratios: the ion-ion plasma can be considered symmetrical as the difference between the ion masses is small. The kinetic model showed the transition between the two regimes. 


\section{Chapter 8}

\section{Conclusions and Future Work}

\section{Contents}

8.1 Ionization Stage $\ldots \ldots \ldots \ldots$. . . . . . . . . . . . . .

8.2 Magnetic Electron Filtering Stage . . . . . . . . . 166

8.3 Extraction Stage ................. 167

8.4 Future Work . . . . . . . . . . . . . . 167

8.4.1 What Could Not Be Done . . . . . . . . . . . . . . 167

$8.4 .2 \quad$ PEGASES Prototype II . . . . . . . . . . . . . . 168

The PEGASES thruster presented in this thesis is a new plasma thruster concept. Its name stands for Plasma Propulsion with Electronegative GASES. In a classical thruster, the thrust is produced by accelerating positive ions from an electron-positive ion plasma. In the PEGASES concept, an electronegative plasma is used, with electrons, positive and negative ions. Both positive and negative ions are used to create the thrust. Several advantages follow from this design, among which the fact that an electron emitting hollow cathode is no longer needed to neutralize the downstream ion beam. This is a consequence of the total exiting ion flux being neutral to begin with (positive and negative ions).

The PEGASES thruster can be divided into three stages: the ionization stage, the magnetic electron filtering stage, and the extraction and acceleration stage. In the first stage, the electronegative plasma is excited by helicon waves, launched with a radiofrequency excited antenna. In the second stage, electrons are spatially filtered with a static magnetic field to obtain an ion-ion plasma (electron free). In the third stage, both ion species are extracted and accelerated. This is made possible by the obtainment of an ion-ion plasma in the previous stage. The first two stages were investigated experimentally in the first PEGASES thruster prototype using electrostatic probes and retarding-field energy analyzers. The last two stages were investigated theoretically with fluid and kinetic models. With the results from all stages, a better understanding of the PEGASES concept stages was achieved. This allowed the design of the second PEGASES thruster prototype.

\subsection{Ionization Stage}

After several modifications to the original PEGASES thruster design, high densities were reached with the helicon mode.

Several aspects of the ionization stage were found: 
- The design of a double helicon for the PEGASES thruster is inappropriate as asymmetries and instabilities were observed and could not be controlled. A simpler design with only one extraction zone should be used to avoid these issues.

- An optimum of the positive ion flux as a function of magnetic field strength and gas flow was measured. The influence of the plasma conditions (gas, applied power, magnetic field and gas flow) was investigated and understood.

- A good mass efficiency in argon was measured, with a maximum over $40 \%$ at high power $(P=1.4 \mathrm{~kW})$.

- The drawback of using molecular gases $\left(\mathrm{O}_{2}, \mathrm{SF}_{6}, \mathrm{I}_{2}\right)$, necessary for the PEGASES thruster, was found and understood. As more energy is lost per electron-ion pair creation, the resulting plasma density is lower than that of atomic gases (Ar).

\subsection{Magnetic Electron Filtering Stage}

In the experiments, key aspects of the magnetic electron filtering came to be understood:

- The first role of the magnetic field is to confine electrons (core in a cylindrical geometry). It allows the obtention of an electron-free region.

- The second role of the magnetic field is to create a strong electron temperature gradient. Since electrons diffuse cross-field through collisions, they lose energy and their temperature decreases. Thus, there are two regions. In the high electron temperature region, the creation of positive ions is efficient. In the low electron temperature region, the electron attachment yielding negative ions is efficient.

- Neutral molecules are needed in the low electron temperature region since the attachment is dissociative. In the first PEGASES thruster prototype, mainly atoms were found in this region due to the injection of neutrals in the high electron temperature region. A patent on neutral injection optimization was filed [27].

The fluid model showed that the electron and positive ion losses in the direction of magnetic field lines are an important aspect of the magnetic filtering. Not only are electrons confined, but they are lost at the end walls. The scaling of the optimized negative ion flux was obtained for low electronegativity

$$
\left.\Gamma_{-}(R)\right|_{\mathrm{opt}} \propto \frac{K_{\mathrm{att}}}{K_{\mathrm{rec}}} \frac{B_{0}^{2}}{n_{g}} \frac{R^{2}}{L},
$$

and high electronegativity

$$
\left.\Gamma_{-}(R)\right|_{\text {opt }} \propto \frac{K_{\text {att }}}{K_{\text {rec }}} \frac{B_{0}^{4}}{n_{g}^{3}} \frac{R^{3}}{L^{2}} .
$$

In both cases, the negative ion flux can be increased through a higher attachment rate (stronger electronegativity), or through a higher magnetic field (enhanced electron confinement). An increase in pressure (neutral density) reduces the negative ion flux, with a stronger effect at high electronegativity. As expected, the negative ion flux is a decreasing 
function of the recombination rate. The scaling of the thruster radius in the considered geometry was also calculated

$$
R \propto \frac{(R L)^{1 / 2} n_{g}}{B_{0} \bar{\alpha}^{1 / 2}}
$$

where the influence of the geometry $(R L)$, the pressure $\left(n_{g}\right)$, the magnetic field $\left(B_{0}\right)$, and the electronegativity $(\alpha)$ can be seen.

\subsection{Extraction Stage}

The sheaths structure in ion-ion plasmas was investigated:

- A Bohm criterion needs to be fulfilled and ensures that the potential in the sheath is monotonic. The Bohm velocity in ion-ion plasmas $u_{B \pm}=\sqrt{e T_{\mp} / m_{ \pm}}$is similar to the Bohm velocity in electron-positive ion plasmas $u_{B}=\sqrt{e T_{e} / m_{+}}$.

- The behavior of the sheaths for high voltages was found to be similar to the electronpositive ion sheaths. The Child law, with scalings between the sheath size, potential difference, and current, describes high voltage sheaths whether the plasma is composed of electrons and positive ions, or positive and negative ions.

- The floating potential was investigated as a function of mass ratio between the ion species. For high mass ratios, the cold-warm fluid approximation (electron-positive ion case) gives the correct scaling for the potential difference $V_{p}-V_{f}$. For small mass ratios, the warm-warm fluid approximation shows the correct value $V_{p}-V_{f}=0$ when the ion masses are equal. The kinetic model showed the transition between the two regimes.

\subsection{Future Work}

\subsubsection{What Could Not Be Done}

Ionization Stage The study of the ion fluxes with a retarding field energy analyzer would be interesting to compare to the measurements done in the helicon reactor, especially in the understanding of the plasma potential in an ion-ion plasma. As electrons travel fast along the magnetic field lines, the plasma potential depends on the potential on the cylinder ends. Biasing the metal plates that close the cylinder should have interesting effect on the plasma diffusion.

Magnetic Filtering Stage In the experiments, the gradient of electron temperature was found to play a key role in the ion-ion plasma obtention. A profile of electron temperature should thus be used with the same equations. In order to obtain higher ion densities and fluxes, a stronger attachment rate should also be considered as oxygen is weakly electronegative.

Extraction and Acceleration Stage An accelerating grid was designed and will be tested. It consists of a single hole to understand the behavior of the accelerated ions. Only one extraction grid is used, with an alternative bias to accelerate the ion species one 
after the other. The multi-planar probe will be used to estimate the ion flux profile over the area of the extraction zone.

\subsubsection{PEGASES Prototype II}

The design of the second PEGASES thruster prototype is based on what was learned from the first prototype.

Due to the limitations of the cylindrical geometry, the choice of a linear geometry is made: all stages of the thruster, from the gas injection to the acceleration of ions are along the same axis. The magnetic filter separates the thruster into two regions. It was found that the magnetic field should be stronger to properly confine the electrons. Also, to avoid symmetry issues, only one extraction zone is considered (along the axis). An optimized neutral injection profile will be used, guaranteeing that neutral molecules are present in the low electron temperature region. 


\section{Appendix A}

\section{Relation Between Edge and Average Electronegativity}

A relation between $\alpha_{s}$ and $\bar{\alpha}$ is needed to determine all variables. First, let us consider all balance equations with a drift-diffusion approximation, keeping in mind that quasineutrality $n_{+}=n_{e}+n_{-}$is assumed:

$$
\begin{aligned}
& \Gamma_{e}=-D_{\perp e} \frac{d n_{e}}{d x}-\mu_{\perp e} n_{e} E \\
& \Gamma_{+}=-D_{+} \frac{d n_{e}}{d x}-D_{+} \frac{d n_{-}}{d x}+\mu_{+}\left(n_{e}+n_{-}\right) E, \\
& \Gamma_{-}=-D_{+} \frac{d n_{-}}{d x}-\mu_{+} n_{-} E .
\end{aligned}
$$

Here, the drift term is no longer assumed negligible compared to the diffusion term. The ion drift and diffusion coefficients are taken to be the same because of the assumption of the model: $m_{+}=m_{-}, T_{+}=T_{-}$and $\nu_{+}=\nu_{-}$. Since a one-dimensional fluid model is considered, $\Gamma_{+}=\Gamma_{e}+\Gamma_{-}$, which can be combined with all three approximated driftdiffusion equations

$$
-D_{+} \frac{d n_{e}}{d x}-D_{+} \frac{d n_{-}}{d x}+\mu_{+}\left(n_{e}+n_{-}\right) E=-D_{\perp e} \frac{d n_{e}}{d x}-\mu_{\perp e} n_{e} E-D_{+} \frac{d n_{-}}{d x}-\mu_{+} n_{-} E,
$$

which leads to the expression of the electric field

$$
E=\frac{D_{+}-D_{\perp e}}{2 \mu_{+} n_{-}+\left(\mu_{+}+\mu_{\perp e}\right) n_{e}} \frac{d n_{e}}{d x} .
$$

Inserting this expression into the negative ion drift-diffusion equation (A.3) gives

$$
\Gamma_{-}=-D_{+} \frac{d n_{-}}{d x}-\frac{\mu_{+} n_{-}\left(D_{+}-D_{\perp e}\right)}{2 \mu_{+} n_{-}+\left(\mu_{+}+\mu_{\perp e}\right) n_{e}} \frac{d n_{e}}{d x} .
$$

$\Gamma_{-}(x)$ and $n_{e}(x)$ were previously calculated in (6.77) and (6.71), while the negative ion density is assumed constant with $n_{-}=\bar{n}_{-}$:

$$
\begin{aligned}
-\frac{2 D_{+}}{n_{e 0}} \frac{d n_{-}}{d x}= & -\frac{D_{+}-D_{\perp e}}{2+\left(1+\mu_{\perp e} / \mu_{+}\right)[1+\cos (\pi x / R)](2 \bar{\alpha})^{-1}} \frac{\pi}{R} \sin \frac{\pi x}{R} \\
& +\left[\nu_{\mathrm{att}}-K_{\mathrm{rec}} n_{e 0} \bar{\alpha}(1+2 \bar{\alpha})\right] x \\
& +\left(\nu_{\mathrm{att}}-K_{\mathrm{rec}} n_{e 0} \bar{\alpha}\right) \frac{R}{\pi} \sin \frac{\pi x}{R} .
\end{aligned}
$$


This equation can be integrated between $x=0$ and $x=R$ since all variables are known with

$$
\int \frac{A \sin \omega x}{B+C(1+\cos \omega x)} d x=-\frac{A}{C \omega} \ln (B+C(1+\cos \omega x)),
$$

with the rescaling $u=\cos \omega x, d u=-\sin \omega x$. The result of this integration is

$$
\begin{aligned}
-D_{+}\left(\alpha_{s}-\alpha_{0}\right)=-\frac{D_{+}-D_{\perp e}}{1+\mu_{\perp e} / \mu_{+}} \bar{\alpha} \ln \left[1+\frac{1+\mu_{\perp e} / \mu_{+}}{2 \bar{\alpha}}\right] \\
+\left[\nu_{\mathrm{att}}-K_{\mathrm{rec}} n_{e 0} \bar{\alpha}(1+2 \bar{\alpha})\right] \frac{R^{2}}{4}+\left[\nu_{\mathrm{att}}-K_{\mathrm{rec}} n_{e 0} \bar{\alpha}\right] \frac{R^{2}}{\pi^{2}}
\end{aligned}
$$

Equation (A.9), for the set of parameters considered, is a relation between $\alpha_{0}, \alpha_{s}$ and $\bar{\alpha}$. Since $\bar{\alpha}$ is the only input among those $\alpha$ 's, an additional relation is needed. Taking a power series expansion of the right hand side terms of equation (A.7), then integrating gives

$$
\frac{d n_{-}}{d x}(x)=A x+o(x), \quad n_{-}(x)=n_{n 0}+\frac{A}{2} x^{2}+o\left(x^{2}\right) .
$$

Approximating $\alpha(x)=n_{-}(x) / n_{e 0}$ to the second order and using the boundary conditions $\alpha(0)=\alpha_{0}$ and $\alpha(R)=\alpha_{s}$ yields

$$
\alpha(x)=\frac{\alpha_{s}-\alpha_{0}}{R^{2}} x^{2}+\alpha_{0}
$$

By definition, $\bar{\alpha}=\left(\int_{0}^{R} \alpha(x) d x\right) / R$, which gives the second relation between the $\alpha$ 's,

$$
\bar{\alpha}=\frac{2}{3} \alpha_{0}+\frac{1}{3} \alpha_{s}
$$

Eliminating $\alpha_{0}$ in (A.9) using (A.12), $\alpha_{s}$ is obtained in terms of $\bar{\alpha}$

$$
\begin{aligned}
&-\frac{3 D_{+}}{2} \alpha_{s}=-\frac{3 D_{+}}{2} \bar{\alpha}- \frac{D_{+}-D_{\perp e}}{1+\mu_{\perp e} / \mu_{+}} \bar{\alpha} \ln \left[1+\frac{1+\mu_{\perp e} / \mu_{+}}{2 \bar{\alpha}}\right] \\
&+\left[\nu_{\mathrm{att}}-K_{\mathrm{rec}} n_{e 0} \bar{\alpha}(1+2 \bar{\alpha})\right] \frac{R^{2}}{4}+\left[\nu_{\mathrm{att}}-K_{\mathrm{rec}} n_{e 0} \bar{\alpha}\right] \frac{R^{2}}{\pi^{2}}
\end{aligned}
$$




\section{Bibliography}

[1] deGrasse Tyson N, On Being Rarefied, Natural History Magazine, February 1998

[2] Spitzer L Jr, History of the Space Telescope, Q. Jl R. astr. Soc., 20, 29-36, 1979

[3] Newton I, The Principia, translated by Cohen I B and Whitman A, University of California Press, Berkeley, 1999

[4] Goebel D M and Katz I, Fundamentals of Electric Propulsion: Ion and Hall Thrusters, JPL Space Science and Technology Series, Wiley, 2008

[5] Sutton G P and Biblarz O, Rocket Propulsion Elements, 7th Edition, WileyInterscience, 2000

[6] Ariane Espace, Ariane 5 - User's Manual, Issue 5, Revision 0, July 2008

[7] Lieberman M A and Lichtenberg A J, Principles of Plasma Discharges and Materials Processing, 2nd edition, New York: Wiley-Interscience, 2005

[8] Lieberman M A, Booth J-P, Chabert P, Rax J-M and Turner M M, Standing Waves and Skin Effects in High Frequency Capacitive Discharges, Plasma Sources Sci. Technol., 11, 283-293, 2002

[9] Perret A, Chabert P, Booth J-P, Jolly J, Guillon J and Auvray P, Ion Flux NonUniformities in Large Area High Frequency Capacitive Discharges, Appl. Phys. Lett., 83, 243-245, 2003

[10] Chabert P, Raimbault J-L, Levif $\mathrm{P}$ and Rax J-M, Inductive Heating and E to $H$ Transitions in Capacitive Discharges, Phys. Rev. Lett., 95, 205001, 2005

[11] Boswell R W, Plasma Production Using a Standing Helicon Wave, Phys. Lett. A, 33, 457-458, 1970

[12] Boswell R W, Very Efficient Plasma Generation by Whistler Waves Near the Lower Hybrid Frequency, Plasma Phys. Controlled Fusion, 26, 1147-1162, 1984

[13] Boswell R W and Chen F F, Helicons - The Early Years, IEEE Trans. Plasma Science, 25, 1229-1244, 1997

[14] Chen F F and Boswell R W, Helicons - The Past Decade, IEEE Trans. Plasma Science, 25, 1245-1257, 1997 
[15] Welander B, Carpenter C, de Grys K, Hofer R R, Randolph T M and Manzella T $\mathrm{H}$, Life and Operating Range Extension of the BPT-4000 Qualification Model Hall Thruster, AIAA 2006-5263, 42 ${ }^{\text {nd }}$ AIAA/ASME/SAE/ASEE Joint Propulsion Conference \& Exhibit, Sacramento, California, United States, July 9-12, 2006

[16] Jahn R G and Choueri E Y, Electric Propulsion, Encyclopedia of Physical Science and Technology, Third Edition, Volume 5, 125-141, 2002

[17] Chang Diaz F R, An Overview of the VASIMIR Engine: High Power Space Propulsion with RF Plasma Generation and Heating, AIP Conf. Proc., 595, 3-15, 2001

[18] Squire J P, Chang Diaz F R, Carter M D, Cassady L D, Chancery W J, Glover T W, Jacobson V T, McCaskill G E, Bengtson R D, Bering E A and Deline C A, High Power VASIMIR Experiments Using Deuterium, Neon and Argon, IEPC-2007-181, $30^{\text {th }}$ International Electric Propulsion Conference, Florence, Italy, September 17-20, 2007

[19] Charles C and Boswell R W, A Plasma Beam Generator, patent \#PS3033/02, filed on June 19, 2002

[20] Block L P, A Double Layer Review, Astrophysics and Space Science, 55, 59-83, 1978

[21] Plihon N, Stabilité et Structure Electrique d'une Décharge Inductive en Gaz Electronégatif, PhD Thesis, Ecole Polytechnique, 2006

[22] Plihon N, Chabert P and Raimbault J-L, Helicon Double Layer Thruster Concept for High Power NEP Missions, Tech, Rept. ESA - Ariadna Study ACT-04-3101, 2005

[23] Chabert P, Propulseur a Plasma Electronegatif, patent \#05 12417, filed on December 7, 2005

[24] Emeleus K G and Woolsey G A, Discharges in Electronegative Gases, Taylor \& Francis, 1970

[25] Nigham W L and Wiegand W J, Influence of Negative-Ion Processes on Steady State Properties and Striations in Molecular Gas Discharges, Phys. Rev. A, 10, 922-945, 1974

[26] Johnson J A and Ramaiah R, Plasma Instability in the Presence of Negative Ions, Phys. Rev. A, 36, 774-794, 1987

[27] Chabert P, Aanesland A, Meige A and Leray G, patent \#08 58077, filed on November 28,2008

[28] Dawson J M, Particle Simulation of Plasmas, Rev. Mod. Phys., 55, 403-447, 1983

[29] Mott-Smith H M and Langmuir I, The Theory of Collectors in Gaseous Discharges, Phys. Rev., 28, 727-763, 1926

[30] Chen F F, Lecture Notes on Langmuir Probe Diagnostics, IEEE-ICOPS Meeting, Jeju, Korea, June 5, 2003 
[31] Rubinstein J and Laframboise J G, Theory of Axially Symmetric Probes in a Collisionless Magnetoplasma: Aligned Spheroids, finite cylinders, and Disks, Phys, Fluids, 26, 3624-3629, 1983

[32] Demidov V I, Ratynskaia S V, Armstrong R J and Rypdal K, Probe Measurements of Electron Energy Distributions in a Strongly Magnetized Low-Pressure Helium Plasma, Phys. Plasmas, 6, 350-358, 1999

[33] Dine S, Effet de la Fréquence dans les Décharges VHF sur les Caractéristiques des Plasmas Utilisés pour le Dépôt de Silicium Cristallin, PhD Thesis, Université Paris XI, 2006

[34] Ingram S G and Braithwaite N S J, Ion and Electron Energy Analysis at a Surface in a RF Discharge, J. Phys. D: Appl. Phys., 21, 1496-1503, 1988

[35] Bohm C and Perrin J, Retarding Field Analyzer for Measurements of Ion Energy Distributions and Secondary Electron Emission Coefficients in Low Pressure, Rev. Sci. Instr., 64, 31-44, 1993

[36] Orfanidis S J, Introduction to Signal Processing, Prentice-Hall, 1996

[37] Bacal M, Hamilton G W, Bruneteau A M, Doucet J and Taillet J, Measurement of $H^{-}$Density in Plasma by Photodetachment, Rev. Sci. Instrum., 50, 719-721, 1979

[38] Bacal M, Photodetachment Diagnostic Techniques for Measuring Negative Ion Densities and Temperatures in Plasmas, Rev. Sci. Instrum., 71, 3981-4007, 2000

[39] Sheridan T E, Chabert P and Boswell R W, Positive Ion Flux from a Low-Pressure Electronegative Discharge, Plasma Source Sci. Technol., 8, 457-462, 1999

[40] Overzet L J, Smith B A, Kleber J and Kanakasabapathy S K, Negative Ion Extraction from Pulsed Discharges, Jpn. J. Appl. Phys., 36, 2443-2449, 1997

[41] D'Angelo N, Goeler S v and Ohe T, Propagation and Damping of Ion Waves in a Plasma with Negative Ions, Phys. Fluids, 3, 1605-1606, 1966

[42] St-Onge L, Margot J and Chaker M, Characterization of the Negative Ion Fraction in High-Density SF 6 Magnetoplasmas Using Ion Acoustic Waves, Appl. Phys. Lett., 72, 290-292, 1998

[43] Chabert $\mathrm{P}$, Etude d'un Plasma de $S F_{6}$ créé dans un réacteur hélicon: application à la gravure du carbure de silicium, PhD Thesis, Université Paris XI, 1999

[44] Chabert P, Sheridan T E, Boswell R W and Perrin J, Electrostatic Probe Measurement of the Negative Ion Fraction in an $\mathrm{SF}_{6}$ Helicon Discharge, Plasma Source Sci, Technol., 8, 561-566, 1999

[45] Corr C S, Plihon N and Chabert P, Transition from Unstable Electrostatic Confinement to Stable Magnetic Confinement in a Helicon Reactor Operating with Ar/SF 6 Gas Mixtures, J. Appl. Phys/, 99, 103302, 2006

[46] Finite Element Method Magnetics, http://www.femm.info 
[47] Stix T H, The Theory of Plasma Waves, New York: McGraw-Hill, 1962

[48] Hartree D R, The Propagation of Electromagnetic Waves in a Refracting Medium in a Magnetic Field, Math. Proc. Cambridge Phil. Soc., 27, 143-162, 1931

[49] Alfvén H, Existence of Electromagnetic-Hydrodynamic Waves, Nature, 150, 405-406, 1942

[50] Aigrain P, Proc. Int. Conf. Semiconductor Physics, 224, Prague, Czechoslovakia, 1960

[51] Chen FF, High Density Plasma Souces, chapter 1: Helicon Plasma Sources, William Andrew, 1997

[52] Watari T, Hatori T, Kumazawa R, Hidekuma S, Aoki T, Kawamoto T, Inutake M, Hiroe S, Nishizawa A, Adati K, Sato T, Watanabe T, Obayashi H and Takayama K, Radio-Frequency Plugging of a High Density Plasma, Phys. Fluids, 21, 2076-2081, 1978

[53] Shoji T, IPPJ Annu. Rep., Nagoya Univ., page 67, 1986

[54] Fruchtman A, Makrinich G, Chabert P and Rax J-M, Enhanced Plasma Transport Due to Neutral Depletion, Phys. Rev. Lett., 96, 065002, 2005

[55] Raimbault J-L, Liard L, Rax J-M, Chabert P, Fruchtman A and Makrinich G, Steady-State Isothermal Bounded Plasma with Neutral Dynamics, Phys. Plasmas, 14, 013503, 2007

[56] Liard L, Raimbault J-L, Rax J-M and Chabert P, Plasma Transport under Neutral Gas Depletion Conditions, J. Phys. D: Appl. Phys., 40, 5192-5195, 2007

[57] Aanesland A, Liard L, Leray G, Jolly J and Chabert P, Direct Measurements of Neutral Density Depletion by Two-Photon Absorption Laser-Induced Fluorescence Spectroscopy, Appl. Phys. Lett., 91, 121502, 2007

[58] Liard L, Raimbault J-L and Chabert P, Competitive Effects of an Axial Magnetic Field and of Neutral Gas Depletion in a Positive Column, Phys. Plasmas, 16, 053507, 2009

[59] Smith D, Dean A G and Adams N G, Space Charge Fields in Afterglow Plasmas, J. Phys. D: Appl. Phys., 7, 1944-1962, 1974

[60] Kaganovich I D, Economou D J, Ramamurthi B N and Midha V, Negative Ion Density Fronts during Ignition and Extinction of Plasmas in Electronegative Gases, Phys. Rev. Lett., 84, 1918-1921, 2000

[61] Kaganovich I D, Ramamurthi B N and Economou D J, Spatiotemporal Dynamics of Charged Species in the Afterglow of Plasmas Containing Negative Ions, Phys. Rev. E, 64, 036402, 2001

[62] Malyshev M V, Donnelly V M, Colonell J I and Samukawa S, Dynamics of PulsedPower Chlorine Plasmas, J. Appl. Phys, 86, 4813-4820, 1999 
[63] Ahn T H, Nakamura K and Sugai H, Negative Ion Measurements and Etching in a Pulsed-Power Inductively Coupled Plasma in Chlorine, Plasma Sources Sci. Technol., 5, 139-144, 1996

[64] Hebner G A and Fleddermann C B, Characterization of Pulse-Modulated Inductively Coupled Plasmas in Argon and Chlorine, J. Appl Phys., 82, 2814-2821, 1997

[65] Walton S G, Leonhardt D, Fernsler R F and Meger R A, Extraction of Positive and Negative Ions from Electron-Beam-Generated Plasmas, Appl. Phys. Lett., 81, 987-989, 2002

[66] Stamate E and Ohe K, Determination of Negative-Ion and Electron Parameters in an $\mathrm{Ar} / S F_{6}$ Plasma, J. Appl. Phys., 84, 2450-2458, 1998

[67] Kawai R and Mieno T, Effective Production of Negative Ions around Magnetized $\mathrm{CF}_{4}$ Plasma Column, Jpn. J. Appl. Phys., 36, L 1123-1125, 1997

[68] Chabert P, Aanesland A, Meige A and Leray G, patent \#08 58077, filed on November 28,2008

[69] Franklin R N and Snell J, The Positive Column with Negative Ions in a Magnetic Field at Low Pressure, J. Phys. D: Appl. Phys., 32, 1031-1038, 1999

[70] Forrest J R and Franklin R N, The Positive Column in a Magnetic Field at Low Pressures: the Transition from Free-Fall to Ambipolar Conditions, Br. J. Appl. Phys., 17, 1569-1574, 1966

[71] Forrest J R and Franklin R N, The Theory of the Positive Column Including SpaceCharge Effects, J. Phys. D: Appl. Phys, 1, 1357-1368, 1968

[72] Leray G, Chabert P, Lichtenberg A J and Lieberman M A, Fluid Model of an Electronegative Discharge with Magnetized Electrons and Unmagnetized Ions, J. Phys. D: Appl. Phys., 42, 194020, 2009

[73] Kimura T, Lichtenberg A J and Lieberman M A, Modelling Finite Cylinder Electronegative Discharges, Plasma Sources Sci. Technol., 10, 430-439, 2001

[74] Lichtenberg A J, Kouznetsov I G, Lee Y T, Lieberman M A, Kaganovich I D and Tsendin L D, Modelling Plasma Discharges at High Electronegativity, Plasma Sources Sci. Technol., 6, 437-449, 1997

[75] Riemann K-U, The Bohm Criterion and Sheath Formation, J. Phys. D: Appl. Phys., 24, 493-518, 1991

[76] Meige A, Leray G, Raimbault J-L and Chabert P, Sheath and Pre-Sheath in Ion-Ion Plasmas via Particle-In-Cell Simulation, Appl. Phys. Lett., 92, 061501, 2008

[77] Hockney R W and Eastwood J W, Computer Simulations Using Particles, IOP, Bristol, 1988

[78] Birdsall C K and Langdon A B, Plasma Physics via Computer Simulation, IOP, Bristol, 1991 
[79] Shampine L F, Vectorized Adaptive Quadrature in Matlab, J. Comput. and Appl. Math., 211, 131-140, 2008

[80] Chen F F, Plasma Physics and Controlled Fusion, Volume 1. Plasma Physics, 2nd edition, Springer, 2006 


\title{
Sheath and presheath in ion-ion plasmas via particle-in-cell simulation
}

\author{
A. Meige, ${ }^{\text {a) }}$ G. Leray, J.-L. Raimbault, and P. Chabert \\ Laboratoire de Physique et Technologie des Plasmas (LPTP), Ecole Polytechnique, Palaiseau Cedex 91128, \\ France
}

(Received 3 July 2007; accepted 5 January 2008; published online 11 February 2008)

\begin{abstract}
A full particle-in-cell simulation is developed to investigate electron-free plasmas constituted of positive and negative ions under the influence of a dc bias voltage. It is shown that high-voltage sheaths following the classical Child-law sheaths form within a few microseconds (which corresponds to the ion transit time) after the dc voltage is applied. It is also shown that there exists the equivalent of a Bohm criterion where a presheath accelerates the ions collected at one of the electrodes up to the sound speed before they enter the sheath. From an applied perspective, this leads to smaller sheaths than one would expect. (C) 2008 American Institute of Physics.
\end{abstract}

[DOI: $10.1063 / 1.2838293]$

Ion-ion plasmas are plasmas that consist of positive and negative ions only (electron-free plasmas); in practice, a small amount of electrons can exist, provided that the main negative charge carriers remain the negative ions. These plasmas have been investigated both experimentally ${ }^{1-4}$ and theoretically. ${ }^{5-8}$ Ion-ion plasmas may be formed in the afterglow of pulsed discharges in electronegative plasmas, ${ }^{9,10}$ in electron-beam-generated plasmas, ${ }^{11,12}$ by magnetic filtering of electronegative plasmas, ${ }^{2,4,13}$ etc. Ion-ion plasmas have many potential applications. In particular, Kanakasabapathy et $a{ }^{14}{ }^{14}$ and Walton et al. ${ }^{11,12}$ have shown that comparable fluxes of positive and negative ions can be extracted from an ion-ion plasma using a low-frequency sinusoidal bias, which could be useful in material processing to minimize charging in the fabrication of microelectronic devices. Ion-ion plasmas are also important in negative ion sources, dusty plasmas, and in the D layer of the atmosphere (see Ref. 15 and references therein). Finally, it was also recently proposed by Chabert $^{16}$ that ion-ion plasmas are a promising solution for electric space propulsion. Midha and Economou ${ }^{6}$ and Midha et $a l^{7}$ have developed a time-dependent fluid model to investigate the dynamics of an ion-ion plasma under the influence of a rf and a dc voltage and found that the sheath structure differed profoundly from conventional electron-ion plasmas. However, the size of the sheath and existence of a presheath were not investigated in details. The sheath, the presheath, and their formation are fundamental features in the design of extracting grids for ion beam generation. In the present letter, a self-consistent particle-in-cell (PIC) simulation (no a priori assumption on the various energy distributions) is developed to investigate ion-ion plasmas. In particular, it is shown that under the influence of a dc bias, Childlaw type sheaths form within a few microseconds (which corresponds to the ion transit time) and the charged species collected at one of the electrodes is preaccelerated up to the sound speed by a presheath. The consequence of this finding is twofold: the ion velocity at the entrance of the sheath of an ion-ion plasma is larger than the average velocity along one direction of a thermal distribution and, consequently, the sheath size is smaller, which, from an applied perspective, is crucial. Finally, the use of a full PIC simulation allows to

${ }^{a)}$ Electronic mail: meige@lptp.polytechnique.fr. investigate more complex situations (different masses for positive and negative ions, etc.): an example is given at the end of the letter.

The one-dimensional simulation that we have developed is based on the well-known particle-in-cell scheme. ${ }^{17,18}$ The boundaries are absorbing; the left and the right electrodes are biased to the potential $V_{0}>0$ and $-V_{0}$, respectively (the total dc bias is $2 V_{0}$ ). It is assumed that the ion-ion plasma is created outside the simulation and that it enters the simulation box from the top, which comes down to loading particles in the entire volume with a uniform probability profile (including in the sheaths). The initial velocity of each ion loaded into the simulation is chosen randomly from a $300 \mathrm{~K}$ Maxwellian distribution. ${ }^{19}$ The conservative transport of the positive and negative ions is to be investigated; hence, no recombination between positive and negative ions is considered. Finally, the plasma is assumed collisionless.

The results presented in the following are for a 1 -cm-long system represented by $1500-2500$ cells. Simulations are between 10 and $100 \mathrm{~ms}$ long, with time steps between $10^{-10}$ and $10^{-9} \mathrm{~s}$. Up to $2 \times 10^{6}$ macroparticles per species were used. Finally, the properties (mass, injection temperature, and charge) of positive and negative ions were taken equal $\left(m_{+}=m_{-}=40 \mathrm{amu}, T_{+}=T_{-}=300 \mathrm{~K}\right.$, and $\left.q_{+}=-q_{-}=e\right)$.

Figures 1(a) and 1(b) show the steady-state timeaveraged electric potential and the charged species densities across a typical ion-ion plasma simulation, respectively. The dc bias was $2 V_{0}=50 \mathrm{~V}$ and the bulk density was $\sim 10^{16} \mathrm{~m}^{-3}$. The most striking feature observed in Fig. 1(a) is that the plasma potential in the bulk is not the most positive potential of the system (as opposed to electron-ion plasmas) and actually sits halfway between the cathode and the anode potentials. The second interesting feature is the existence of a negatively charged sheath at the anode (left side) and a positively charged sheath at the cathode (the negatively charged sheath edge is shown by the vertical dotted line and was defined as the position where the relative space charge exceeds 10\%). Midha and Economou ${ }^{6}$ and Midha et al. ${ }^{7}$ found similar results using a fluid model but did not investigate the size of the sheath and the existence of a presheath.

The electric potential in the bulk of the ion-ion plasma looks almost perfectly flat [Fig. 1(a)], suggesting that ions enter the sheath with a thermal flux $\Gamma=n \bar{u} / 4=n \overline{\left|u_{x}\right|} / 2$ 

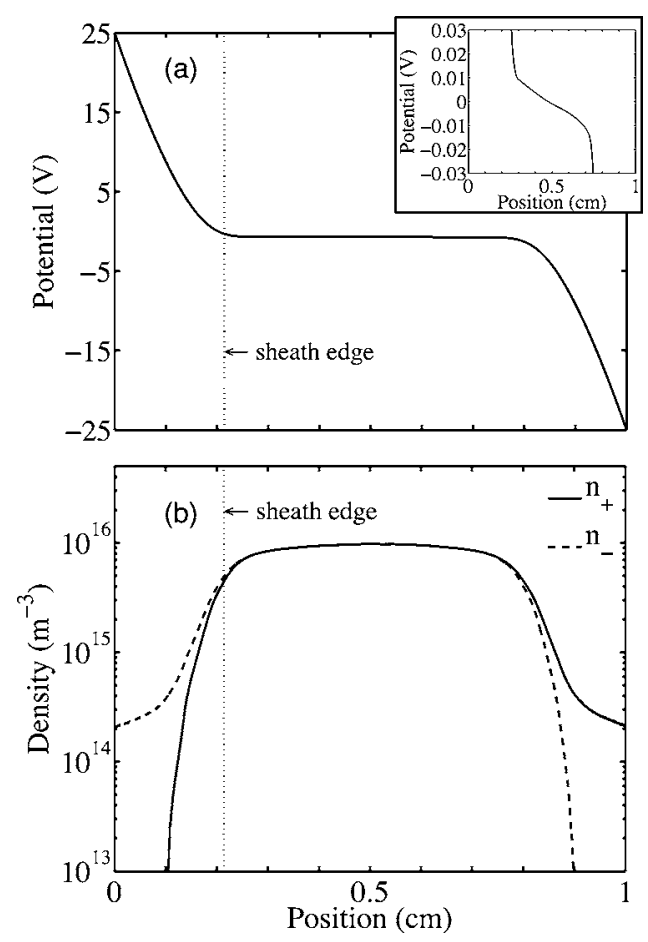

FIG. 1. (a) Electric potential across the plasma, averaged over $10 \mathrm{~ms}$. The inset is a zoom on the same potential and shows the presheath. (b) Positive (solid line) and negative (dashed line) ion densities as a function of position, averaged over $10 \mathrm{~ms}$. The vertical dotted lines in (a) and (b) show the anodic sheath-presheath boundary $(\Delta n / n>10 \%)$.

$=n \sqrt{k_{B} T_{ \pm} / 2 \pi m_{ \pm}}$(where $\bar{u}$ and $\overline{\left|u_{x}\right|}$ are the average velocity and the average along one direction of a Maxwellian distribution, respectively). However, a zoom [inset of Fig. 1(a)] reveals that there exists a small potential drop $(\sim 0.01 \mathrm{~V})$ between the center of the discharge and the sheath edge. The potential drop is of the order of the ion temperature and more precisely around $k_{B} T_{ \pm} / 2 e$. This suggests that, in the same fashion as in electron-ion plasmas, there exists a finite-field region, a presheath, preaccelerating the collected species to a critical velocity before it enters the sheath. Figure 2 shows the average positive (solid line) and negative (dashed line) ion velocities as a function of position. It appears that both positive and negative ions are accelerated to the directed velocity of around $250 \mathrm{~m} / \mathrm{s}$ at the sheath edge (shown by the vertical dotted line). The velocity reached at the sheath edge

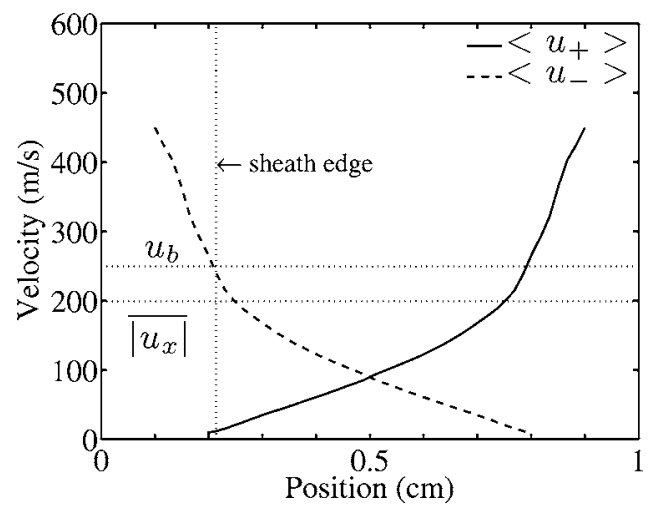

FIG. 2. Average velocity (absolute value) of the positive (solid line) and negative (dashed line) ions as a function of position corresponding to Fig. 1(a). The two horizontal dotted lines represent the Bohm velocity $u_{b}$ and the average velocity $\overline{\mid u_{x}}$ along one direction of a thermal distribution, while the vertical dotted line shows the anodic sheath edge $(\Delta n / n>10 \%)$. Downloaded 03 Apr 2009 to 136.152149 .172 . Redistribution subject

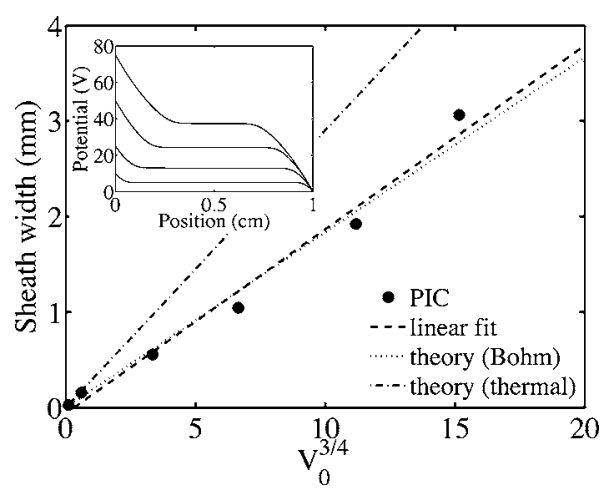

FIG. 3. Sheath width as a function of $V_{0}^{3 / 4}$. The black circles $(-)$ are sheath widths from the simulation and the dashed line is the corresponding linear fit, while the dotted and the dotted-dashed lines are the sheath widths predicted by the theory assuming a Bohm velocity and a thermal velocity, respectively. The inset shows the electric potential across the plasma for various dc biases $2 V_{0}$. Note that for clarity, the potential profiles in the inset were plotted with the right electrode shifted to the same potential reference.

corresponds almost perfectly to $u_{b}=\sqrt{k_{B}\left(T_{+}+T_{-}\right) /\left(m_{+}+m_{-}\right)}$, which is the Bohm velocity where the usual electron temperature was replaced by that of negative ions. This confirms that the collected species does not enter the sheath with a thermal velocity but is preaccelerated by a presheath and that the equivalent of a Bohm criterion exists for ion-ion plasmas.

The width $s$ of the high-voltage sheath in classical electron ion is derived using the positive ion current continuity at the plasma/sheath interface, i.e., by equating the Child-law current to the Bohm current. ${ }^{20}$ The sheath width is then given by

$$
s=\frac{2}{3}\left(\frac{2}{e k_{B}}\right)^{1 / 4}\left(\frac{\epsilon_{0}}{n_{s}}\right)^{1 / 2} \frac{V_{0}^{3 / 4}}{T_{e}^{1 / 4}} .
$$

where $n_{s}$ is the sheath edge density, $V_{0}$ is the applied potential, and $T_{e}$ the electron temperature. Since an equivalent of the Bohm criterion exists in ion-ion plasmas, it can be intuited that the size of the high-voltage sheath in such plasmas follows the same relation as depicted by Eq. (1), with $T_{e}$ replaced by $T_{ \pm}$. The inset in Fig. 3 shows the ion-ion plasma potential profile for various bias potential $V_{0}$ : the sheath width increases with $V_{0}$. Figure 3 shows the sheath width $s$ (where the sheath edge is defined as previously) as a function of $V_{0}^{3 / 4}$ (black dots). These widths can be well fitted by a straight line (dashed line) which the slope is very close (within $5 \%$ ) to the theoretical one given by Eq. (1) and where the electron temperature $T_{e}$ was replaced by the ion temperature $T_{+}$(here, the negative ion sheath is considered). The dotted-dashed line shows the theoretical sheath width if ions were entering the sheath with a thermal velocity rather than with the sound speed (i.e., if there were no presheath). These results show that because of the existence of an equivalent of the Bohm criterion for ion-ion plasmas, the velocity of the ions entering the sheath is larger than just a thermal velocity and the sheath size is, therefore, smaller by a factor of almost 2 .

As mentioned previously, the PIC simulations also allow us to investigate various complex ion-ion plasmas (as opposed to the previous ideal case with equal masses and temperatures); in particular, it is possible to investigate multi-ion plasmas, ion-ion plasmas where positive and negative ions do not have the same mass and temperature, the effect of to AIP license or copyright; see http://apl.aip.org/apl/copyright.jsp 


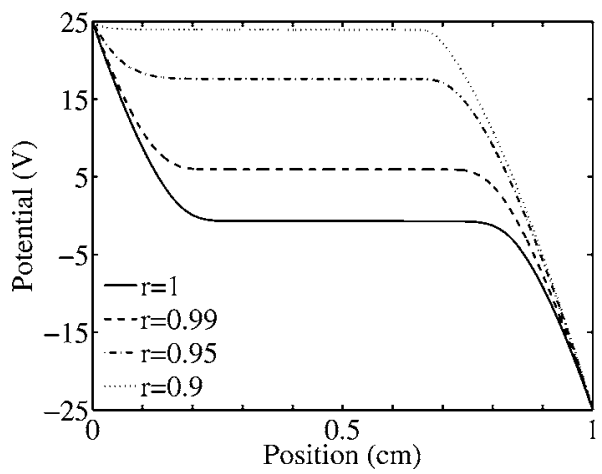

FIG. 4. Electric potential across the plasma, averaged over $10 \mathrm{~ms}$ for various negative to positive ion mass ratios; the solid, dashed, dotted-dashed, and dotted lines are for $r=m_{-} / m_{+}$equal to $1,0.99,0.95$, and 0.9 , respectively.

collisions on the sheath size, the impact of the presence of a small amount of electrons, etc. As an example, Fig. 4 shows the plasma potential profiles for ion-ion plasmas for various positive to negative ion mass ratios. It is observed that even a slight difference in the masses (as small as 5\%) yields a strong asymmetric potential, where the bulk potential sits much closer to one of the electrodes, hence, getting closer to a conventional electron-ion plasma.

In the present letter, we have shown that when a dc voltage is applied across an ion-ion plasma, a presheath appears in order to satisfy the sheath formation conditions: an equivalent of the Bohm criterion (where the usual electron temperature is replaced by that of negative ions) exists. The consequence of this finding is twofold: (i) in ion-ion plasmas, ions entering the sheath are faster than thermal ions; (ii) consequently, the sheath size is smaller by a factor of almost 2. From an applied perspective, these results are crucial as it has been suggested by many authors that ion-ion plasmas could be used in material processing and space propulsion, where the design of extracting grids to create an ion beam is strongly related to the size of the sheaths.

This work was partly supported by ANR (Agence Nationale de la Recherche) under Contract No. ANR-06JCJC-0039.

${ }^{1}$ N. Sato, T. Mieno, T. Hirata, Y. Yagi, R. Hatakeyama, and S. Iizuka, Phys. Plasmas 1, 3480 (1994)

${ }^{2}$ H. Amemiya, Jpn. J. Appl. Phys., Part 1 30, 2601 (1991)

${ }^{3}$ W. Oohara, R. Hatakeyama, and S. Ishiguro, Phys. Rev. E 68, 066407 (2003).

${ }^{4}$ W. Oohara, D. Date, and R. Hatakeyama, Phys. Rev. Lett. 95, 175003 (2005).

${ }^{5}$ V. I. Kolobov and D. J. Economou, Appl. Phys. Lett. 72, 656 (1998).

${ }^{6}$ V. Midha and D. J. Economou, J. Appl. Phys. 90, 1102 (2001).

${ }^{7}$ V. Midha, B. Ramamurthi, and D. J. Economou, J. Appl. Phys. 91, 6282 (2002).

${ }^{8}$ M. Lampe, R. F. Fernsler, S. P. Slinker, S. G. Walton, and D. Leonhardt, 58th Gaseous Electronics Conference, San Jose, CA (American Physical Society, Ridge, New York, 2005).

${ }^{9}$ I. D. Kaganovich, D. J. Economou, B. N. Ramamurthi, and V. Midha, Phys. Rev. Lett. 84, 1918 (2000).

${ }^{10}$ V. Midha and D. J. Economou, Plasma Sources Sci. Technol. 9, 256 (2000).

${ }^{11}$ S. G. Walton, D. Leonhardt, R. F. Fernsler, and R. A. Meger, Appl. Phys. Lett. 81, 987 (2002).

${ }^{12}$ S. G. Walton, D. Leonhardt, R. F. Fernsler, and R. A. Meger, Appl. Phys. Lett. 83, 626 (2003).

${ }^{13}$ W. Oohara and R. Hatakeyama, Phys. Rev. Lett. 91, 205005 (2003).

${ }^{14}$ S. K. Kanakasabapathy, L. J. Overzet, V. Midha, and D. Economou, Appl. Phys. Lett. 78, 22 (2001)

${ }^{15}$ D. J. Economou, Appl. Surf. Sci. 253, 6672 (2007).

${ }^{16}$ P. Chabert, Patent Application No. WO 2007/065915 A1 (pending).

${ }^{17}$ R. W. Hockney and J. W. Eastwood, Computer Simulation Using Particles (IOP, Bristol, 1988)

${ }^{18}$ C. K. Birdsall and A. B. Langdon, Plasma Physics via Computer Simulation (IOP, Bristol, 1991).

${ }^{19}$ K. L. Cartwright, J. P. Verboncoeur, and C. K. Birdsall, J. Comput. Phys. 162, 483 (2000).

${ }^{20}$ M. A. Lieberman and A. J. Lichtenberg, Principles of Plasma Discharges and Materials Processing, 2nd ed. (Wiley Interscience, New York, 2005), p. 177. 



\title{
Fluid model of an electronegative discharge with magnetized electrons and unmagnetized ions
}

\author{
G Leray $^{1}$, P Chabert ${ }^{1}$, A J Lichtenberg ${ }^{2}$ and M A Lieberman ${ }^{2}$ \\ ${ }^{1}$ LPP, CNRS-Ecole Polytechnique, 91128 Palaiseau, France \\ ${ }^{2}$ Department EECS-1770, University of California, Berkeley, CA 94720, USA \\ E-mail: pascal.chabert@lpp.polytechnique.fr
}

Received 10 March 2009, in final form 24 April 2009

Published 18 September 2009

Online at stacks.iop.org/JPhysD/42/194020

\begin{abstract}
A fluid model is developed to determine the cross-field equilibrium densities and fluxes in a finite-size electronegative discharge with magnetized electrons and unmagnetized positive and negative ions. A two-dimensional rectangular geometry with a uniform axial magnetic field is considered. After averaging over the axial losses, the fluid equations, including ion inertia terms, are integrated numerically along the transverse direction to determine the equilibrium quantities, for the boundary condition of vanishing electron density and flux at a transverse insulating wall. This yields equilibrium solutions with equal flows of positive and negative ions to the transverse wall, and with equal positive ion and electron flows to the axial walls. An analytical model is developed for this case, neglecting ion inertia, and is used to determine the scaling of the discharge equilibrium with discharge parameters and to optimize the ion fluxes. The model and the numerical integrations are in reasonable agreement.
\end{abstract}

\section{Introduction}

Ion-ion plasmas are composed of positive and negative ions, with a negligible fraction of electrons $[1,2]$. One potential application is charge-free etching in microelectronics, in which ions of different polarities are alternately accelerated towards the substrate to enhance etching with very little charging effects on the sidewalls $[3,4]$. More recently, it has been proposed to use ion-ion plasmas as the ionization stage of a plasma thruster [5,6]. In such a thruster, ions of opposite signs are accelerated separately to provide thrust, with no need for an additional electron-emitting cathode to neutralize the positive ion beam downstream of the spacecraft.

Ion-ion plasmas can be produced in the afterglow of electronegative (EN) plasmas, or in the periphery of a magnetized EN plasma core. In the first case, electrons are attached to neutrals as they cool after the plasma is switched off. In the second case, electrons are filtered during cross-field transport as their mobility is strongly reduced while negative ions remain unaffected as long as the magnetic field remains moderate.
There have been several experiments showing evidence of ion-ion plasma formation at the periphery of a magnetized EN plasma core [7-10]. A theoretical study of this phenomenon has been given by Franklin and Snell [11]. These authors have used a three fluid model to analyse the cross-field (radial) transport of a one-dimensional (1D) EN plasma infinite in extent along the magnetic field. They chose a transverse boundary condition consistent with a sheath formation (i.e. zero negative ion flux at the radial boundary). As we shall see, an infinite length does not capture essential physics, and the boundary condition is not appropriate if one wants to extract both negative and positive ions at the periphery.

Following the work of Franklin and Snell, we solve the fluid equations in one-dimension (1D) with appropriate boundary conditions at a side wall. Besides small geometric factors, there is little difference between rectangular and cylindrical coordinates, so we work in the simpler rectangular form. As in Franklin's work, we assume that the value of the transverse magnetic field $B_{0}$ is in the range which magnetizes the electrons but leaves the positive and negative ion species unmagnetized. The fluid equations and boundary conditions 
are given in section 2. Besides choosing boundary conditions that are appropriate to our specific design interests, there is a fundamental generalization in our analysis from that of Franklin. Since the electrons are the mobile species along $B_{0}$, the flux to the end walls cannot be neglected. In fact, for the solutions we are seeking, of transversally contained electrons with ion flow to the side walls, it is essential to take the end loss into account. We do this by introducing a loss-parameter $v_{\mathrm{L}}$ that distributes the electron and positive ion end loss, as a volume loss, over the length of the device. For the relatively long-thin configurations of interest, this technique should be adequate. However, there are subtleties in determining the form of $v_{\mathrm{L}}$. We discuss these forms in appendix $\mathrm{A}$, and choose the simplest form for our analysis.

In section 2, we solve the fluid equations looking for the special sheath-free boundary condition at the plasma edge, i.e. contained electrons and, for equal positive and negative ion masses and temperatures, a free flow of the ion species at some fraction of the sound speed to which we assign a parameter $\beta$. This solution requires that the electron density and flux at some $x=R$ ( $R$ is the half-width of the plasma across the magnetic field) satisfy both $n_{\mathrm{e}}(R)=0$ and $\Gamma_{\mathrm{e}}(R)=0$. We illustrate the solutions for a few specific cases, which will then inform approximations that are used to develop an analytic model. The numerical solutions are obtained in terms of the most convenient input parameters, specified by the numerical method, as described in section 2.

In section 3, we formulate the analytic model and obtain solutions for input and output parameters corresponding to the more complete equations used for the numerical solutions. The analytic results can be used to scale the solutions with the inputs at the disposal of the experimentalists, such as $B_{0}$ and the pressure $p$. They also allow an optimization of the desired output, e.g. the transverse ion flux, versus the ratio of on-axis electron density to gas density, $n_{\mathrm{e} 0} / n_{\mathrm{g}}$. As found in the numerics, solutions can be obtained for various velocities $v_{\mathrm{i}}$ of the transversally emerging ions, which can be designated by a parameter $\beta=v_{\mathrm{i}} / u_{\mathrm{Bi}}$, where $u_{\mathrm{Bi}}=\left(e T_{\mathrm{i}} / M_{\mathrm{i}}\right)^{1 / 2}$ is the Bohm (ion loss or sound) speed. Other aspects will be seen from the results and scalings.

In section 4, the numerical and analytic results are compared over the parameter space of initial conditions. This allows us to use the results of the model, which are easily obtained, when they are seen to correspond to the more complete numerical solutions. The reasons for any differences are discussed. Section 5 summarizes our results and discusses assumptions and relations to experiments. The approximations are examined, leading to recommendations for future work. Finally, an assessment of the implications for thrusters is discussed.

\section{Numerical integrations}

The equations to be solved are the first and second moments of the fluid equations for electrons $\left(n_{\mathrm{e}}, v_{\mathrm{e}}\right)$, positive ions $\left(n_{\mathrm{i}}, v_{\mathrm{i}}\right)$ and negative ions $\left(n_{\mathrm{n}}, v_{\mathrm{n}}\right)$, with the $n$ 's and $v$ 's the densities and fluid velocities.

$$
\begin{aligned}
& \nabla\left(n_{\mathrm{e}} v_{\mathrm{e}}\right)=\left(K_{\mathrm{iz}}-K_{\mathrm{att}}\right) n_{\mathrm{g}} n_{\mathrm{e}}-v_{\mathrm{L}} n_{\mathrm{i}}, \\
& \nabla\left(n_{\mathrm{i}} v_{\mathrm{i}}\right)=K_{\mathrm{iz}} n_{\mathrm{g}} n_{\mathrm{e}}-K_{\mathrm{rec}} n_{\mathrm{i}} n_{\mathrm{n}}-v_{\mathrm{L}} n_{\mathrm{i}}, \\
& \nabla\left(n_{\mathrm{n}} v_{\mathrm{n}}\right)=K_{\mathrm{att}} n_{\mathrm{g}} n_{\mathrm{e}}-K_{\mathrm{rec}} n_{\mathrm{i}} n_{\mathrm{n}}, \\
& \boldsymbol{v}_{\mathrm{e}}\left(K_{\mathrm{iz}} n_{\mathrm{g}}+K_{\mathrm{att}} n_{\mathrm{g}}+v_{\mathrm{e}}\right) n_{\mathrm{e}} m-e n_{\mathrm{e}} \nabla \phi+e T_{\mathrm{e}} \nabla n_{\mathrm{e}} \\
& \quad+e n_{\mathrm{e}} \boldsymbol{v}_{\mathrm{e}} \times \boldsymbol{B}_{\mathbf{0}}=0, \\
& \frac{e T_{\mathrm{i}}}{M_{\mathrm{i}}} \nabla n_{\mathrm{i}}+\frac{e n_{\mathrm{i}}}{M_{\mathrm{i}}} \nabla \phi+\nabla\left(n_{\mathrm{i}} v_{\mathrm{i}} v_{\mathrm{i}}\right)+v_{\mathrm{i}} n_{\mathrm{i}} v_{\mathrm{i}}=0, \\
& \frac{e T_{\mathrm{n}}}{M_{\mathrm{n}}} \nabla n_{\mathrm{n}}-\frac{e n_{\mathrm{n}}}{M_{\mathrm{n}}} \nabla \phi+\nabla\left(n_{\mathrm{n}} v_{\mathrm{n}} v_{\mathrm{n}}\right)+v_{\mathrm{n}} n_{\mathrm{n}} v_{\mathrm{n}}=0 .
\end{aligned}
$$

Here $K_{\mathrm{iz}}, K_{\text {att }}$ and $K_{\text {rec }}$ are the reaction rates for the given feedstock gas, $T_{\mathrm{e}}, T_{\mathrm{i}}$ and $T_{\mathrm{n}}$ are the temperatures for the assumed Maxwellian distributions, $m, M_{\mathrm{i}}$ and $M_{\mathrm{n}}$ the masses of the species, and, except for $v_{\mathrm{L}}$, the other quantities have their usual meanings. The loss frequency $v_{\mathrm{L}}$, derived in appendix A, accounts for the end losses in a 1D model for the transverse fluxes:

$$
v_{\mathrm{L}}=\frac{2 D_{\mathrm{i}}}{R L}\left(1+\frac{T_{\mathrm{e}}}{T_{\mathrm{i}}}\right)^{1 / 2},
$$

where $D_{\mathrm{i}}=e T_{\mathrm{i}} / M_{\mathrm{i}} v_{\mathrm{i}}$ is the positive ion diffusion coefficient, $v_{\mathrm{i}}=K_{\mathrm{mi}} n_{\mathrm{g}}$ is the ion-neutral collision frequency with $K_{\mathrm{mi}}$ the ion-neutral momentum transfer rate coefficient and $R L$ is the product of the half-transverse width $R$ and the total length $L$. We have assumed $\omega_{\mathrm{ci}}<v_{\mathrm{i}}$ in $D_{\mathrm{i}}$ as the condition for unmagnetized ions, with $\omega_{\mathrm{ci}}$ the ion gyrofrequency. The inertia terms for the ions, in (5) and (6), although small over most of the transverse dimensions, indicate whether the sound velocity is reached for the ions, when the calculation must be terminated since in the calculations Poisson's equation is replaced by quasi-neutrality

$$
n_{\mathrm{i}}=n_{\mathrm{e}}+n_{\mathrm{n}} .
$$

Equation (4), including the electron magnetization, can be written as

$$
\begin{gathered}
\left(K_{\mathrm{iz}} n_{\mathrm{g}}+K_{\mathrm{att}} n_{\mathrm{g}}+v_{\mathrm{e}}\right) n_{\mathrm{e}} v_{\mathrm{e}}=\frac{e n_{\mathrm{e}}}{m} \nabla \phi-\frac{e T_{\mathrm{e}}}{m} \nabla n_{\mathrm{e}} \\
-n_{\mathrm{e}} v_{\mathrm{e}} \frac{\omega_{\mathrm{ce}}^{2}}{K_{\mathrm{iz}} n_{\mathrm{g}}+K_{\mathrm{att}} n_{\mathrm{g}}+v_{\mathrm{e}}}
\end{gathered}
$$

with $\omega_{\mathrm{ce}}=e B_{0} / m$.

For convenience in obtaining the numerical solutions, we normalize the variables as follows: $X=x / R_{0} ; I, E, N$, $G=\mathrm{n}_{\mathrm{i}, \mathrm{e}, \mathrm{n}, \mathrm{g}} / n_{\mathrm{e} 0} ; c_{\mathrm{s}}=\left(e T_{\mathrm{e}} / M_{\mathrm{i}}\right)^{1 / 2} ; U, V, W=v_{\mathrm{i}, \mathrm{e}, \mathrm{n}} / c_{\mathrm{s}} ;$ $\Phi=-\phi / T_{\mathrm{e}} ; A_{\mathrm{i}, \mathrm{a}}=n_{\mathrm{e} 0} R_{0} K_{\mathrm{iz}, \text { att }} / c_{\mathrm{s}} ; B_{\mathrm{i}}=n_{\mathrm{e} 0} R_{0} K_{\mathrm{rec}} / c_{\mathrm{s}} ;$ $C_{\mathrm{i}, \mathrm{e}, \mathrm{n}}=R_{0} v_{\mathrm{i}, \mathrm{e}, \mathrm{n}} / c_{\mathrm{s}} ; \Omega=R_{0} \omega_{\mathrm{ce}} / c_{\mathrm{s}} ; \varepsilon_{\mathrm{i}, \mathrm{n}}=T_{\mathrm{e}} / T_{\mathrm{i}, \mathrm{n}} ; \zeta=$ $M_{\mathrm{i}} / M_{\mathrm{n}} ; D=R_{0} v_{\mathrm{L}} / c_{\mathrm{s}} ; \Delta=\left(A_{\mathrm{i}}+A_{\mathrm{a}}\right) G+C_{\mathrm{e}} ; \delta=$ $\Delta\left(m / M_{\mathrm{i}}\right)\left(1+\Omega^{2} / \Delta^{2}\right)$. Here, $c_{\mathrm{s}}$ is the usual ambipolar ionsound velocity (in an electropositive plasma), and $R_{0}$ is a conveniently chosen normalization length, e.g. $R_{0}=2.5 \mathrm{~cm}$, which might correspond to an experimental radius [6], but only the quantity $R$ has a physical meaning in the theory. 
With these normalizations, the normalized equations are

$$
\begin{aligned}
& \frac{\mathrm{d} E V}{\mathrm{~d} X}=\left(A_{\mathrm{i}}-A_{\mathrm{a}}\right) G E-D I, \\
& \frac{\mathrm{d} I U}{\mathrm{~d} X}=A_{\mathrm{i}} G E-B_{\mathrm{i}} I N-D I, \\
& \frac{\mathrm{d} N W}{\mathrm{~d} X}=A_{\mathrm{a}} G E-B_{\mathrm{i}} I N, \\
& \frac{\mathrm{d} E}{\mathrm{~d} X}+E \frac{\mathrm{d} \Phi}{\mathrm{d} X}+\delta E V=0, \\
& \frac{1}{\varepsilon_{\mathrm{i}}} \frac{\mathrm{d} I}{\mathrm{~d} X}-I \frac{\mathrm{d} \Phi}{\mathrm{d} X}+\frac{\mathrm{d} I U^{2}}{\mathrm{~d} X}+C_{\mathrm{i}} I U=0, \\
& \frac{\zeta}{\varepsilon_{\mathrm{n}}} \frac{\mathrm{d} N}{\mathrm{~d} X}+\zeta N \frac{\mathrm{d} \Phi}{\mathrm{d} X}+\frac{\mathrm{d} N W^{2}}{\mathrm{~d} X}+C_{\mathrm{n}} N W=0 .
\end{aligned}
$$

Using quasi-neutrality, $I=E+N$, to eliminate $I$, we then have six equations to determine the six variables, which in matrix form, with

$$
Y=[E N U V W \Phi]^{\mathrm{T}},
$$

can be written as

$$
M \times Y^{\prime}=R H S,
$$

with

$$
M=\left[\begin{array}{cccccc}
V & 0 & 0 & E & 0 & 0 \\
U & U & I & 0 & 0 & 0 \\
0 & W & 0 & 0 & N & 0 \\
1 & 0 & 0 & 0 & 0 & E \\
\varepsilon_{\mathrm{i}}^{-1}+U^{2} & \varepsilon_{\mathrm{i}}^{-1}+U^{2} & 2 I N & 0 & 0 & -I \\
0 & \zeta / \varepsilon_{\mathrm{n}}+W^{2} & 0 & 0 & 2 N W & \zeta N
\end{array}\right]
$$

and

$$
R H S=\left[\begin{array}{l}
\left(A_{\mathrm{i}}-A_{\mathrm{a}}\right) G E-D I \\
A_{\mathrm{i}} G E-B_{\mathrm{i}} I N-D I \\
A_{\mathrm{a}} G E-B_{\mathrm{i}} I N \\
-\delta E V \\
-C_{\mathrm{i}} I U \\
-C_{\mathrm{n}} N W
\end{array}\right]
$$

The integration is performed using

$$
Y^{\prime}=M^{-1} \times R H S .
$$

The transverse wall is assumed to be insulating and with a potential independent of the grounded ends. Although not the only possible type of solution, we choose to examine a solution set in which, at some $x=R$

$$
\begin{aligned}
& n_{\mathrm{e}}(R)=0, \\
& \Gamma_{\mathrm{e}}(R)=0 .
\end{aligned}
$$

At this $R$, the ion flux and density (the same for both species) are determined, with the condition that the ion drift velocity lies between zero and the ion Bohm velocity $u_{\mathrm{Bi}}\left(\Gamma_{\mathrm{i}}=\beta n_{\mathrm{i}} u_{\mathrm{Bi}}\right.$ with $0<\beta<1)$. For these boundary conditions, we
Table 1. Reaction rates in $\mathrm{m}^{3} \mathrm{~s}^{-1}$ and collision frequencies in $\mathrm{s}^{-1}$ for an oxygen-like feedstock gas, except that, for simplicity, the ion collision frequencies are taken to be equal, and the oxygen molecular mass is used for both species.

\begin{tabular}{ll}
\hline Parameter & Value \\
\hline$K_{\text {iz }}$ & $2.34 \times 10^{-15} T_{\mathrm{e}}^{1.03} \exp \left(-12.29 / T_{\mathrm{e}}\right)$ \\
$K_{\text {att }}$ & $1.07 \times 10^{-15} T_{\mathrm{e}}^{-1.391} \exp \left(-6.26 / T_{\mathrm{e}}\right)$ \\
$K_{\text {rec }}$ & $5.2 \times 10^{-14}\left(0.026 / T_{\mathrm{i}}\right)^{0.44}$ \\
$\nu_{\mathrm{i}}$ & $3.95 \times 10^{-16} n_{\mathrm{g}}$ \\
$v_{\mathrm{e}}$ & $4.7 \times 10^{-14} T_{\mathrm{e}}^{1 / 2} n_{\mathrm{g}}$ \\
$v_{\mathrm{n}}$ & $3.95 \times 10^{-16} n_{\mathrm{g}}$ \\
\hline
\end{tabular}

expect solutions to exist only when $D_{\perp \mathrm{e}}<D_{\mathrm{i}}$, which sets the condition for magnetized electrons. Beyond this position, there is either an insulating wall or a pure positive ion-negative ion plasma. These solutions are special in that they allow the extraction of both positive and negative ions at a surface which has no sheath (see section 5 for further discussion).

The method for the numerical solution is to first choose $n_{\mathrm{e} 0}$ and $\alpha_{0}$, where $n_{\mathrm{e} 0}$ is the centre $x=0$ (on-axis if radial coordinates were used) electron density and $\alpha_{0}=n_{\mathrm{n} 0} / n_{\mathrm{e} 0}$ is the EN ratio at $x=0$. For convenience, we take $T_{\mathrm{i}}=T_{\mathrm{n}}$, $M_{\mathrm{i}}=M_{\mathrm{n}}$ and a constant and determined ratio $T_{\mathrm{e}} / T_{\mathrm{i}}$. We then vary $T_{\mathrm{e}}$ until (21) and (22) are satisfied at some $R$. Since, from (7), $v_{\mathrm{L}}$ is determined by the choice of a specific $R L$, this also determines the length $L$. As we shall see in sections 3 and 4 , after the solutions in the $n_{\mathrm{e} 0}$ versus $\alpha_{0}$ parameter space are determined, the curves of constant values of $R$ and $L$ are obtained, corresponding to a set of device dimensions. We note that $\alpha_{0}$ is in fact determined from the device dimensions and $n_{\mathrm{e} 0}$ is determined from the input power. The solution set for a fixed value of $R L$ then determines the range of $\alpha_{0}$ values for the permissible set of $n_{\mathrm{e} 0}$ values. As we shall see, in sections 3 and 4 , scanning $\alpha_{0}$ at constant $n_{\mathrm{e} 0}$, or $n_{\mathrm{e} 0}$ at constant $\alpha_{0}$, we find a set of solutions (at various values of $R, L$ and $T_{\mathrm{e}}$ ) that span a range of exiting fluxes from

$$
\Gamma_{\mathrm{i}}(R)=0
$$

to

$$
\beta \equiv \frac{\Gamma_{\mathrm{i}}(R)}{n_{\mathrm{i}}(R) u_{\mathrm{Bi}}}=1
$$

with $\Gamma_{\mathrm{i}}=\Gamma_{\mathrm{n}}$ and $u_{\mathrm{Bi}}=\left(e T_{\mathrm{i}} / M_{\mathrm{i}}\right)^{1 / 2}$. We note that (23) corresponds to a volume balance of attachment and recombination, while (24) corresponds to the ion-sound limit where quasi-neutrality is violated. For physical applications, we are interested in solutions near the limit (24) for which we are extracting ions. For a given device with a fixed $R$ and $L$, which specifies $R L$, the locus of constant $R$ in the parameter space of $n_{\mathrm{e} 0}$ versus $\alpha_{0}$ gives a desired solution set.

To understand the above in more detail, we choose a particular example corresponding, roughly, to an oxygen feedstock gas with parameters given in table 1 , as is used in an ongoing experiment [6]. The rate coefficients $K_{\mathrm{iz}}, K_{\mathrm{att}}, K_{\text {rec }}$, and $K_{\mathrm{me}}$ are from [12, table 8.2] reactions 1, 2, 4, 7 and 9, and the rate coefficient $K_{\mathrm{mi}}$ is from [12, p 366]. We consider a pressure $p=10$ mTorr, a magnetic field $B_{0}=300 \mathrm{G}$, which corresponds to magnetized electrons and unmagnetized 

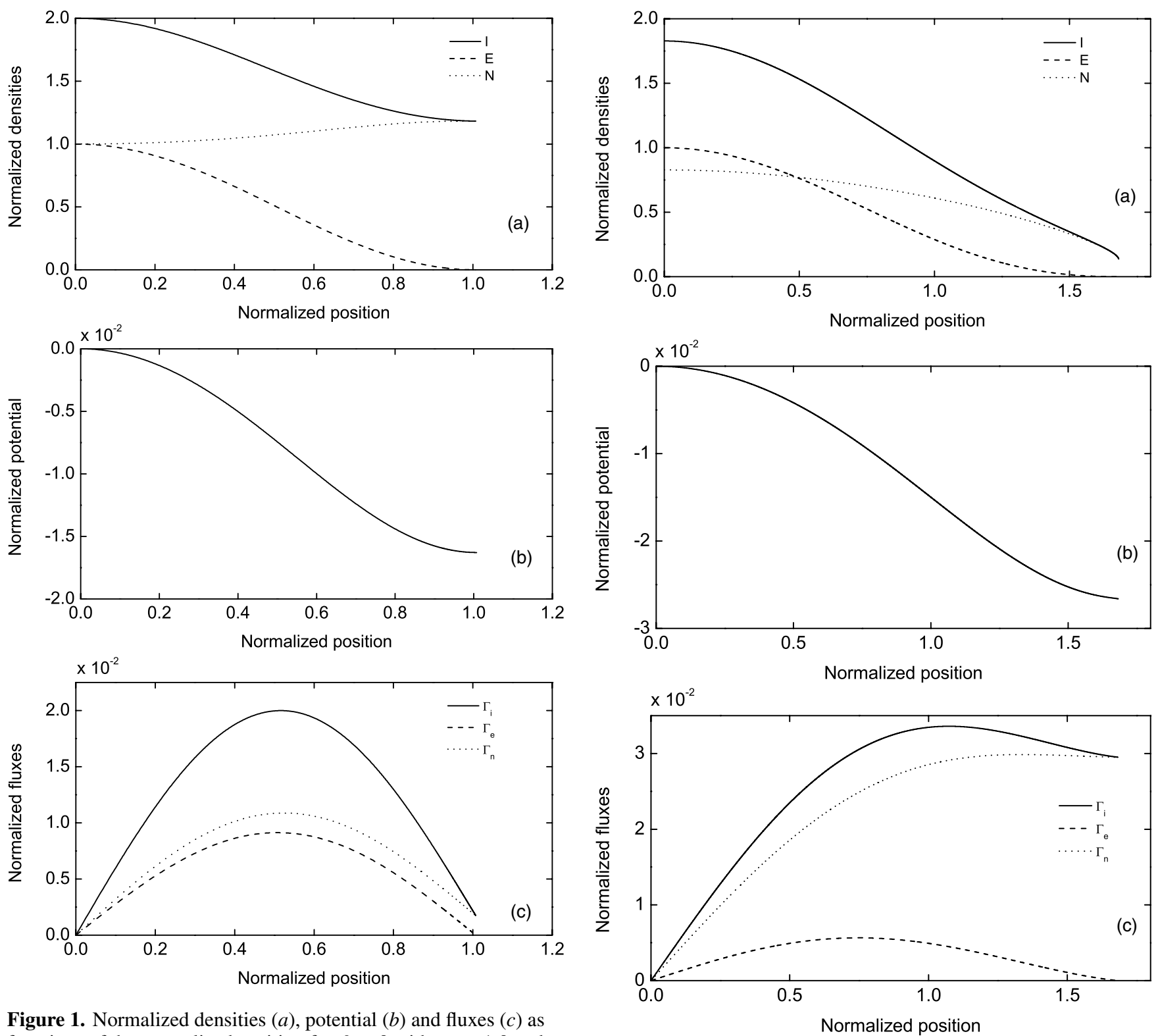

Figure 1. Normalized densities (a), potential $(b)$ and fluxes $(c)$ as functions of the normalized position for $\beta=0$ with $\alpha_{0}=1.0$ and $n_{\mathrm{e} 0} / n_{\mathrm{g}}=2.75 \times 10^{-4}$. Normalizations are $X=x / R_{0}$;

$I, E, N=n_{\mathrm{i}, \mathrm{e}, \mathrm{n}} / n_{\mathrm{e} 0} ; \Gamma_{\mathrm{i}, \mathrm{e}, \mathrm{n}}=n_{\mathrm{i}, \mathrm{e}, \mathrm{n}} v_{\mathrm{i}, \mathrm{e}, \mathrm{n}} /\left(n_{\mathrm{e} 0} c_{\mathrm{s}}\right) ; \Phi=-\phi / T_{\mathrm{e}}$.

ions, and we use the nominal value of $T_{\mathrm{e}} / T_{\mathrm{i}}=20$. A value of $R L=100 \mathrm{~cm}^{2}$ is chosen to roughly correspond to an experimental configuration [6], with a half-width $R=3 \mathrm{~cm}$ and a length $L=33 \mathrm{~cm}$ (within the analysis, it could well apply to a configuration with, e.g. $R=2.5 \mathrm{~cm}$ and $L=40 \mathrm{~cm}$, which we shall illustrate). Solving as described above, for a fixed $n_{\mathrm{e} 0} / n_{\mathrm{g}}=2.75 \times 10^{-4}$, we obtain results for two characteristic values of $\alpha_{0}$ as given in figures 1 and 2. In figure 1, we take $\alpha_{0}=1.0$, which is close to the limiting value of $\Gamma_{\mathrm{i}}(R)=0$. In figure 2, we take $\alpha_{0}=0.828$ which gives a finite value of $\Gamma_{\mathrm{i}}(R)$ near $\beta=1$. In both figures the electron density and flux goes to zero at the radial boundary, as required by the boundary conditions (21) and (22). Because of quasi-neutrality, the positive ion density equals the negative ion density at $x=R$. Similarly, the ion fluxes become equal at $x=R$ because $\Gamma_{\mathrm{i}}=\Gamma_{\mathrm{e}}+\Gamma_{\mathrm{n}}$ from (1), (2) and (3). The profile of the potential indicates that the electric field vanishes at $x=R$, confirming

Figure 2. Normalized densities $(a)$, potential $(b)$ and fluxes $(c)$ as functions of the normalized position for $\beta=1$ with $\alpha_{0}=0.828$ and $n_{\mathrm{e} 0} / n_{\mathrm{g}}=2.75 \times 10^{-4}$. Normalizations are $X=x / R_{0}$;

$I, E, N=n_{\mathrm{i}, \mathrm{e}, \mathrm{n}} / n_{\mathrm{e} 0} ; \Gamma_{\mathrm{i}, \mathrm{e}, \mathrm{n}}=n_{\mathrm{i}, \mathrm{e}, \mathrm{n}} v_{\mathrm{i}, \mathrm{e}, \mathrm{n}} /\left(n_{\mathrm{e} 0} c_{\mathrm{s}}\right) ; \Phi=-\phi / T_{\mathrm{e}}$.

that there is no sheath and that the ions may be extracted. We see in figure 1 that the negative ion density is relatively constant while the fluxes pass through a maximum at about half the simulation length. In figure 2 , the negative ion density decays towards the edge, while both ion fluxes increase, reaching a plateau near the edge. Finally, we note in both figures that $n_{\mathrm{e}}(x) / n_{\mathrm{e} 0}$ has a form similar to $1+\cos (\pi x / R)$. We use all these observations to develop an analytic model in section 3 that gives approximate scalings with parameters. We compare the analytic model and numerical simulations in section 4 .

In figure 3 we show the numerical solutions for $n_{\mathrm{e} 0} / n_{\mathrm{g}}$ versus $\alpha_{0}$, with $\beta$ as a parameter, for $p=10 \mathrm{mTorr}$ and $R L=100 \mathrm{~cm}^{2}$. Values of $\beta \approx 0,0.15$ and 1 are shown. To construct the $\beta$-curves, a fixed value of $n_{\mathrm{e} 0}$ (or $\alpha_{0}$ ) is first chosen. Next, a sequence of values of $\alpha_{0}$ (or $n_{\mathrm{e} 0}$ ) is chosen, 


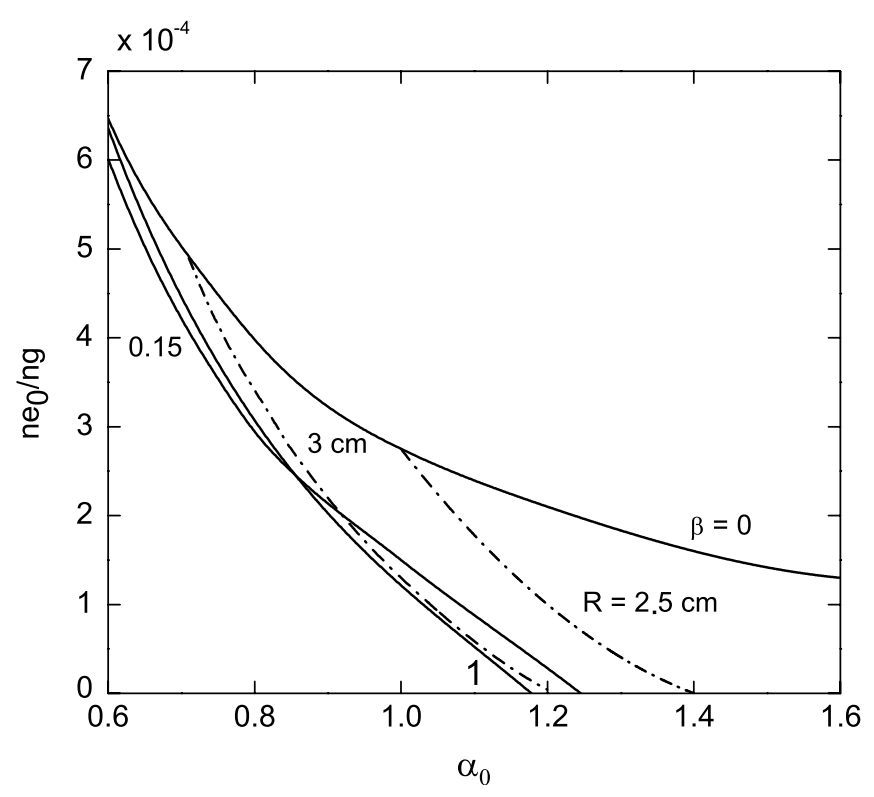

Figure 3. $n_{\mathrm{e} 0} / n_{\mathrm{g}}$ versus $\alpha_{0}$ for $p=10 \mathrm{mTorr}, B_{0}=300 \mathrm{G}$, $R L=100 \mathrm{~cm}^{2}$, and $T_{\mathrm{e}} / T_{\mathrm{i}}=20$. The upper-through-lower solid curves are for $\beta=0,0.15$ and 1, respectively; the dash-dot curves give $R=3 \mathrm{~cm}$ and $R=2.5 \mathrm{~cm}$, which are the solutions for two fixed device configurations.

corresponding to a horizontal (or vertical) scan in the figure. Finally, for each value of $n_{\mathrm{e} 0}$ and $\alpha_{0}$ along this horizontal (or vertical) line, a set of values of $T_{\mathrm{e}}$ are investigated. For each $T_{\mathrm{e}}$, the equations are integrated to the point where $\Gamma_{\mathrm{e}}(R) \approx 0$. If a value of $T_{\mathrm{e}}$ also makes $n_{\mathrm{e}}(R) \approx 0$, then a solution has been found and the value of $\beta$ is calculated. The approximate bounding values of $\beta=0$ and $\beta=1$ are shown as solid lines. The $\beta=1$ line cannot be obtained exactly due to the inertia-caused singularity, and is therefore represented by points with $\beta$ close to 1 . Numerically, it was found that the $\beta$-solutions can intersect for different values of $\beta$. For example, the $\beta \approx 0.15$ curve intersects the $\beta \approx 1$ curve at $\alpha_{0} \approx 0.86$ and $n_{\mathrm{e} 0} / n_{\mathrm{g}} \approx 2.4 \times 10^{-4}$. The $T_{\mathrm{e}}$ 's for these solutions at the intersection are, of course, different; i.e. $T_{\mathrm{e}} \approx 2.49 \mathrm{eV}$ at $\beta \approx 1$ is lower than $T_{\mathrm{e}} \approx 2.52 \mathrm{eV}$ at $\beta \approx 0.15$. Curves of constant $R=3 \mathrm{~cm}(L=33.3 \mathrm{~cm})$ and constant $R=2.5 \mathrm{~cm}(L=40 \mathrm{~cm})$ are shown as dash-dot lines. This figure shows that for a given electronegativity $\alpha_{0}$, there is a specific range of ionization fraction values $\left(n_{\mathrm{e} 0} / n g\right)$ that yields the wanted solutions. The region to the left of the $\beta \approx 1$ is a region where $\beta$ would need to be greater than 1 for us to find solutions following the boundary conditions. No solutions can therefore be found. The region to the right of the $\beta \approx 0$ curve corresponds to negative $\beta$ values, meaning that an input of ion fluxes is needed to sustain the plasma. These solutions are not what we are looking for. The region between $\beta \approx 0$ and $\beta \approx 1$ is where all the self-sustained solutions fulfilling the boundary conditions can be found.

Because of the way the boundary conditions have been chosen, and the way the end loss term has been approximated, it has not been necessary to specify whether the side and end walls are conducting or insulating. For an experiment, choices must be made, and the side and end walls cannot both be conducting. The reason is that electrons are free to move along the magnetic field such that the plasma must be sufficiently positive with respect to the ends to confine electrons. In experiments, and also in simulations, the side walls are insulated, and thus can have potentials close to that of the plasma. The ends can be either conducting or insulating.

\section{Analytical model}

As in the numerical solutions of the differential equations for the positive ion, negative ion and electron velocities and fluxes, we consider a two dimensional rectangular discharge of axial length $L$ along $z$ and transverse width ('diameter') $2 R$ along $x$, with a constant axial magnetic field $B_{0}$, and with $y$ an ignorable coordinate. As in the numerical solutions, fixed values of $B_{0}$, $R L$ and $T_{\mathrm{e}} / T_{\mathrm{i}}$ are chosen. The ion inertia effects that are included in the numerics are neglected in the analytical model.

We initially specify the solution in terms of the spatially averaged electronegativity, $\bar{\alpha}=\bar{n}_{\mathrm{n}} / n_{\mathrm{e} 0}$. Later, we relate $\bar{\alpha}$ to the central electronegativity $\alpha_{0}$ which is specified in the numerics. Assuming electron inertia to be negligible, the driftdiffusion equation for the transverse electron flux is

$$
\Gamma_{\perp \mathrm{e}}=-D_{\perp \mathrm{e}} \frac{\mathrm{d} n_{\mathrm{e}}}{\mathrm{d} x}-\mu_{\perp \mathrm{e}} n_{\mathrm{e}} E
$$

where

$$
D_{\perp \mathrm{e}}=\frac{D_{\mathrm{e}}}{1+\omega_{\mathrm{ce}}^{2} / \nu_{\mathrm{e}}^{2}}, \quad \mu_{\perp \mathrm{e}}=\frac{\mu_{\mathrm{e}}}{1+\omega_{\mathrm{ce}}^{2} / \nu_{\mathrm{e}}^{2}}
$$

with

$$
D_{\mathrm{e}}=\frac{e T_{\mathrm{e}}}{m \nu_{\mathrm{e}}}, \quad \mu_{\mathrm{e}}=\frac{e}{m v_{\mathrm{e}}}
$$

the unmagnetized electron diffusion and mobility coefficients, $v_{\mathrm{e}}=K_{\mathrm{me}} n_{\mathrm{g}}$ the electron-neutral collision frequency, $K_{\mathrm{me}}$ the electron-neutral momentum transfer rate coefficient and $n_{\mathrm{g}}$ the gas density. We assume a magnetic field sufficiently large that the transverse electric field $E \sim T_{\mathrm{i}} / R$, and we estimate $\mathrm{d} n_{\mathrm{e}} / \mathrm{d} x \sim n_{\mathrm{e} 0} / R$. Since $T_{\mathrm{i}} \ll T_{\mathrm{e}}$, the drift term is small compared with the diffusion term, and (25) reduces to

$$
\Gamma_{\perp \mathrm{e}} \approx-D_{\perp \mathrm{e}} \frac{\mathrm{d} n_{\mathrm{e}}}{\mathrm{d} x}
$$

For $L \gg R$, we can take the electron density to be fairly uniform over most of the $z$-direction, which allows us to incorporate the axial losses in the electron balance equation in $x$ as

$$
-D_{\perp \mathrm{e}} \frac{\mathrm{d}^{2} n_{\mathrm{e}}}{\mathrm{d} x^{2}}=\left(v_{\mathrm{iz}}-v_{\text {att }}\right) n_{\mathrm{e}}-v_{\mathrm{L}}\left(n_{\mathrm{e}}+n_{\mathrm{n}}\right),
$$

where $v_{\mathrm{iz}}$ is the ionization frequency, $v_{\text {att }}$ is the attachment frequency, and $v_{\mathrm{L}}$ is a heuristic axial loss frequency (see appendix A). Here $n_{\mathrm{n}}$ is the negative ion density, and we note that the axial electron loss is proportional to the positive ion density, which is the sum of the electron and negative ion densities. Therefore, the right hand side of (29) can change sign (from positive to negative) if $n_{\mathrm{e}} \rightarrow 0$ at the transverse edge 
$x=R$. This yields the numerically found desired solution for $n_{\mathrm{e}}(x)$, which has a negative curvature near $x=0$ and a positive curvature near the edge; i.e. both $n_{\mathrm{e}}$ and $\Gamma_{\perp \mathrm{e}}$ can approach zero at $x=R$, terminating the electron processes.

For a specified variation of $n_{\mathrm{n}}(x)$, we can solve (29) for $n_{\mathrm{e}}(x)$. We use the approximation $n_{\mathrm{n}} \approx \bar{n}_{\mathrm{n}}=$ const to obtain a solution, where $\bar{n}_{\mathrm{n}}$ is the spatially averaged negative ion density. We use the set of boundary conditions

$$
\begin{gathered}
\frac{\mathrm{d} n_{\mathrm{e}}}{\mathrm{d} x}=0 \text { at } x=0, \quad \frac{\mathrm{d} n_{\mathrm{e}}}{\mathrm{d} x}=0 \text { at } x=R, \\
n_{\mathrm{e}}=0 \text { at } x=R
\end{gathered}
$$

as described earlier for the numerics. The solution of (29) that satisfies the boundary conditions (30) is

$$
n_{\mathrm{e}}(x)=\frac{n_{\mathrm{e} 0}}{2}\left(1+\cos \frac{\pi x}{R}\right) .
$$

Defining

$$
v_{\text {net }}=v_{\text {iz }}-v_{\text {att }}-v_{\mathrm{L}}
$$

to be the net transverse electron production frequency after subtracting the attachment and axial losses, then the boundary conditions require

$$
n_{\mathrm{e} 0}=2 \bar{n}_{\mathrm{n}} \frac{\nu_{\mathrm{L}}}{v_{\text {net }}}
$$

and

$$
\frac{\pi^{2}}{R^{2}}=\frac{v_{\text {net }}}{D_{\perp \mathrm{e}}} .
$$

Relation (33) can be written as

$$
v_{\text {net }}=2 \bar{\alpha} v_{\mathrm{L}},
$$

where $\bar{\alpha}=\bar{n}_{\mathrm{n}} / n_{\mathrm{e} 0}$ is the average negative ion density normalized to $n_{\mathrm{e} 0}$.

Approximate expressions for the axial loss frequency $v_{\mathrm{L}}$ are described in appendix A. We use expression (A.8) given in the appendix [see also (7)]. It is convenient to choose a fixed value $R L=$ const in the numerical work, so we also choose this condition in the analytical model.

Substituting (35) into (34) and using (7), we obtain

$$
\bar{\alpha}=\frac{\pi^{2}}{4 R^{2}} \frac{D_{\perp \mathrm{e}}}{D_{\mathrm{i}}} \frac{R L}{\left(1+T_{\mathrm{e}} / T_{\mathrm{i}}\right)^{1 / 2}} .
$$

Substituting (35) into (32) and using (7), and (36), we obtain

$$
\frac{2 D_{\mathrm{i}}(1+2 \bar{\alpha})}{R L}\left(1+\frac{T_{\mathrm{e}}}{T_{\mathrm{i}}}\right)^{1 / 2}=\left(K_{\mathrm{iz}}-K_{\mathrm{att}}\right) n_{\mathrm{g}},
$$

where we have used $v_{\text {iz }}=K_{\text {iz }} n_{\mathrm{g}}$ and $\nu_{\text {att }}=K_{\text {att }} n_{\mathrm{g}}$, with $K_{\text {iz }}$ and $K_{\text {att }}$ the ionization and attachment rate coefficients (functions of $T_{\mathrm{e}}$ alone). Equation (37) can be solved to obtain $T_{\mathrm{e}}$ for specified values of $n_{\mathrm{g}}, B_{0}, R L, T_{\mathrm{e}} / T_{\mathrm{i}}$ and $\bar{\alpha}$. Then (36) can be solved to obtain the discharge radius $R$, with $L$ then found from the relation $R L=$ const.

To complete the description of the discharge equilibrium, we use an approximate negative ion balance relation

$$
\frac{\mathrm{d} \Gamma_{\mathrm{n}}}{\mathrm{d} x}=v_{\mathrm{att}} n_{\mathrm{e}}(x)-K_{\mathrm{rec}} \bar{n}_{\mathrm{n}}\left(\bar{n}_{\mathrm{n}}+n_{\mathrm{e}}(x)\right) .
$$

where $\Gamma_{\mathrm{n}}$ is the negative ion flux. Substituting (31) for $n_{\mathrm{e}}(x)$ into (38) and with the boundary condition that $\Gamma_{\mathrm{n}}(0)=0$, we integrate (38) to obtain

$\Gamma_{\mathrm{n}}(x)=\frac{1}{2} n_{\mathrm{e} 0}\left(v_{\mathrm{att}}-K_{\mathrm{rec}} \bar{n}_{\mathrm{n}}\right)\left(x+\frac{R}{\pi} \sin \frac{\pi x}{R}\right)-K_{\mathrm{rec}} \bar{n}_{\mathrm{n}}^{2} x$.

Equating the flux at $x=R$ to be proportional to a Bohm edge flux, we obtain

$\Gamma_{\mathrm{n}}(R)=n_{\mathrm{e} 0}\left[\frac{1}{2} v_{\mathrm{att}}-K_{\mathrm{rec}} n_{\mathrm{e} 0}\left(\frac{1}{2} \bar{\alpha}+\bar{\alpha}^{2}\right)\right] R=\beta n_{\mathrm{e} 0} \alpha_{\mathrm{s}} u_{\mathrm{Bi}}$,

where $\beta=v_{\mathrm{i}} / u_{\mathrm{Bi}}$ is the transverse ion velocity ratio, $\alpha_{\mathrm{s}}=$ $n_{\mathrm{n}}(R) / n_{\mathrm{e} 0}$ is the negative ion density at the transverse edge, normalized to $n_{\mathrm{e} 0}$, and $u_{\mathrm{Bi}}=\left(e T_{\mathrm{i}} / M_{\mathrm{i}}\right)^{1 / 2}$ is the characteristic ion loss velocity. The factors of $\frac{1}{2}$ in (39) and (40) arise from averaging the spatially varying electron density over the volume. The first and second terms in square brackets in (40) give the frequency of negative ion generation and the recombination loss frequency, respectively; from the right hand side, the transverse loss frequency is $\beta \alpha_{\mathrm{s}} u_{\mathrm{Bi}} / R$. The range of $\beta$ for a physical solution of the type that we seek to exist is $0 \leqslant \beta \leqslant 1$; i.e. the ion flux cannot become negative or exceed the flux at sound speed.

The relation between centre and edge negative ion densities is found using a drift-diffusion approximation for the negative ion flux

$$
\Gamma_{\mathrm{n}}(x)=-D_{\mathrm{i}} \frac{\mathrm{d} n_{\mathrm{n}}}{\mathrm{d} x}-\mu_{\mathrm{i}} n_{\mathrm{n}} E
$$

with $D_{\mathrm{i}}$ and $\mu_{\mathrm{i}}$ the negative ion diffusion and mobility coefficients, assumed in this model to be the same for positive and negative ions

$$
D_{\mathrm{i}}=\frac{e T_{\mathrm{i}}}{M_{\mathrm{i}} v_{\mathrm{n}}}, \quad \mu_{\mathrm{i}}=\frac{e}{M_{\mathrm{i}} v_{\mathrm{n}}}
$$

and with $E$ the ambipolar electric field. Equating (41) to (39) and using a self-consistent expression for the ambipolar field, we obtain the relation between $\bar{\alpha}$ and $\alpha_{\mathrm{s}}$. The calculation is complicated and is given in appendix $\mathrm{B}$. The result is

$$
\begin{aligned}
\alpha_{\mathrm{s}}\left[\frac{3 D_{\mathrm{i}}}{2}+\frac{\beta u_{\mathrm{Bi}} R}{2}+\frac{2 \beta u_{\mathrm{Bi}} R}{\pi^{2}(1+2 \bar{\alpha})}\right] \\
=\bar{\alpha}\left[\frac{3 D_{\mathrm{i}}}{2}+\frac{D_{\mathrm{i}}-D_{\perp \mathrm{e}}}{1+\mu_{\perp \mathrm{e}} / \mu_{\mathrm{i}}} \ln \left(1+\frac{1+\mu_{\perp \mathrm{e}} / \mu_{\mathrm{i}}}{2 \bar{\alpha}}\right)\right. \\
\left.-\frac{2 v_{\mathrm{att}}}{1+2 \bar{\alpha}} \frac{R^{2}}{\pi^{2}}\right] .
\end{aligned}
$$

For a specified value of $\beta$, substituting $\alpha_{\mathrm{s}}$ from (43) into the right hand equality in (40), we obtain $n_{\mathrm{e} 0}$ as a function of $\bar{\alpha}$. The solutions for $R$ and $n_{\mathrm{e} 0}$ are relatively insensitive to $T_{\mathrm{e}}$, as also found numerically, so a nominal value, e.g. $T_{\mathrm{e}}=2.5 \mathrm{~V}$, can be used to determine the scalings. For sufficiently high magnetic fields $\omega_{\text {ce }} / \nu_{\mathrm{e}} \gg 1$, with $R L=$ const, we find the 


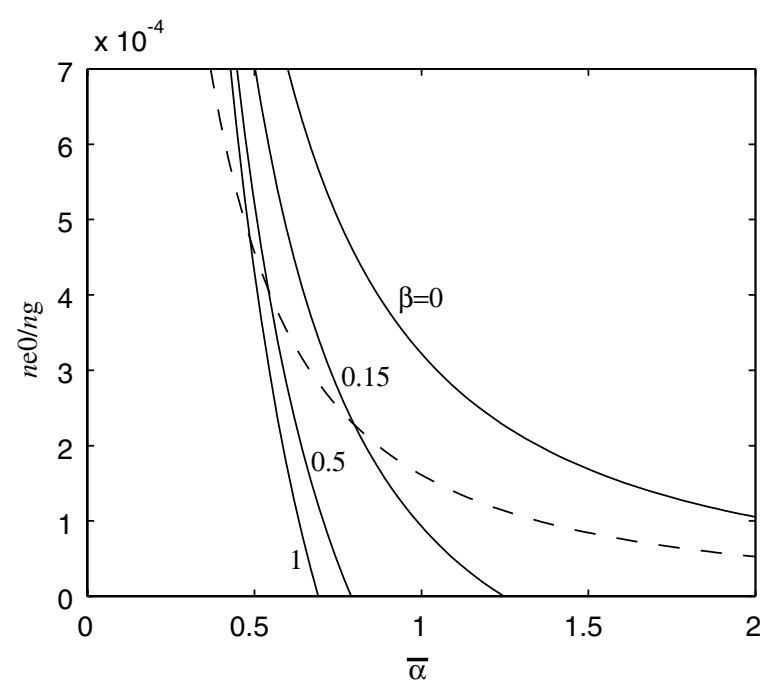

Figure 4. $n_{\mathrm{e} 0} / n_{\mathrm{g}}$ versus $\bar{\alpha}$ for $p=10 \mathrm{mTorr}, B_{0}=300 \mathrm{G}$,

$R L=100 \mathrm{~cm}^{2}$, and $T_{\mathrm{e}} / T_{\mathrm{i}}=20$. The upper-through-lower solid curves are for $\beta=0,0.15,0.5$ and 1, respectively; the dashed curve gives $n_{\mathrm{e} 0}(\mathrm{opt}) / n_{\mathrm{g}}$.

scaling for $R$ from (36)

$$
R \propto \frac{n_{\mathrm{g}}}{B_{0} \bar{\alpha}^{1 / 2}}
$$

or at fixed $B_{0}, n_{\mathrm{g}}$ and $R$, we find that $\bar{\alpha}$ is independent of $n_{\mathrm{e} 0}$.

An 'optimum' choice of $n_{\mathrm{e} 0}$ at a given $\bar{\alpha}$ can be found by maximizing the transverse ion flux $\Gamma_{\mathrm{n}}$ at $x=R$, at a fixed $R$, $L$ and $n_{\mathrm{g}}$. Physically, this is because the attachment scales as the density while the recombination loss scales as the square of the density. Maximizing $\Gamma_{\mathrm{n}}(R)$ with respect to $n_{\mathrm{e} 0}($ at fixed $\bar{\alpha})$, using the first equality in (40), we obtain

$$
n_{\mathrm{e} 0}(\mathrm{opt})=\frac{v_{\mathrm{att}}}{4 K_{\mathrm{rec}} \bar{\alpha}\left(\frac{1}{2}+\bar{\alpha}\right)}
$$

such that half of the negative ions flow to the wall and half recombine in the volume. For each $\beta$ value, the intersection of the $n_{\mathrm{e} 0}$ versus $\bar{\alpha}$ curves determined from (40) and (43), combined with (45), specifies the point of optimum transverse flux. Setting $\beta=1$ in (40) for maximum flux, which is consistent with the form of (43) and which we will also see from figure 8 , then together with (45), we have

$$
\frac{1}{4} v_{\mathrm{att}} n_{\mathrm{e} 0} R=\alpha_{\mathrm{s}} n_{\mathrm{e} 0} u_{\mathrm{Bi}}
$$

such that $\alpha_{\mathrm{s}}$ has the simple value

$$
\alpha_{\mathrm{s}}=\frac{v_{\mathrm{att}} R}{4 u_{\mathrm{Bi}}}
$$

Solutions for the equilibrium equations of the model are shown below for an oxygen discharge with $p=10$ mTorr, $B_{0}=300 \mathrm{G}, R L=100 \mathrm{~cm}^{2}, T_{\mathrm{e}} / T_{\mathrm{i}}=20, M_{\mathrm{i}}=32 \mathrm{amu}$, and the rate coefficients in table 1 , as used in the numerical calculations. Figure 4 gives $n_{\mathrm{e} 0} / n_{\mathrm{g}}$ versus $\bar{\alpha}$ obtained from (40) and (43), with $n_{\mathrm{g}}$ the gas density. The upper-through-lower

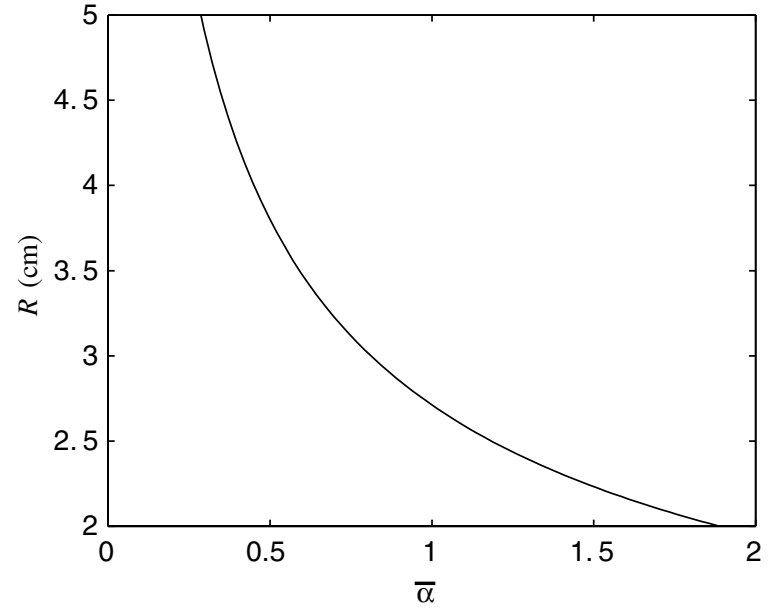

Figure 5. $R(\mathrm{~cm})$ versus $\bar{\alpha}$ for $p=10 \mathrm{mTorr}, B_{0}=300 \mathrm{G}$, $R L=100 \mathrm{~cm}^{2}$, and $T_{\mathrm{e}} / T_{\mathrm{i}}=20$.

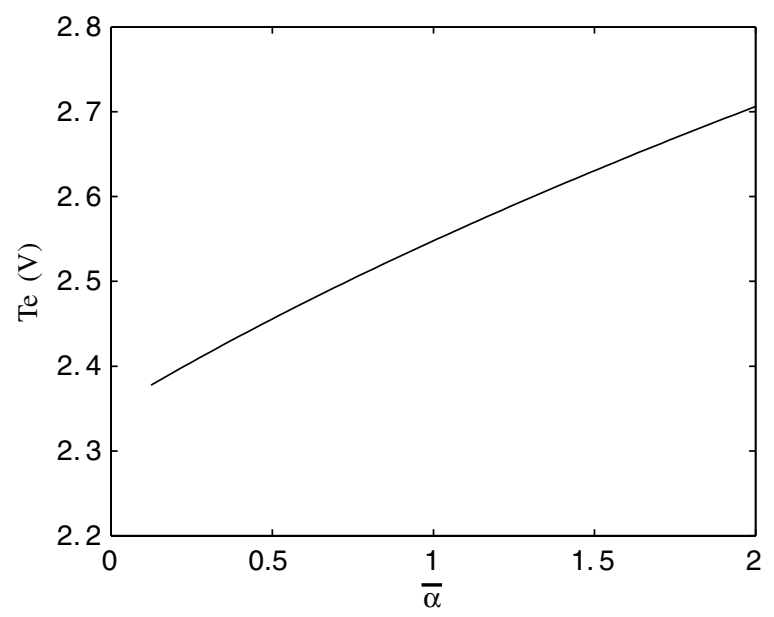

Figure 6. $T_{\mathrm{e}}$ (in volts) versus $\bar{\alpha}$ for $p=10 \mathrm{mTorr}, B_{0}=300 \mathrm{G}$, $R L=100 \mathrm{~cm}^{2}$, and $T_{\mathrm{e}} / T_{\mathrm{i}}=20$.

solid curves are for $\beta=0,0.15,0.5$ and 1 , respectively, with all physical solutions lying between the $\beta=0$ and $\beta=1$ curves. The dashed curve gives $n_{\mathrm{e} 0}$ (opt) from (45). The maximum ion flux, at the intersection of the $\beta=1$ solid curve and the dashed curve, is for $n_{\mathrm{e} 0} \approx 1.6 \times 10^{11} \mathrm{~cm}^{-3}$ and $\bar{\alpha} \approx 0.49$. Figure 5 shows the corresponding $R$ versus $\bar{\alpha}$ obtained from (36); $R \approx 3.9 \mathrm{~cm}$ at the maximum ion flux. Note from (36) and (37) that $R$ is a function of $\bar{\alpha}$, independent of $n_{\mathrm{e} 0}$; hence the loci of constant $R$ are vertical straight lines in figure 4. Figure 6 shows the corresponding $T_{\mathrm{e}}$ versus $\bar{\alpha}$ obtained from (37); $T_{\mathrm{e}} \approx 2.45 \mathrm{~V}$ at the maximum flux.

For the numerical solutions of the differential equations, the chosen variable is the central $\alpha_{0}$, not the average $\bar{\alpha}$. For comparison with the numerics, we plot the quantities versus $\alpha_{0}$. The relation between $\bar{\alpha}, \alpha_{0}$, and $\alpha_{\mathrm{s}}$, obtained in appendix $\mathrm{B}$, is

$$
\bar{\alpha}=\frac{2}{3} \alpha_{0}+\frac{1}{3} \alpha_{\mathrm{s}} .
$$

Substituting $\alpha_{\mathrm{s}}$ from (48) into (43) yields $\alpha_{0}$ as a function of $\bar{\alpha}$ for each $\beta$, which transforms figure 4 into figure 7 , which we show for $\beta=0,0.15$ and 1 . The loci of constant $R$, which 


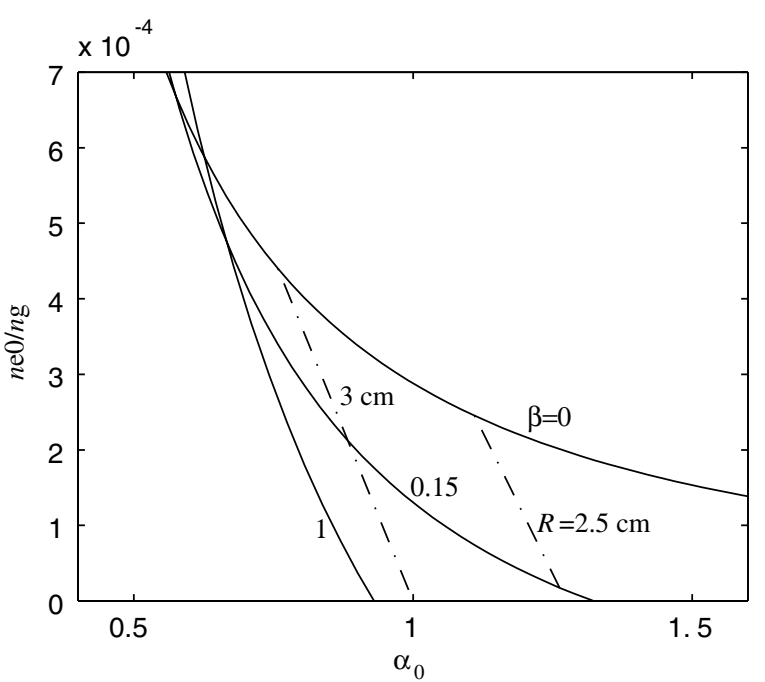

Figure 7. $n_{\mathrm{e} 0} / n_{\mathrm{g}}$ versus $\alpha_{0}$ for $p=10 \mathrm{mTorr}, B_{0}=300 \mathrm{G}$, $R L=100 \mathrm{~cm}^{2}$ and $T_{\mathrm{e}} / T_{\mathrm{i}}=20$ at $\beta=0,0.15$ and 1 (solid lines). The dot-dashed curves are the loci of constant $R$.

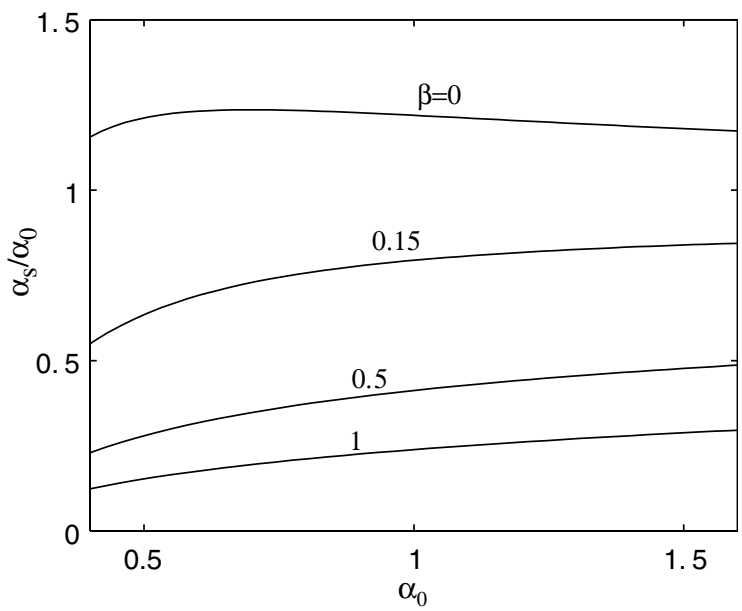

Figure 8. $\alpha_{\mathrm{s}} / \alpha_{0}$ versus $\alpha_{0}$ for $p=10 \mathrm{mTorr}, B_{0}=300 \mathrm{G}$, $R L=100 \mathrm{~cm}^{2}$ and $T_{\mathrm{e}} / T_{\mathrm{i}}=20$.

were vertical straight lines in figure 4 , are transformed to the slanted dot-dashed lines by the transformation from $\bar{\alpha}$ to $\alpha_{0}$. Figure 8 gives the ratio of edge-to-centre negative ion densities versus $\alpha_{0}$ at various values of $\beta$. We see that the negative ion density is practically flat $\left(\alpha_{\mathrm{s}} \approx \alpha_{0}\right)$ for $\beta=0$ (no edge flux), and becomes of order $\alpha_{\mathrm{s}} \approx(0.1-0.2) \alpha_{0}$ at $\beta=1$ (maximum flux). From figure 8 , we see that the product $\beta \alpha_{\mathrm{s}}$ (proportional to the edge flux) is a maximum at $\beta=1$.

\section{Comparison of simulations and model}

Some of the input and output parameters differ in the numerics and in the analytical model, but lead to a comparison of the same set of self-consistent parameters. Inputs for the numerical study are central electronegativity $\alpha_{0}$ and $n_{\mathrm{e} 0} / n_{\mathrm{g}}$. The output parameters are 'radial length' $R$, electron temperature $T_{\mathrm{e}}$ and $\beta$, the ratio of ion velocity at $x=R$ normalized to the ion Bohm velocity. $R$ appears in the expression for $v_{\mathrm{L}}$ in (7) only in the product $R L$, such that any solution is self-consistent with some value of $L$ satisfying the assumed $R L$ product. We select the values of $T_{\mathrm{e}}$ that yield the boundary conditions (21) and (22), which are also used in the analytic solutions. The numerical study explores the $n_{\mathrm{e} 0} / n_{\mathrm{g}}$ versus $\alpha_{0}$ plane to find the parameter values satisfying (21) and (22). From this solution set, we construct curves of constant $R$ and $L$, where $R$ is given by the simulation and $L$ by the fact that $R L=100 \mathrm{~cm}^{2}$, with the results shown in figure 3 . The analytical model has no ion inertia, and there are also some other approximations made; e.g. constant negative ion density in solving the electron balance equation (29) for $n_{\mathrm{e}}(x)$. Input parameters are average electronegativity $\bar{\alpha}$ and $\beta$. Output parameters $R$ and $T_{\mathrm{e}}$ depend only on $\bar{\alpha}$, and $n_{\mathrm{e} 0} / n_{\mathrm{g}}$ depends additionally on $\beta$. The transformation from $\bar{\alpha}$ to $\alpha_{0}$, described in appendix $\mathrm{B}$, also depends on $\beta$. Both the numerical and the analytical solutions in the $n_{\mathrm{e} 0} / n_{\mathrm{g}}$ versus $\alpha_{0}$ plane are delimited by two lines corresponding to $\beta=0$ and $\beta=1$. For the numerics, no solutions fulfilling the boundary conditions can be found when crossing the second $(\beta=1)$ limit, as the ions reach the Bohm velocity before the electron density and velocity go to zero, leading to a singularity. We chose the same limit for the solutions of the analytic model.

We compare figure 3 from the numerics with the analytic results in figure 7, for the corresponding limits in the $\alpha_{0}$ versus $n_{\mathrm{e} 0} / n_{\mathrm{g}}$ plane, together with curves of constant $R=3.0 \mathrm{~cm}$ with $L=33.3 \mathrm{~cm}$ and constant $R=2.5 \mathrm{~cm}$ with $L=40 \mathrm{~cm}$. Points close to the $\beta=0$ limit are easy to find numerically. There is good agreement between the analytical model and the simulations in this limit. For example, the analytical model predicts that the $\beta=0$ limit is reached at $n_{\mathrm{e} 0} / n_{\mathrm{g}}=2.75 \times 10^{-4}$ with $\alpha_{0}=1.031$, while the corresponding value for the numerics at $n_{\mathrm{e} 0} / n_{\mathrm{g}}=2.75 \times 10^{-4}$ is $\alpha_{0}=1.0$. The numerical and analytic solutions become more sensitive to errors as $\beta$ increases; as the ion velocities increase, the sensitivity increases. However, there is still a reasonable agreement of the numerics with the analytic model. In the model, with $n_{\mathrm{e} 0} / n_{\mathrm{g}}=$ $2.75 \times 10^{-4}$, the $\beta=1$ limit is reached at $\alpha_{0}=0.754$. In the numerical simulation, including the inertia terms, we find $\beta \approx 1$ at $\alpha_{0}=0.828$. Simulations were done removing the inertia terms from the numerical equations, with only small modifications to the above results. The same comparisons for constant $R$ curves also give reasonable agreement.

In the analytic model, the average $\bar{\alpha}$ is the input parameter, rather than the central $\alpha_{0}$. The curves of $n_{\mathrm{e} 0}$ versus $\bar{\alpha}$, for various values of $\beta$ do not intersect (see figure 4). However, the transformation from $\bar{\alpha}$ to $\alpha_{0}$, passing from figures 4 to 7 introduces intersection of the various $\beta$-curves, as is also found in the numerical simulations.

\section{Conclusions and further discussion}

We have considered the cross-field diffusion of a finite-length plasma with magnetized electrons and unmagnetized positive and negative ions. We have assumed that the power input is such that the electron temperature $T_{\mathrm{e}}$ is constant across the discharge, and that the ratio of length to transverse dimension is sufficiently large that the end loss can be averaged to determine a constant volume loss frequency $v_{\mathrm{L}}$. The simplest form 
of $v_{\mathrm{L}}$ involves the product $R L$, where $R$ is the transverse half-width and $L$ is the discharge length (see appendix A). We numerically examined solutions for which the electron flux and density, $\Gamma_{\mathrm{e}}$ and $n_{\mathrm{e}}$, are zero at the transverse edge, such that a pure ion-ion plasma exists there, a favourable condition for the desired ion extraction. The numerical results indicate that an approximate analytic model can be constructed, using the drift-diffusion equations, which can then be used to examine scaling and transverse ion flux optimization. To limit the parameters that need to be examined, a convenient magnetic field $B_{0}=300 \mathrm{G}$ and pressure $p=10 \mathrm{mTorr}$ are chosen, for which the electrons are magnetized and the ions (with oxygen molecular mass) are unmagnetized. The central electron densities are scaled to the neutral density. The device dimensions were roughly chosen to correspond to a current experiment, for future comparisons.

From the analytic model, solutions are easily obtained so that various parameters, fixed in the numerical solutions, can be varied. The model also gives scalings of various quantities with important parameters such as $B_{0}, p$, and rate coefficients. An 'optimum' transverse flux condition is also determined, for which half of the negative ions flow to the wall and half recombine in the volume. In the parameter space of $n_{\mathrm{e} 0}$ versus $\alpha_{0}$, the intersection of this optimum curve with the largest obtainable $\beta=1$, gives the operating condition for maximum flux.

Both numerical and analytic results indicate that at constant $R L$ and $n_{\mathrm{e} 0} / n_{\mathrm{g}}$, there is a range of solutions with decreasing $R$ and increasing $\alpha_{0}$ that vary between a value of $\beta=1$ to a value of $\beta=0$. The values of $n_{\mathrm{e} 0}$ and $\alpha_{0}$ were similar in the numerical calculations and the analytic model. To understand the role of inertia, the ion inertia effects were set to zero in some numerical calculations such that the value of $\beta=1$ could be exceeded, but at $\beta=1$ there were only small differences with the solutions including the inertia terms. At fixed values of $R L, T_{\mathrm{i}} / T_{\mathrm{e}}$, and $n_{\mathrm{e} 0} / n_{\mathrm{g}}$, the scaling of $T_{\mathrm{e}}, R$, and $\bar{\alpha}$ (or $\alpha_{0}$ ) with $B_{0}$ and $p$ can be found over the range of $\beta$-values, from the analytic model results. Some investigations of these scalings have been done numerically and have been in reasonable agreement with those predicted analytically. In particular, values of $R L=150 \mathrm{~cm}^{2}$ and $200 \mathrm{~cm}^{2}$ were also investigated, numerically and analytically, with results qualitatively as expected.

Questions concerning various assumptions that have been made in this work remain open to future investigation. The solutions that we have found do not imply the existence of a physical transverse wall at the position for which the specified electron boundary conditions are satisfied. As mentioned earlier, a pure ion-ion plasma might exist between a radius $R$ satisfying the boundary conditions and a larger radius $R_{\text {wall }}$ of a material wall. However, the stability of such solutions is not assured, and cannot be investigated within the context of our equilibrium analysis. Furthermore, if the power, and consequently $n_{\mathrm{e} 0}$, is increased above the $\beta=0$ curve with $R=R_{\text {wall }}$, we now have $\Gamma_{\mathrm{e}}(R) \neq 0$. We have not considered these solutions in this work. However, we expect, since the electric fields are initially small, that the sheath would be smaller than an electron gyroradius. In this condition electrons begin to be collected on the insulating transverse wall. Then electric fields begin to build which repel negative charges. The complicated ensuing dynamics is well worth investigating, both for the intrinsic interest of the physics and for the practical implications for ion extraction. In fact, the early operation of the experiment done in oxygen, whose approximate dimensions we have used in this analysis, may have initially operated in this regime. In the future, we plan to develop a theory appropriate to these higher values of $n_{\mathrm{e} 0} / n_{\mathrm{g}}$. The experiment will probably also be operated in a lower $n_{\mathrm{e} 0} / n_{\mathrm{g}}$ parameter range where the results can be compared with the theory developed here. An experiment more closely corresponding to a thruster would use a feedstock gas with higher electronegativity and heavier mass. The theory developed here can also be used for comparison by using a modified table of parameters values.

\section{Acknowledgments}

GL and PC acknowledge the support of ANR under contract JCJC0039. AJL and MAL acknowledge the support of California industries and University of California Discovery Grant ele07-10283 under the IMPACT programme.

\section{Appendix A. Calculation of $\nu_{\mathrm{L}}$}

Great simplicity is gained in using a one-dimensional (1D) model to describe the equilibrium of an EN plasma with the electrons magnetized by an axial field. If the device is relatively long and thin, the axial loss is in some respects small compared with the radial loss. However, because the electrons move freely in the axial direction, but are the confined species radially, the axial electron loss cannot be neglected. As seen in the main body of the paper, we look for solutions with no radial electron loss, which, without axial loss, would result in a volume balance of electrons, i.e. $K_{\text {iz }}=K_{\text {att }}$ such that $T_{\mathrm{e}}$ would be fixed at the low value appropriate to this equality, a quite singular situation. We introduce the axial loss into a 1D model, representing it as a volume loss term, using an approximation most convenient for our calculation.

Distributing the end loss $\Gamma_{z}$, at the two ends, over the volume, we have an effective volume loss term in an equivalent one-dimensional equilibrium

$$
v_{\mathrm{L}} n_{\mathrm{i}} \approx \frac{2 R}{R L} \Gamma_{z}
$$

where we assume $\Gamma_{z}$ and the axial density $n_{\mathrm{i}}$ are uniform. There are various possibilities for determining the end flux $\Gamma_{z}$. One simple approximate procedure is to use magnetically constrained transverse electrons and ambipolar axial flow, such that the ion diffusion coefficients in the transverse and axial directions are $D_{\mathrm{i}}$ and $D_{\mathrm{a}}$ respectively, where

$$
D_{\mathrm{i}}=\frac{e T_{\mathrm{i}}}{M_{\mathrm{i}} v_{\mathrm{i}}}, \quad D_{\mathrm{a}}=D_{\mathrm{i}}\left(1+\frac{T_{\mathrm{e}}}{T_{\mathrm{i}}}\right) .
$$

The simplest form for the diffusion equation in twodimensional rectangular geometry is

$$
-D_{\mathrm{i}} \frac{\partial^{2} n_{\mathrm{i}}}{\partial x^{2}}-D_{\mathrm{a}} \frac{\partial^{2} n_{\mathrm{i}}}{\partial z^{2}}=v_{\mathrm{iz}} n_{\mathrm{e}}
$$


Rescaling the $z$-variable by

$$
z=Z\left(1+T_{\mathrm{e}} / T_{\mathrm{i}}\right)^{1 / 2},
$$

we obtain

$$
-D_{\mathrm{i}}\left(\frac{\partial^{2} n_{\mathrm{i}}}{\partial x^{2}}+\frac{\partial^{2} n_{\mathrm{i}}}{\partial Z^{2}}\right)=v_{\mathrm{iz}} n_{\mathrm{e}} .
$$

Making the assumption that $n_{\mathrm{e}} \approx n_{\mathrm{e} 0}$, Kimura et al [13] have solved (A.5) to find that the diffusion scale lengths in $x$ and $Z$ are approximatively the same, and given by the shorter dimension $(R$ or $L)$, such that for $R<\left(T_{\mathrm{i}} / T_{\mathrm{e}}\right)^{1 / 2} L$, we have

$$
\frac{\mathrm{d} n_{\mathrm{i}}}{\mathrm{d} Z} \approx \frac{n_{\mathrm{i}}}{R} .
$$

Returning to the original coordinate $z$, we find

$$
\Gamma_{z} \approx D_{\mathrm{i}}\left(1+\frac{T_{\mathrm{e}}}{T_{\mathrm{i}}}\right)^{1 / 2} \frac{n_{\mathrm{i}}}{R}
$$

and substituting (A.7) into (A.1), we obtain

$$
v_{\mathrm{L}}=\frac{2 D_{\mathrm{i}}}{R L}\left(1+\frac{T_{\mathrm{e}}}{T_{\mathrm{i}}}\right)^{1 / 2} .
$$

Various approximations can be considered that bear on the accuracy of (A.5). All affect the quantitative values, but probably not the qualitative conclusions. One effect worth considering in more detail is volume recombination of positive and negative ions, which dominates the flow at higher densities. In this regime, the axial flux can be considered in a 1D approximation in $Z$, as we have previously done [14]. Using the fact that the edge negative ion flux must vanish, which can be expressed as

$$
\frac{2 D_{\mathrm{n}} \alpha_{0} n_{\mathrm{e} 0}}{l}=\frac{7}{15} K_{\mathrm{rec}} \alpha_{0}^{2} n_{\mathrm{e} 0}^{2} l
$$

we determine $l$, the edge gradient in a parabolic model. The ion flux impinging on the end-wall is then

$$
\Gamma_{\mathrm{iz}} \simeq \frac{4 D_{\mathrm{i}} \alpha_{0} n_{\mathrm{e} 0}}{l} \simeq 2\left(D_{\mathrm{i}} K_{\mathrm{rec}}\right)^{1 / 2}\left(\alpha_{0} n_{\mathrm{e} 0}\right)^{3 / 2}
$$

with the second equality obtained by substituting for $l$ from (A.9). Substituting (A.10) in (A.1), with $n_{\mathrm{i}} \simeq \alpha_{0} n_{\mathrm{e} 0}$, we obtain

$$
v_{\mathrm{L}}=\frac{2}{L}\left(\lambda_{\mathrm{i}} v_{\mathrm{ith}} K_{\mathrm{rec}} \alpha_{0} n_{\mathrm{e} 0}\right)^{1 / 2},
$$

where $D_{\mathrm{i}} \simeq \lambda_{\mathrm{i}} v_{\text {ith }}$, with $\lambda_{\mathrm{i}}$ and $v_{\text {ith }}$ the ion-neutral mean free path and ion thermal velocity. By comparing (A.11) with (A.8), we can find a transition with increasing $n_{\mathrm{i}}$ beyond which the end loss gradient is increasingly determined by volume recombination. This transition occurs when

$$
4 R^{2} K_{\text {rec }} \frac{n_{\mathrm{i}}}{D_{\mathrm{i}}}=\frac{T_{\mathrm{e}}}{T_{\mathrm{i}}} .
$$

From (A.11) we see that $v_{\mathrm{L}}$ no longer has the scaling in (A.8). However, for a typical set of parameters, (A.11) and (A.8) gave similar values of $v_{\mathrm{L}}$. We use $v_{\mathrm{L}}$ from (A.8) in the calculations.

\section{Appendix B. Relations among $\bar{\alpha}, \alpha_{0}$ and $\alpha_{\mathrm{s}}$}

To determine the ambipolar electric field $E$, the drift-diffusion relations for the positive ion, negative ion and transverse electron fluxes are used, along with the ambipolar flux condition

$$
\Gamma_{\mathrm{i}}=\Gamma_{\mathrm{n}}+\Gamma_{\perp \mathrm{e}}
$$

Assuming quasi-neutrality, $n_{\mathrm{i}}=n_{\mathrm{n}}+n_{\mathrm{e}}$, this yields

$$
\begin{aligned}
& -D_{\mathrm{i}} \frac{\mathrm{d} n_{\mathrm{n}}}{\mathrm{d} x}-D_{\mathrm{i}} \frac{\mathrm{d} n_{\mathrm{e}}}{\mathrm{d} x}+\mu_{\mathrm{i}}\left(n_{\mathrm{n}}+n_{\mathrm{e}}\right) E \\
& \quad=-D_{\mathrm{i}} \mathrm{d} n_{\mathrm{n}} \mathrm{d} x-\mu_{\mathrm{i}} n_{\mathrm{n}} E-D_{\perp \mathrm{e}} \frac{\mathrm{d} n_{\mathrm{e}}}{\mathrm{d} x}-\mu_{\perp \mathrm{e}} n_{\mathrm{e}} E .
\end{aligned}
$$

Solving for the field, we obtain

$$
E=\frac{D_{\mathrm{i}}-D_{\perp \mathrm{e}}}{2 \mu_{\mathrm{i}} n_{\mathrm{n}}+\left(\mu_{\mathrm{i}}+\mu_{\perp \mathrm{e}}\right) n_{\mathrm{e}}} \frac{\mathrm{d} n_{\mathrm{e}}}{\mathrm{d} x} .
$$

The negative ion flux is then

$$
\Gamma_{\mathrm{n}}=-D_{\mathrm{i}} \frac{\mathrm{d} n_{\mathrm{n}}}{\mathrm{d} x}-\frac{\mu_{\mathrm{i}} n_{\mathrm{n}}\left(D_{\mathrm{i}}-D_{\perp \mathrm{e}}\right)}{2 \mu_{\mathrm{i}} n_{\mathrm{n}}+\left(\mu_{\mathrm{i}}+\mu_{\perp \mathrm{e}}\right) n_{\mathrm{e}}} \frac{\mathrm{d} n_{\mathrm{e}}}{\mathrm{d} x} .
$$

Equating (B.4) to (39) with $n_{\mathrm{n}}=\bar{n}_{\mathrm{n}}$ on the right hand side, and with $n_{\mathrm{e}}$ given by (31), we obtain

$$
\begin{aligned}
-D_{\mathrm{i}} & \frac{\mathrm{d} n_{\mathrm{n}}}{\mathrm{d} x}=-\frac{1}{2} n_{\mathrm{e} 0} \frac{D_{\mathrm{i}}-D_{\perp \mathrm{e}}}{2+\left(1+\mu_{\perp \mathrm{e}} / \mu_{\mathrm{i}}\right)[1+\cos (\pi x / R)](2 \bar{\alpha})^{-1}} \\
& \times \frac{\pi}{R} \sin \left(\frac{\pi x}{R}\right)+\frac{1}{2} n_{\mathrm{e} 0}\left[\nu_{\mathrm{att}}-K_{\mathrm{rec}} n_{\mathrm{e} 0}\left(\bar{\alpha}+2 \bar{\alpha}^{2}\right)\right] x \\
+ & \frac{1}{2} n_{\mathrm{e} 0}\left(\nu_{\mathrm{att}}-K_{\mathrm{rec}} n_{\mathrm{e} 0} \bar{\alpha}\right) \frac{R}{\pi} \sin \left(\frac{\pi x}{R}\right) .
\end{aligned}
$$

Integrating this equation from $x=0$ to $x=R$, we find

$$
\begin{aligned}
& -D_{\mathrm{i}}\left(\alpha_{\mathrm{s}}-\alpha_{0}\right)=-\frac{D_{\mathrm{i}}-D_{\perp \mathrm{e}}}{1+\mu_{\perp \mathrm{e}} / \mu_{\mathrm{i}}} \bar{\alpha} \ln \left[1+\frac{1+\mu_{\perp \mathrm{e}} / \mu_{\mathrm{i}}}{2 \bar{\alpha}}\right] \\
& +\left[v_{\mathrm{att}}-K_{\mathrm{rec}} n_{\mathrm{e} 0}\left(\bar{\alpha}+2 \bar{\alpha}^{2}\right)\right] \frac{R^{2}}{4}+\left[v_{\mathrm{att}}-K_{\mathrm{rec}} n_{\mathrm{e} 0} \bar{\alpha}\right] \frac{R^{2}}{\pi^{2}} .
\end{aligned}
$$

To relate $\bar{\alpha}$ to $\alpha_{0}$ and $\alpha_{\mathrm{s}}$, we assume a parabolic negative ion density profile, consistent with the first three terms in a power series expansion in $x$ of the solution of (B.5)

$$
\bar{\alpha}=\frac{2}{3} \alpha_{0}+\frac{1}{3} \alpha_{\mathrm{s}}
$$

Solving (B.7) for $\alpha_{0}$ and inserting this into (B.6), we obtain the relation between $\bar{\alpha}$ and $\alpha_{\mathrm{s}}$ given in (43).

\section{References}

[1] Meige A, Leray G, Raimbault J-L and Chabert P 2008 Appl. Phys. Lett. 92061501

[2] Stamate E and Ohe K 1998 J. Appl. Phys. 842450

[3] Kanakasabapathy S K, Overzet L J, Midha V and Economou D J 2001 Appl. Phys. Lett. 7822

[4] Economou D J 2007 Appl. Surf. Sci. 2536672

[5] Chabert P 2007 Patent Number WO 2007/065915 A1 
[6] Aanesland A, Leray G and Chabert P 2008 44th AIAA/ASME/SAE/ASEE Joint Propulsion Conf. and Exhibit (Hartford, CT, 21-23 July) AIAA-2008-5198

[7] Kawai R and Mieno T 1997 Japan. J. Appl. Phys. 36 L1123

[8] Chabert P, Sheridan T E, Boswell R W and Perrin J 1999 Plasma Sources Sci. Technol. 8561

[9] Walton S G, Leonhardt D, Fernsler R F and Meger R A 2002 Appl. Phys. Lett. 81 987-9

[10] Corr C S, Plihon N and Chabert P 2006 J. Appl. Phys. 99103302
[11] Franklin R N and Snell J 1999 J. Phys. D: Appl. Phys. 321031

[12] Lieberman M A and Lichtenberg A J 2005 Principles of Plasma Discharges and Materials Processing 2nd edn (New York: Wiley-Interscience)

[13] Kimura T, Lichtenberg A J and Lieberman M A 2001 Plasma Sources Sci. Technol. 10430

[14] Lichtenberg A J, Kouznetsov I G, Lee Y T, Lieberman M A, Kaganovich I D and Tsendin L D 1997 Plasma Sources Sci. Technol. 6437 